Pontifícia U Miversidade $C_{\text {atólica }}$

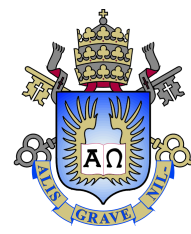

Victor Lopes da Silva

Propriedades magnéticas, de transporte e emergentes em sistemas nanoscópicos fortemente correlacionados

Tese de Doutorado

Tese apresentada como requisito parcial para obtenção do grau de Doutor pelo Programa de Pós-graduação em Física da PUCRio.

Orientador: Prof. Enrique Victoriano Anda 


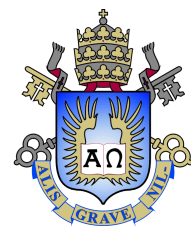

Victor Lopes da Silva

\section{Propriedades magnéticas, de transporte e emergentes em sistemas nanoscópicos fortemente correlacionados}

Tese apresentada como requisito parcial para obtenção do grau de Doutor pelo Programa de Pós-graduação em Física da PUCRio. Aprovada pela Comissão Examinadora abaixo assinada.

Prof. Enrique Victoriano Anda

Orientador

Departamento de Física PUC-Rio

Prof. George Balster Martins

UFF

Prof. Caio Henrique Lewenkopf

UFF

Prof. Edson Vernek

UFU

Prof. Mucio Amado Continentino

CBPF

Prof. Márcio da Silveira Carvalho

Coordenador Setorial do Centro Técnico Científico PUC-Rio 
Todos os direitos reservados. É proibida a reprodução total ou parcial do trabalho sem autorização da universidade, do autor e do orientador.

\section{Victor Lopes da Silva}

Graduou-se em Física pela Universidade Federal do Rio de Janeiro (UFRJ). Fez mestrado no Departamento de Física da Pontifícia Universidade Católica do Rio de Janeiro (PUCRio).

Ficha Catalográfica

Lopes da Silva, Victor

Propriedades magnéticas, de transporte e emergentes em sistemas nanoscópicos fortemente correlacionados / Victor Lopes da Silva; orientador: Enrique Victoriano Anda. - Rio de janeiro: PUC-Rio, Departamento de Física, 2018.

v., 132 f: il. color. ; $30 \mathrm{~cm}$

Tese (doutorado) - Pontifícia Universidade Católica do Rio de Janeiro, Departamento de Física.

Inclui bibliografia

1. Física - Teses. 2. Efeito Kondo;. 3. Interação spinórbita;. 4. Quebra de simetria;. 5. Sistemas nanoscópicos;. 6. Spintrônica;. 7. Hamiltoniano de Anderson;. 8. Pontos Quânticos;. I. Victoriano Anda, Enrique. II. Pontifícia Universidade Católica do Rio de Janeiro. Departamento de Física. III. Título. 


\section{Agradecimentos}

Agradeço primeiramente aos meus pais José e Luciene, ao meu irmão Felipe e a todos os familiares pelo seu apoio incondicional.

Ao professor Enrique Victoriano Anda por aceitar me orientar tanto no mestrado quanto no doutorado, guiando-me no mundo acadêmico, bem como pelo enriquecimento intelectual e moral nestes anos.

Aos colaboradores Pablo Roura-Bas e Ignacio J. Hamad pela atenção que me foi dada durante as visitas e permanência na PUC-Rio e a ajuda com a programação.

Aos colaboradores George B. Martins e Ronald Padilla, com quem desenvolvi parte do trabalho desta tese.

À dedicação apresentada por Marco Manya nas pesquisas realizadas.

Aos colegas de pós-graduação e professores pelo excelente ambiente de trabalho.

Aos funcionários do Departamento de Física Julinho, Márcia, Giza e Juliana, fundamentais para o bom funcionamento da atividade acadêmica.

Ao amigo Amintor Dusko pelas boas conversas que ajudaram nos momentos mais árduos.

Às agências de fomento CAPES e CNPq pela bolsa de doutorado.

À PUC-Rio por disponibilizar sua infraestrutura.

À todos que de alguma forma contribuíram para o desenvolvimento desta tese. 


\section{Resumo}

Lopes da Silva, Victor; Victoriano Anda, Enrique. Propriedades magnéticas, de transporte e emergentes em sistemas nanoscópicos fortemente correlacionados. Rio de Janeiro, 2018. 132p. Tese de Doutorado - Departamento de Física, Pontifícia Universidade Católica do Rio de Janeiro.

Esta tese investiga as propriedades eletrônicas de sistemas nanoscópicos com interações de muitos corpos, dando origem ao efeito Kondo.

Primeiramente estudamos a transição $\mathrm{SU}(4)-\mathrm{SU}(2)$ devido a um campo magnético externo e as propriedades de filtro de spin de um nanossistema de dois pontos quânticos capacitivamente acoplados. A transição é caracterizada pela diferença entre as polarizações de spin da ocupação eletrônica nos dois pontos quânticos, como uma função do potencial de porta aplicado sobre os pontos quânticos. Apesar do fato de que o campo magnético externo quebra a simetria $\mathrm{SU}(4)$ do Hamiltoniano, o estado fundamental a preserva, como uma propriedade emergente, na região do espaço de parâmetros onde os elétrons não estão polarizados. As propriedades de filtro de spin devido à população eletrônica spin polarizada nos pontos quânticos também é discutida. Estas propriedades são estudadas usando o formalismo dos operadores de projeção, que descreve de forma muito acurada a física associada ao estado fundamental dos sistemas Kondo.

No capítulo subsequente, analisamos os efeitos da interação spin-órbita num ponto quântico conectado a contatos, representados pelo modelo da impureza de Anderson no efeito Kondo. Contrariamente ao resultado prévio de vários outros autores, nós mostramos que a interação spin-órbita reduz exponencialmente a temperatura Kondo enquanto a ação da interação no próprio ponto quântico pode ser um mecanismo de destruição do regime Kondo, conforme quebra a simetria $\mathrm{SU}(2)$.

Usando o modelo de Anderson com acoplamento spin-órbita nós propomos um transistor de spin feito de um ponto quântico conectado a uma nanofaixa submetida à interação spin-órbita Rashba, depositada sobre um substrato ferromagnético. O ponto quântico também é conectado a dois contatos metálicos laterais, através do qual a corrente flui ao longo do sistema. A interação spin-órbita Rashba cria um mecanismo de inversão do spin no ponto quântico. Nós mostramos que o sistema é capaz de operar como um transistor de spin.

\section{Palavras-chave}

Efeito Kondo; Interação spin-órbita; Quebra de simetria; Sistemas nanoscópicos; Spintrônica; Hamiltoniano de Anderson; Pontos Quânti$\cos$; 


\section{Abstract}

Lopes da Silva, Victor; Victoriano Anda, Enrique (Advisor). Magnetic, transport and emergent properties in nanoscopic and strongly correlated systems. Rio de Janeiro, 2018. 132p. Tese de doutorado - Departamento de Física, Pontifícia Universidade Católica do Rio de Janeiro.

This thesis investigates the electronic properties of nanoscopic systems under the presence of many body interactions, given rise to the Kondo effect. Firstly we studied the SU(4)-SU(2) crossover driven by an external magnetic field and the spin-filter properties of a capacitively coupled double quantum dot nanosystem. The crossover is characterized by the difference between the spin polarization of the electronic occupation at the double quantum dot, as a function of the gate potential applied to the quantum dots. Despite the fact that the external magnetic field breaks the SU(4) symmetry of the Hamiltonian, the ground state preserves it, as an emergent property, in a region in the parameter space where the electron are not polarized. The spinfilter properties due the spin polarized electronic population at the dots is also discussed. These properties are studied using the projector projection operator approach, which describes very accurately the physics associated to the ground state of Kondo systems.

In a subsequent chapter, we analyze the effect of the spin-orbit interaction in a quantum dot connected to leads, represented by the Anderson impurity model on the Kondo effect. Contrary to several other authors previous results, we show that the Rashba spin-orbit interaction exponentially reduces the Kondo temperature while the action of the interaction on the quantum dot itself could be a mechanism of destroying the Kondo regime, as it breaks $\mathrm{SU}(2)$ symmetry.

Using the Anderson model with spin-orbit coupling we propose a spin transistor device made of a quantum dot connected to a Rashba spinorbit interacting nanoribbon, deposited on a ferromagnetic substrate. The quantum dot is also connected to two lateral metallic contacts, through which the current flows along the system. The Rashba spin-orbit interaction creates a spin-flip mechanism at the quantum dot. We show that the system is capable of operating as a spin-transistor.

\section{Keywords}

Kondo Effect; Spin-orbit interaction; Broken symmetry; Nanoscopic systems; Spintronics; Anderson Hamiltonian; Quantum Dot; 


\section{Sumário}

1 Introdução $\quad 14$

1.1 Propriedades de transporte em função da temperatura 14

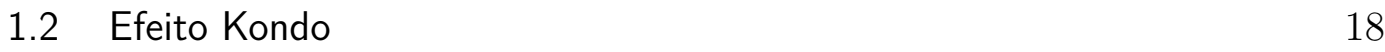

1.3 Pontos quânticos 22

1.4 Modelo da Impureza de Anderson 25

$\begin{array}{lll}1.5 & \text { Objetivos e sistemas analisados } & 30\end{array}$

2 Transição SU(4)-SU(2) e propriedades de filtro de spin de um nanossistema de dois pontos quânticos $\quad 32$

2.1 Introdução 32

2.2 Descrição do sistema 35

2.3 Tratamento do sistema com duas impurezas pelo formalismo de operadores de projeção 36

2.4 Transição SU(4)-SU(2) 38

2.5 Filtro de Spin 46

2.6 Conclusões 50

3 Hamiltoniano de Anderson com interação spin-órbita 51

3.1 Introdução 51

3.2 Interação spin-órbita Rashba $\quad 53$

3.3 Interação spin-órbita Dresselhaus $\quad 54$

3.4 Interação spin-órbita no volume 55

3.5 Influência da interação spin-órbita sobre o efeito Kondo 59

4 Corrente de spin polarizada em um ponto quântico conectado a um mar de Fermi com interação spin-órbita $\quad \mathbf{6 3}$

4.1 Introdução 63

4.2 Descrição do sistema $\quad 65$

$\begin{array}{lll}4.3 \text { Teoria } & 67\end{array}$

$\begin{array}{lll}4.4 & \text { Resultados e discussões } & 71\end{array}$

4.4.1 Contatos ferromagnéticos e não magnéticos 74

4.4.2 Contatos ferromagnéticos antiparalelos 81

4.5 Conclusões e perspectivas futuras 82

5 Conclusões e perspectivas futuras $\quad \mathbf{8 4}$

5.1 Introdução 84

5.2 Sistema de dois pontos quânticos acoplados capacitivamente 84

5.3 Interação spin-órbita $\quad 85$

5.4 Transistor de spin $\quad 86$

5.5 Sistemas fortemente dependentes da frequência 87

5.6 Estudo da repulsão Coulombiana no volume 88

5.6.1 Descrição do sistema e do formalismo utilizado 88

5.6.2 Extensão do formalismo do DMFT ao espaço das energias 88

5.6.3 Sistema de dois sítios e dois elétrons 89 
5.6.4 Sistema de três sítios e três elétrons 93

$\begin{array}{lr}\text { Referências bibliográficas } & 97\end{array}$

A Formalismo de Operadores de Projeção 109

A.1 Descrição do método 109

A.2 Aplicação do formalismo de operadores de projeção ao Hamiltoniano de Anderson 111

A.3 Equivalência do formalismo usando funções de Green 116

A.4 Importância dos termos não diagonais 119

A.5 Extensão do formalismo de operadores de projeção para sistemas com duas impurezas 121

$\begin{array}{lll}\text { A.6 Resumo do apêndice } & 124\end{array}$

B Cálculo da condutância $\quad 125$

C Transformação do Hamiltoniano com interação spin-órbita 130 


\section{Lista de figuras}

Figura 1.1 Comportamento da resistividade do sódio para diferentes valores da temperatura. Observe a saturação da resistividade a partir dos $10 \mathrm{~K}$, saturação denominada resistividade residual, proveniente dos defeitos na rede cristalina e da interação elétronfônon [1].

Figura 1.2 Resistência $(\Omega)$ como função da temperatura $(K)$ para o mercúrio, mostrando a transição para o estado supercondutor em $4.20 \mathrm{~K}$. A resistência reduz de cerca de $0.1 \Omega$ a $10^{-5} \Omega$ ao alterar a temperatura em $0.01 \mathrm{~K}$, atingindo, posteriormente, valores imensuráveis $[2,3]$.

Figura 1.3 Resistência elétrica como função da temperatura para uma liga de cobre e ferro em diferentes concentrações. Note a pequena concentração de ferro, caracterizando-o como uma impureza. As barras indicam a localização do mínimo na resistência, [4]

Figura 1.4 Mínimo na resistividade para ligas de molibdênio-nióbio com $1 \%$ de ferro $[5,6]$.

Figura 1.5 Comparação da expressão da resistividade obtida por Kondo com os resultados experimentais. Impurezas de ferro diluídas em uma matriz de ouro [7, 8].

Figura 1.6 Condutância através do PQ como função do potencial de porta, a temperatura de $10 \mathrm{mK}$ [9]. Da esquerda para a direita, o primeiro pico corresponde a voltagem em que um elétron entra no PQ. E a cada novo pico subsequente, um novo elétron entra no $\mathrm{PQ}$.

Figura 1.7 Imagem de um PQ obtida a partir do escaneamento de microscopia eletrônica [10].

Figura 1.8 Condutância diferencial. Observe nos painéis da esquerda que a medida que a temperatura aumenta de $90 \mathrm{mK}$ a $600 \mathrm{mK}$ o pico na condutância diminui até sumir. Nos painéis da direita observamos a separação dos picos com o aumento do campo para $90 \mathrm{mK}$, assinatura do Efeito Kondo [10].

Figura 1.9 Funções de onda do cério obtidas pelo cálculo de densidade funcional para a configuração $4 \mathrm{f}^{1} 5 \mathrm{~d}^{1} 6 \mathrm{~s}^{2}$ [7]. As setas no canto superior direito indicam o tamanho do raio da célula de Wigner-Seitz para $\alpha$ e $\gamma$-Ce. Observe como o estado $4 \mathrm{f}$ é mais localizado que a célula de Wigner-Seitz e que os outros estados.

Figura 1.10 Densidade espectral, $\pi \Delta \rho(\omega)$, para o modelo de Anderson simétrico com $U / \pi \Delta=2.5$ e ocupação $\left\langle n_{0}\right\rangle=1$ [11]. Quando o sistema está no regime Kondo, o sistema apresenta, além dos picos em $\epsilon_{0}$ e $\epsilon_{0}+U$, o pico no nível de Fermi. A largura do pico a meia altura é similar ao valor da $T_{K}$. 
Figura 2.1 Sistema de dois PQs capacitivamente acoplados na presença de um campo magnético externo, que age somente sobre os PQs. $U^{\prime}(U)$ é a repulsão Coulombiana interponto (intraponto) os quais estão conectados a dois contatos por elementos de matriz $V$, não mostrados na figura. O sistema apresenta duas geometrias, na primeira com o PQ embebido nos contatos (a) e na segunda conectado lateralmente (b). Como será mostrado na seção 2.5, um pequeno campo magnético pode polarizar o spin da corrente transmitida através dos PQs, com polarização paralela ao campo para os PQs embebidos (a) e antiparalela para o caso de conexão lateral (b), como ilustrado esquematicamente.

Figura 2.2 A função $f_{1}(\xi)$ resolvida autoconsistentemente para diferentes valores de $\epsilon_{0}$ com um campo magnético externo nulo. As soluções para a correção da energia do estado fundamental, $\Delta E$, obtidas pelos POA são indicadas pelas setas. Todas as grandezas estão expressas em unidades de $\Delta$.

Figura 2.3 Números de ocupação eletrônica nos PQs $\left\langle n_{\sigma}\right\rangle$ e $\left\langle n_{t}\right\rangle=$ $\left\langle n_{\uparrow}\right\rangle+\left\langle n_{\downarrow}\right\rangle$ como função do potencial de porta $\epsilon_{0}$ [12]. (a) comparação dos resultados de $\left\langle n_{\uparrow}\right\rangle,\left\langle n_{\downarrow}\right\rangle$, e $\left\langle n_{t}\right\rangle$ obtidos com o POA (símbolos) e MFSBA (curvas sólidas), para $B=3.2 \times 10^{-3} \Delta$. Observe que os resultados para POA e bósons escravos concordam semiquatitativamente. Também no mesmo painel a ocupação na ausência de campo magnético externo. (b) resultados dos POA $\left\langle n_{\sigma}\right\rangle$ para quatro valores de campo magnético externo. Todos os resultados em ambos os painéis são para $D=64.0 \Delta \mathrm{e}$ são apresentados em unidades de $\Delta=1$.

Figura 2.4 Resultados do formalismo de MFSBA, em unidades de $\Delta=1$, para $\left\langle n_{\uparrow}\right\rangle$ (linha contínua vermelha), $\left\langle n_{\downarrow}\right\rangle$ (curva azul tracejada), and $\left\langle n_{t}\right\rangle$ (curva verde pontilhada), como função do potencial de porta $\epsilon_{0}$ em um sistema de dois PQs acoplados capacitivamente, para $D=64.0 \Delta, U \rightarrow \infty, U^{\prime}=64.0 \Delta$ e $B=$ $3.2 \times 10^{-3} \Delta[12]$. Observe que os resultados são qualitativamente os mesmos que o obtido para $U=U^{\prime} \rightarrow \infty$, veja a figura 2.3.

Figura 2.5 Gráfico semilog de $B_{\max }=f\left(\epsilon_{0}^{\max }\right)$ obtido usando POA para três valores diferentes de semilargura de banda $D$. Os resultados estão apresentados em unidades de $\Delta=1$. No gráfico maior temos a comparação do resultado com $D=64 \Delta$ com as curvas para as diferentes simetrias da temperatura Kondo, $\mathrm{SU}(4)$ na curva pontilhada e $\mathrm{SU}(2)$ na tracejada, que foram obtidas a partir da equação (2-8). Este resultado mostra que $B_{\max }$ tem uma escala de energia intermediária entre as $T_{K}^{S U(4)}$ e $T_{K}^{S U(2)}$. Apresentamos no gráfico menor inserido os resultados para $D=44.4 \Delta$ e $D=16 \Delta$, além do resultado com $D=64 \Delta$. Nele verificamos a ausência de $D$ na dependência exponencial do fator $a$ (ver equação (2-7)), que configura o caráter universal da transição SU(4)-SU(2) comandada pelo campo magnético. 
Figura 2.6 Resultados do POA para $\left\langle n_{\uparrow}\right\rangle$ (linhas sólidas) e $\left\langle n_{\downarrow}\right\rangle$ (linhas tracejadas), como função do desdobramento Zeeman $B$ para quatro valores de $\epsilon_{0}=-9.6 \Delta$ (círculos vermelhos), $-11.1 \Delta$ (triângulos invertidos magenta), $-14.4 \Delta$ (quadrados verdes) e $-19.2 \Delta$ (triângulos azuis). Note a polarização gradual do sistema quando no regime de flutuação de carga (círculos vermelhos) e a mudança abrupta, a campos pequenos, do Kondo $\mathrm{SU}(4)$ para $\mathrm{SU}(2)$ quando o sistema está dentro da região Kondo SU(4) a campo nulo (triângulos azuis). Todos os resultados apresentados em unidades de $\Delta=1$ e realizados para $D=$ $64.0 \Delta$, exceto para $\epsilon_{0}=-11.1 \Delta$, que foi obtido com $D=44.4 \Delta .44$

Figura 2.7 Mesmos dados da figura 2.6, mas agora traçados como função de $B / T_{K}^{S U(4)}$ ao invés de $B$.

Figura 2.8 No eixo vertical da esquerda, $\left\langle n_{\sigma}\right\rangle$ como função de $B$ para $\epsilon_{0}=-11.11 \Delta$ (triângulos magenta), já no eixo vertical da direita, em escala logarítmica, as temperaturas Kondo $T_{K}^{S U(4)}$ (linha pontilhada) e $T_{K}^{S U(2)}$ (linha tracejada) com $B=0$ são representadas por linhas horizontais. A linha sólida negra é a temperatura Kondo para um "estado de transição", como função de $B$, obtido pelo método variacional da interpolação entre estados $\mathrm{SU}(4)$ e $\mathrm{SU}(2)$ [13, 14]. Resultados apresentados em unidades de energia com $\Delta=1$ para $\epsilon_{0}=-11.1 \Delta$ e $D=44.4 \Delta$.

Figura 2.9 Condutância para cada spin, $C^{\sigma}$, como função do potencial de porta $\epsilon_{0}$, obtida por ambos os formalismos. Os resultados do POA são traçados como símbolos, já o do MFSBA como linhas. No painel (a) para o caso da geometria do PQ embebido e no (b) para o conectado lateralmente. Os parâmetros usados para cálculo são $D=64.0 \Delta, B=3.2 \times 10^{-3} \Delta$ e $U=U^{\prime} \rightarrow \infty$, em unidades de $\Delta=1$.

Figura 3.1 Elétrons são injetados ao longo da direção $\boldsymbol{x}$ em uma região onde um campo elétrico atua na direção $z$. Quando o spin dos elétrons injetados não estão alinhados na direção da interação SO Rashba, o spin eletrônico sofre uma precessão como ilustrado no painel inferior.

Figura 3.2 Cadeia linear infinita com interação spin-órbita.

Figura 3.3 Relação de dispersão $\epsilon_{k \nu}$ para cada helicidade $\nu$, equação (3-22). Resultados em unidades de $t$ e com $|\gamma|=0.5$.

Figura 3.4 Gráfico da densidade de estados $\rho_{\nu}(\omega)$ de uma cadeia linear com interação SO. Mesmos parâmetros da figura 3.3 e em unidades de $t$.

Figura 3.5 Gráfico da relação de dispersão efetiva que cada combinação simétrica ou antissimétrica se acopla. Resultados em unidades de $t$ e com os mesmos parâmetros das figura 3.3. 
Figura 4.1 (a) Diagrama do PQ (círculos vazios) acoplado a um condutor unidimensional infinito com interação SO no espaço e na representação de spin (círculos azuis preenchidos). Esta estrutura em (b) é representada por dois círculos vazios com uma interação efetiva, $\tilde{t}$, entre eles. Em (c) nós representamos a estrutura em (b) conectada aos contatos -1 e 1, onde a corrente flui. O spin eletrônico é indicado em cada canal e as setas horizontais representam a corrente eletrônica.

Figura 4.2 O painel (a) mostra a relação de dispersão para $\alpha=0$ e $\alpha=0.4$, ambos com $\delta=0$. No painel (b) a vizinhança de $k=0$ para um valor fixo de $\alpha=0.4$ e vários valores de $\delta$. As valores estão expressos em unidades de $t$.

Figura 4.3 Comportamento de $\tilde{t}(\omega)$ para $V=0.3, \alpha=0.4 \mathrm{e}$ $\delta=0.05$. A parte real está graficada como linha sólida (em vermelho) e a imaginária com a tracejada (em azul). No gráfico menor são apresentados os mesmos resultados, mas para uma escala de $\omega$ maior.

Figura 4.4 (a) Condutância no contato $i=1$ para cada spin, $C^{\sigma}$, e (b) polarização da corrente, $p$, como função do potencial de porta $\epsilon_{0}$, ambos os painéis sem repulsão Coulombiana. No painel (a) para cada spin $\sigma$ e dois valores do acoplamento SO Rashba, $\alpha=0.1$ e $\alpha=0.4$, enquanto no painel (b) para cinco valores de $\alpha, \alpha=0.05,0.1,0.2,0.3$ e 0.4 .

Figura 4.5 Ocupação no PQ, $\left\langle n_{\sigma}\right\rangle$, como função de $\epsilon_{0}$, para $\alpha=0.4$ e vários valores de $U$.

Figura 4.6 Condutância $C^{\sigma}$ para o contato $i=1$ como função do potencial de porta $\epsilon_{0}$, para $\alpha=0.1$ e $\alpha=0.4$.

Figura 4.7 Parte imaginária da função de Green diagonal sem repulsão Coulombiana para $\alpha=0.1$ e $\alpha=0.4$. Os valores do potencial de porta e do nível de Fermi são mantidos fixos em $\epsilon_{0}=-0.5$ e $\epsilon_{F}=-1.95$.

Figura 4.8 (a) Polarização da corrente, $p$, como função do potencial de porta $\epsilon_{0}$ para diferentes $\alpha$, com $U=0.3$, e (b) para $\alpha=0.4$ e três valores de $U$.

Figura 4.9 Condutância spin para baixo, $C^{\downarrow}$, no contato $i=1$ como função do potencial de porta, $\epsilon_{0}$ para dois valores de $\alpha$. Painel (a) com repulsão Coulombiana $U=0$ e painel (b) com $U=0.3$.

Figura 5.1 Susceptibilidade magnética da impureza $\chi_{i m p}$ como função do elemento diagonal da impureza $\epsilon_{0}$. O nível de Fermi está localizado em $\epsilon_{F}=-1.9$ e $V=0.25$. Resultados em unidades de $t$.

Figura 5.2 Diagrama representando a equivalência do sistema de muitos corpos, na esquerda, com o sistema de um corpo, na direita. Os sítios hachurados possuem repulsão Coulombiana local entre os elétrons no sítio através das renormalizações. 
Figura 5.3 O gráfico apresenta a solução da energia do estado fundamental usando diferentes formalismos, o DMFT (círculos e linha contínua vermelha), a solução analítica e exata do sistema (linhas azul tracejada), obtida pelos autovalores do Hamiltoniano e pelo POA junto a filosofia do DMFT no espaço das energias. O eixo y está normalizado pelo valor da energia quando $U=0$.

Figura 5.4 No lado esquerdo temos o sistema de muitos corpos e no direito o sistema de um corpo.

Figura 5.5 No lado esquerdo, o sistema antes da combinação simétrica antisimétrica e, no direito, o sistema após a combinação.

Figura 5.6 Energia do estado fundamental para o sistema de três sítios e três elétrons. Apresentamos a solução da filosofia do DMFT no espaço das energias e POA (linha contínua vermelha), equação (5-30), a solução analítica exata (linha tracejada verde), equação (5-26), e a solução numérica dos autovalores da matriz na equação (5-24) (quadrados azuis). O eixo y está normalizado pelo valor da energia quando $U=0$.

Figura A.1 Representação diagramática do subespaço $S_{1}$, composto por um único estado com o do mar de Fermi cheio e a impureza e a banda de condução vazias.

Figura A.2 Construção do subespaço $S_{2}$, representando esquematicamente três famílias de estados construídas com sucessivas aplicações do operador $H_{21}$.

Figura A.3 Analogia dos sítios de uma rede de Bethe com os estados construídos com o formalismo de operadores de projeção.

Figura A.4 Diagrama representando uma contribuição não diagonal. Os sítios destacados são renormalizados por termos diagonais.

Figura A.5 Representação diagramática de escolha do subespaço $S_{1}$ para o sistema com duas impurezas. O estado escolhido para constituir este subespaço possui as duas impurezas vazias e os mares de Fermi no estado fundamental.

Figura A.6 Diagrama apresentando a construção do subespaço $S_{2}$, com a mesma notação da figura A.5. Na parte superior da figura, temos a única família de estados que constitui o subespaço $S_{1}$. As setas abaixo deste estado mostram a passagem do subespaço $S_{1}$ a primeira família de estados do subespaço $S_{2}$, estados I, com a impureza ocupada e um buraco no mar de Fermi correspondente a esta impureza. Também ilustramos a passagem aos estados II, onde o elétron da impureza dirige-se à banda de condução.

Figura B.1 Representação trigonométrica da função de Green $G_{00}^{\sigma \sigma}(\omega)$, equação (B-8). 


\section{Introdução}

\section{1}

\section{Propriedades de transporte em função da temperatura}

Nesta tese temos por objetivo investigar as propriedades eletrônicas e, particularmente, as de transporte de sistemas nanoscópicos que possuam uma região fortemente correlacionada, como um ponto quântico (PQ) ou uma estrutura de PQ's.

Estamos considerando a condutância elétrica destes sistemas como uma propriedade central para o entendimento da física envolvida e as suas possíveis aplicações concretas. A investigação será restrita à análise das propriedades do estado fundamental destes sistemas.

A partir do comportamento da condutância elétrica em função da temperatura $T$ é possível separar os sistemas condutores em três grupos. No primeiro grupo a resistividade decresce com temperatura até atingir um valor de saturação como ilustrado na figura 1.1, comportamento típico dos metais. O segundo, constituído por sistemas supercondutores, cuja resistência cai abruptamente a zero por baixo de uma temperatura característica, denominada temperatura crítica $T_{c}$, figura 1.2. E finalmente o terceiro e último grupo, constituídos por uma matriz metálica com impurezas magnéticas diluídas, as quais alteram significativamente as suas propriedades a baixas temperaturas, em que a resistividade diminui com o decréscimo da temperatura, atinge um mínimo e torna a crescer, como ilustrado nas figuras 1.3 e 1.4. Os sistemas que estudaremos nesta tese formam parte deste último grupo.

A resistividade de metais puros não magnéticos tende monotonicamente a zero conforme diminui a temperatura do sistema. Apesar de sua dependência ser proporcional a $T$ em um importante intervalo, a medida que a temperatura diminui esta dependência passa a ser $T^{5}$. Ao passo que nos semicondutores não dopados, a resistência segue uma lei do tipo $\exp \left(-E_{g} / k_{B} T\right)$, onde $k_{B}$ é a constante de Boltzmann e $E_{g}$ a energia do gap do semicondutor. Este comportamento é restrito a amostras sem imperfeições na rede cristalina, em que o processo de espalhamento dos elétrons de condução ocorre devido à interação elétronfônon. Podemos explicar heuristicamente como ao reduzir a temperatura, as 


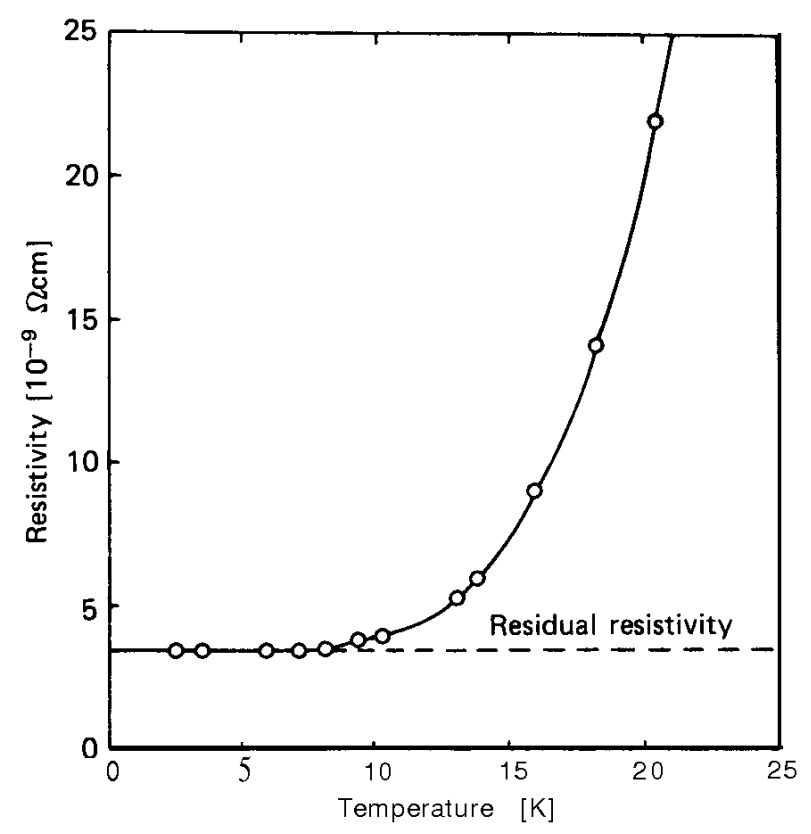

Figura 1.1: Comportamento da resistividade do sódio para diferentes valores da temperatura. Observe a saturação da resistividade a partir dos $10 \mathrm{~K}$, saturação denominada resistividade residual, proveniente dos defeitos na rede cristalina e da interação elétron-fônon [1].

vibrações da rede cristalina, fontes de espalhamento dos elétrons de condução, diminuem e consequentemente a passagem dos elétrons de condução pelo metal é facilitada, resultando em um decréscimo da resistividade. Quando as imperfeições estão presentes, tais como impurezas não magnéticas na matriz metálica ou defeitos, dos quais podemos citar vacâncias livres e quebras de simetrias da rede, a resistividade atinge um valor residual, por volta dos $10 \mathrm{~K}$, como descrito pela regra de Mathiessen e conforme mostrado na figura 1.1 [1]. Aumentando os defeitos da rede, o valor da "resistência de saturação" aumenta, mas a forma da dependência da temperatura permanece a mesma [15].

Todavia, se as impurezas presentes no hospedeiro metálico são magnéticas, e.g., cobalto, então as características do sistema são muito alteradas por sua presença, em especial, observa-se experimentalmente efeitos oriundos do acoplamento do momento magnético da impureza com o spin dos elétrons de condução, alterando as propriedades de transporte, como a resistividade, e o comportamento termodinâmico [7]. São sistemas compostos tipicamente por impurezas de metais de transição $3 \mathrm{~d}$ e terras raras $4 \mathrm{f}$, como uma matriz de cobre com impurezas de ferro, figura 1.3, cério em $\mathrm{LaAl}_{2}$ e em $L a B_{6}$. Ao invés de saturar como no caso dos metais sem impurezas magnéticas, é sabido desde 1930 que a resistividade do metal hospedeiro alcança um mínimo e depois volta 


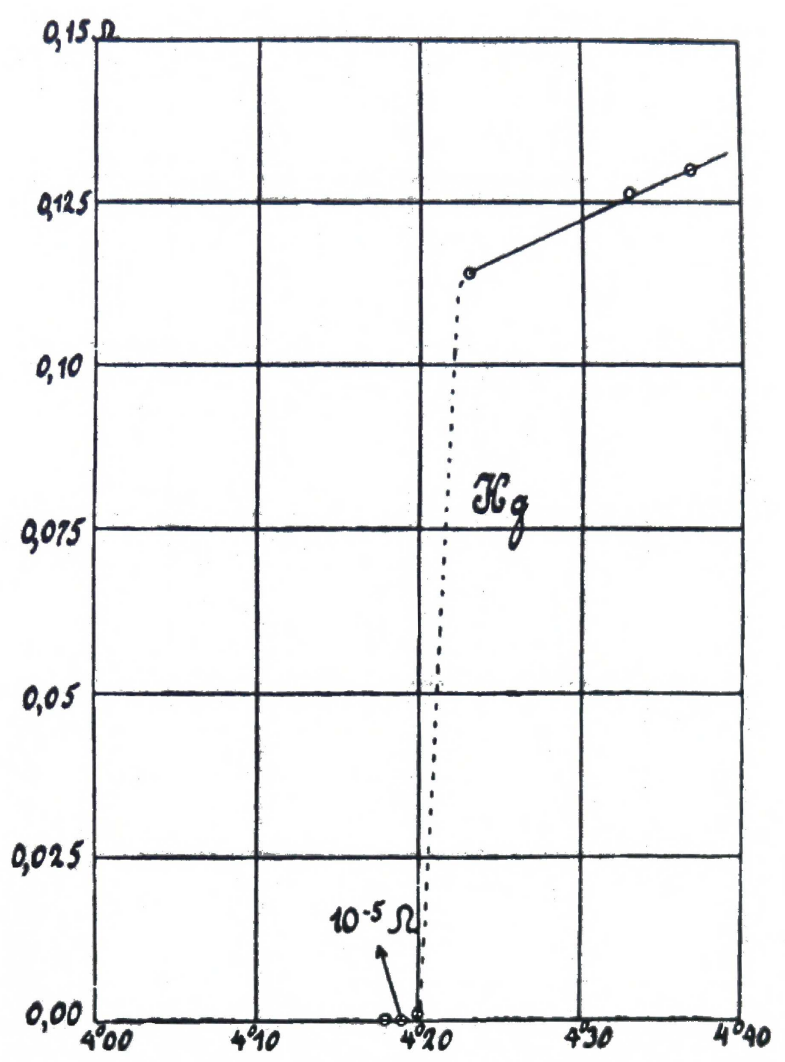

Figura 1.2: Resistência $(\Omega)$ como função da temperatura $(\mathrm{K})$ para o mercúrio, mostrando a transição para o estado supercondutor em $4.20 \mathrm{~K}$. A resistência reduz de cerca de $0.1 \Omega$ a $10^{-5} \Omega$ ao alterar a temperatura em $0.01 \mathrm{~K}$, atingindo, posteriormente, valores imensuráveis $[2,3]$.

a crescer como $-\ln T$ conforme a temperatura $T$ continua a diminuir, como mostramos nas figuras 1.3 e 1.4. Mesmo sem caracterizar uma transição de fase como no caso dos supercondutores, este comportamento e as propriedades eletrônicas em geral do material a baixas temperaturas são determinadas por uma temperatura característica conhecida como temperatura Kondo, $T_{K}$, que aproximadamente pode-se definir como a temperatura em que ocorre a inflexão da resistividade elétrica e esta retorna a crescer ao reduzir a temperatura [15].

Somente em 1964 apareceu uma explicação satisfatória para este comportamento exótico da resistividade. Kondo propôs um modelo no qual o spin dos elétrons de condução interage com o momento magnético das impurezas [8]. Segundo o resultado obtido, o termo de segunda ordem de seu cálculo perturbativo pode ser muito superior ao primeiro, produzindo um aumento logarítmico da resistência com o decréscimo da temperatura, como pode ser visto nas figuras 1.3 e 1.4 .

A teoria de Kondo descreve corretamente o aumento observado na resistividade a baixas temperaturas, todavia prediz incorretamente que a resistividade tende a infinito quando diminuímos ainda mais a temperatura. 


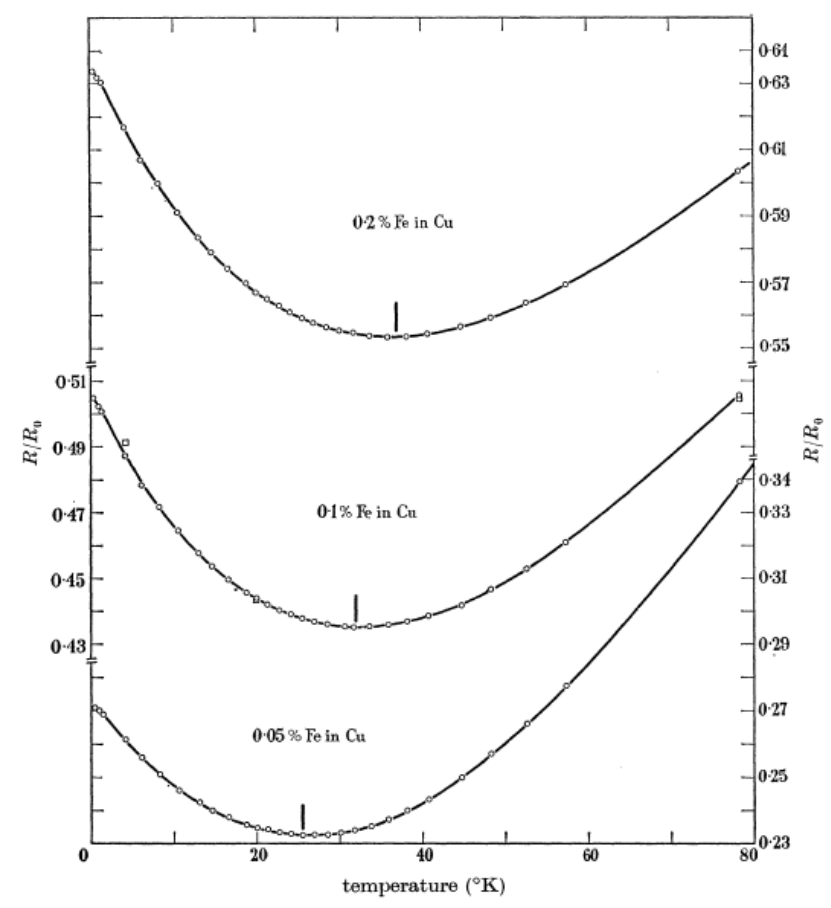

Figura 1.3: Resistência elétrica como função da temperatura para uma liga de cobre e ferro em diferentes concentrações. Note a pequena concentração de ferro, caracterizando-o como uma impureza. As barras indicam a localização do mínimo na resistência, [4]

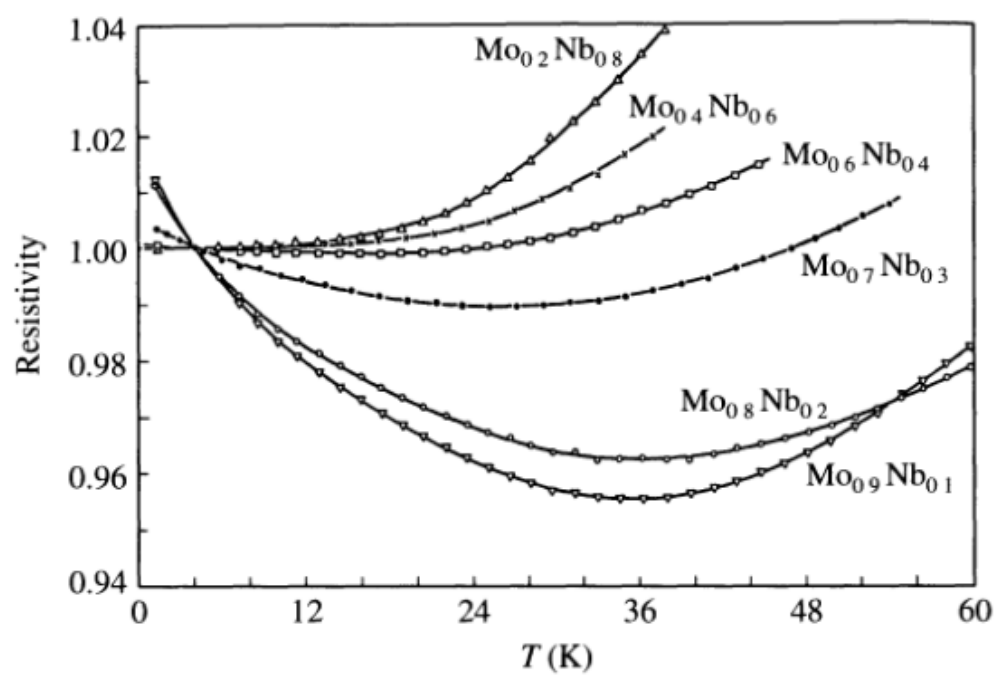

Figura 1.4: Mínimo na resistividade para ligas de molibdênio-nióbio com $1 \%$ de ferro $[5,6]$.

Resulta deste cálculo que a análise de Kondo é correta apenas acima de uma determinada temperatura, a qual ficou conhecida como temperatura Kondo, $T_{K}$.

Somente no final da década de 60 a base teórica para compreender a física 
abaixo de $T_{K}$ surgiu, com a ideia do scaling do problema Kondo proposto por Anderson [16]. O scaling assume que a baixas temperaturas as propriedades físicas são representadas adequadamente por um conjunto finito de graus de liberdade do sistema, modelo coarse-grained, simplificando a descrição do problema. Este modelo pode predizer as propriedades reais do sistema próximo ao zero absoluto, uma vez que diminuindo a temperatura o número de graus de liberdade do sistema é reduzido. Contudo apenas 1974, quando Wilson desenvolveu o método de renormalização numérica [17], capaz de superar as limitações do cálculo perturbativo, foi possível confirmar a hipótese do scaling de Anderson [15]. Seu trabalho demonstrou que a temperaturas inferiores a $T_{K}$ o momento magnético da impureza é completamente blindado pelos spins dos elétrons no metal. Apesar de processos completamente diferentes, podemos estabelecer uma certa analogia com a blindagem da carga elétrica dentro de um condutor [15].

\section{2}

\section{Efeito Kondo}

O efeito Kondo surge quando o momento angular intrínseco ou spin de uma impureza magnética interage com os elétrons do metal hospedeiro, no qual a impureza se encontra [15]. As propriedades de uma impureza magnética modificam-se fundamentalmente na vizinhança da temperatura Kondo, $T_{K}$, o que pode ser observado no comportamento da susceptibilidade magnética $[5,18]$.

As impurezas magnéticas introduzem um termo do tipo Curie-Weiss na susceptibilidade magnética

$$
\chi=\frac{C}{T+\theta},
$$

onde $\theta$ é uma constante com valores típicos da energia térmica, $0<\theta<300 \mathrm{~K}$, complementando a susceptibilidade magnética de Pauli do metal hospedeiro [7]. Quando o sistema se encontra a temperaturas bem superiores a $T_{K}$, a susceptibilidade magnética do sistema obedece a lei de Curie para momentos magnéticos livres. Entretanto, para $T<T_{K}$, a susceptibilidade tende a uma constante, a qual, quando $T=0$, corresponde a um estado singleto que só pode ser polarizado por um campo magnético externo [5, 18]. Enquanto que no ferromagnetismo do volume o magnetismo inicia-se a $T$ menor que a temperatura de Curie, no problema Kondo justamente o oposto ocorre, o magnetismo tende a desaparecer para $T<T_{K}[5]$.

A medida que as impurezas condensam em estados singletos, a dependência da resistividade com $-\ln T$ cessa. A origem do mínimo na resistência é oriunda da interação de troca $J$ entre o spin da impureza e o spin dos elétrons 
de condução.

Em um cálculo perturbativo em $J$, é possível obter a probabilidade de espalhamento dos elétrons de condução do metal hospedeiro pelos momentos magnéticos das impurezas localizadas, permitindo a troca do spin do elétron espalhado e do momento local, o qual age como um centro espalhador. Assim, nestes sistemas, temos duas formas de espalhamento que competem, a interação elétron-fônon que escala com $T^{5}$ e o espalhamento magnético dos elétrons de condução com os momentos magnéticos da impureza. Kondo mostrou ser este espalhamento o responsável pelo termo $-\ln T$ na resistividade elétrica [8], contribuição de segunda ordem em $J$ e divergente conforme $T \rightarrow 0$. Como a resistividade é diretamente proporcional a probabilidade total de espalhamento, a sua principal contribuição quando $T>T_{K}$ é o espalhamento devido à interação elétron-fônon, ao passo que quando $T<T_{K}$ a principal contribuição para resistividade elétrica é a interação de troca entre o spin local e os spins dos elétrons de condução do metal hospedeiro. A contribuição logarítmica do processo de espalhamento que produz a troca do spin impõe o mínimo na resistividade observado $[7,8]$.

Todavia, conforme $T \rightarrow 0$ o termo $\ln T$ diverge e a temperatura em que o elemento de segunda ordem no cálculo perturbativo torna-se comparável ao de primeira ordem, ocorrendo a divergência, ficou conhecida como temperatura Kondo, $T_{K}$, e é a temperatura na qual as propriedades magnéticas do sistema começam a mudar. Como consequência da divergência, a solução de Kondo é válida apenas na região $T \gg T_{K}$.

Novos formalismos foram desenvolvidos com a finalidade de explicar a física envolvida na região $T<T_{K}$. Resultados experimentais mostraram que o momento magnético local não sobrevive em $T=0$. A busca por uma teoria capaz de remover a divergência no tratamento perturbativo proposto por Kondo ficou conhecido como "problema Kondo".

A correlação, observada a partir de experimentos, entre a existência de um termo do tipo Curie-Weiss na susceptibilidade magnética da impureza, caracterizando a presença de um momento magnético local, e a ocorrência do mínimo da resistividade, ver figura 1.4, pavimentou o caminho para o cálculo de Kondo, motivando-o ao cálculo de termos de ordem mais alta na probabilidade de espalhamento dos elétrons de condução por um momento magnético local, cálculo realizado através do modelo s-d. Esta correlação sugere que a interação entre os elétrons de condução com o spin do elétron na impureza origina o mínimo na resistividade.

Kondo também discutiu que o mínimo na resistência, relativo a seu valor em $T=0$, é aproximadamente proporcional à concentração de impurezas, $c_{i m p}$, 
como ilustrado na figura 1.3. Isto é indicativo que o mínimo é resultado de cada impureza atuando independentemente das outras.

É no termo de terceira ordem em $J$ do cálculo perturbativo de Kondo onde aparecem resultados essencialmente diferentes das ordens inferiores. Mostrando que a degenerescência de spin na impureza desempenha um papel significativo no espalhamento dos elétrons de condução.

Considerando todas as contribuições, a resistividade apresenta a forma geral

$$
R(T)=a T^{5}+c_{i m p} R_{0}-c_{i m p} R_{1} \ln \left(\frac{k_{B} T}{D}\right)
$$

onde $a, R_{0}$ e $R_{1}$ são constantes e $D$ a semilargura de banda. O primeiro termo é originário da contribuição dos fônons, o segundo termo é independente da temperatura e o terceiro proveniente do espalhamento dos elétrons de condução pelo momento magnético local.

O mínimo dessa função, de acordo com o observado experimentalmente, está localizado em

$$
T_{\min }=\left(\frac{R_{1}}{5 a}\right)^{\frac{1}{5}} c_{i m p}^{1 / 5}
$$

A temperatura em que o mínimo ocorre é proporcional a $c_{i m p}^{1 / 5}$, e portanto, pouco sensível a flutuações na concentração de impurezas.

A figura 1.5 apresenta a comparação da equação (1-2) com os resultados experimentais para três concentrações de impurezas de ferro em um meio de ouro. Nela, evidencia-se o excelente acordo entre teoria e experimento.

O estado fundamental de um sistema Kondo é um singleto. O termo logarítmico do espalhamento dos elétrons de condução pelo spin da impureza origina um pico estreito na densidade de estados, conhecido como pico Kondo. Ele consiste em uma nova ressonância fixa ao nível de Fermi e todos os elétrons de condução do mar de Fermi contribuem para a formação do nível ressonante. A largura deste pico ressonante, pico Kondo, é proporcional à $T_{K}$. O spin da impureza é blindado pelo spin dos elétrons de condução que ocupam o nível ressonante. Ainda que a energia local da impureza esteja distante do nível de Fermi, o sistema sempre apresentará uma ressonância Kondo no nível de Fermi, desde que a temperatura do sistema esteja abaixo da $T_{K}$.

Apesar de bem estabelecido, e por isso ser um campo de testes usual para o estudo de novas ferramentas numéricas e analíticas de problemas de muitos corpos, o interesse no Efeito Kondo persiste por nos fornecer pistas sobre o entendimento das propriedades eletrônicas de uma grande gama de materiais onde a interação intereletrônica é forte quando comparada as outras interações do sistema.

Desde a explicação deste fenômeno, a atração dos físicos persiste graças aos rápidos avanços nas técnicas experimentais no campo da nanotecnologia 


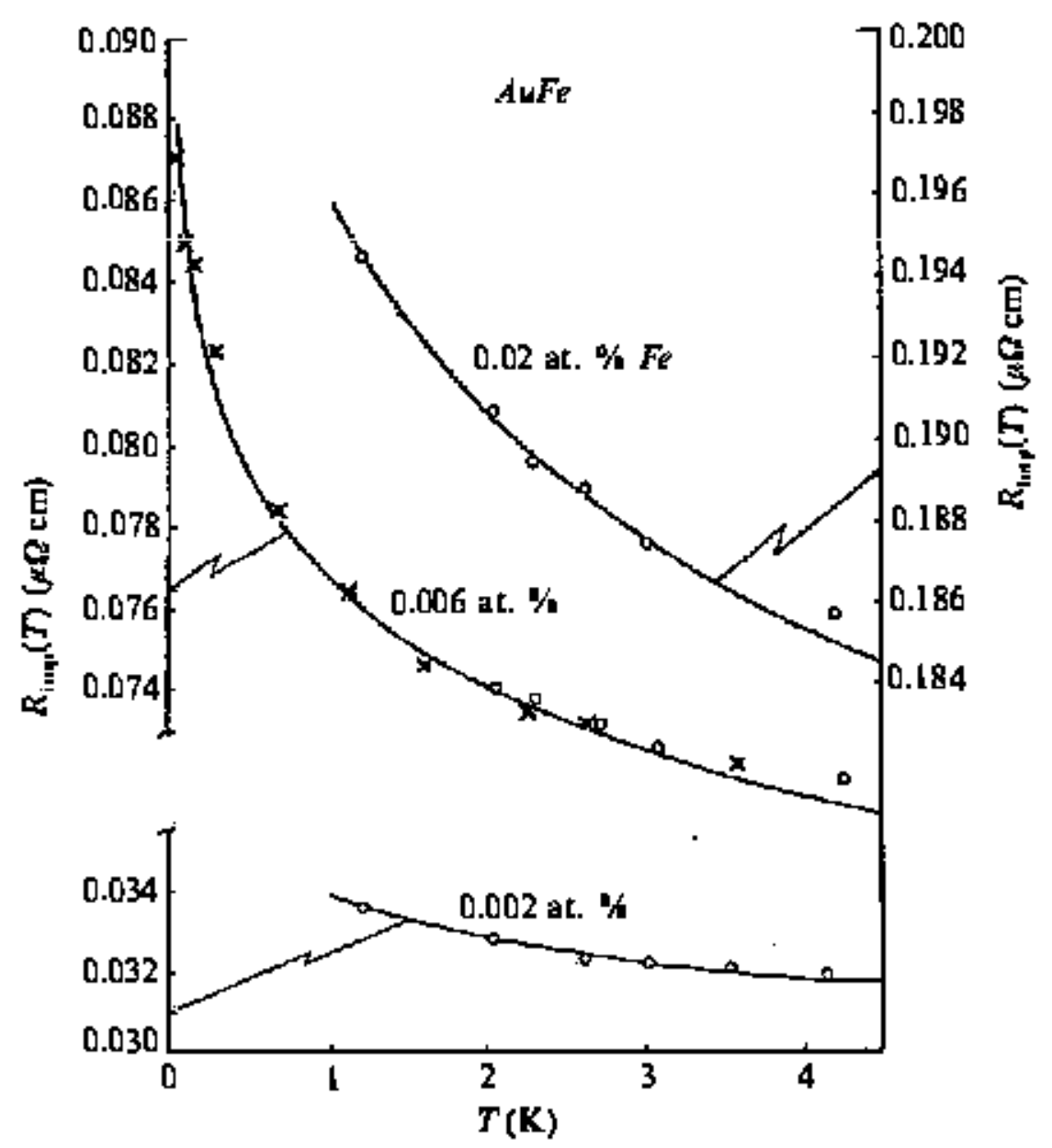

Figura 1.5: Comparação da expressão da resistividade obtida por Kondo com os resultados experimentais. Impurezas de ferro diluídas em uma matriz de ouro $[7,8]$.

que permitiram o controle de sistemas Kondo criados artificialmente. Também foi possível encontrar uma série de aplicações para estes sistemas, algumas das quais constituem o assunto desta tese.

$\mathrm{Na}$ próxima seção discutiremos um destes sistemas que permitem alto controle sobre o Efeito Kondo, os pontos quânticos (PQ). Contrariamente ao efeito Kondo descrito até o momento, em que o transporte eletrônico é dificultado pelo espalhamento dos elétrons de condução por ilhas de momento magnético no volume, resultando no consequente aumento da resistência elétrica, o efeito Kondo em estruturas de PQ, como os que serão analisados nesta tese, cria um canal permitindo a passagem dos elétrons devido à ressonância no nível de Fermi. Assim, deste ponto de vista, se por um lado o efeito Kondo no volume é deletério ao transporte eletrônico, por outro, ele apresenta-se como uma ferramenta interessante para facilitar a circulação dos elétrons em sistemas nanoscópicos, como os construídos com PQ. 


\section{3 \\ Pontos quânticos}

O ponto quântico (PQ) é um dispositivo capaz de aprisionar os elétrons, desde um único elétron até milhares, em espaços restritos, cujas dimensões típicas vão dos nanômetros a poucos micrômetros. O tamanho, a forma e as interações dos $\mathrm{PQs}$ podem ser controlados com grande precisão, graças aos avanços na tecnologia de nanofabricação, permitindo o estudo de problemas fundamentais na física, como o Efeito Kondo [19]. Eles podem ser construídos por processos de litografia e, com suas dimensões nanoscópicas, pode apresentar características típicas dos átomos com propriedades que podem ser modificadas artificialmente. Em particular é possível confinar um número definido de elétrons acoplados por tunelamento quântico a elétrons deslocalizados fora do PQ, tornando este dispositivo um transistor de um elétron [10], já que é possível controlar o número de elétrons um a um dentro dele através de potenciais de porta.

Devido às suas propriedades eletrônicas assemelharem-se com os átomos, os PQs podem ser considerados átomos artificiais. Contudo, diferentemente dos átomos reais eles permitem um alto controle sobre seus parâmetros através das voltagens aplicadas nas portas do PQ, propiciando a análise de "átomos" com spin total nulo, ou não, apenas alterando o número de elétrons confinados no dispositivo, equivalendo a alterar as características da impureza com grande praticidade [19].

Através do tunelamento eletrônico, muitas propriedades dos PQs têm sido estudadas. O tunelamento pode ser controlado nos PQs pela repulsão Coulombiana entre os elétrons presentes no PQ. Com uma barreira de potencial relativamente alta em relação aos outros parâmetros do sistema, o tunelamento de elétrons pode ser fraco o suficiente para que um número finito de elétrons fiquem aprisionados no PQ. Ao alterar o número de elétrons presentes no PQ, a energia contida no PQ muda devido aos novos estados localizados que são ocupados ou desocupados e à interação Coulombiana. Portanto, não haverá corrente no circuito até que o potencial de porta forneça a energia extra necessária para o elétron adicional poder popular o PQ. Este regime é conhecido como bloqueamento de Coulomb [19]. A figura 1.6 ilustra este processo de povoamento do PQ, onde nos vales o número de elétrons no PQ é fixo. Ao aumentar o potencial de porta e passar completamente por um pico, o número de elétrons armadilhados no PQ é aumentado em um. Entretanto, quando o potencial de porta é tal que o valor coincide com o pico, o sistema está em um regime de flutuação de carga, um crossover entre as duas configurações estáveis, a primeira com um número definido de elétrons e a outra com um 
elétron a mais. Esta flutuação no número de elétrons faz possível a corrente fluir pelo circuito e resulta nos picos observados na condutância [9, 20].

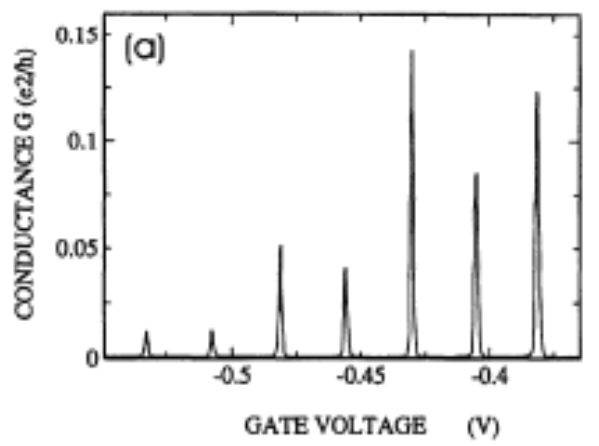

Figura 1.6: Condutância através do PQ como função do potencial de porta, a temperatura de $10 \mathrm{mK}$ [9]. Da esquerda para a direita, o primeiro pico corresponde a voltagem em que um elétron entra no PQ. E a cada novo pico subsequente, um novo elétron entra no PQ.

$\mathrm{O}$ alto controle no confinamento de elétrons no PQ, o que permite caracterizar ele como um transistor de um elétron, confere ao PQ um ambiente natural para o estudo do efeito Kondo.

Apresentamos na figura 1.7 um PQ de GaAs/AlGaAs com várias portas metálicas, sendo possível armadilhar um gás bidimensional de elétrons entre duas camadas com propriedades eletrônicas distintas. Na região de confinamento deste $\mathrm{PQ}$, cerca de 50 elétrons podem ser aprisionados, permitindo que o efeito Kondo torne-se visível quando a temperatura for menor que a $T_{K}$ do sistema.

Para a existência do efeito Kondo é necessário que o sistema possua pelos menos dois estados degenerados (simetria $\mathrm{SU}(2)$ ), como por exemplo a degenerescência oriunda do spin. Se sobre este sistema atua um campo magnético externo, podemos, aumentando gradativamente o seu valor, afastar o sistema da região de parâmetros em que o efeito Kondo existe. Devido ao campo, as energias dos elétrons confinados no PQ e a densidade de estados do gás de Fermi são deslocadas pelo efeito Zeeman, separando os diferentes estados de spin e quebrando a simetria $\mathrm{SU}(2)$.

A figura 1.8 apresenta uma série de gráficos da condutância diferencial para um sistema em que uma diferença de potencial muito pequena é aplicada entre dois contatos separados por um PQ e que o efeito Kondo é utilizado para permitir o transporte dos elétrons através do PQ, segundo a discussão efetuada nesta seção. Quando a temperatura é inferior a $T_{K}$, a ressonância Kondo no nível de Fermi permite a passagem dos elétrons pelo PQ, resultando no pico na condutância diferencial como mostramos no primeiro painel superior da 


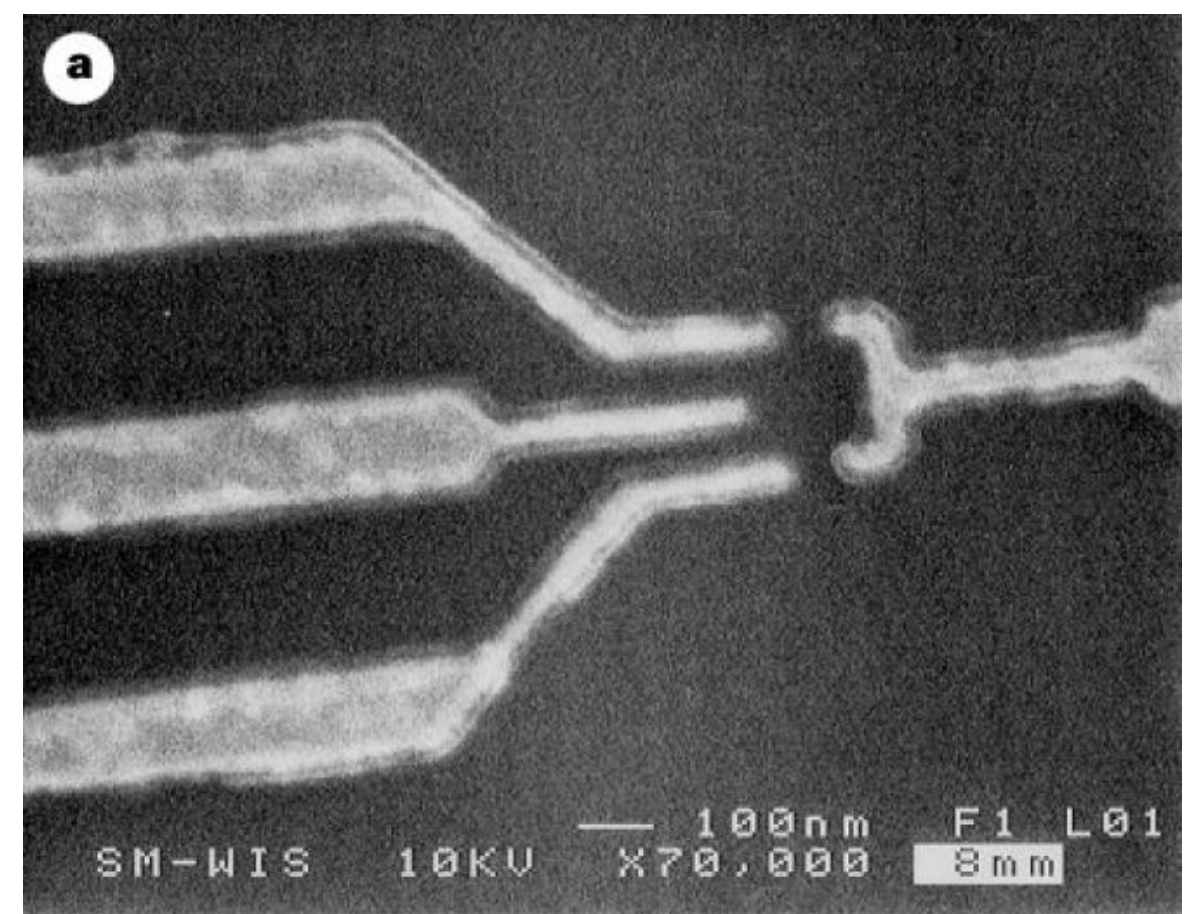

Figura 1.7: Imagem de um PQ obtida a partir do escaneamento de microscopia eletrônica [10].

esquerda. Na ausência de campo magnético, os gráficos da coluna da esquerda na figura 1.8 apresentam a mudança da condutância diferencial local sobre o PQ conforme a temperatura vai mudando de $90 \mathrm{mK}, 300 \mathrm{mK}$ até 600 mK. É possível observar para baixas temperaturas, $T<T_{K}$, a existência de um pico, o pico Kondo, que desaparece para o maior valor, $T>T_{K}$. Outro comportamento importante pode ser estudado variando a intensidade do campo magnético externo com a temperatura fixa. Iniciando do gráfico no painel superior esquerdo é possível observar que conforme a intensidade do campo aumenta o pico central é separado pela ação do campo magnético [10]. 


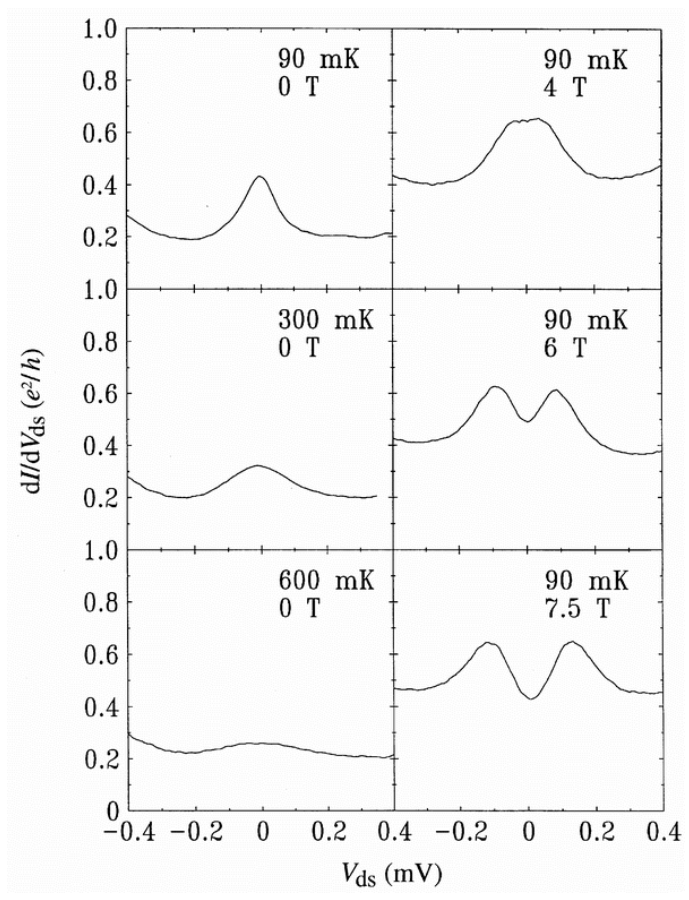

Figura 1.8: Condutância diferencial. Observe nos painéis da esquerda que a medida que a temperatura aumenta de $90 \mathrm{mK}$ a $600 \mathrm{mK}$ o pico na condutância diminui até sumir. Nos painéis da direita observamos a separação dos picos com o aumento do campo para $90 \mathrm{mK}$, assinatura do Efeito Kondo [10].

\section{4}

\section{Modelo da Impureza de Anderson}

Com a finalidade de descrever a física de uma impureza magnética interagindo com os elétrons de condução, analisamos a solução do modelo de Anderson [7]. Este é o Hamiltoniano básico usado para descrever as propriedades físicas dos sistemas que serão estudados nesta tese. Faremos aqui somente uma apresentação das suas propriedades mais importantes. Nos próximos capítulos iremos aprofundar na discussão do modelo, na medida que se faça necessária para descrever os sistemas em análise. De uma maneira geral, uma impureza em um metal hospedeiro pode ser descrita a partir de um Hamiltoniano que apresenta $N_{0}$ elétrons e suas interações, como o mostrado a seguir,

$$
H=\sum_{i=1}^{N_{0}}\left(\frac{\boldsymbol{P}_{i}^{2}}{2 m}+V\left(\boldsymbol{r}_{i}\right)+V_{i m p}\left(\boldsymbol{r}_{i}\right)\right)+\frac{1}{2} \sum_{i \neq j}^{N_{0}} \frac{e^{2}}{\left|\boldsymbol{r}_{i}-\boldsymbol{r}_{j}\right|},
$$

onde o primeiro termo, entre parênteses, descreve a energia cinética de um elétron com momento $\boldsymbol{P}$ e massa $m$, o potencial periódico devido ao núcleo dos átomos que compõem a rede do metal hospedeiro $V$ (antes de incluir a impureza) e o potencial adicional devido ao núcleo da impureza $V_{i m p}$. Ao passo que o segundo termo representa a interação Coulombiana entre os elétrons com 
carga e. A busca de uma solução de primeiros princípios para esse Hamiltoniano apresenta-se complicada, devida à interação Coulombiana.

Para o sistema que desejamos modelar, o metal hospedeiro é representado por uma banda de condução, como as oriundas de estados s e p. Os elétrons de condução nestas bandas são essencialmente partículas livres movendo-se em um potencial periódico (a interação Coulombiana de longo alcance é blindada, os elétrons junto à nuvem oriunda desta blindagem podem ser considerados como quasi-partículas). As interações de curto alcance entre as quasi-partículas devem ser pequenas para uma banda de condução ampla, pois os estados estão mais deslocalizados que em bandas estreitas. Além disso, o tempo de vida de partículas próximas nível de Fermi é muito grande como é conhecido da teoria de líquido de Fermi [7]. Assim, pelas considerações argumentadas, é razoável admitir a aproximação que despreza as interações entre as quasi-partículas e descreve os elétrons de condução do metal hospedeiro por um Hamiltoniano de um elétron, primeiro termo do Hamiltoniano de Anderson na equação (1-5).

Em uma primeira abordagem é razoável supor que os elétron na impureza comportam-se como partículas independentes como no caso do metal hospedeiro e introduzir um potencial efetivo originário da presença da impureza. Todavia, uma análise mais cuidadosa da impureza mostra que esta visão não está correta. Se a impureza possui carga proveniente do metal hospedeiro, então existirá um potencial Coulombiano. Contudo, da teoria do eletromagnetismo clássico, sabemos que não existe campo elétrico macroscópico no metal. A carga deve ser blindada a nível atômico pelos elétrons de condução. Outro aspecto importante é que o potencial da impureza pode ser forte o suficiente para induzir um estado ligado próximo a ela e que terá uma função de onda que cai exponencialmente com a distância da impureza. Ao passo que um potencial fraco para produzir um estado ligado pode, contudo, localizar os elétrons de condução por um tempo nas proximidades da impureza. Isto acontece pois surge um pico fino na densidade de estados dos elétrons de condução - conhecido como ressonância de estados virtuais — devido ao espalhamento no sítio do impureza, onde os elétrons de condução permanecem por um tempo, mas como são funções de onda de Bloch, estes estados não são ligados. Como esta ressonância ocorre na vizinhança do nível de Fermi, está claro que ela influencia nas propriedades do sistema como calor específico e susceptibilidade magnética [7].

As ressonâncias de estados virtuais ocorrem para impurezas de metais de transição ou terras raras quando os níveis d ou f encontram-se dentro da banda de condução dos elétrons da matriz metálica. Como as funções de onda destes estados são muito localizadas, ver figura 1.9, estes estados funcionam 
como uma barreira de potencial que pode ser tunelada pelos elétrons. Estes estados funcionam como estados ligados virtuais.

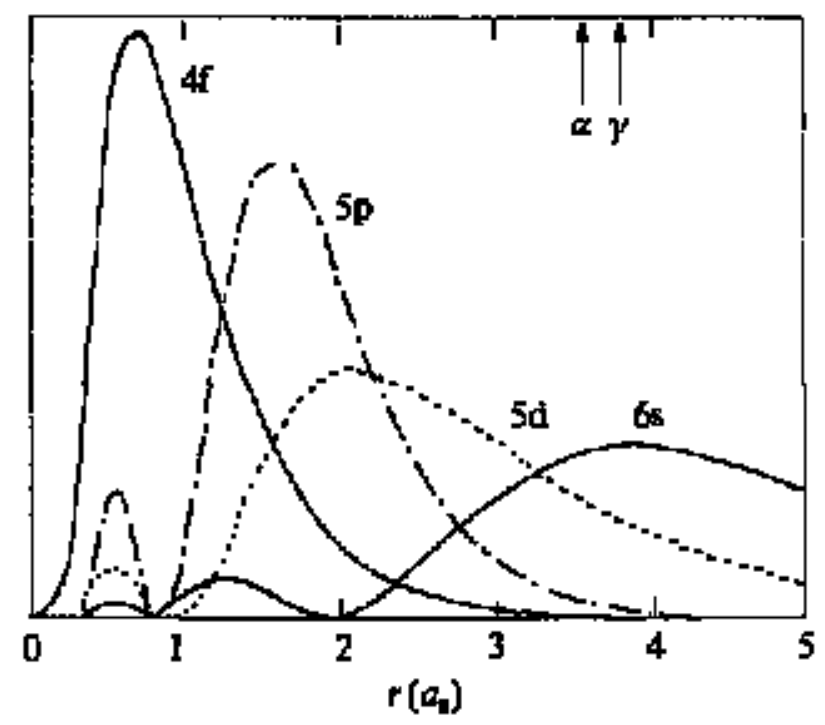

Figura 1.9: Funções de onda do cério obtidas pelo cálculo de densidade funcional para a configuração $4 \mathrm{f}^{1} 5 \mathrm{~d}^{1} 6 \mathrm{~s}^{2}[7]$. As setas no canto superior direito indicam o tamanho do raio da célula de Wigner-Seitz para $\alpha$ e $\gamma$-Ce. Observe como o estado $4 \mathrm{f}$ é mais localizado que a célula de Wigner-Seitz e que os outros estados.

Existe uma sobreposição ou elemento de matriz de hibridização $V_{\boldsymbol{k}}$ entre os estados de Bloch dos elétrons de condução e a impureza, dando origem ao segundo termo do Hamiltoniano de Anderson, equação (1-5). Por sua vez, a impureza é descrita por uma energia local $\epsilon_{0 \sigma}$ e a interação Coulombiana, terceiro e quarto termos da mesma equação.

Depois de descrever o significado e a origem física de cada termo, excetuando a interação intereletrônica que faremos a seguir, finalmente apresentamos o Hamiltoniano do modelo de Anderson, dado por

$$
H=\sum_{\boldsymbol{k}, \sigma} \epsilon_{\boldsymbol{k} \sigma} c_{\boldsymbol{k} \sigma}^{\dagger} c_{\boldsymbol{k} \sigma}+\sum_{\boldsymbol{k}, \sigma}\left(V_{\boldsymbol{k}} c_{0 \sigma}^{\dagger} c_{\boldsymbol{k} \sigma}+V_{\boldsymbol{k}}^{*} c_{\boldsymbol{k} \sigma}^{\dagger} c_{0 \sigma}\right)+\sum_{\sigma} \epsilon_{0 \sigma} n_{0 \sigma}+U n_{0 \uparrow} n_{0 \downarrow}
$$

onde $\epsilon_{\boldsymbol{k} \sigma}$ é o elemento diagonal dos elétrons presentes no mar de Fermi, $c_{\boldsymbol{k} \sigma}^{\dagger}$ $\left(c_{\boldsymbol{k} \sigma}\right)$ o operador de criação (aniquilação) de um elétron no mar de Fermi com momento $k$ e spin $\sigma, V_{k}$ é a hibridização entre a impureza e o mar de Fermi, $c_{0 \sigma}^{\dagger}\left(c_{0 \sigma}\right)$ o operador de criação (aniquilação) de um elétron na impureza, $U$ a repulsão Coulombiana dos elétrons na impureza e $n_{0 \sigma}=c_{0 \sigma}^{\dagger} c_{0 \sigma}$.

Os estados dos elétrons de condução e os localizados não são ortogonais. Entretanto, para estados localizados d ou f em uma banda de condução com estados s ou p é razoável desprezar esta não ortogonalidade e considerá-los ortogonais. 
$\mathrm{Na}$ formulação de primeiros princípios, a interação intereletrônica na impureza apresenta a forma

$$
U=\int \phi_{d}^{*}(\boldsymbol{r}) \phi_{d}^{*}\left(\boldsymbol{r}^{\prime}\right) \frac{e^{2}}{\left|\boldsymbol{r}-\boldsymbol{r}^{\prime}\right|} \phi_{d}(\boldsymbol{r}) \phi_{d}\left(\boldsymbol{r}^{\prime}\right) d \boldsymbol{r} d \boldsymbol{r}^{\prime},
$$

onde $\phi_{d}(\boldsymbol{r})$ é a função de onda de um elétron no orbital d da impureza o qual encontra-se na posição $\boldsymbol{r}$ interagindo com o outro elétron na posição $\boldsymbol{r}^{\prime}$. A interação Coulombiana nas camadas d podem atingem valores da ordem de $30 \mathrm{eV}$. Contudo, devido à deslocalização parcial dos orbitais d da impureza, ao meio metálico e à blindagem oriunda dos outros elétrons, o valor da interação é estimado entre 1 e $7 \mathrm{eV}$ [7]. Para o caso de impurezas compostas por terras-raras, o mesmo raciocínio é válido. Porém, como os orbitais 4f são mais localizados, figura 1.9, esta interação possui um valor maior.

A interação Coulombiana no modelo de Anderson é introduzida pelo último termo da equação (1-5), onde os orbitais d são considerados não degenerados a fim de simplificar o tratamento, permitindo no máximo apenas dois elétrons popularem a impureza devido à degenerescência de spin. Trata-se de um termo de muitos corpos envolvendo elétrons com spins diferentes.

No modelo de Anderson como tratamos até aqui, não existe um termo de interação entre as impurezas, pois como mencionado na seção 1.2, a baixa concentração das mesmas serve como argumento para que as impurezas estejam longe o suficiente uma da outra na matriz metálica para não incorporarmos este termo. Porém, como ficará claro ao decorrer da tese, para tratar o sistema de dois PQ acoplados capacitivamente teremos uma repulsão Coulombiana entre duas impurezas de Anderson, pois, neste contexto, o sistema é propositalmente construído com os dois $\mathrm{PQ}$ próximos, modelados pelas impurezas aqui descritas.

Alguma informação relevante deste modelo pode ser extraída na aproximação de limite "atômico" com $V_{\boldsymbol{k}}=0$, o que significa que os elétrons de condução não estão acoplados aos estados da impureza, tornando sua solução simples. Neste caso, considerando apenas a contribuição da impureza à energia do estado fundamental do sistema $E$, existem três configurações possíveis para os elétrons na impureza: a primeira quando a impureza não possui nenhum elétron $(E=0)$, a segunda quando apenas um elétron encontra-se na impureza $\left(E=\epsilon_{0}\right)$ e a terceira quando existem dois elétrons na impureza $\left(E=2 \epsilon_{0}+U\right)$. Destas três configurações, a única que apresenta degenerescência de dois níveis correspondendo ao spin - e portanto possui um momento magnético associado e contribui com um termo de Curie a susceptibilidade magnética - é a configuração com apenas um elétron na impureza. Para que esta situação ocorra é necessário que $\epsilon_{0}<\epsilon_{F}$, onde $\epsilon_{F}$ é o nível de Fermi do sistema, para 
que um elétron ocupe a impureza e $\epsilon_{0}+U>\epsilon_{F}$ para que o segundo elétron não esteja na impureza, garantindo a ocupação simples dela.

Entretanto, o interesse reside na situação em que o acoplamento entre a impureza e os elétrons de condução não seja nulo. Com $V_{\boldsymbol{k}}$ pequeno é possível estabelecer uma relação entre o modelo de Anderson e os modelos s-d usando teoria de perturbação na ordem mais baixa, obtido primeiramente por Schrieffer e Wolff em 1966 [7, 21]. A relação entre o acoplamento de troca efetivo $J_{\boldsymbol{k}, \boldsymbol{k}^{\prime}}$ e os parâmetros do modelo de Anderson é

$$
J_{k, k^{\prime}}=V_{k}^{*} V_{k^{\prime}}\left(\frac{1}{\epsilon_{0}+U-\epsilon_{k^{\prime}}}+\frac{1}{\epsilon_{k}-\epsilon_{0}}\right)
$$

que é válida para $\left|\epsilon_{\boldsymbol{k}}\right| \ll\left|\epsilon_{0}-\epsilon_{F}\right|$ e $\left|\epsilon_{\boldsymbol{k}}\right| \ll\left|U+\epsilon_{0}-\epsilon_{F}\right|$. No regime em que o sistema tem momento magnético local, $\epsilon_{0}+U>\epsilon_{F}$ e $\epsilon_{0}<\epsilon_{F}$, o acoplamento de troca efetivo entre o spin dos elétrons de condução e o spin localizado na impureza é antiferromagnético para elétrons de condução na região do nível de Fermi, $\epsilon_{\boldsymbol{k}} \sim \epsilon_{\boldsymbol{k}^{\prime}} \sim \epsilon_{F}$. Portanto, quando $\epsilon_{0}<\epsilon_{F}<\epsilon_{0}+U$ o termo de troca efetivo entre os elétrons de condução e o spin localizado na impureza é antiferromagnético.

O Hamiltoniano da impureza de Anderson foi resolvido por uma série de abordagens diferentes, tanto numéricas como a logarithmic discretization embedded cluster approximation (LDECA) [22], o numerical renormalization group (NRG) [17], o density matrix renormalization group (DMRG) [23] ou mais algébricos como projection operator approach (POA) [24-26], mean field slave bosons approximation (MFSBA) [27-33], non-crossing approximations (NCA) [34, 35], one-crossing approximations (OCA) [36], Monte Carlo quântico [37], equações de movimento das funções de Green [38] e Bethe Ansatz [39]. Dentre os métodos citados, podemos considerar o LDECA, o NRG e o POA como numericamente exatos para descrever a física envolvida, enquanto os outros envolvem aproximações com diferentes características.

A escolha do Hamiltoniano de Anderson em detrimento ao modelo de Kondo deve-se ao primeiro descrever uma física mais abrangente, com a possibilidade de flutuação de carga na impureza, que determina a energia de hibridização, ao passo que no modelo Kondo inclui apenas interações entre spins.

Quando a $T \gg T_{K}$, o sistema é bem descrito pela aproximação onde a densidade de estados apresenta dois picos lorentzianos localizados em $\epsilon_{0}$ e $\epsilon_{0}+U$, conhecida como aproximação Hubbard III [40]. A medida que a temperatura é reduzida, uma nova física emerge, a qual não pode ser descrita por esta aproximação. Um mecanismo de espalhamento entre o spin da impureza e dos elétrons de condução começa a governar as propriedades 
do sistema, resultando no pico Kondo na densidade de estados de largura proporcional à $T_{K}$, que define uma escala de energia na qual as propriedades do sistema mudam consideravelmente. A figura 1.10 mostra a ressonância Kondo descrita.

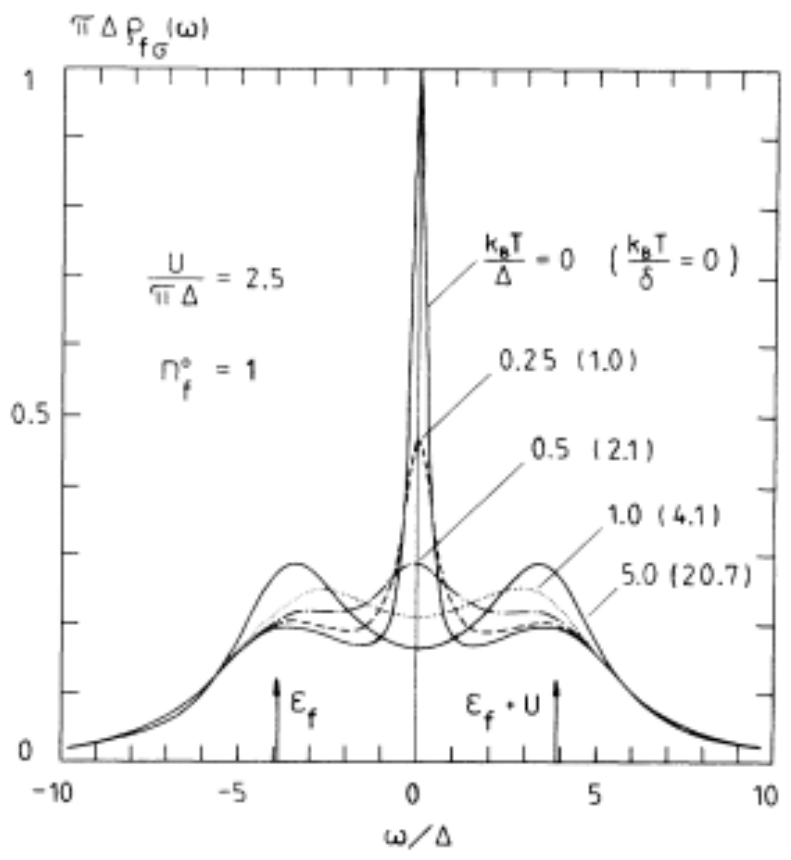

Figura 1.10: Densidade espectral, $\pi \Delta \rho(\omega)$, para o modelo de Anderson simétrico com $U / \pi \Delta=2.5$ e ocupação $\left\langle n_{0}\right\rangle=1$ [11]. Quando o sistema está no regime Kondo, o sistema apresenta, além dos picos em $\epsilon_{0}$ e $\epsilon_{0}+U$, o pico no nível de Fermi. A largura do pico a meia altura é similar ao valor da $T_{K}$.

A temperatura Kondo, $T_{K}$, relaciona-se com os parâmetros do modelo de Anderson (semilargura de banda $D, \epsilon_{0}, U$ e função de hibridização $\Delta=$ $\pi V^{2} \rho\left(\epsilon_{F}\right)$, onde $\rho$ é a densidade de estados da banda de condução) através da expressão de Haldane [41, 42],

$$
T_{K}=\sqrt{\Delta D} \exp \left(\frac{\pi \epsilon_{0}\left(\epsilon_{0}+U\right)}{2 \Delta U}\right),
$$

que estabelece uma lei de escala, apesar de não haver um consenso quanto a expressão concreta do pré-fator, que na fórmula de Haldane é $\sqrt{\Delta D}$.

\section{5}

\section{Objetivos e sistemas analisados}

Investigamos nesta tese as propriedades de sistemas modelados pelo Hamiltoniano de Anderson, principalmente as relacionadas ao transporte eletrônico, as quais são caracterizadas a partir do ponto de vista da condutância do sistema. 
A fim de encontrar a energia do estado fundamental deste Hamiltoniano, usaremos o formalismo de operadores de projeção (POA), acrônimo de projection operator approach [24-26], que é discutido em detalhes no apêndice A.

De posse deste formalismo, estenderemos-o à análise do Hamiltoniano de Anderson com duas impurezas, também no apêndice A. Extensão necessária para estudar as propriedades de um filtro de spin e a transição entre os efeitos Kondo de simetria SU(4) e SU(2), no capítulo 2.

O capítulo 3 é reservado ao estudo da interação spin-órbita no Hamiltoniano de Anderson e analisar os efeitos desta interação na temperatura Kondo. No seguinte, capítulo 4, investigamos um sistema de um PQ acoplado ao mar de Fermi com interação spin-órbita. Propomos utilizar este sistema como um transistor de spin conforme será caracterizado ao decorrer do capítulo.

A tese será concluída com um capítulo de conclusões gerais possíveis de serem obtidas dos estudos realizados. Por último, faremos uma apresentação parcial de uma metodologia que permitiria estender o método dos operadores de projeção ao estudo de sistemas com interação Coulombiana no volume. Estas ideias serão apresentadas com o intuito de indicar desdobramentos possíveis a serem desenvolvidos no futuro. 


\section{Transição SU(4)-SU(2) e propriedades de filtro de spin de um nanossistema de dois pontos quânticos}

\section{1 \\ Introdução}

Podemos encontrar na literatura, em especial mais recentemente, muita pesquisa relacionada ao efeito Kondo em nanoestruturas que apresentam graus de liberdade orbital degenerados em adição aos do spin, cuja simetria pertence ao grupo de Lie $\mathrm{SU}(N)$, para $N \geq 2$. Sistemas como o transistor de um átomo [43], nanotubos de carbono [44] e dois pontos quânticos (DPQ) acoplados capacitivamente [45] são tidos exemplos de sistemas com $N=4$. Diversos estudos foram desenvolvidos relativos a transição $\mathrm{SU}(4)-\mathrm{SU}(2)$ [46], produzido pela quebra de simetria $\mathrm{SU}(4)$ quando existe uma diferença do potencial de porta $\epsilon_{0}$ aplicada a cada PQ ou quando os contatos são conectados por diferentes elementos de matriz $V$. Além disso, manipulando os parâmetros do sistema, sem explicitamente restaurar a simetria destruída, mostrou-se que o estado fundamental apresenta a simetria SU(4) como uma propriedade emergente [47, 48]. Cabe comentar que a referência [49] mostrou que as conclusões obtidas por Tosi et al. [47] acerca da simetria SU(4) emergente são obtidas assintoticamente se as repulsões Coulombianas intraponto e interponto são maiores que a semilargura de banda $D$ [50].

Por fim, estudos teóricos recentes de dispositivos de dois pontos quânticos (DPQ) conectados a dois canais independentes e sobre efeito de um campo magnético externo exibiram um estado Kondo SU(2) exótico, com propriedades de correntes de spin polarizadas através de cada PQ [51].

Neste capítulo, discutiremos dois assuntos principais: (i) a transição Kondo SU(4)-SU(2) devido a um campo magnético externo em um sistema de dois PQ capacitivamente acoplados e (ii) as propriedades de filtro de spin associadas a este dispositivo que emergem da transição na vizinhança do estado $\mathrm{SU}(2)$. Apesar de alguns aspectos relacionados ao problema já foram analisados(veja referência [52] para (i), referências [51,53] para (ii) e referências neles contidas), propriedades importantes desta transição ainda não foram estudadas e serão discutidas neste capítulo. 
Capítulo 2. Transição SU(4)-SU(2) e propriedades de filtro de spin de um nanossistema de dois pontos quânticos

A transição $\mathrm{SU}(4)-\mathrm{SU}(2)$ é controlada no nosso caso pelo campo magnético externo, originando um desdobramento em energia entre os estados de diferentes spins devido ao efeito Zeeman e, consequentemente, reduzindo a simetria do Hamiltoniano de SU(4) para SU(2). Todavia, apesar da presença de um campo magnético finito, nosso resultado mostra que a simetria do estado fundamental muda de $\mathrm{SU}(4)$ para $\mathrm{SU}(2)$ quando o potencial de porta $\epsilon_{0}$ aplicado em ambos os PQs é reduzido. O estado fundamental deste sistema apresenta a simetria SU(4), conhecida como Kondo SU(4) emergente do estado fundamental [48], é um resultado interessante por si só, visto que o Hamiltoniano possui a simetria $\mathrm{SU}(2)$.

Como mostraremos ao longo do texto, mais precisamente na seção 2.4, a transição $\mathrm{SU}(4)-\mathrm{SU}(2)$ pode ser estudada calculando os valores da ocupação para as diferentes polarização de spin, i.e., a diferença $\left\langle n_{\uparrow}\right\rangle-\left\langle n_{\downarrow}\right\rangle$, obtida no estado fundamental do sistema de DPQ, cujo papel é similar a um parâmetro de ordem que define uma transição entre duas fases, embora, neste caso, estamos lidando com um processo de crossover e não uma transição de fase no contexto usual. Para um campo magnético fixo, que produz o desdobramento Zeeman, a transição é caracterizada como ocorrendo para um potencial de porta máximo $\epsilon_{0}^{\max }$ onde a ocupação eletrônica de spin para baixo, $\left\langle n_{\downarrow}\right\rangle$, atinge seu valor máximo, que pode ser bem caracterizado, nomeado como $\left\langle n_{\downarrow}\right\rangle^{\max }$, presente na figura 2.3. Também nomearemos $B_{\max }$ como o do desdobramento Zeeman em que ocorre este máximo. Analisaremos a dependência funcional entre $B_{\max } \mathrm{e}$ $\epsilon_{0}^{\max }, B_{\max }=f\left(\epsilon_{0}^{\max }\right)$, onde verifica-se que dentro do regime Kondo $f$ tem um comportamento universal e similar à temperatura Kondo $T_{K}$ como função do potencial de porta. Contudo, deve-se notar que a transição, como definido aqui, ocorre mesmo quando o sistema está dentro da região do regime de flutuação de carga. Neste caso, não se pode dizer, rigorosamente falando, que que o processo aconteça no regime Kondo. A existência clara deste máximo, independentemente do regime em que o sistema se encontra, permite que $B_{\max }$ seja caracterizado como uma escala de energia que controla a transição.

No que diz respeito às propriedades de filtro de spin (ii) do sistema de dois PQs tratado aqui, nosso resultado mostra que a ocupação eletrônica está polarizada no spin devido ao campo magnético. Sobre a temperatura e o campo magnético mínimos necessários para o funcionamento adequado do dispositivo de filtro de spin, temos que $B_{\max }$ é muito inferior a temperatura Kondo SU(4) do sistema, $T_{K} S U(4)$. Sendo assim, o dispositivo pode operar a temperaturas próximas a $10 \mathrm{~K}$ e campo magnético de aproximadamente 0.1 Tesla. Estes dois fatos nos permite afirmar que o dispositivo analisado possui propriedades de filtro de spin bastante eficientes, que serão discutidas ao decorrer do capítulo. 
Nesta tese, tal fenômeno é investigado usando o formalismo de operadores de projeção (POA), da sigla em inglês projector operator approach, o qual mostrou-se capaz de descrever, de forma quase exata, as propriedades estáticas do estado fundamental do Hamiltoniano da Impureza de Anderson [24, 25], como pode ser visto mais claramente na Ref. [24], onde os resultados dos POA concordam muito bem com os obtidos pelo Bethe ansatz [39]. O resultado obtido é então confrontado com o oriundo da aproximação de campo médio dos bósons escravos (MFSBA) — também acrônimo da sigla em inglês mean-field slave bosons approximation [28-33]. Observe que originalmente o formalismo dos POA foi desenvolviado para o estudo de problemas Kondo com apenas uma impureza. Para a análise do sistema de dois PQ capacitivamente acoplados nós estendemos o formalismo para incluir as duas impurezas. Assim como no caso de apenas uma impureza, esta extensão permite o cálculo das propriedades do estado fundamental a temperatura zero de forma quase exata. A comparação dos resultados pelas diferentes abordagens, POA e MFSBA, permite identificar uma concordância qualitativa e semiquantitativa na descrição física do sistema [12].

O presente capítulo é constituído por: a seção 2.2 onde nós descrevemos o sistema de dois PQs capacitativamente acoplados e a seção 2.3 na qual apresentamos o formalismo de POA estendido para o estudo de dois PQs e utilizado no estudo das propriedades do sistema. A discussão geral a respeito da transição SU(4)-SU(2) é apresentada para a geometria de conexão lateral dos PQs, figura 2.1 (b) [54], discutida na seção 2.4. A física deste sistema é similar a encontrada usando um único PQ de nanotubo de carbono, onde o grau de liberdade extra, além do spin, é oriundo do número quântico que define a quiralidade presente no nanotubo de grafeno [55, 56]. Se por outro lado, as duas configurações geométricas ilustradas nos painéis (a) e (b) da figura 2.1 apresentam resultados idênticos do ponto de vista da transição SU(4)-SU(2) e sua física relacionada, por outro a primazia desempenhada pela geometria nas propriedades de transporte eletrônico do sistema é um ponto chave para compreender as propriedades do filtro de spin no que se refere à condutância, as quais serão estudadas na seção 2.5. Finalmente, terminamos o capítulo com as conclusões do estudo deste sistema. 


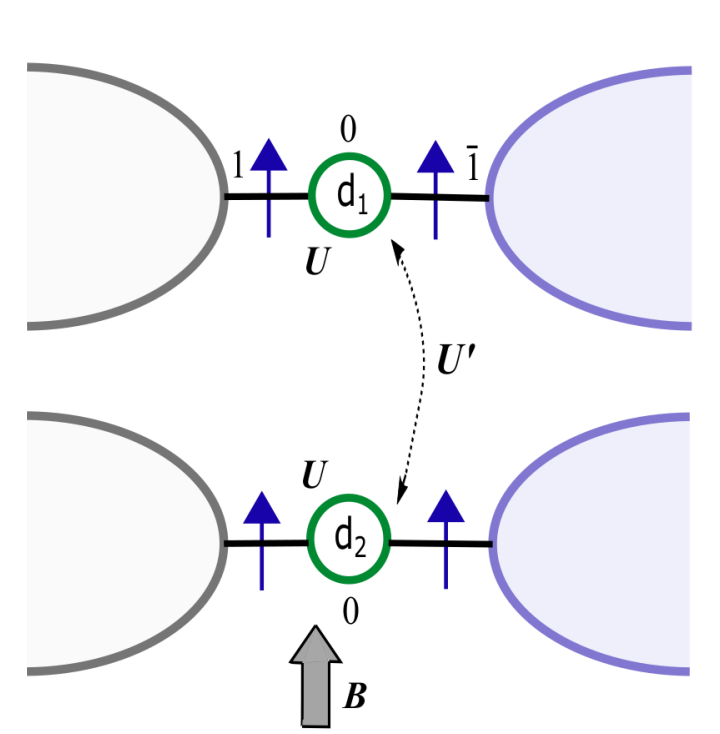

(a)

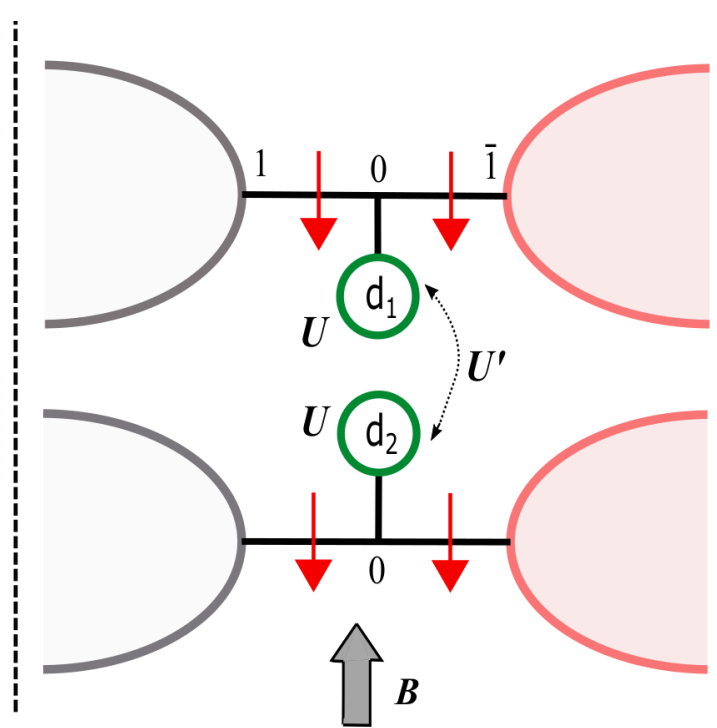

(b)

Figura 2.1: Sistema de dois PQs capacitivamente acoplados na presença de um campo magnético externo, que age somente sobre os PQs. $U^{\prime}(U)$ é a repulsão Coulombiana interponto (intraponto) os quais estão conectados a dois contatos por elementos de matriz $V$, não mostrados na figura. $\mathrm{O}$ sistema apresenta duas geometrias, na primeira com o PQ embebido nos contatos (a) e na segunda conectado lateralmente (b). Como será mostrado na seção 2.5 , um pequeno campo magnético pode polarizar o spin da corrente transmitida através dos PQs, com polarização paralela ao campo para os PQs embebidos (a) e antiparalela para o caso de conexão lateral (b), como ilustrado esquematicamente.

\section{2}

\section{Descrição do sistema}

O sistema analisado é composto por dois PQs paralelos, cada um conectado a um canal independente como esboçado na figura 2.1. Além da interação Coulombiana intra-PQ $U$, os PQs estão acoplados capacitivamente por uma interação Coulombiana inter-PQ $U^{\prime}$ e sob a influência de um campo magnético externo que produz o desdobramento Zeeman $B$.

O sistema será descrito por uma extensão do modelo de uma impureza de Anderson (MIA) $[7,57]$, apropriado para o estudo de duas impurezas, dado por,

$$
\begin{aligned}
H= & \sum_{j, \boldsymbol{k}_{j}, \sigma} \epsilon_{\boldsymbol{k}_{j}} c_{\boldsymbol{k}_{j} \sigma}^{\dagger} c_{\boldsymbol{k}_{j} \sigma}+\sum_{j, \sigma}\left(\left(\epsilon_{0}-\sigma B\right) n_{j \sigma}+\frac{U}{2} n_{j \sigma} n_{j \bar{\sigma}}\right)+ \\
& U^{\prime} \sum_{\sigma, \sigma^{\prime}} n_{1 \sigma} n_{2 \sigma^{\prime}}+\sum_{j, \boldsymbol{k}_{j}, \sigma} V_{\boldsymbol{k}_{j}}\left(d_{j \sigma} c_{\boldsymbol{k}_{j} \sigma}^{\dagger}+d_{j \sigma}^{\dagger} c_{\boldsymbol{k}_{j} \sigma}\right) .
\end{aligned}
$$

O primeiro termo descreve o mar de Fermi não interagente com dispersão $\epsilon_{\boldsymbol{k}_{j}}$, 
onde $j=1,2$ rotula os PQs e os respectivos contatos (depois de uma transformação simétrica/anti-simétrica entre os contatos da direita e esquerda). $c_{\boldsymbol{k}_{j} \sigma}^{\dagger}\left(c_{\boldsymbol{k}_{j} \sigma}\right)$ é o operador de criação (aniquilação) de um elétron com momento $|\boldsymbol{k}|=k$ e spin $\sigma$ no contato $j$. O segundo termo descreve cada PQ, (impureza magnética do MIA) com potencial de porta, ou elemento diagonal da impureza, $\epsilon_{0}$, o qual supomos o mesmo aplicado em cada PQ $j$. Também atua no PQ um campo magnético externo, que origina um desdobramento Zeeman $B$ entre os estados locais em função do spin. A repulsão Coulombiana intra-PQ está caracterizada pelo parâmetro $U$ e os números de ocupação são definidos como $n_{j \sigma} \equiv d_{j \sigma}^{\dagger} d_{j \sigma}$, onde $d_{j \sigma}^{\dagger}\left(d_{j \sigma}\right)$ cria (aniquila) um elétron com spin $\sigma$ no PQ $j$. O terceiro termo descreve a interação Coulombiana $U^{\prime}$ entre os elétrons situados em diferentes PQ com spins $\sigma$ e $\sigma^{\prime}$. Finalmente, o quarto e último termo descreve a hibridização entre cada PQ e o correspondente contato através do elemento de matriz $V_{\boldsymbol{k}_{j}}$. Como é usual, tomamos $V_{\boldsymbol{k}_{j}}=V$ independente de $\boldsymbol{k}$ para todo valor $\boldsymbol{k}$. A menos de mencionado o contrário, por simplicidade, consideramos os PQ idênticos, o que supõe $n_{1 \sigma}=n_{2 \sigma}$. A partir deste momento abandonaremos o subscrito $j$ que indexa cada PQ no que se refere à ocupação e redefiniremos $n_{\sigma}$ como a ocupação para cada spin $\sigma$ nos dois PQs, ou seja, $n_{\sigma}=n_{1 \sigma}+n_{2 \sigma}$.

$\mathrm{Na}$ ausência de campo magnético externo, o sistema possui simetria $\mathrm{SU}(4)$, rigorosamente, quando os potenciais de porta $\epsilon_{0}$ e os elementos de matriz de hibridização $V$ são independentes de $j$ além de $U^{\prime}=U$. Em especial, assumimos $U$ e $U^{\prime}$ infinitos com o objetivo de simplificar significativamente os cálculos numéricos, o que restringe as ocupações nos PQs a zero ou um. Contudo, o caso com $U^{\prime}$ finito é considerado no contexto da MFSBA a fim de mostrar que, na região apropriada dos parâmetros, as propriedades físicas do sistema não dependem de um valor particular da interação Coulombiana inter-PQ.

\section{3}

\section{Tratamento do sistema com duas impurezas pelo formalismo de opera- dores de projeção}

Nesta seção discutiremos a extensão do formalismo dos operadores de projeção (POA) para tratar o modelo da impureza de Anderson com duas impurezas, técnica utilizada para descrever as propriedades do sistema de DPQ acoplados capacitivamente. Apesar da análise efetuada com os POA ter sido realizada no limite da repulsões Coulombianas infinitas, i.e., $U=U^{\prime} \rightarrow \infty$, o caso com $U^{\prime}$ finito é explicitamente tradado com a abordagem da MFSBA [12, 28, 29], o que consiste em uma situação mais realista. Entretanto, como 
mostraremos ao longo do capítulo, na região do espaço de parâmetros $\left|\epsilon_{0}\right|<U^{\prime}$, os resultados não dependem qualitativamente de um valor particular de $U^{\prime} / U$.

A extensão do POA para sistemas com duas impurezas é apresentada no apêndice A que acompanha esta tese.

Com a finalidade de encontrar a energia do estado fundamental, precisamos resolver autoconsistentemente as funções $f_{1}(\xi)$ e $f_{0}(\xi)$, que dadas por

$\mathrm{e}$

$$
f_{1}(\xi)=\sum_{\sigma} \int_{-\infty}^{\epsilon_{F}}\left\{\rho(\omega) \frac{2 V^{2}}{\xi+\omega-\epsilon_{0}+\sigma B-f_{0}(\xi+\omega)}\right\} d \omega
$$

$$
f_{0}(\xi)=\int_{\epsilon_{F}}^{\infty}\left\{\rho(\omega) \frac{V^{2}}{\xi-\omega-f_{1}(\xi-\omega)}\right\} d \omega .
$$

A correção à energia do estado fundamental, $\Delta E$, é obtida da mesma forma que para o caso de apenas uma impureza, através da equação

$$
\Delta E=f_{1}(\Delta E)
$$

onde $\Delta E$ é definido como

$$
\Delta E=E-2 \epsilon_{T} .
$$

A energia do estado fundamental é $E$ e o somatório das energias dos elétrons por baixo do nível de Fermi $\epsilon_{F}$ é

$$
\epsilon_{T}=2 \int_{-\infty}^{\epsilon_{F}} \omega \rho(\omega),
$$

com $\rho(\omega)$ sendo a densidade de estados do mar de Fermi para cada contato.

A figura 2.2 apresenta a solução autoconsistente da função $f_{1}(\xi)$, equação (2-2), para três valores diferentes do potencial de porta $\epsilon_{0}$.

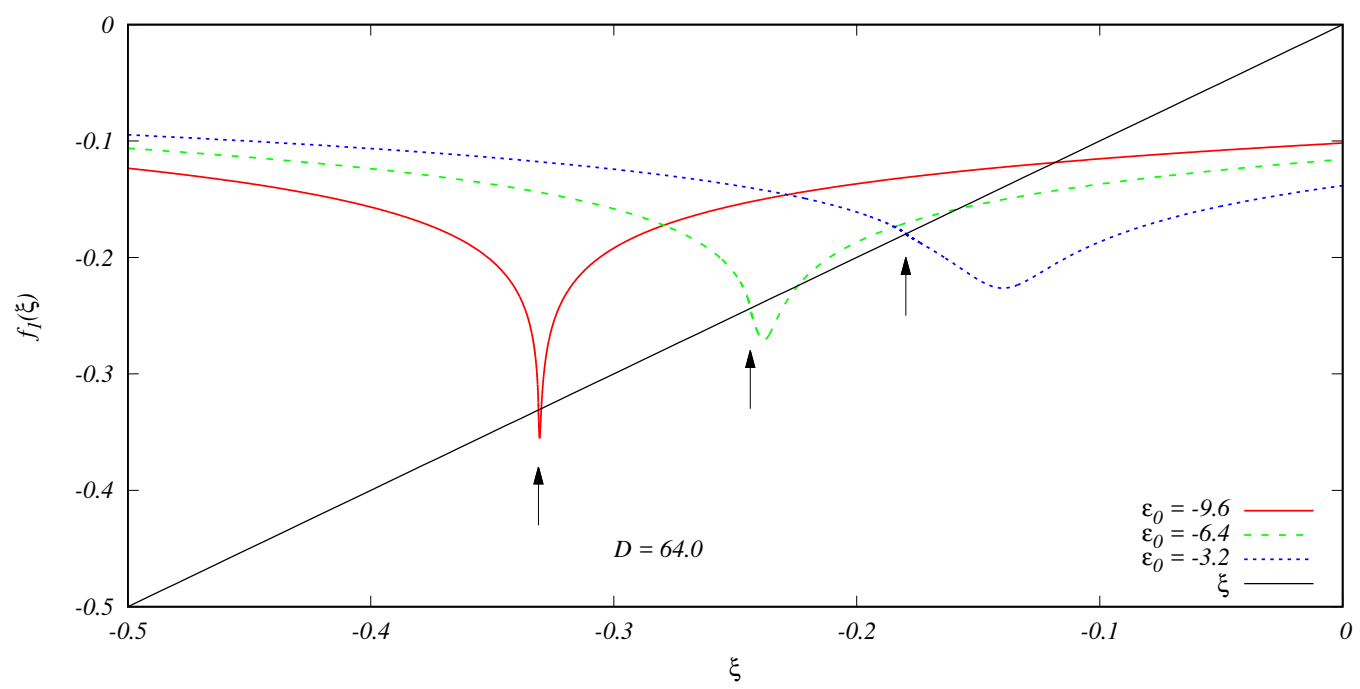

Figura 2.2: A função $f_{1}(\xi)$ resolvida autoconsistentemente para diferentes valores de $\epsilon_{0}$ com um campo magnético externo nulo. As soluções para a correção da energia do estado fundamental, $\Delta E$, obtidas pelos POA são indicadas pelas setas. Todas as grandezas estão expressas em unidades de $\Delta$. 


\section{4}

\section{Transição SU(4)-SU(2)}

Abordaremos agora o estudo da transição $\mathrm{SU}(4)-\mathrm{SU}(2)$ produzida por um campo magnético externo aplicado aos PQs do sistema em questão, onde a transição será caracterizada pelos números de ocupação nos PQs. Com este fim, $\left\langle n_{\uparrow}\right\rangle$ e $\left\langle n_{\downarrow}\right\rangle$ em cada PQ é calculado como função do potencial de porta usando os métodos de POA e MFSBA. Os resultados são apresentados em unidades de $\Delta$, função de hibridização e largura dos estados localizados nos PQ, cuja expressão é $\Delta=\pi V^{2} \rho\left(\epsilon_{F}\right)$, onde $\rho\left(\epsilon_{F}\right)$ é a densidade de estados ao nível de Fermi. Adotamos um valor do desdobramento Zeeman $B=3.2 \times 10^{-3} \Delta$, que como mostraremos mais adiante para valores típicos de GaAs podem corresponder a valores de campos magnéticos externos próximos a 0.1 Tesla.

A ocupação eletrônica para cada spin nos PQs $\left\langle n_{\sigma}\right\rangle$, como função do potencial de porta $\epsilon_{0}$ é calculada usando POA e MFSBA, como mostrado nas figuras 2.3 (a) e 2.3 (b). Se no caso do formalismo dos bósons escravos a ocupação é obtida integrando a densidade de estados nos PQs obtida através das funções de Green, no dos POA tomamos a derivada de $\Delta E$ com respeito a $\epsilon_{0}$ para calcular $\left\langle n_{\sigma}\right\rangle$. Na figura 2.3 (a), também mostramos a comparação do número de ocupação total, $\left\langle n_{t}\right\rangle=\left\langle n_{\uparrow}\right\rangle+\left\langle n_{\downarrow}\right\rangle$, para ambos os formalismos. Os resultados para $B=3.2 \times 10^{-3} \Delta$ na figura 2.3 (a) mostram uma concordância semiquantitativa entre as técnicas utilizadas, POA (símbolos) e MFSBA (curvas sólidas).

Na figura 2.3 (b), apresentamos os $\left\langle n_{\sigma}\right\rangle$ obtidos com POA e MFSBA para quatro valores de campo magnético externo, os quais percorrem quatro ordens de grandeza. Supomos que o acoplamento entre cada PQ e o respectivo contato seja $V=8.0 \Delta$, enquanto a semilargura dos contatos $D=64.0 \Delta$. Inspecionando-a, vemos que enquanto $\epsilon_{0}$ está próximo ao nível de Fermi e, portanto, os PQs estão no regime de flutuação de carga, os dois valores menores para o desdobramento Zeeman $\left(B=3.2 \times 10^{-4} \Delta\right.$ e $\left.3.2 \times 10^{-3} \Delta\right)$ são insuficientes para minimizar a energia do sistema, polarizando os PQs, quando comparados ao ganho de energia provido pela formação do singleto do efeito Kondo SU(4) do estado fundamental. Assim sendo, o campo magnético não é uma grandeza relevante neste regime, já que o estado fundamental não reflete a simetria $\mathrm{SU}(2)$ quebrada induzida pelo campo [48]. Contudo, a medida que o potencial de porta é reduzido, a temperatura Kondo $T_{K}^{S U(4)}$ decresce exponencialmente e eventualmente torna-se menor que $B$, quando uma mudança súbita no comportamento dos números de ocupação nos PQs $\left\langle n_{\sigma}\right\rangle$ é observada. $\left\langle n_{\downarrow}\right\rangle$ atinge um máximo e sofre uma queda acentuada, tendendo a zero conforme $\epsilon_{0}$ é reduzido, enquanto $\left\langle n_{\uparrow}\right\rangle$ continua crescendo 
Capítulo 2. Transição SU(4)-SU(2) e propriedades de filtro de spin de um nanossistema de dois pontos quânticos
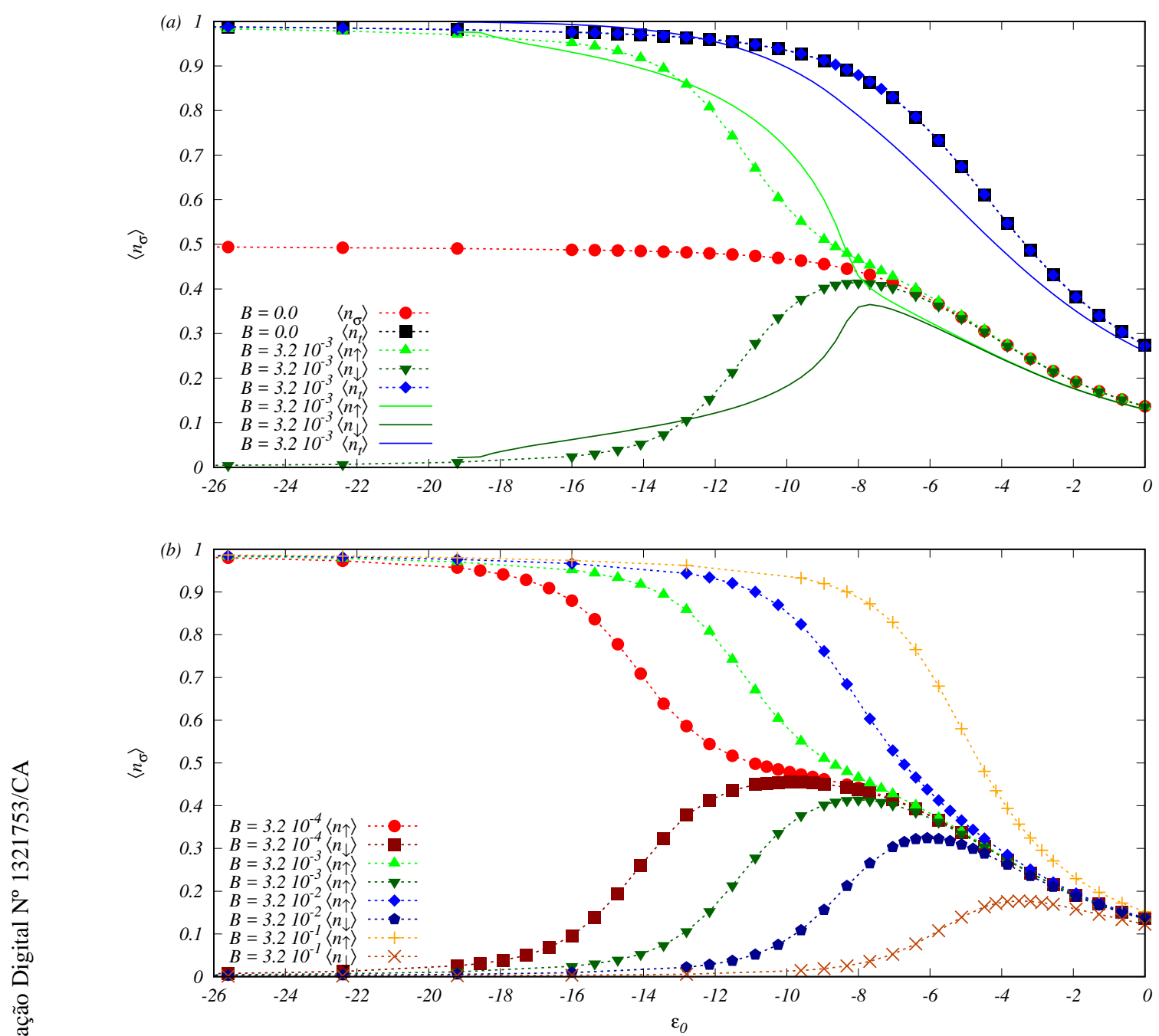

Figura 2.3: Números de ocupação eletrônica nos PQs $\left\langle n_{\sigma}\right\rangle$ e $\left\langle n_{t}\right\rangle=\left\langle n_{\uparrow}\right\rangle+\left\langle n_{\downarrow}\right\rangle$ como função do potencial de porta $\epsilon_{0}$ [12]. (a) comparação dos resultados de $\left\langle n_{\uparrow}\right\rangle,\left\langle n_{\downarrow}\right\rangle$, e $\left\langle n_{t}\right\rangle$ obtidos com o POA (símbolos) e MFSBA (curvas sólidas), para $B=3.2 \times 10^{-3} \Delta$. Observe que os resultados para POA e bósons escravos concordam semiquatitativamente. Também no mesmo painel a ocupação na ausência de campo magnético externo. (b) resultados dos POA $\left\langle n_{\sigma}\right\rangle$ para quatro valores de campo magnético externo. Todos os resultados em ambos os painéis são para $D=64.0 \Delta$ e são apresentados em unidades de $\Delta=1$.

e eventualmente saturando em $\left\langle n_{t}\right\rangle=\left\langle n_{\uparrow}\right\rangle=1$, refletindo o fato de $B$ ter sobrepujado $T_{K}^{S U(4)}$. Por outro lado, para os dois maiores valores de $B$, $B=3.2 \times 10^{-2} \Delta$ e $3.2 \times 10^{-1} \Delta$, a polarização começa a ocorrer para valores consideravelmente maiores de $\epsilon_{0}$, pois uma pequena redução de $\epsilon_{0}$ é suficiente para $T_{K}^{S U(4)} \lesssim B$. Todavia, deve ficar claro que esta discussão não implica que $B$ deva ser comparado irrestritamente à $T_{K}^{S U(4)}$ a campo nulo, devido ao campo magnético suprimir a temperatura Kondo como na figura 2.8 [13, 14].

O ponto de inflexão da curva $\left\langle n_{\downarrow}\right\rangle\left(\epsilon_{0}\right)$, ou em outras palavras, o máximo 
Capítulo 2. Transição SU(4)-SU(2) e propriedades de filtro de spin de um nanossistema de dois pontos quânticos

que ocorre quando $d\left\langle n_{\downarrow}\right\rangle / d \epsilon_{0}=0$, será utilizado para caracterizar a transição $\mathrm{SU}(4)-\mathrm{SU}(2)$, por ser um ponto característico da curva. Os resultados da figura 2.3 indicam que $\epsilon_{0}^{\max }$, o valor do potencial de porta em que ocorre o máximo de $\left\langle n_{\downarrow}\right\rangle$, depende fortemente de $B$, como esperado. A separação entre $\left\langle n_{\uparrow}\right\rangle$ e $\left\langle n_{\downarrow}\right\rangle$ ocorre para valores de $\epsilon_{0}^{\max }$ mais próximos do nível de Fermi $\epsilon_{F}$ quando os valores de $B$ são maiores.

Neste momento é interessante mencionar que os resultados qualitativos para os números de ocupação não dependem se tomamos $U^{\prime} \rightarrow \infty$. Como os cálculos usando o MFSBA não possui a mesma dificuldade, seja analítica ou numérica, que o POA, não estamos restritos ao caso $U=U^{\prime}$, como mostramos para $\left\langle n_{\sigma}\right\rangle$ como função de $\epsilon_{0}$ na figura 2.4 , com $U^{\prime}=64.0 \Delta$ e $U \rightarrow \infty$. Assim, podemos mostrar que os resultados apresentados nesta figura, figura 2.4, concordam qualitativamente com os de $U=U^{\prime} \rightarrow \infty$. Apesar de nesta situação o Hamiltoniano não possuir explicitamente uma simetria SU(4), não apenas devido à presença do campo magnético mas também por causa de $U^{\prime} \neq U$, o estado fundamental do sistema de dois PQs apresenta essa simetria até $\epsilon_{0} \approx-7.0 \Delta$ como uma propriedade emergente [47], logo, a transição SU(4)$\mathrm{SU}(2)$ pode ocorrer para valores menores de $\epsilon_{0}$ (compare com a figura 2.3).

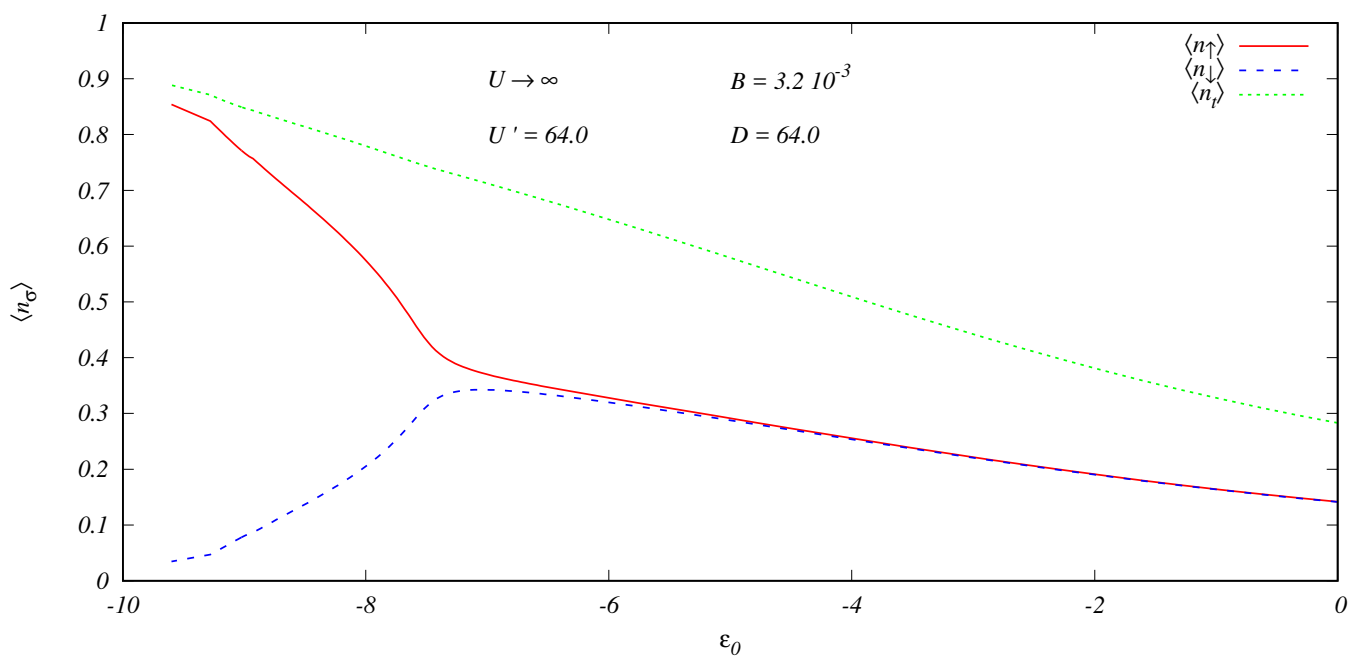

Figura 2.4: Resultados do formalismo de MFSBA, em unidades de $\Delta=1$, para $\left\langle n_{\uparrow}\right\rangle$ (linha contínua vermelha), $\left\langle n_{\downarrow}\right\rangle$ (curva azul tracejada), and $\left\langle n_{t}\right\rangle$ (curva verde pontilhada), como função do potencial de porta $\epsilon_{0}$ em um sistema de dois PQs acoplados capacitivamente, para $D=64.0 \Delta, U \rightarrow \infty, U^{\prime}=64.0 \Delta$ e $B=3.2 \times 10^{-3} \Delta$ [12]. Observe que os resultados são qualitativamente os mesmos que o obtido para $U=U^{\prime} \rightarrow \infty$, veja a figura 2.3.

De acordo com a literatura, a quebra da simetria SU(4), na presença de um campo magnético, só será claramente observada quando $B \approx T_{K}^{S U(4)}[13]$. A fim de elucidar este ponto, na figura 2.5 apresentamos um gráfico semilog com 
os resultados do POA para diferentes valores de $B_{\text {max }}$, em escala logarítmica, e os valores de $\epsilon_{0}^{\max }$ correspondentes no eixo horizontal. No gráfico principal desta figura, temos o resultados para a semilargura de banda, $D$, igual a $D=64.0 \Delta$ (círculos vermelhos), com um par de resultados extras no gráfico inserido, para $D=44.4 \Delta$ (quadrados verdes) e $D=16.0 \Delta$ (triângulos azuis), lembrando ao leitor que os resultados estão em unidades de energia de $\Delta=1$. Os resultados do painel principal e do inserido mostram claramente uma dependência exponencial de $B_{\max }$ em $\epsilon_{0}^{\max }$, onde utilizamos o algoritmo dos mínimos quadrados para realizar o ajuste da expressão

$$
B_{\max }=D \exp \left(a \epsilon_{0}^{\max }\right)
$$

traçado usando linhas contínuas. O valor de campo $B_{\text {max }}$ é uma escala relevante de energia que controla a transição $\mathrm{SU}(4)-\mathrm{SU}(2)$, que ocorre em $\epsilon_{0}^{\max }$ de acordo com nossa definição. Esta escala de energia tem um comportamento universal no regime Kondo, como descrito pela equação (2-7), bem como estendendo-se dentro da região de flutuação de carga, apesar de perder seu caráter universal na vizinhança do nível de Fermi, ilustrado pelos pontos mais próximos a ele - os quais não coincidem mais com a reta ajustada no painel principal da figura 2.5. A eventual perda de universalidade já era esperada, posto que o comportamento universal é tipicamente restrito ao regime Kondo, como no caso da temperatura Kondo. De qualquer modo, é importante enfatizar que para valores suficientemente grandes de $B,\left\langle n_{\downarrow}\right\rangle$ alcança um máximo mesmo dentro da região de flutuação de carga. Isto permite definir $B_{\max }$ como a escala de energia que governa a transição $\mathrm{SU}(4)-\mathrm{SU}(2)$ também neste regime.

Os resultados no gráfico menor inserido na figura 2.5 para três valores da semilargura de banda $D$ (tomando $\Delta$, nossa unidade de energia) claramente mostram que o parâmetro $a \sim 1.23$ (ver equação (2-7)) é independente de $D$, demonstrando o comportamento universal do campo magnético como escala que caracteriza a transição SU(4)-SU(2). Finalmente, o ajuste (linha contínua) também nos informa que a escola da semilargura de banda $D$ como pré-fator é correta, já que o mesmo é recuperado com boa acurácia numérica no ajuste para os valores de $D$ usados no cálculo com POA.

No mesmo gráfico, apresentamos no eixo vertical da direita também em escala logarítmica as temperaturas Kondo $T_{K}^{S U(2)}$ e $T_{K}^{S U(4)}$, linha tracejada e pontilhada, respectivamente, obtidas através da expressão [58]

$$
T_{K}^{S U(N)}=D \exp \left(\pi \epsilon_{0} / N\right)
$$

que é conseguida através de uma função de onda variacional com $U \rightarrow \infty$ para o estado fundamental do sistema [59], a qual coincide bem com a 


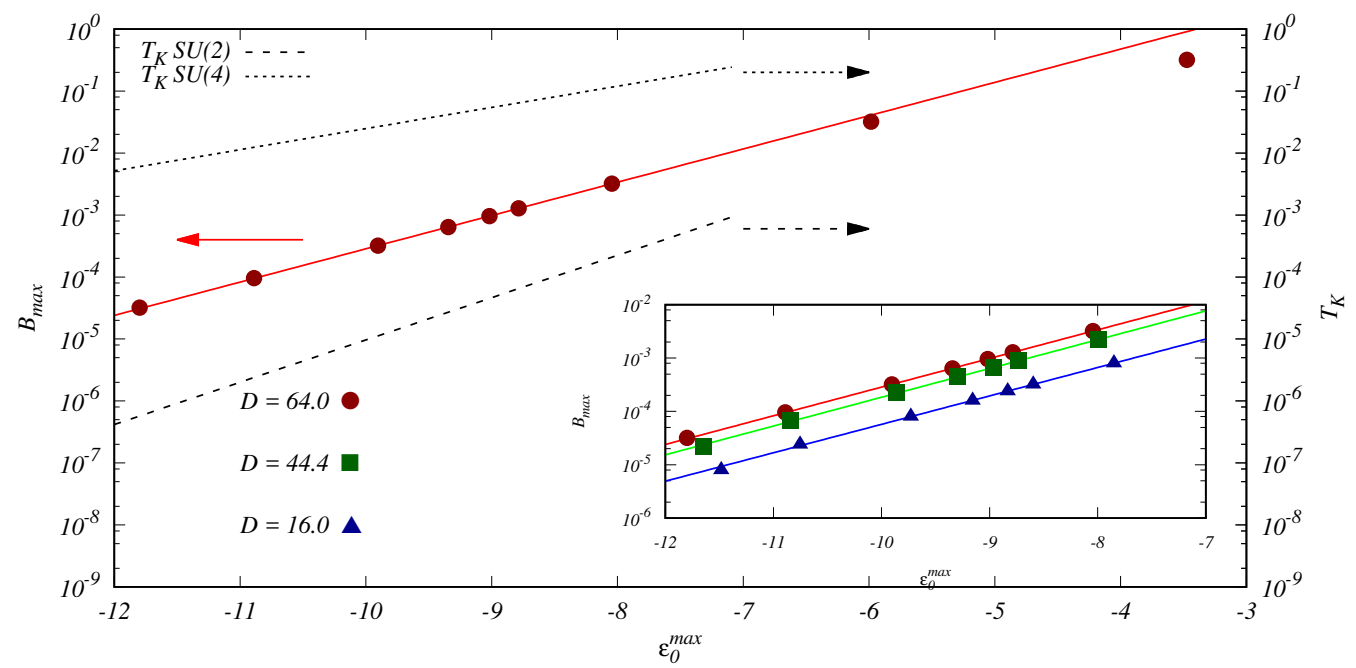

Figura 2.5: Gráfico semilog de $B_{\max }=f\left(\epsilon_{0}^{\max }\right)$ obtido usando POA para três valores diferentes de semilargura de banda $D$. Os resultados estão apresentados em unidades de $\Delta=1$. No gráfico maior temos a comparação do resultado com $D=64 \Delta$ com as curvas para as diferentes simetrias da temperatura Kondo, $\mathrm{SU}(4)$ na curva pontilhada e $\mathrm{SU}(2)$ na tracejada, que foram obtidas a partir da equação (2-8). Este resultado mostra que $B_{\max }$ tem uma escala de energia intermediária entre as $T_{K}^{S U(4)}$ e $T_{K}^{S U(2)}$. Apresentamos no gráfico menor inserido os resultados para $D=44.4 \Delta$ e $D=16 \Delta$, além do resultado com $D=64 \Delta$. Nele verificamos a ausência de $D$ na dependência exponencial do fator $a$ (ver equação (2-7)), que configura o caráter universal da transição SU(4)-SU(2) comandada pelo campo magnético.

solução de MFSBA no mesmo limite [58]. Para facilitar a comparação com a dependência exponencial de $B_{\max }\left(\epsilon_{0}^{\max }\right)$, equação (2-7), apresentamos também na figura 2.5 as temperaturas Kondo $T_{K}^{S U(2)}$ e $T_{K}^{S U(4)}$, equação (2-8). Estas duas temperaturas são esboçadas apenas para valores de $\epsilon_{0}<-7 \Delta$, que aproximadamente define uma região do regime Kondo, para enfatizar que as expressões acima não são válidas no regime de flutuação de carga.

É importante mencionar que o fator exponencial do campo magnético na equação (2-7), $a \sim 1.23$, tem um valor intermediário entre os estados Kondo $T_{K}^{S U(4)}$ e $T_{K}^{S U(2)}$ (ver equação (2-8)): $\pi / 4<a<\pi / 2$. Uma simples inspeção da figura 2.5 mostra que o valor de $B_{\max }$ está entre uma e duas ordens de grandeza menor que $T_{K}^{S U(4)}$ e igualmente maior que $T_{K}^{S U(2)}$, com uma diferença mais acentuada para valores menores de $\epsilon_{0}$, cada vez mais dentro do regime Kondo. Como o valor do fator exponencial que controla $B_{\max }$ está entre os correspondentes para as temperaturas Kondo $T_{K}^{S U(4)}$ e $T_{K}^{S U(2)}$, é possível operar o sistema de DPQ em uma região de alta polarização de spin $\left(\left\langle n_{\uparrow}\right\rangle \gg\left\langle n_{\downarrow}\right\rangle\right)$ para um valor pequeno do campo. Este fato tem importantes consequências 
Capítulo 2. Transição SU(4)-SU(2) e propriedades de filtro de spin de um nanossistema de dois pontos quânticos

para a performance de um filtro de spin, como será discutido na seção 2.5.

Para caracterizar apropriadamente a transição SU(4)-SU(2), é interessante investigar o oposto ao realizado até o momento, ou seja, ao invés de fixar um campo magnético externo e analisar como $\left\langle n_{\sigma}\right\rangle$ depende de $\epsilon_{0}$, nós estudamos a variação de $\left\langle n_{\sigma}\right\rangle$ como função do campo magnético para um $\epsilon_{0}$ fixo. A ideia principal é utilizar $\epsilon_{0}$ para colocar o sistema, a campo nulo, bem dentro da região Kondo de simetria SU(4) e o outro valor próximo a região de flutuação de carga, e analisar como a aplicação de um campo magnético externo nos PQs modificam as propriedades do sistema. Nós estudamos o número de ocupação para cada spin $\left\langle n_{\uparrow}\right\rangle$ e $\left\langle n_{\downarrow}\right\rangle$ usando POA. O resultado é apresentado na figura 2.6, onde as linhas contínuas indicam $\left\langle n_{\uparrow}\right\rangle$ e as tracejadas $\left\langle n_{\downarrow}\right\rangle$ para quatro diferentes valores do potencial de porta $\epsilon_{0}: \epsilon_{0}=-19.2 \Delta$ (triângulos azuis) coloca o sistema profundamente dentro da região Kondo de simetria SU(4), $\epsilon_{0}=-9.6 \Delta$ (círculos vermelhos) situa o sistema próximo ao regime de flutuação de carga, enquanto que para $\epsilon_{0}=-14.4 \Delta$ (quadrados verdes) o sistema se encontra no meio do caminho entre estas duas situações. Este conjunto de três dados foram obtidos para uma semilargura de banda $D=64.0 \Delta$ e adicionamos um quarto resultado (triângulos invertidos magenta) com $\epsilon_{0}=-11.1 \Delta$ com $D$ menor, $D=44.4 \Delta$, para analisar os efeitos em $D$ nos resultados obtidos. Os resultados na figura 2.6 indicam que, próximo da fronteira do regime de flutuação de carga, $\epsilon_{0}=-9.6 \Delta$ e $-11.1 \Delta$, e mesmo dentro da região do regime Kondo SU(4), $\epsilon_{0}=-14.4 \Delta$, a polarização de spin, medida como $\left\langle n_{\uparrow}\right\rangle-\left\langle n_{\downarrow}\right\rangle$, é gradualmente aumentada em resposta ao acréscimo, a partir de zero, do campo magnético (veja as curvas com os círculos, triângulos invertidos e quadrados). Este comportamento pode ser atribuído aos valores maiores de $T_{K}^{S U(4)}$ para $\epsilon_{0}$ próximo ao nível de Fermi (ver curva pontilhada na figura 2.5), que necessitará de um campo maior para forçar o sistema a passar do regime de simetria $\mathrm{SU}(4)$ para $\mathrm{SU}(2)$. Isto é especialmente evidente para os resultados com $\epsilon_{0}=-9.6 \Delta$ (círculos vermelhos), que possui a maior $T_{K}^{S U(4)}$ dentre os resultados apresentados, onde um campo maior é requerido para gerar uma polarização de spin satisfatória. Logo, espera-se que o sistema exija um campo magnético muito pequeno para transitar do regime $\mathrm{SU}(4)$ para o regime de Kondo orbital $\mathrm{SU}(2)$, para um $T_{K}^{S U(4)}$ muito menor. Esta situação é exatamente a observada para $\epsilon_{0}=-19.2 \Delta$ (triângulos azuis), onde $T_{K}^{S U(4)}$ é muito menor (ver figura 2.5) e o sistema responde de forma muito mais abrupta ao campo magnético. Na verdade, mesmo os resultados para $\epsilon_{0}=-14.4 \Delta$ (quadrados verdes), onde $T_{K}^{S U(4)}$ não é tão pequena, mostra que um pequeno campo magnético externo de aproximadamente 0.1 Tesla ou $B \approx 0.0022 \Delta$, se tomarmos o fator giromagnético para GaAs, é suficiente para obter uma polarização de spin considerável, como 
Capítulo 2. Transição SU(4)-SU(2) e propriedades de filtro de spin de um nanossistema de dois pontos quânticos

ilustrado na figura 2.6.

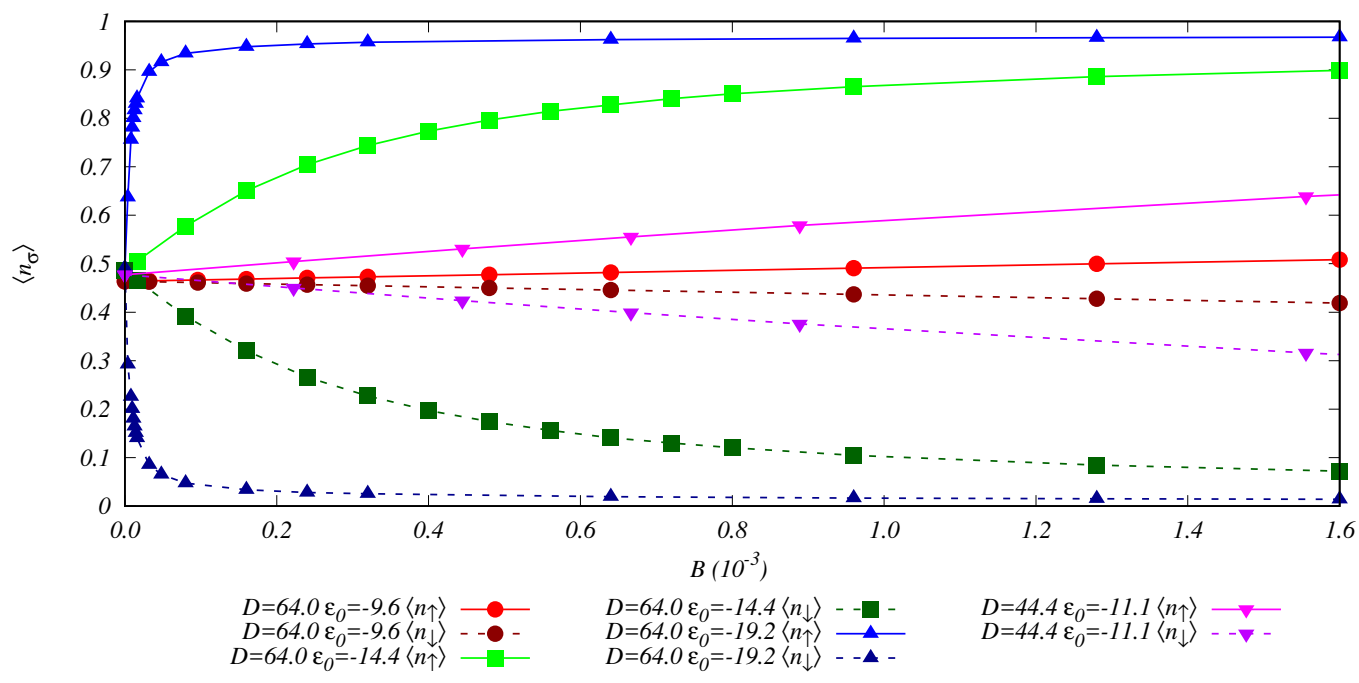

Figura 2.6: Resultados do POA para $\left\langle n_{\uparrow}\right\rangle$ (linhas sólidas) e $\left\langle n_{\downarrow}\right\rangle$ (linhas tracejadas), como função do desdobramento Zeeman $B$ para quatro valores de $\epsilon_{0}=-9.6 \Delta$ (círculos vermelhos), $-11.1 \Delta$ (triângulos invertidos magenta), $-14.4 \Delta$ (quadrados verdes) e $-19.2 \Delta$ (triângulos azuis). Note a polarização gradual do sistema quando no regime de flutuação de carga (círculos vermelhos) e a mudança abrupta, a campos pequenos, do Kondo SU(4) para SU(2) quando o sistema está dentro da região Kondo SU(4) a campo nulo (triângulos azuis). Todos os resultados apresentados em unidades de $\Delta=1$ e realizados para $D=64.0 \Delta$, exceto para $\epsilon_{0}=-11.1 \Delta$, que foi obtido com $D=44.4 \Delta$.

Os resultados na figura 2.6 já eram esperados. O que os torna mais relevantes é apresentado na figura 2.7, onde é mostrado que se os dados de $\left\langle n_{\uparrow}\right\rangle$ e $\left\langle n_{\downarrow}\right\rangle$ da figura 2.6 são apresentadosm como função de $B / T_{K}^{S U(4)}$, com $T_{K}^{S U(4)}$ obtido da equação (2-8), ao invés de apenas $B$, todas as curvas da figura 2.6, com os diferentes parâmetros, colapsam em apenas uma. Isto é válido até para os dados com $\epsilon_{0}=-11.1 \Delta$ (triângulos invertidos magenta), que possui um valor de $D$ diferente dos demais dados do gráfico. Esta universalidade mostra que existe uma relação universal entre a polarização de spin e a razão ${ }^{B} / T_{K}^{S U(4)}$ quando um campo magnético externo é aplicado. É importante enfatizar que esta universalidade é obtida adotando a equação (2-8) para calcular $T_{K}^{S U(4)}$, o que fornece suporte adicional para a expressão de $T_{K}^{S U(4)}$ da equação (2-8) no limite $U \rightarrow \infty$.

Na figura 2.8 reproduzimos no eixo da esquerda $\left\langle n_{\sigma}\right\rangle$ para $\epsilon_{0}=-11.1 \Delta \mathrm{e}$ $D=44.4 \Delta$, como função do desdobramento Zeeman $B$ (triângulos invertidos magenta), junto às temperaturas Kondo $T_{K}^{S U(4)}$ (linha pontilhada) e $T_{K}^{S U(2)}$ (linha tracejada), a campo magnético nulo, no eixo da direita e em escala logarítmica. Ressalta-se que as temperaturas Kondo aparecem como linhas 


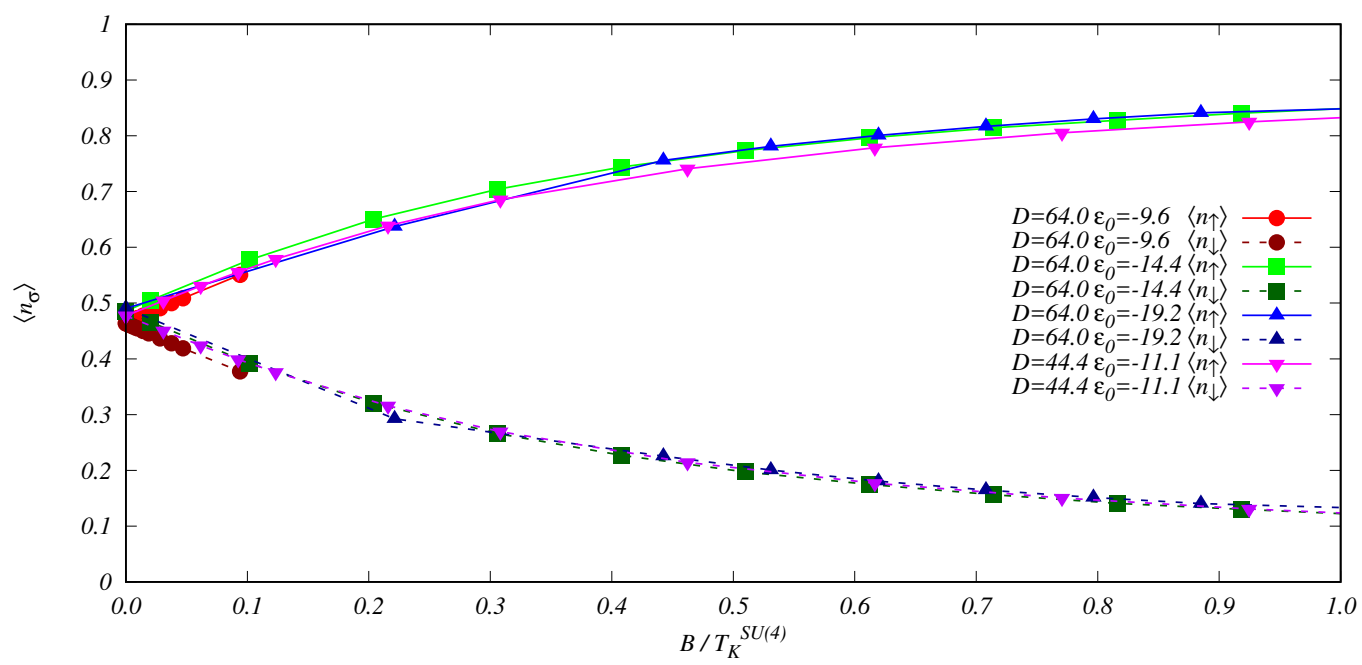

Figura 2.7: Mesmos dados da figura 2.6, mas agora traçados como função de $B / T_{K}^{S U(4)}$ ao invés de $B$.

horizontais, ou melhor, como constantes, pois usamos a equação (2-8). Como discutido anteriormente, na região da transição o sistema está em um estado fundamental Kondo que está passando da simetria SU(4) para SU(2). Uma estimativa da temperatura Kondo neste "estado de transição" e sua dependência com o desdobramento Zeeman pode ser obtida por um cálculo variacional, que interpola como função do campo as $T_{K}^{S U(4)}$ em $B=0$ e $T_{K}^{S U(2)}$ obtidas para $B \rightarrow \infty$ [14]. Esta temperatura Kondo interpolada, denotada como $T_{K}^{V a r}$, apresenta a forma

$$
T_{K}^{V a r}=\left\{(D+2 B) D \exp \left[\frac{\pi\left(\epsilon_{0}-B\right)}{2 \Delta}\right]+B^{2}\right\}^{\frac{1}{2}}-B
$$

e é apresentada na figura 2.8 como uma curva sólida negra. Obviamente, ela inicia-se em $T_{K}^{S U(4)}$, decresce com $B$ e, para intervalos pequenos de variação do campo como na figura, permanece ao menos três ordens de grandeza acima da $T_{K}^{S U(2)}$. Além disso, para $B \approx 0.0022 \Delta$, correspondente a um campo magnético externo de aproximadamente 0.1 Tesla, $T_{K}^{V a r}$ é quase igual a $T_{K}^{S U(4)}$, que, para os valores das parâmetros admitidos, resulta de ser da ordem de $10 \mathrm{~K}$. Estes valores de campo e temperaturas são perfeitamente acessíveis do ponto de vista experimental para a operação dos sistema de dois PQs capacitivamente acoplados como um filtro de spin, assunto que discutiremos na próxima seção. 


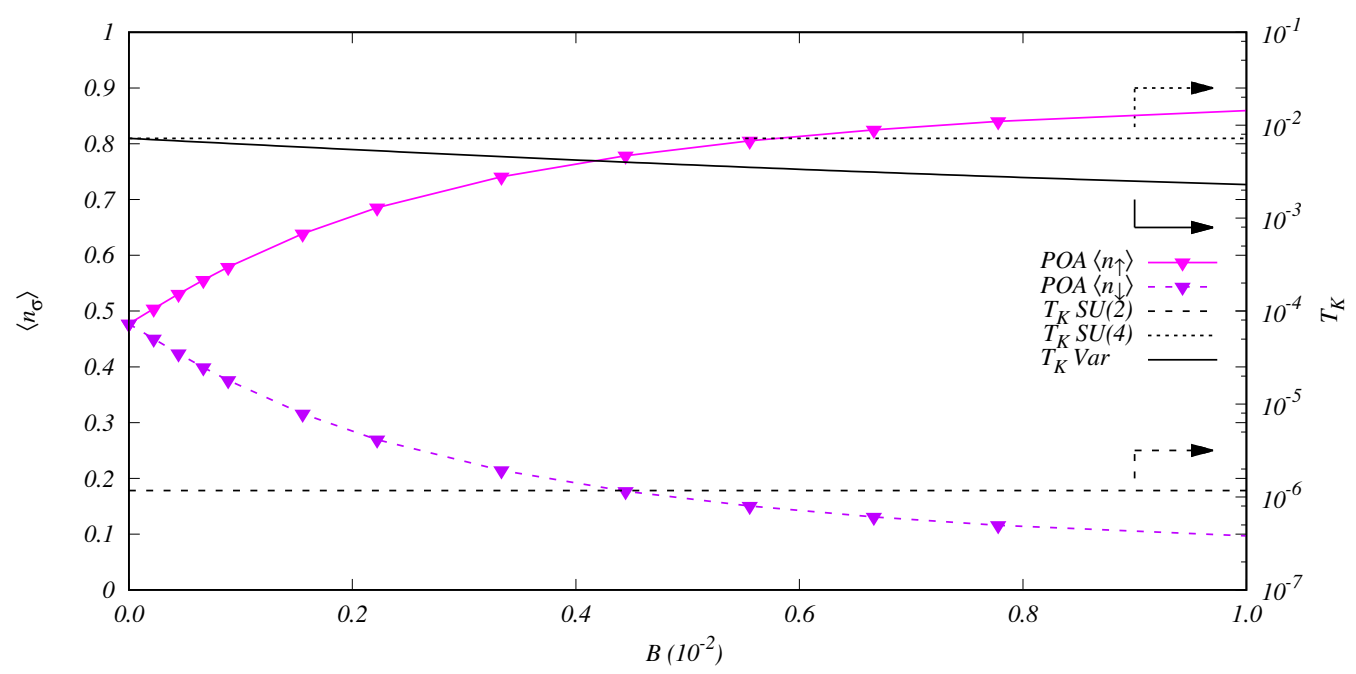

Figura 2.8: No eixo vertical da esquerda, $\left\langle n_{\sigma}\right\rangle$ como função de $B$ para $\epsilon_{0}=-11.11 \Delta$ (triângulos magenta), já no eixo vertical da direita, em escala logarítmica, as temperaturas Kondo $T_{K}^{S U(4)}$ (linha pontilhada) e $T_{K}^{S U(2)}$ (linha tracejada) com $B=0$ são representadas por linhas horizontais. A linha sólida negra é a temperatura Kondo para um "estado de transição", como função de $B$, obtido pelo método variacional da interpolação entre estados $\mathrm{SU}(4)$ e $\mathrm{SU}(2)[13,14]$. Resultados apresentados em unidades de energia com $\Delta=1$ para $\epsilon_{0}=-11.1 \Delta$ e $D=44.4 \Delta$.

\section{5}

\section{Filtro de Spin}

Excetuando-se o interesse da natureza intrínseca dos sistemas cujas características dependem da orientação do spin, também é importante verificar as possíveis aplicações porventura significativas que o sistema possa possuir sob controle adequado. As propriedades de filtro de spin de um PQ, ou estruturas de PQs, é um destes aspectos interessantes que tem sido estudado nos últimos anos [60-66]. A proposta de produzir correntes de spin polarizadas nos contatos através de um PQ é baseada na ideia de que o desdobramento Zeeman pode ter efeitos mais importantes no PQ do que nos contatos, criando, assim, um filtro de spin. Um filtro de spin é obtido quando o nível de spin para cima do PQ está localizado na janela de transporte, enquanto que o spin oposto fica fora dela. Isto requer um campo magnético grande, mesmo considerando o fator $g$ renormalizado para o PQ, e um fraco acoplamento do PQ com os contatos, resultando em estados localizados muito estreitos. Enquanto a primeira restrição impacta a realização experimental, introduzindo limitações a aplicabilidade do dispositivo, a segunda reduz significativamente a intensidade da corrente circulando por ele. Nenhuma das dificuldades descritas estão presentes no dispositivo proposto, já que como o sistema opera no regime 
Kondo, possui uma ressonância muito estreita com o spin polarizado, localizada na vizinhança do nível de Fermi e bem separada da outra polarização. Como o dispositivo impõe estar no regime Kondo, a temperatura do sistema deve ser inferior à temperatura Kondo, o que é uma limitação. Todavia, felizmente o desdobramento Zeeman requerido para distanciar $\left\langle n_{\uparrow}\right\rangle$ de $\left\langle n_{\downarrow}\right\rangle$ pode ser conseguida muito próximo da $T_{K}^{S U(4)}$, que embora abaixo deste valor é muito maior que a $T_{K}^{S U(2)}$, como já discutido.

A fim de esclarecer esses pontos e mostrar as potencialidades do filtro de spin constituído por um sistema de dois PQs acoplados capacitivamente, calculamos a corrente como função dos parâmetros relevantes, através de um dos PQs. Ela é dada por [67]:

$$
J=\frac{2 e}{h} \int_{-\infty}^{\infty} T(\epsilon)\left[f\left(\epsilon-\epsilon_{1}\right)-f\left(\epsilon-\epsilon_{-1}\right)\right] d \epsilon,
$$

onde $f(\epsilon)$ é a distribuição de Fermi-Dirac e $\epsilon_{1}$ e $\epsilon_{-1}$ são as energias de Fermi dos contatos, respectivamente. A matriz de transmissão $T(\epsilon)$, ao nível de Fermi, possui a forma [67]

$$
T\left(\epsilon_{F}\right)=4 \pi^{2} V_{e}^{4} \rho_{1}\left(\epsilon_{F}\right) \rho_{\overline{1}}\left(\epsilon_{F}\right)\left|G_{00}^{\sigma}\left(\epsilon_{F}\right)\right|^{2},
$$

onde $\rho_{1}\left(\epsilon_{F}\right)=\rho_{\overline{1}}\left(\epsilon_{F}\right)$ é a densidade de estados local no primeiro sítio dos contatos; ver rótulos na figura 2.1.

Para uma diferença de potencial infinitesimal - logo no regime linear, onde os processos inelásticos podem ser desprezados [68], e é possível supor $\epsilon_{1}=\epsilon_{-1}=\epsilon_{F}$ - obtemos da equação (2-10) uma expressão familiar para a condutância usando o formalismo de Keldysh [69], no contexto da técnica de bósons escravos na aproximação de campo médio,

$$
C^{\sigma}=\left(\frac{2 e^{2}}{h}\right) 4 \pi^{2} V_{e}^{4} \rho_{1}\left(\epsilon_{F}\right) \rho_{-1}\left(\epsilon_{F}\right)\left|G_{00}^{\sigma \sigma}\left(\epsilon_{F}\right)\right|^{2} .
$$

Para a configuração do PQ embebido, ver figura 2.1 (a), a função de Green calculada ao nível de Fermi, $G_{00}^{\sigma \sigma}\left(\epsilon_{F}\right)$, é dada por $G_{d d}^{\sigma \sigma}\left(\epsilon_{F}\right)$, que é a função de Green vestida no $\mathrm{PQ}$ com $V_{e}=V$. Ao passo que para o caso de conexão lateral dos PQs, ver figura 2.1 (b), $V_{e}$ é o elemento de matriz não diagonal na representação de ligações fortes dos contatos, i.e., $V_{e}=t$, e $G_{00}^{\sigma \sigma}\left(\epsilon_{F}\right)$ é

$$
G_{00}^{\sigma \sigma}\left(\epsilon_{F}\right)=g_{0}+g_{0}^{2} V^{2} G_{d d}^{\sigma \sigma}\left(\epsilon_{F}\right)
$$

onde $g_{0}$ corresponde a função de Geeen despida no primeiro sítio de uma cadeia linear semi-infinita.

Este cálculo é simples usando o método MFSBA, já que as funções 
de Green podem ser obtidas diretamente. Entretanto, na perspectiva do formalismo do POA a condutância tem que ser calculada a partir dos resultados prévios do número de ocupação nos PQs, usando a regra da soma de Friedel [7], que serão descritos no apêndice B.

Usando as propriedades de um líquido de Fermi a condutância pode ser escrita em termos do número de ocupação $\left\langle n_{\sigma}\right\rangle$, para o caso dos PQs embebidos, como

$$
C^{\sigma}\left(\frac{e^{2}}{h}\right)=\operatorname{sen}^{2}\left[\frac{\pi}{2}\left\langle n_{\sigma}\right\rangle\right] .
$$

Já para os PQs conectados lateralmente é possível relacionar $\left|G_{00}^{\sigma \sigma}\left(\epsilon_{F}\right)\right|^{2}$ com a ocupação eletrônica nos PQs através da equação (2-13). Com um raciocínio análogo ao empregado, a condutância resulta ser,

$$
C^{\sigma}\left(\frac{e^{2}}{h}\right)=1-\operatorname{sen}^{2}\left[\frac{\pi}{2}\left\langle n_{\sigma}\right\rangle\right]
$$

Os detalhes do cálculo das equações (2-14) e (2-15) são apresentados no apêndice B.

Usando as equações acima, mostramos na figura 2.9 (a) o resultado da condutância obtida para o caso dos PQs embebidos, sobre efeito de um campo magnético externo, como função do potencial de porta. Ao passo que na figura 2.9 (b) apresentamos os resultados para os PQs conectados lateralmente. Os dados adquiridos com o POA são representados como símbolos (triângulos) e o do MFSBA como as linhas contínuas e tracejadas.

Inspecionando a figura, permite-nos concluir que ambas as abordagens fornecem resultados qualitativamente equivalentes para as propriedades de transporte. Na região de $\epsilon_{0}<-12.0 \Delta$, para ambos os painéis, a condutância para um spin é quase $2 e^{2} / h$, ao passo que para o outro spin é próxima a zero, mostrando-se um resultado interessante, que mesmo com campos magnéticos relativamente baixos, inferiores a 0.1 Tesla para o caso do GaAs, o dispositivo de dois PQs opera como um filtro de spin muito eficiente. É importante destacar que, no caso dos PQs acoplados lateralmente, figura 2.9 (b), o papel do spin eletrônico é intercambiado, i.e., os elétrons transmitidos possuem spin para baixo (sentido oposto ao campo), enquanto que para os PQs embebidos possuem spin para cima (sentido paralelo ao campo). Para a configuração de conexão lateral dos PQs, figura 2.9 (a), quando o sistema está no regime Kondo, o elétron com spin para cima que flui através do sistema tem dois canais para se deslocar, um conectando os contatos diretamente enquanto o outro que visita o PQ. Como eles possuem fases opostas, a interferência destrutiva entre eles 

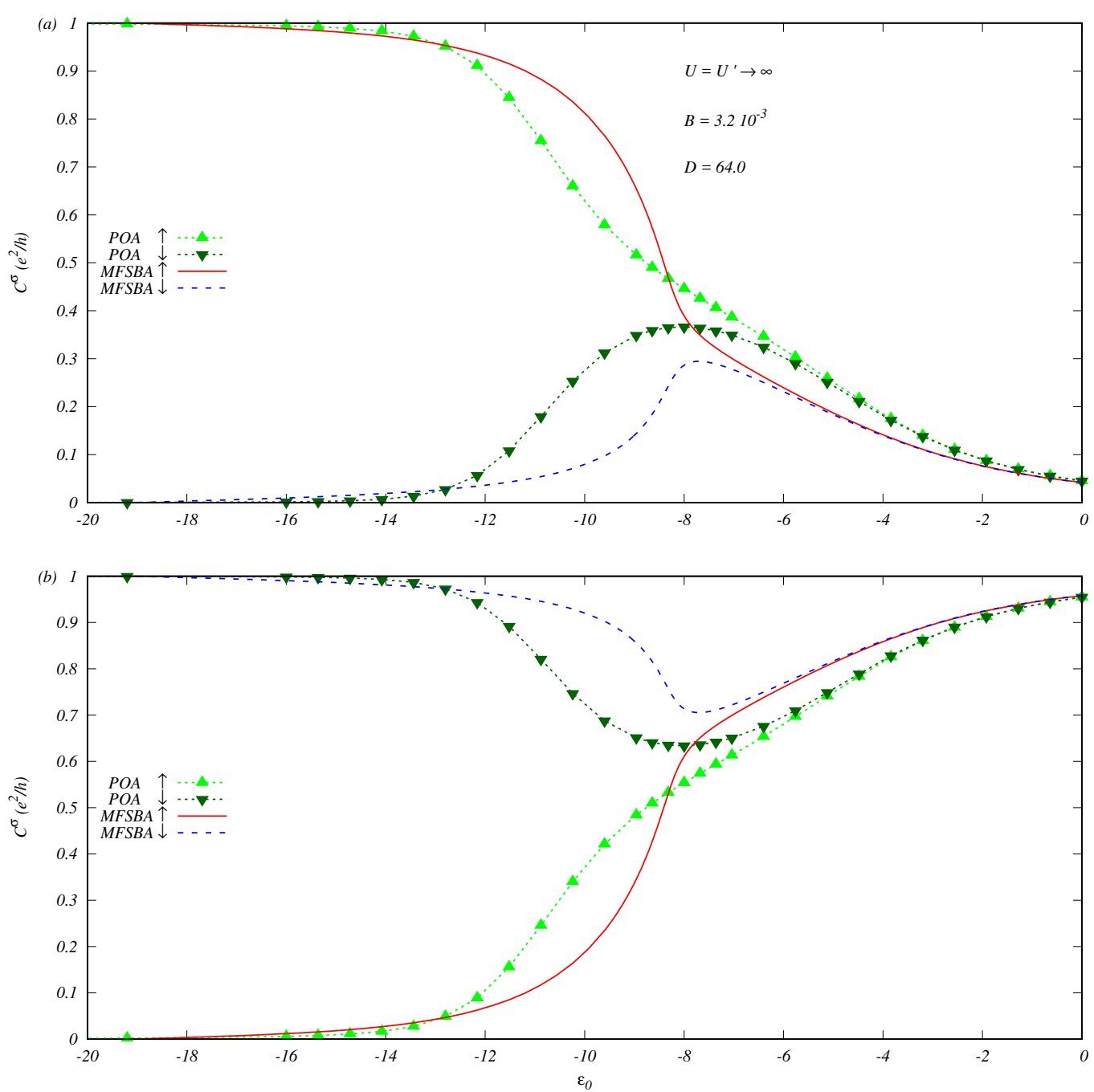

Figura 2.9: Condutância para cada spin, $C^{\sigma}$, como função do potencial de porta $\epsilon_{0}$, obtida por ambos os formalismos. Os resultados do POA são traçados como símbolos, já o do MFSBA como linhas. No painel (a) para o caso da geometria do PQ embebido e no (b) para o conectado lateralmente. Os parâmetros usados para cálculo são $D=64.0 \Delta, B=3.2 \times 10^{-3} \Delta$ e $U=U^{\prime} \rightarrow \infty$, em unidades de $\Delta=1$.

origina uma típica anti-ressonância Fano. Esta interferência destrutiva, sobre a polarização de spin, resulta no efeito oposto, com a polarização oposta a direção do campo, em comparação aos PQs embebidos. Neste caso, o elétron com spin para baixo é o único transmitido, enquanto que a condutância com spin para cima desaparece rapidamente ao diminuímos $\epsilon_{0}$, como visto na figura 2.9 (b). 


\section{6}

\section{Conclusões}

Neste capítulo, foi discutida a transição SU(4)-SU(2) produzida por um campo magnético externo, para o sistema de dois PQs acoplados capacitivamente e conectados a contatos metálicos. A transição produzida pelo desdobramento Zeeman foi caracterizada pelo máximo no $\left\langle n_{\downarrow}\right\rangle$ como função do potencial de porta, que ocorre em $\epsilon_{0}^{\max }$. Chamamos de $B_{\max }$ o valor do desdobramento Zeeman em que ocorre o máximo de $\left\langle n_{\downarrow}\right\rangle$. Sua dependência funcional, $B_{\max }=f\left(\epsilon_{0}^{\max }\right)$, define uma função universal $B_{\max }=D \exp \left(a \epsilon_{0}^{\max }\right)$, no regime Kondo, como apresentado com detalhes na figura 2.4. Esta universalidade é perdida na medida em que entra-se no regime de flutuação de carga, o mesmo que acontece com a temperatura Kondo. Todavia, é importante enfatizar que a ocorrência do máximo estende-se dentro do regime de flutuação de valência, o qual permite definir a escala de energia $B_{\max }$ como a magnitude que controla a transição SU(4)-SU(2) independentemente do regime do sistema.

Foi possível mostrar que na região da transição, para uma temperatura Kondo efetiva próxima da região com simetria $\mathrm{SU}(4)$, mas ainda dentro da região SU(2), a população eletrônica nos PQs é significativamente polarizada ao longo do campo magnético. Ademais, dependendo dos parâmetros do sistema, a polarização pode ser obtida mesmo para campos magnéticos pequenos, próximos a 0.1 Tesla para o caso do GaAs e a temperatura Kondo pode ser da ordem de alguns graus Kelvin. Com respeito à realização experimental, cabe mencionar que em comparação ao dispositivo similar proposto na referência [61], o apresentado aqui pode operar em campos consideravelmente menores.

Além disso, esta estrutura de dois PQs foi investigada usando dois formalismos diferentes, o projector operator approach (POA) e o mean-field slave bosons approximation (MFSBA), os quais foram capazes de descrever as propriedades mencionadas, fornecendo resultados qualitativamente equivalentes. Com este propósito, foi necessário estender o POA, originalmente derivado para o estudo de uma impureza de Anderson, para a análise de duas impurezas acopladas capacitivamente. Esta extensão fornece resultados praticamente exatos, ao menos nas referidas propriedades estáticas a temperatura zero

Conclui-se que este sistema, sobre influência de um campo magnético externo é submetido a uma transição $\mathrm{SU}(4)-\mathrm{SU}(2)$ com propriedades muito interessantes e pode operar como um efetivo filtro de spin, estudado a partir de sua condutância, com possíveis aplicações na spintrônica. 
3

\section{Hamiltoniano de Anderson com interação spin-órbita}

\section{1}

\section{Introdução}

Neste capítulo discutiremos os efeitos da incorporação da interação spinorbita (SO) no Hamiltoniano de Anderson e como as propriedades do estado fundamental mudam, em particular a temperatura Kondo $T_{K}$.

Elétrons com momento $\boldsymbol{P}$ na presença de um campo magnético $\boldsymbol{b}$ sofrem a ação da força de Lorentz,

$$
\boldsymbol{F}=-\frac{e}{m} \boldsymbol{P} \times \boldsymbol{b},
$$

e possuem uma energia Zeeman,

$$
B=-\mu_{B} \boldsymbol{\sigma} \cdot \boldsymbol{b},
$$

onde $e$ e $m$ são a carga e a massa dos elétrons, respectivamente, $\mu_{B}$ é o magneton de Bohr e $\boldsymbol{\sigma}$ é um vetor composto pelas matrizes de Pauli $\sigma_{x}, \sigma_{y}, \sigma_{z}$, i.e. $\boldsymbol{\sigma}=\left(\sigma_{x}, \sigma_{y}, \sigma_{z}\right)$. Quando os elétrons movem-se em uma região com campo elétrico $E$ experimentam um campo magnético de origem relativística dado por,

$$
\boldsymbol{b}_{\boldsymbol{r}} \sim \frac{1}{m c^{2}} \boldsymbol{E} \times \boldsymbol{P},
$$

onde $c$ é a velocidade da luz no vácuo. Este campo magnético relativístico introduz uma energia análoga ao desdobramento Zeeman, só que dependente do momento eletrônico, chamado acoplamento spin-órbita (SO),

$$
E_{S O} \sim \frac{\mu_{B}}{m c^{2}}(\boldsymbol{E} \times \boldsymbol{P}) \cdot \boldsymbol{\sigma} .
$$

Em um cristal, o campo elétrico que atua sobre os elétrons pode ser manipulado por meio de um potencial externo $V, \boldsymbol{E}=-\nabla V$, o que permite o controle sobre a magnitude da interação SO. O acoplamento SO surge em sistemas que não possuem simetria de inversão espacial [70], embora conserve a simetria de inversão temporal.

Também podemos introduzir a interação SO diretamente por meio da equação de Dirac,

$$
H_{s o}=-\frac{e \hbar}{4 m^{2} c^{2}}([\nabla V \times \boldsymbol{P}] \cdot \boldsymbol{\sigma}),
$$


onde $\hbar$ é a constante de Planck reduzida $\hbar$. Novamente comparamos a energia associada à interação SO com o desdobramento Zeeman, tomando o produto escalar de $\boldsymbol{\sigma}$ com o vetor originário da correção relativística da interação SO $\nabla V \times P$.

Compostos semicondutores Zinc Blende III-V sem um centro de inversão, tais como GaAs ou InSb, apresentam um acoplamento SO próximo ao ponto $\Gamma$ como observado primeiramente por Dresselhauls [71]. Quando a deformação ocorre na direção (001), estamos no regime de acoplamento SO Dresselhaus linear [70], que possui a forma

$$
H_{D}=\frac{\beta}{\hbar}\left(P_{x} \sigma_{x}-P_{y} \sigma_{y}\right)
$$

onde $\beta$ é uma constante. Já em um poço quântico, a interação SO produzida por um campo elétrico, na direção $z$, que quebra a simetria esférica do potencial, ao qual os elétrons estão sujeitos, na direção $z$ apresenta a forma

$$
H_{R}=-\frac{\alpha}{\hbar} \boldsymbol{z} \cdot(\boldsymbol{\sigma} \times \boldsymbol{P})=-\frac{\alpha}{\hbar}\left(\sigma_{y} P_{x}-\sigma_{x} P_{y}\right),
$$

onde $\alpha$ é o parâmetro Rashba [70, 72-74].

Ambos os acoplamentos Dresselhaus [71] e Rashba [72] acoplam o spin ao momento linear do elétron em sistemas sem centro de simetria de semicondutores zinc-blende (cubo face centrada - fcc) ou wurtzita (hexagonal). Este acoplamento traz grande interesse à interação $\mathrm{SO}$, com desdobramentos em inúmeros fenômenos físicos como o transistor de spin (que discutiremos nesta tese), efeito spin Hall, efeito spin Hall quântico, isolantes topológicos, férmions de Majorana, materiais de Dirac, sistemas de átomos frios, quiralidade em sistemas magnéticos e ondas de spin, torque spin-órbita e bits quânticos [70].

Recentemente surgiu um interesse considerável no estudo do regime Kondo de impurezas magnéticas localizadas interagindo com um substrato sob efeito do acoplamento SO. Esta atividade tem sido estimulada pela importância da interação SO para sistemas com aplicações na spintrônica, como no caso do transistor Datta-Das [75].

A influência da interação SO sobre o efeito Kondo de uma impureza magnética embebida em um sistema bidimensional recebeu muita atenção nos últimos anos [76-83]. Contudo, situações equivalentes onde a impureza é conectada a um condutor unidimensional recebeu uma análise muito mais restritiva [84]. A maioria dos estudos estão limitados a considerar a interação SO atuando somente no volume do sistema, negligenciando os efeitos sobre a própria impureza. Os efeitos da interação SO sobre o regime Kondo de uma impureza magnética ou um ponto quântico (PQ) embebido em substratos de uma ou duas dimensões é um tópico controverso. Enquanto alguns resultados, 
tanto teóricos como experimentais, argumentam sobre a supressão do efeito Kondo [85], outros sustentam a conclusão oposta. Um estudo de uma impureza em um gás bidimensional de elétrons prediz que a temperatura Kondo $T_{K}$ não é afetada pela interação SO [76], enquanto outros autores concluem que os efeitos dependem substancialmente da região no espaço de parâmetros que define o sistema [77]. Alguns trabalhos, usando uma transformação de SchriefferWolff [21] e grupo de renormalização predizem um aumento exponencial da $T_{K}[86,78,83]$. Recentemente, um estudo de uma impureza magnética em um gás bidimensional de elétrons com interação SO Rashba usando o NRG [17] concluiu que a interação SO introduz pequenas variações na $T_{K}$, aumentando ou reduzindo seu valor, que depende dos parâmetros da impureza [77]. Para um fio quântico, onde a interação SO está restrita a produzir somente espalhamentos para frente ou para trás, alguns autores [84] concluíram que a interação SO tem um efeito maior na $T_{K}$ que em sistemas com mais dimensões. Eles foram capazes de concluir, através de uma abordagem do NRG [17], que a $T_{K}$ tem um aumento exponencial.

As diferenças dos sistemas estudados, a variedade dos tratamentos usados e os resultados contraditórios obtidos permitem concluir que um entendimento mais aprofundado da física associada a impurezas magnéticas conectadas à elétrons de condução sob efeito das interações SO se fazem necessárias, requerendo um esforço de síntese e esclarecimento.

Com este propósito, este capítulo é devotado à discutir a influência da interação SO, Rashba [72] e Dresselhaus [71] atuando sobre um mar de Fermi unidimensional conectado ao PQ.

\section{2}

\section{Interação spin-órbita Rashba}

A quebra da simetria na direção $z$ do potencial de confinamento eletrônico origina a interação SO Rashba. Do ponto de vista experimental, a interação SO Rashba é interessante pois pode ser controlada por meio de um potencial externo ao sistema. Este potencial externo aplicado cria um campo elétrico dentro do semicondutor ou metal que destrói parte da sua simetria.

O Hamiltoniano da interação SO Rashba pode ser escrito no modelo de ligações fortes para uma cadeia linear infinita, com as interações restritas aos primeiros vizinhos, como [87]

$$
H_{R}=-\frac{\alpha}{2 a} \sum_{l, \sigma, \sigma^{\prime}} c_{l+1, \sigma^{\prime}}^{\dagger}\left(i \sigma_{y}\right)_{\sigma \sigma^{\prime}} c_{l, \sigma}+\text { h.c. }
$$

onde h.c. significa hermitiano conjugado, $a$ é o parâmetro de rede e $\alpha$ o 
parâmetro Rashba, que mede a intensidade da interação SO Rashba. O operador de criação (aniquilação) no sítio de coordenadas $l$ é $c_{l}^{\dagger}\left(c_{l}\right)$, onde o seu índice, $l$, refere-se a posição do sítio na geometria da rede.

O parâmetro Rashba $\alpha$ é proporcional ao gradiente do potencial externo, $\nabla V$, o que implica que mudando o potencial, altera-se a magnitude da interação SO Rashba.

A figura 3.1 ilustra a propagação dos elétrons ao longo da direção $\boldsymbol{x}$ com um campo elétrico perpendicular na direção $z$ e interação SO Rashba. A direção do spin dos elétrons varia no plano $x z$ devido à interação SO Rashba.
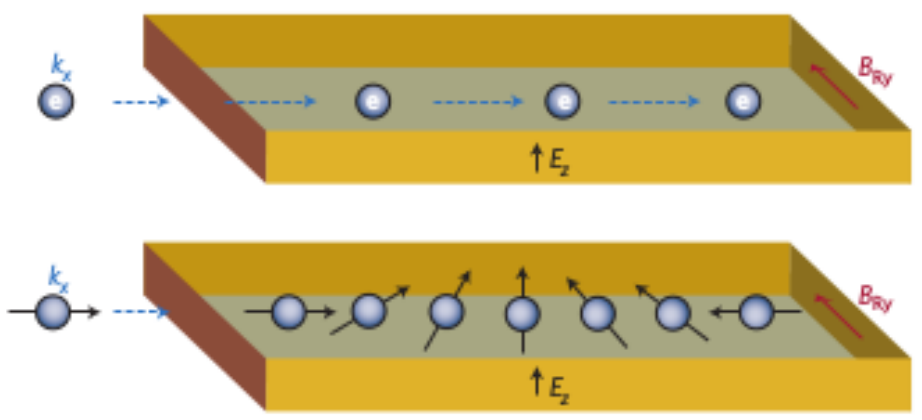

Figura 3.1: Elétrons são injetados ao longo da direção $\boldsymbol{x}$ em uma região onde um campo elétrico atua na direção $z$. Quando o spin dos elétrons injetados não estão alinhados na direção da interação SO Rashba, o spin eletrônico sofre uma precessão como ilustrado no painel inferior.

\section{3}

\section{Interação spin-órbita Dresselhaus}

Em semicondutores sem centros de inversão, e.g. GaAs e InSb, a ausência desta simetria na estrutura cristalina produz um potencial assimétrico, o qual origina a interação SO do tipo Dresselhaus [71]. Diferentemente da interação SO Rashba em que a quebra de simetria dar-se-á por um potencial externo, a interação SO Dresselhaus se origina pela quebra de simetria de rotação completa do próprio potencial cristalino, intrínseco à estrutura da rede sem centro de inversão. O Hamiltoniano do acoplamento SO Dresselhaus na aproximação linear pode ser escrito como

$$
H_{D}=\beta \sum_{l, \sigma, \sigma^{\prime}}\left\{c_{l+1, \sigma^{\prime}}^{\dagger}\left(i \sigma_{x}\right)_{\sigma \sigma^{\prime}} c_{l, \sigma}+\text { h.c. }\right\},
$$

onde $\beta$ é a intensidade da interação SO Dresselhaus. 


\section{4}

\section{Interação spin-órbita no volume}

Consideramos o modelo de ligações fortes para um sistema unidimensional, uma cadeia linear infinita ao longo da direção $x$ com interação SO. A interação SO total neste sistema consiste na soma das interações SO tipo Rashba e Dresselhaus, e é dado de acordo com as equações (3-8) e (3-9) pelo seguinte Hamiltoniano

$$
H_{t o t a l}=-\sum_{l, \sigma, \sigma^{\prime}}\left\{c_{l+1, \sigma}^{\dagger}(i \Gamma)_{\sigma, \sigma^{\prime}} c_{l, \sigma^{\prime}}+\text { h.c. }\right\}
$$

onde $\Gamma$ é a matriz de interação SO definida como,

$$
\Gamma=\left(\begin{array}{cc}
0 & \gamma \\
\gamma^{*} & 0
\end{array}\right),
$$

$\mathrm{e} \gamma=\beta+i \alpha$

O Hamiltoniano que descreve os elétrons na cadeia linear infinita com interação SO é

$$
H_{S O}=-t \sum_{<i, j>, \sigma} c_{i \sigma}^{\dagger} c_{j, \sigma}-\sum_{l, \sigma, \sigma^{\prime}} c_{l+1 \sigma}^{\dagger}(i \Gamma)_{\sigma \sigma^{\prime}} c_{l \sigma}+h . c .,
$$

onde $t$ é o elemento não diagonal da cadeia linear e $<i, j>$ significa o somatório sobre os primeiros vizinhos. A figura 3.2 apresenta um esboço do sistema em estudo.

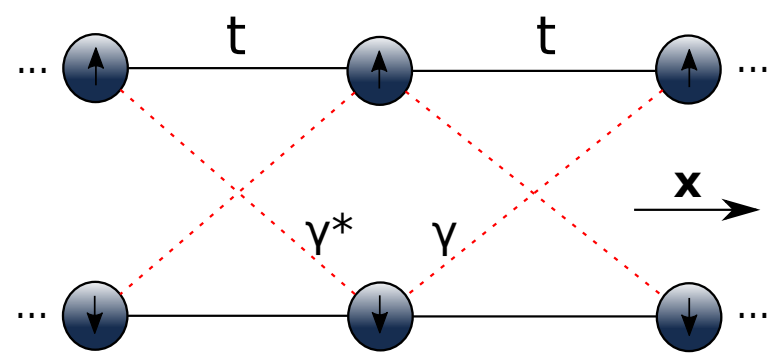

Figura 3.2: Cadeia linear infinita com interação spin-órbita.

Como a cadeia linear possui simetria de translação no eixo $x$, é possível representar a equação (3-12) numa base parcialmente diagonal no espaço recíproco, adotando o número quântico de momento $k=|\boldsymbol{k}|$.

Definindo a transformada de Fourier de $c_{i, \sigma}$ como

$$
c_{i, \sigma}=\frac{1}{\sqrt{N}} \sum_{k, \sigma} e^{i \boldsymbol{k} \cdot \boldsymbol{x}_{i}} c_{k, \sigma},
$$


o primeiro termo da equação (3-12) pode ser reescrito como

$$
\begin{aligned}
& -t \sum_{<i, j>, \sigma} c_{i \sigma}^{\dagger} c_{j, \sigma}=-t \frac{1}{N} \sum_{<i, j>} \sum_{k, \sigma} e^{-i \boldsymbol{k} \cdot\left(\boldsymbol{x}_{\boldsymbol{i}}-\boldsymbol{x}_{\boldsymbol{j}}\right)} c_{k \sigma}^{\dagger} c_{k \sigma}= \\
& -t \frac{1}{N} \sum_{k, \sigma}\left(e^{-i k a}+e^{i k a}\right) c_{k \sigma}^{\dagger} c_{k \sigma}=-\frac{1}{N} \sum_{k, \sigma} 2 t \cos (k a) c_{k \sigma}^{\dagger} c_{k \sigma}
\end{aligned}
$$

onde efetuamos o somatório sobre os primeiros vizinhos. Já o segundo termo resulta em,

$$
-\sum_{k} 2 \operatorname{sen}(k a)\left(\gamma c_{k \uparrow}^{\dagger} c_{k \downarrow}+\gamma^{*} c_{k \downarrow}^{\dagger} c_{k \uparrow}\right) .
$$

Assim, a equação (3-12) pode ser reescrita como,

$$
H_{S O}=-\sum_{k, \sigma} 2 t \cos (k a) c_{k \sigma}^{\dagger} c_{k \sigma}-\sum_{k} 2 \operatorname{sen}(k a)\left(\gamma c_{k \uparrow}^{\dagger} c_{k \downarrow}+\gamma^{*} c_{k \downarrow}^{\dagger} c_{k \uparrow}\right) .
$$

Analisando esta última equação, fica evidente que a presença da interação SO faz com que o sistema deixe de ser diagonal no spin, o que resulta em o spin eletrônico não ser mais um bom número quântico. Diferentemente do primeiro termo da equação (3-16) completamente diagonal nos números quânticos $k$ e $\sigma$, a interação $\mathrm{SO}$ introduz um termo não diagonal em $\sigma$, que deve ser diagonalizado.

A fim de estudar o Hamiltoniano de Anderson na presença de interação $\mathrm{SO}$, necessitamos efetuar uma mudança de base, visto que na base dos spins $\sigma$ a interação SO introduz um termo não diagonal em $\sigma$.

Podemos escrever o Hamiltoniano na base $|k \sigma\rangle$ que descreve a cadeia como,

$$
H_{S O}=-\left(\begin{array}{cc}
2 t \cos (k a) & 2 \gamma \operatorname{sen}(k a) \\
2 \gamma^{*} \operatorname{sen}(k a) & 2 t \cos (k a)
\end{array}\right) .
$$

Os autovalores $\epsilon_{k \nu}$ desta matriz são,

$$
\epsilon_{k \nu}=-2 t \cos (k a)-\nu 2|\gamma||\operatorname{sen}(k a)|
$$

onde definimos o número quântico da helicidade $\nu= \pm$, que surge naturalmente do cálculo dos autovalores. Os autovetores são,

$$
\left|\psi_{k \nu}\right\rangle=\frac{1}{\sqrt{2}}\left(\nu e^{i \phi} \operatorname{sinal}[\operatorname{sen}(k a)]|k \uparrow\rangle+|k \downarrow\rangle\right),
$$

onde sinal $(x)$ é a função sinal de $x$.

A partir da equação (3-19) definimos a transformação

$$
c_{k \nu}^{\dagger}=\frac{1}{\sqrt{2}}\left(\nu e^{i \phi} \operatorname{sinal}[\operatorname{sen}(k a)] c_{k \uparrow}^{\dagger}+c_{k \downarrow}^{\dagger}\right),
$$

que permite escrever o Hamiltoniano em sua forma diagonal na base $\nu$.

A transformação do Hamiltoniano da base dos spins para a base da helicidade é apresentado em detalhes no apêndice C. Nesta nova base o 
Hamiltoniano apresenta a sua forma diagonal, dada por,

$$
H=\sum_{k, \nu} \epsilon_{k \nu} c_{k \nu}^{\dagger} c_{k \nu}
$$

onde a relação de dispersão é,

$$
\epsilon_{k \nu}=-2 t \cos (k a)-2 \nu|\gamma||\operatorname{sen}(k a)| \text {. }
$$

A figura 3.3 mostra o gráfico da relação de dispersão $\epsilon_{k \nu}$ de uma cadeia linear infinita com interação SO e o valor de $|\gamma|=0.5$, expresso em unidades de $t$. Analisando este gráfico observamos que a interação SO modifica consideravelmente a relação de dispersão. Na vizinhança de $k \approx 0$, vemos que os estados com helicidade $\nu=+$ apresentam energia inferior e são em quantidade superior aos estados com helicidade $\nu=-$. Além disso, no ponto $k=0$ a dispersão assume o valor de $-2 t$ para ambos os valores de $\nu$. Também notamos a presença de uma descontinuidade na derivada nos pontos $k=0$ e $k= \pm \pi$ e que a faixa de valores que assume $\epsilon_{k \nu}$ é aumentada pela interação SO. Os estados com energia compreendida entre o mínimo da curva e - $2 t$ são estados com helicidade exclusivamente $\nu=+$. O mesmo acontece no outro extremo entre $2 t$ e o máximo da curva, estados que possuem helicidade exclusivamente $\nu=-$.

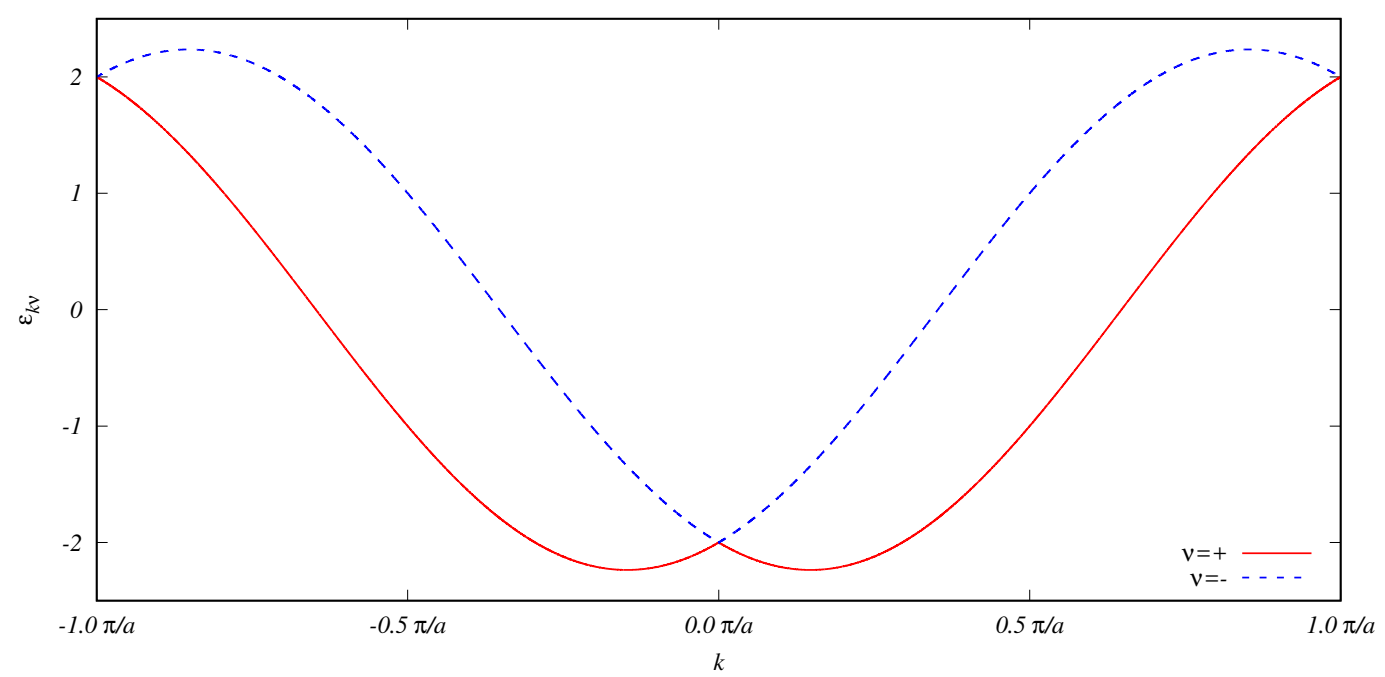

Figura 3.3: Relação de dispersão $\epsilon_{k \nu}$ para cada helicidade $\nu$, equação (3-22). Resultados em unidades de $t$ e com $|\gamma|=0.5$.

A relação de dispersão, equação (3-22), pode ser reescrita, para $k>0$, como

$$
\epsilon_{k \nu}=-2|z| \cos (k a+\nu \Phi)
$$

onde definimos $|z|$ como

$$
|z|=\sqrt{t^{2}+|\gamma|^{2}}
$$


e

$$
\Phi=\operatorname{tg}\left(\frac{|\gamma|}{t}\right) .
$$

Com a relação de dispersão reescrita de acordo com a equação (3-23), podemos obter a densidade de estados deste sistema, que consiste em uma cadeia linear infinita com interação $\mathrm{SO}$, para cada valor de helicidade, i.e. $\rho_{\nu}(\omega)$. Discutiremos o caso com helicidade positiva, mas o mesmo raciocínio se aplica ao caso com helicidade negativa. Na região $-2 t<\epsilon_{k \nu}<2 t$ o resultado é similar ao de uma cadeia linear sem interação $\mathrm{SO}$, já que a forma da relação de dispersão é a mesma (ver figura 3.3) com uma semilargura maior $D=2|z|$. Em $2 t<\epsilon_{k \nu}<2|z|$ não existem estados possíveis para a helicidade $\nu=+$ e portanto a densidade de estados é nula. Já os estados na região $-2|z|<\epsilon_{k \nu}<-2 t$ são contados uma vez a mais que na região $-2 t<\epsilon_{k \nu}<2 t$, resultando em um fator 2 na expressão da densidade de estados. Assim, a expressão para $\rho_{+}(\omega)$ é

$$
\rho_{+}(\omega)=\left\{\begin{array}{ll}
\frac{2}{\pi \sqrt{4|z|^{2}-\omega^{2}}}, & -2|z|<\omega<-2 t \\
\frac{1}{\pi \sqrt{4|z|^{2}-\omega^{2}}}, & -2 t<\omega<2 t \\
0, & 2 t<\omega<2|z|
\end{array} .\right.
$$

O mesmo se aplica para $\nu=-$, resultando em

$$
\rho_{-}(\omega)=\left\{\begin{array}{ll}
0, & -2|z|<\omega<-2 t \\
\frac{1}{\pi \sqrt{4|z|^{2}-\omega^{2}}}, & -2 t<\omega<2 t \\
\frac{2}{\pi \sqrt{4|z|^{2}-\omega^{2}}}, & 2 t<\omega<2|z|
\end{array} .\right.
$$

A densidade de estados $\rho_{\nu}(\omega)$ corresponde à relação de dispersão da equação (3-22), representada no gráfico da figura 3.3.

Como discutido há alguns parágrafos, os estados próximos a $k \approx 0$ são contados uma vez a mais que os no intervalo $-2 t<\omega<2 t$ para $\nu=+$. Isto reflete-se na densidade de estados como uma descontinuidade em $\omega=-2 t$. A menos de um fator $2, \rho_{+}(\omega)$ é a mesma função nos intervalos $-2|z|<\omega<-2 t$ e $-2 t<\omega<2 t$. Por outro lado, a descontinuidade em $\omega=2 t$ originase pela ausência de estados no intervalo de $2 t<\omega<2|z|$. Também é observado pela análise desses dois gráficos, figuras 3.3 e 3.4, que a interação SO amplia a semilargura de banda. A localização das singularidades de VanHove de uma cadeia linear com interação SO, as quais no gráfico da figura 3.3 correspondem aos pontos onde a derivada é nula, são deslocadas de $\pm 2 t$ para $\pm 2|z|$. Conclusões similares também são obtidas para $\rho_{-}(\omega)$.

Obviamente quando tomamos o limite de $\gamma \rightarrow 0$, ou seja, quando a interação SO é eliminada, todos os resultados coincidem com os de uma cadeia 


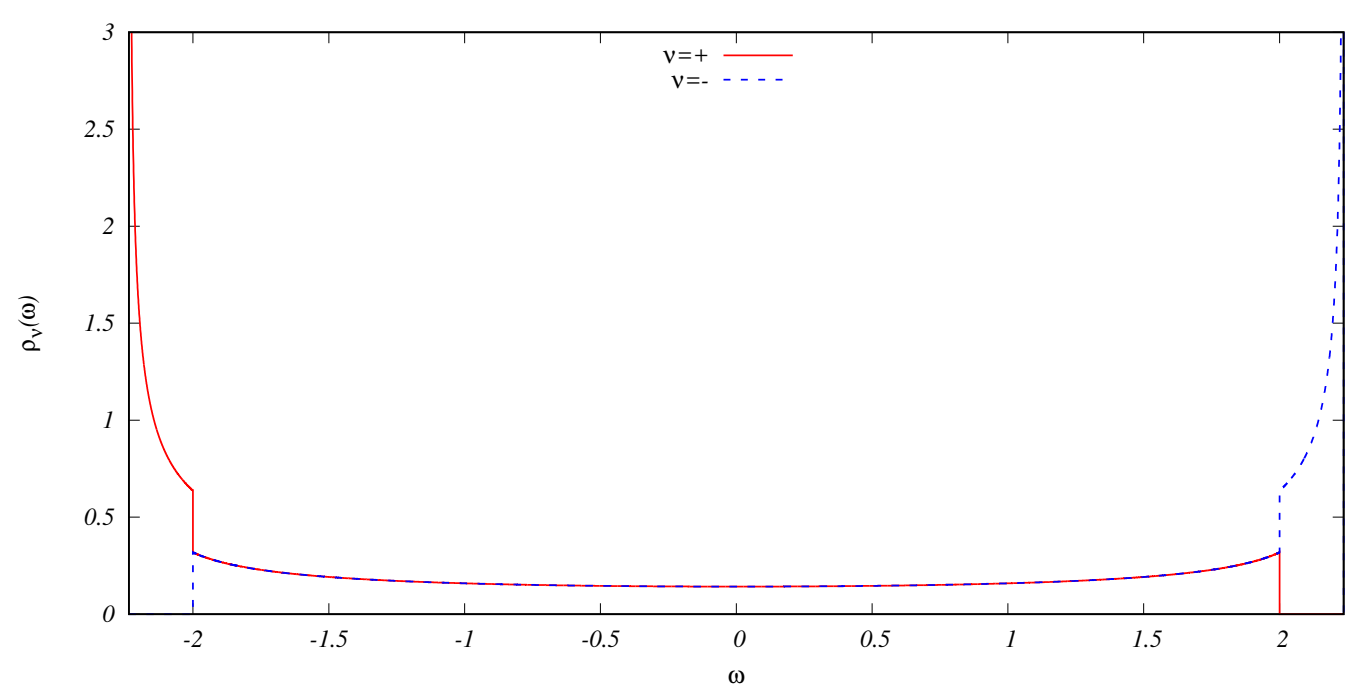

Figura 3.4: Gráfico da densidade de estados $\rho_{\nu}(\omega)$ de uma cadeia linear com interação SO. Mesmos parâmetros da figura 3.3 e em unidades de $t$.

linear infinita usual, os quais são obtidos como um caso particular do sistema tratado aqui.

\section{5}

\section{Influência da interação spin-órbita sobre o efeito Kondo}

Definiremos uma combinação simétrica e antissimétrica com as funções do spin na impureza, $|\uparrow\rangle$ e $|\downarrow\rangle$, do modelo de Anderson,

$$
| \pm\rangle=\frac{1}{\sqrt{2}}\left(|\uparrow\rangle \pm e^{i \phi}|\downarrow\rangle\right)
$$

Estas combinações definem a transformação da impureza,

$$
d_{+}^{\dagger}=\frac{1}{\sqrt{2}}\left(d_{\uparrow}^{\dagger}+e^{i \phi} d_{\downarrow}^{\dagger}\right)
$$

$\mathrm{e}$

$$
d_{-}^{\dagger}=\frac{1}{\sqrt{2}}\left(d_{\uparrow}^{\dagger}-e^{i \phi} d_{\downarrow}^{\dagger}\right),
$$

onde $d_{\sigma}^{\dagger}$ é o operador de criação de um elétron na impureza com spin $\sigma$.

O termo de muitos corpos da repulsão Coulombiana $U$ entre os elétrons na impureza é transformado em,

$$
U n_{\uparrow} n_{\downarrow}=U n_{+} n_{+},
$$

onde $n_{\sigma}=d_{\sigma}^{\dagger} d_{\sigma}$ e $n_{ \pm}=d_{ \pm}^{\dagger} d_{ \pm}$.

As combinações simétrica e antissimétrica se acoplam ao mar de Fermi 
com interação SO na base diagonal como,

$$
\begin{aligned}
\left\langle \pm|H| \psi_{k \nu}\right\rangle & =\frac{1}{2}\left(\nu e^{i \phi} \operatorname{sinal}[\operatorname{sen}(k a)] V+\gamma_{i m p}\right. \\
& \left. \pm \nu \operatorname{sinal}[\operatorname{sen}(k a)] \gamma_{i m p}^{*} \pm e^{-i \phi} V\right),
\end{aligned}
$$

onde definimos $\gamma_{i m p}=\beta_{i m p}+i \alpha_{i m p}$ a interação SO total na conexão entre o mar de Fermi e a impureza

$$
\langle\uparrow|H| k \downarrow\rangle=\gamma_{i m p}
$$

$\mathrm{e}$

$$
\langle\downarrow|H| k \uparrow\rangle=\gamma_{i m p}^{*} .
$$

E $\alpha_{i m p}$ e $\beta_{i m p}$ são os parâmetros Rashba e Dresselhaus, respectivamente, na conexão entre a cadeia linear infinita e a impureza.

Restringiremos a discussão somente a interação SO Rashba no volume e na conexão do mar de Fermi com a impureza, ou seja, $\beta=\beta_{i m p}=0$. Com esta suposição, os únicos termos não nulos no acoplamento da cadeia linear com a impureza são,

$$
\left\langle+|H| \psi_{k<0+}\right\rangle=\left\langle+|H| \psi_{k>0-}\right\rangle=-i\left(V-\alpha_{i m p}\right)
$$

e

$$
\left\langle-|H| \psi_{k<0-}\right\rangle=\left\langle-|H| \psi_{k>0+}\right\rangle=i\left(V+\alpha_{i m p}\right) .
$$

Analisando estas duas equações concluímos que: para uma dada combinação simétrica, $|+\rangle$, ou antissimétrica, $|-\rangle$, ela se acopla para uma helicidade com $k<0$ e com a outra helicidade para $k>0$.

Para ilustrar melhor a curva de dispersão que cada combinação se acopla, refazemos o gráfico da figura 3.3 que contem a relação de dispersão $\epsilon_{k \nu}$ na figura 3.5. Contudo, em vez de graficar $\epsilon_{k \nu}$ para cada helicidade $\nu$, nós iremos graficar a relação de dispersão efetiva ao qual cada combinação se acopla. A combinação simétrica, $|+\rangle$, se acopla a relação de dispersão descrita pela curva número 1 na figura 3.5. Já a combinação antissimétrica, $|-\rangle$, se acopla a curva número 2 .

O módulo quadrado que cada combinação se conecta com o mar de Fermi é,

$$
\left|\left\langle+|H| \psi_{k<0+}\right\rangle\right|^{2}=\left|\left\langle+|H| \psi_{k>0-}\right\rangle\right|^{2}=\left(V-\alpha_{i m p}\right)^{2}
$$

$\mathrm{e}$

$$
\left|\left\langle-|H| \psi_{k<0-}\right\rangle\right|^{2}=\left|\left\langle-|H| \psi_{k>0+}\right\rangle\right|^{2}=\left(V+\alpha_{i m p}\right)^{2} .
$$

Como estas duas últimas equações resultam em conexões de magnitudes diferentes, podemos concluir que a interação SO Rashba que inclua o acoplamento da impureza com o mar de Fermi $\alpha_{i m p}$ quebra a simetria SU(2) necessária para a constituição do efeito Kondo, e que não existe a conexão da impureza 


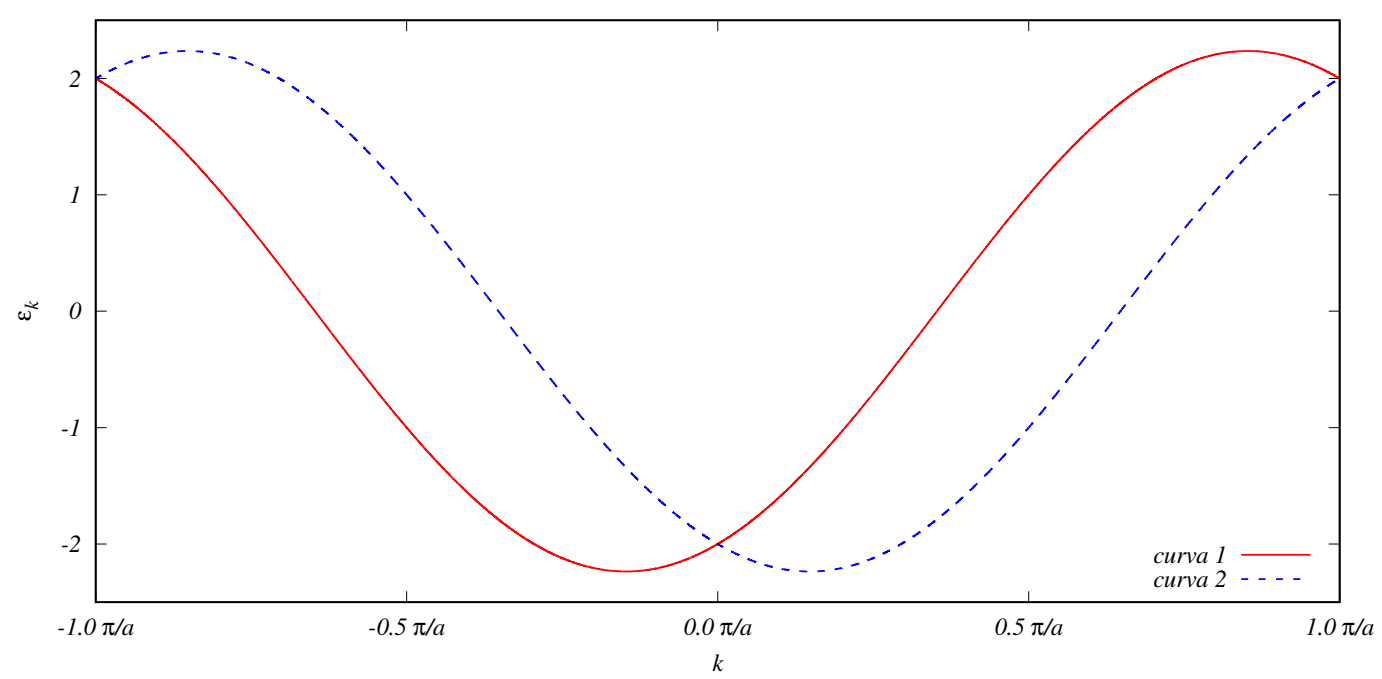

Figura 3.5: Gráfico da relação de dispersão efetiva que cada combinação simétrica ou antissimétrica se acopla. Resultados em unidades de $t$ e com os mesmos parâmetros das figura 3.3.

com o mesmo estado no contínuo. Entretanto, como já analisado no estudo da transição $\mathrm{SU}(4)-\mathrm{SU}(2)$ pode existir para valores pequenos do valor $\alpha_{i m p}$ um estado Kondo emergente que minimiza a energia do sistema.

Por outro lado, se $\alpha_{i m p}=0$, então o efeito Kondo é restabelecido, independentemente do valor da interação SO Rashba no volume, $\alpha$. Neste caso, o sistema não passa de uma cadeia linear com uma semilargura de banda ampliada pela interação SO Rashba acoplada a uma impureza na forma do Hamiltoniano de Anderson. Portanto, a expressão de Haldane [41, 42] para a temperatura Kondo $T_{K}$ é válida.

A interação SO Rashba no volume aumenta a semilargura de banda $2|z|=2 \sqrt{t^{2}+\alpha^{2}}$, o que ocasiona uma diminuição da função de hibridização $\Delta=V^{2} / 2|z|$. Consequentemente o expoente da expressão de Haldane para a $T_{K}$ fica menor,

$$
\begin{aligned}
T_{K} & =\sqrt{\Delta D} \exp \left(\frac{\pi \epsilon_{0}\left(\epsilon_{0}+U\right)}{2 \Delta U}\right) \\
& =V \exp \left(\frac{\pi \sqrt{t^{2}+\alpha^{2}} \epsilon_{0}\left(\epsilon_{0}+U\right)}{V^{2}}\right) .
\end{aligned}
$$

Logo, podemos concluir que a $T_{K}$ diminui exponencialmente pela influência da interação SO Rashba.

Em virtude do exposto, podemos afirmar que a interação SO Rashba reduz a $T_{K}$ em um sistema constituído por uma impureza acoplada a uma cadeia linear infinita com interação SO Rashba. Além disso, se considerarmos 
a conexão entre a impureza e o mar de Fermi com interação SO Rashba, a simetria SU(2) necessária para o efeito Kondo é quebrada e só pode existir como um fenômeno emergente em alguma região para valores pequenos de $\alpha_{i m p}$ comparados com a interação $V$.

O sistema de uma impureza conectada a uma cadeia linear com interação SO Rashba e Dresselhaus no volume já foi teoricamente estudado anteriormente $[84,88]$. Do ponto de vista experimental, a física descrita por estas abordagens teóricas é encontrada em nanofios de InSb [89] e nos estados das bordas unidimensionais de isolantes topológicos [90].

Alguns autores [84] obtiveram um Hamiltoniano Kondo efetivo, o que permitiu efetuar uma análise por grupo de renormalização similar à apresentada para o caso de um gás bidimensional de elétrons com interação SO [78], obtendo a $T_{K}$. Para o caso unidimensional [84], observou-se que a $T_{K}$ sempre aumenta com a magnitude da interação SO, contrastando com nosso resultado apresentado, ao menos no que se refere a somente a interação SO Rashba. Outros autores [88], usando simulações de Hirsch-Fye Monte Carlo quântico e MFSBA concluíram que o efeito Efeito Kondo em um fio quântico é suprimido pelo acoplamento SO Rashba e Dresselhaus, e que a $T_{K}$ é uma função quase linear da energia de SO. Estas conclusões antagônicas revelam que a influência da interação SO sobre o efeito Kondo em sistemas unidimensionais é incerta e um tema controverso.

As conclusões de nosso trabalho a respeito da influência da interação SO Rashba foram obtidas por cálculos puramente analíticos como mostrado neste capítulo. Contudo, nosso resultado só foi possível graças aos avanços propostos pelos autores na referência [84], que propiciaram a diagonalização do Hamiltoniano de maneira apropriada.

Como continuação deste trabalho, destacamos estudar de forma análoga a apresentada somente a influência da interação SO Dresselhaus. Além disso, a generalização dos cálculos para a presença simultânea da interação SO Rashba e Dresselhaus no volume e no acoplamento com a impureza. 


\section{4 \\ Corrente de spin polarizada em um ponto quântico conectado a um mar de Fermi com interação spin-órbita}

\section{1 Introdução}

A spintrônica tem sido um campo de pesquisa de intensa atividade nos últimos anos, baseado na ideia de que o spin eletrônico pode ser utilizado como um grau de liberdade para armazenar e processar informação. Com a crescente miniaturização dos dispositivos eletrônicos, a manipulação do spin emergiu como uma alternativa promissora para resolver as dificuldades que são intrínsecas da eletrônica usual baseada somente na carga eletrônica [91]. Esta abordagem usa fenômenos físicos dependentes do spin que ocorrem nos dispositivos eletrônicos usuais, como a magnetoresistência gigante, para aumentar a velocidade de processamento de dados e reduzir o consumo de energia elétrica com relevância potencial na computação clássica [92-96]. Além disso, um sistema de dois níveis, ingrediente fundamental na computação quântica, pode naturalmente ser construído considerando o grau de liberdade do spin [97]. Mais recentemente, um ramo da spintrônica emergiu como um importante e promissor campo de pesquisa, a spin-orbitrônica, a qual utiliza o acoplamento spin-órbita ( $\mathrm{SO}$ ) para manipular as propriedades de transporte de equilíbrio e não-equilíbrio dependentes do spin [70]. Neste contexto, o transistor de spin semicondutor proposto por Datta-Das [75] resulta ser muito interessante. Ele utiliza as propriedades do acoplamento SO Rashba para induzir, de uma maneira controlada, a precessão do spin eletrônico ao longo do dispositivo. A injeção e detecção de uma corrente de spin polarizada é possível usando contatos ferromagnéticos, enquanto a orientação do spin eletrônico pode ser mudada continuamente conforme a carga eletrônica atravessa a região contendo um gás bidimensional de elétrons em um semicondutor de gap estreito, sobre influência do acoplamento SO.

O acoplamento SO Rashba [72], embora não quebra a simetria de inversão temporal como é o caso do campo magnético, introduz novos níveis de energia, o que requer a introdução de um novo número quântico, a helicidade eletrônica, para diagonalizar o Hamiltoniano [84]. A intensidade do acoplamento SO que 
produz este efeito e a precessão do spin eletrônico pode ser controlada por um campo elétrico perpendicular aplicado ao sistema [72, 98], fornecendo, em princípio, as condições necessárias para construir uma válvula de spin [95].

O termo transistor origina-se da junção das palavras transfer e resistor, é um componente chave na construção de circuitos integrados e é utilizado principalmente como um interruptor ou amplificador. Enquanto que na eletrônica usual o transistor consiste em um dispositivo semicondutor capaz de controlar uma corrente de alta intensidade entre dois terminais, através de uma corrente de intensidade muito mais fraca no terminal da corrente de base do transistor, um spin transistor utiliza o grau de liberdade do spin eletrônico com o propósito de controlar com um potencial externo a polarização de spin da corrente que circula no sistema

Neste capítulo, nosso objetivo é discutir e estudar as propriedades de transporte de uma nanoestrutura composta por um ponto quântico (PQ) acoplado ao mar de Fermi sob o efeito da interação SO e conectado a outros dois contatos. Um dispositivo que mostraremos possuir propriedades similares ao transistor de spin semicondutor Datta-Das. Na nanoestrutura proposta, o potencial de porta aplicado sobre o PQ funciona de forma análoga à corrente de base do transistor semicondutor usual, permitindo o controle sobre a polarização da corrente. O sistema será descrito por uma generalização do modelo de uma impureza de Anderson (MIA) [7, 84, 99]. Sistemas similares já foram estudados por vários autores na perspectiva da análise que o acoplamento SO tem sobre o regime Kondo [84, 76-83]. Contudo, nosso foco está relacionado ao comportamento do sistema acima da temperatura Kondo [7].

O PQ é introduzido de tal modo que permite selecionar com grande acurácia, manipulando o potencial de porta, $\epsilon_{0}$, a energia dos elétrons que o atravessam. O PQ funciona como um filtro de energia que controla o processo de mudança do spin produzido pela interação SO, incorporando o termo Rashba [72], como no dispositivo original de Datta-Das [75]. Nós supomos o mar de Fermi ao qual o PQ está conectado como sendo uma nanofaixa estreita de grafeno de nanômetros de largura, que tem sido provado ser um condutor para o caso de bordas zigzag, com ordenamento ferromagnético nas arestas [100], ou mais convenientemente uma faixa de grafeno depositada sobre uma superfície de níquel $\mathrm{Ni}(111)$ magnetizado. Este último sistema possui uma interação Rashba muito grande devido à grande assimetria da distribuição de carga na interface entre o grafeno e o níquel, e os estados do grafeno tornamse fortemente polarizados magneticamente devido a sua grande hibridização com os estados magnéticos 3d do níquel [101]. A nanofaixa pode ser dopada e a posição do nível de Fermi modificada alterando a carga contida nela pela 
aplicação de um potencial de porta entre o substrato de níquel e a nanofaixa.

Nós estudamos duas configurações diferentes. Um contato ferromagnético é utilizado para injetar corrente de spin polarizada no PQ conectado ao mar de Fermi com acoplamento SO. O contato que recebe a corrente eletrônica pode ser ferromagnético com polarização antiparalela a direção da fonte ferromagnética, ou um contato normal sem magnetização. O processo de inversão do spin é restrito ao PQ, que é caracterizado por um nível de energia local bem definido, $\epsilon_{0}$, que pode ser manipulado por um potencial externo. Apesar de configurações diferentes da que estamos propondo, o problema de um PQ conectado a um substrato com acoplamento SO, acima da temperatura Kondo, foi antes analisado nas referências $[102,103]$. Outros autores estudaram um sistema contendo um mecanismo de inversão de spin introduzido pela aplicação de um campo magnético transversal no PQ [104]. Nosso objetivo aqui é diferente. O sistema que propomos permite aproveitar as propriedades de filtro de energia do PQ que é conseguido manipulando seu potencial de porta, com o objetivo de controlar de uma forma muito eficiente a polarização da corrente que circula pelo dispositivo.

Nós tratamos os aspectos da física de muitos corpos do sistema adotando o Hamiltoniano da impureza de Anderson [40] na aproximação Hubbard III no contexto da analogia da liga. Não obstante a sua simplicidade formal, ela descreve de uma forma apropriada as propriedades de transporte do regime de bloqueamento de Coulomb que esses sistemas possuem [105].

\section{2}

\section{Descrição do sistema}

O sistema que estudamos age como um transistor de spin nanométrico, que consiste em um PQ conectado a um sistema unidimensional sobre efeitos do acoplamento SO e lateralmente a um ou dois contatos ferromagnéticos, com um spin bem definido, através do qual a corrente eletrônica circula. Conforme mostrado na figura 4.1, uma corrente de spin polarizada é introduzida no PQ através do contato da esquerda [106, 107], enquanto a polarização da corrente saindo pelo contato da direita é estudada como função do potencial de porta aplicado ao PQ. Este contato da direita pode ser ferromagnético, com polarização antiparalela ao contato da esquerda, ou não magnético. $O$ dispositivo permite a inversão do spin do elétron circulante devido à interação do PQ com o mar de Fermi da nanofaixa com acoplamento SO. Nós mostramos que a operação do sistema pode ser sintonizada com grande precisão por um potencial de porta aplicado ao PQ.

Este sistema é modelado por uma extensão do MIA, que inclui o 
(a)

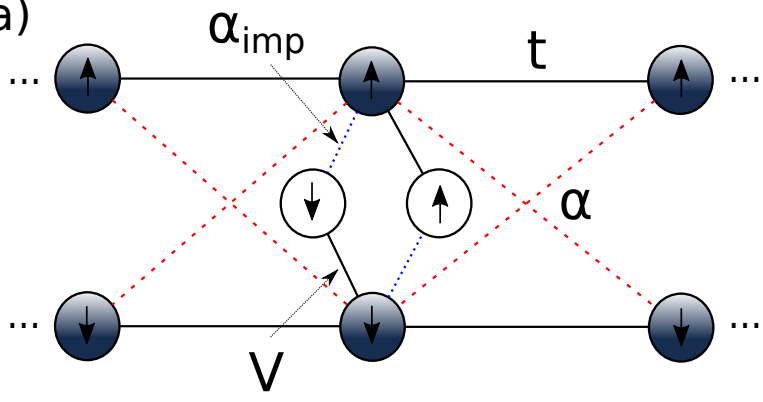

II

(b)

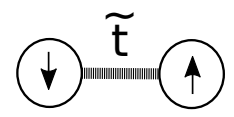

(c)

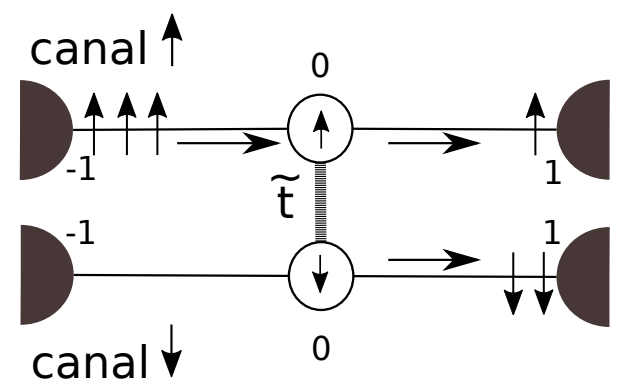

Figura 4.1: (a) Diagrama do PQ (círculos vazios) acoplado a um condutor unidimensional infinito com interação SO no espaço e na representação de spin (círculos azuis preenchidos). Esta estrutura em (b) é representada por dois círculos vazios com uma interação efetiva, $\tilde{t}$, entre eles. Em (c) nós representamos a estrutura em (b) conectada aos contatos -1 e 1 , onde a corrente flui. O spin eletrônico é indicado em cada canal e as setas horizontais representam a corrente eletrônica.

acoplamento Rashba [7, 84, 99], dado por

$$
H=H_{S O}+H_{P Q}+H_{c}+H_{P Q-S O}+H_{P Q-c},
$$

onde

$$
\begin{gathered}
H_{S O}=\sum_{k, \sigma}\left(\epsilon_{k \sigma} n_{k \sigma}+\alpha_{k} c_{k \sigma}^{\dagger} c_{k \bar{\sigma}}\right), \\
H_{P Q}=\sum_{\sigma}\left(\epsilon_{0} n_{\sigma}+\frac{U}{2} n_{\sigma} n_{\bar{\sigma}}\right), \\
H_{c}=\sum_{k, \sigma, i} \epsilon_{k \sigma}^{i} f_{k \sigma, i}^{\dagger} f_{k \sigma, i}, \\
H_{P Q-S O}=\sum_{k, \sigma}\left(V_{k} c_{k \sigma}^{\dagger} d_{\sigma}+V_{k} d_{\sigma}^{\dagger} c_{k \sigma}+\alpha_{i m p}^{\sigma \bar{\sigma}} d_{\sigma}^{\dagger} c_{k \bar{\sigma}}+\alpha_{i m p}^{\bar{\sigma} \sigma} c_{k \bar{\sigma}}^{\dagger} d_{\sigma}\right), \\
H_{P Q-c}=\sum_{k, \sigma, i} t_{k}^{\prime}\left(f_{k \sigma, i}^{\dagger} d_{\sigma}+d_{\sigma}^{\dagger} f_{k \sigma, i}\right) .
\end{gathered}
$$

O primeiro termo do Hamiltoniano, equação (4-2), descreve a nanofaixa com acoplamento SO. O operador ocupação eletrônica é $n_{k \sigma}=c_{k \sigma}^{\dagger} c_{k \sigma}$ e $c_{k \sigma}^{\dagger}\left(c_{k \sigma}\right)$ 
é o operador de criação (aniquilação) de um elétron com momento $k$ e spin $\sigma$. A energia $\epsilon_{k \sigma}=-2 t \cos (k a)+\sigma \delta(\sigma= \pm 1$ corresponde às duas direções do spin) é o elemento diagonal de uma nanofaixa, com elemento de matriz $t$ e parâmetro de rede $a$. Nós incorporamos, através do parâmetro $\delta$, adotando o modelo de Stoner, a magnetização da nanofaixa produzida pelo substrato de níquel, $\mathrm{Ni}(111)$. A quantidade $\alpha_{k}$ tem a forma $\alpha_{k}=-2 \alpha \operatorname{sen}(k a)$, onde $\alpha$ corresponde a intensidade do acoplamento SO Rashba [72]. O segundo termo do Hamiltoniano, equação (4-3), caracteriza o PQ com operador número de ocupação $n_{\sigma}=d_{\sigma}^{\dagger} d_{\sigma}$ e $d_{\sigma}^{\dagger}\left(d_{\sigma}\right)$ é o operador de criação (aniquilação) de um elétron no $\mathrm{PQ}$ com spin $\sigma$. $\mathrm{O}$ outro termo na equação (4-3) corresponde à repulsão Coulombiana intra-PQ caracterizada pelo parâmetro $U$. A equação (4-4) representa os contatos pelos quais os elétrons circulam. O operador $f_{k \sigma, i}^{\dagger}\left(f_{k \sigma, i}\right)$ cria (aniquila) um elétron com spin $\sigma$ e momento $k$ de energia $\epsilon_{k \sigma}^{i}$ nos contatos $i$ à direita ou à esquerda. Nós adotamos o modelo de Stoner para representar o caráter ferromagnético dos contatos supondo que a energia do elétron no contato $i$ dependa do spin. Finalmente, a hibridização entre o PQ e o mar de Fermi com o acoplamento SO e os contatos laterais são dados pelas equações (4-5) e (4-6), respectivamente. O primeiro elemento de matriz incorpora o $V_{k}$ padrão e a contribuição não diagonal Rashba dada por $\alpha_{i m p}^{\sigma \bar{\sigma}}$, enquanto $t_{k}^{\prime}$ define a interação entre o PQ e os contatos. As quantidades $V_{k}=V$ e $t_{k}^{\prime}=t^{\prime}$, por simplicidade, são supostas serem independentes de $k$. O elemento de matriz $\alpha_{i m p}^{\sigma \bar{\sigma}}$ é definido pela matriz $i \sigma_{y} \alpha_{i m p}$, onde $\sigma_{y}$ é a matriz de Pauli e $\alpha_{i m p}$ é a intensidade do acoplamento SO Rashba no PQ, em princípio diferente do acoplamento SO Rashba no volume, $\alpha$ [72].

\section{3}

Teoria

As propriedades do sistema são calculadas usando o formalismo das funções de Green [38]. Em particular, os efeitos de muitos corpos introduzidos pela repulsão Coulombiana intra-ponto são tratados no escopo da aproximação conhecida como analogia da liga Hubbard III [40, 105]. Apesar de sua simplicidade, esta é uma abordagem apropriada quando o sistema encontra-se acima da temperatura Kondo [7, 8]. Ela descreve corretamente as propriedades condutoras no regime de bloqueamento de Coulomb [40]. Infelizmente, este não é o caso de alguns tratamentos que calculam as funções de Green usando o método de equação de movimento, com um esquema de desacoplamento aplicado a primeira série de equações de movimento, a chamada aproximação Hubbard I [108]. Esta é uma questão sutil, já que esta aproximação não é capaz de descrever a condutância do sistema no regime de bloqueamento de Coulomb [109]. 
$\mathrm{Na}$ situação de ressonância $\epsilon_{0}=\epsilon_{F}$ ou $\epsilon_{0}+U=\epsilon_{F}$, onde $\epsilon_{F}$ é a energia de Fermi do sistema, a condutância resulta ser, nesta aproximação, independente da ocupação eletrônica no PQ, eliminando todo o efeito do bloqueamento de Coulomb. É evidente que a entrada de um elétron com spin $\sigma$ no PQ e seu deslocamento pelo sistema depende da ocupação eletrônica de spin oposto no PQ, devido à repulsão Coulombiana.

Os números de ocupação no PQ são calculados, no equilíbrio termodinâmico ou muito próximo a ele, usando o formalismo das funções de Green,

$$
\left\langle n_{\sigma}\right\rangle=\frac{1}{\pi} \int_{-\infty}^{\epsilon_{F}} \Im\left\{G_{00}^{\sigma \sigma}(\omega)\right\} d \omega,
$$

onde $\epsilon_{F}$ é a energia de Fermi e $\Im$ significa parte imaginária. A função de Green no PQ foi obtida aplicando a abordagem da equação de movimento. A condutância eletrônica, devido ao regime de resposta linear do sistema a um potencial externo, é estudada usando o formalismo de Keldysh [69] aplicado ao caso de um sistema com uma região de interação eletrônica [68]. O limite atômico das funções de Green do PQ sobre efeito da interação Coulombiana repulsiva intra-ponto pode ser escrito como;

$$
g_{0}^{\sigma}=\left(1-n_{\bar{\sigma}}\right) g_{0,1}+n_{\bar{\sigma}} g_{0,2},
$$

onde,

$$
\begin{gathered}
g_{0,1}=\frac{1}{\omega-\epsilon_{0}-i \eta} . \\
g_{0,2}=\frac{1}{\omega-\epsilon_{0}-U-i \eta} .
\end{gathered}
$$

Dentro da aproximação da analogia da liga Hubbard III, a equação (4-8) pode ser pensada como expressando que a probabilidade $P_{1}$ de ter um PQ sem ocupação eletrônica, caracterizado por uma energia local $\epsilon_{0}$ é $P_{1}=1-n_{\bar{\sigma}}$, enquanto que o PQ já ocupado por um elétron, com energia local $\epsilon_{0}+U$, a probabilidade seria $P_{2}=n_{\bar{\sigma}}$. Neste caso a interação do PQ com o resto do sistema seria incluída renormalizando as energias locais do respectivo $g_{0, j} \mathrm{e}$ tomando, com as devidas probabilidades acima, o valor médio das funções de Green vestidas [105].

Nesta perspectiva, nós resolvemos o problema em dois estágios. No primeiro, nós omitimos a conexão do sistema com os contatos laterais (onde a corrente polarizada de spin é introduzida), assumindo $t^{\prime}=0$. Assim, a função de Green satisfaz,

$$
\begin{gathered}
G_{00, j}^{\sigma \sigma}=g_{0, j}+g_{0, j} V \sum_{k} G_{k 0, j}^{\sigma \sigma}+\alpha_{i m p} g_{0, j} \sum_{k} G_{k 0, j}^{\bar{\sigma} \sigma}, \\
G_{k 0, j}^{\sigma \sigma}=g_{k}^{\sigma} V G_{00, j}^{\sigma \sigma}+g_{k}^{\sigma} \alpha_{i m p}^{\sigma \bar{\sigma}} G_{00, j}^{\bar{\sigma} \sigma}+g_{k}^{\sigma} \alpha_{k} G_{k 0, j}^{\bar{\sigma} \sigma},
\end{gathered}
$$




$$
G_{k 0, j}^{\bar{\sigma} \sigma}=g_{k}^{\bar{\sigma}} V G_{00, j}^{\bar{\sigma} \sigma}+g_{k}^{\bar{\sigma}} \alpha_{i m p}^{\bar{\sigma} \sigma} G_{00, j}^{\sigma \sigma}+g_{k}^{\bar{\sigma}} \alpha_{k} G_{k 0, j}^{\sigma \sigma}
$$

$\mathrm{e}$

$$
g_{k}^{\sigma}=\frac{1}{\omega-\epsilon_{k \sigma}-i \eta} .
$$

A solução do sistema de equações acima permite reescrever a função de Green diagonal vestida, equação 4-11, em uma forma compacta como,

$$
G_{00, j}^{\sigma \sigma}=\tilde{g}_{0, j}^{\sigma}+\tilde{g}_{0, j}^{\sigma} \tilde{t} G_{00, j}^{\bar{\sigma} \sigma}
$$

onde a função de Green renormalizada diagonal no spin $\tilde{g}_{0, j}$ é dada por,

$$
\tilde{g}_{0, j}^{\sigma}=\frac{g_{0, j}}{1-g_{0, j}^{\sigma}\left(V^{2} \sum_{k} \tilde{g}_{k}^{\sigma}+\alpha_{i m p}^{2} \sum_{k} \tilde{g}_{k}^{\bar{\sigma}}\right)}
$$

$\mathrm{e}$

$$
\tilde{g}_{k}^{\sigma}=\frac{g_{k}^{\sigma}}{1-g_{k}^{\sigma} \alpha_{k}^{2} g_{k}^{\bar{\sigma}}}
$$

A interação renormalizada entre os diferentes estados de spin no PQ, chamada de $\tilde{t}$, é dada por,

$$
\tilde{t}=V \alpha_{i m p} \sum_{k}\left(\tilde{g}_{k}^{\bar{\sigma}}-\tilde{g}_{k}^{\sigma}\right)+\left(V^{2}-\alpha_{i m p}^{2}\right) \sum_{k} \frac{\alpha_{k} g_{k}^{\bar{\sigma}} g_{k}^{\sigma}}{1-g_{k}^{\bar{\sigma}} \alpha_{k}^{2} g_{k}^{\sigma}} .
$$

É necessário incluir as interações com os contatos laterais, previamente desprezadas. Nós reescrevemos a equação (4-15) que contém a informação dos termos do acoplamento SO nas renormalizações, i.e. $\tilde{g}_{0, j}^{\sigma}$ e $\tilde{t}$, e obtemos,

$$
G_{00, j}^{\sigma \sigma}=\tilde{g}_{0, j}^{\sigma}+\tilde{g}_{0, j}^{\sigma} \tilde{t} G_{00, j}^{\bar{\sigma} \sigma}+\tilde{g}_{0, j}^{\sigma} t^{\prime} G_{-10, j}^{\sigma \sigma}+\tilde{g}_{0, j}^{\sigma} t^{\prime} G_{10, j}^{\sigma \sigma},
$$

onde,

$$
\begin{aligned}
G_{10, j}^{\sigma \sigma} & =\tilde{g}_{1}^{\sigma} t^{\prime} G_{00, j}^{\sigma \sigma}, \\
G_{-10, j}^{\sigma \sigma} & =\tilde{g}_{-1}^{\sigma} t^{\prime} G_{00, j}^{\sigma \sigma}, \\
G_{10, j}^{\bar{\sigma} \sigma} & =\tilde{g}_{1}^{\bar{\sigma}} t^{\prime} G_{00, j}^{\bar{\sigma} \sigma}
\end{aligned}
$$

$\mathrm{e}$

$$
G_{-10, j}^{\bar{\sigma} \sigma}=\tilde{g}_{-1}^{\bar{\sigma}} t^{\prime} G_{00, j}^{\bar{\sigma} \sigma}
$$

A função de Green renormalizada, $\tilde{g}_{i}$, que aparece nas equações acima, é vestida pelo contato $i$, que por simplicidade é tomado como representado por uma densidade de estados ferromagnética semi-circular, a qual pode ser associada a uma cadeia linear semi-infinita. Resolvendo este sistema de equações, (4-19) a (4-23), nós obtemos a função de Green no PQ e diagonal no spin dada por,

$$
G_{00, j}^{\sigma \sigma}=\frac{\tilde{g}_{0, j}^{\sigma}}{1-\frac{\tilde{g}_{0, j}^{\sigma} \tilde{t}^{2} \tilde{g}_{0, j}^{\bar{\sigma}}}{1-\tilde{g}_{0, j}^{\bar{\sigma}} t^{\prime 2}\left(\tilde{g}_{-1, j}^{\bar{\sigma}}+\tilde{g}_{1, j}^{\bar{\sigma}}\right)}-\tilde{g}_{0, j}^{\sigma} t^{\prime 2} \tilde{g}_{-1, j}^{\sigma}-\tilde{g}_{0, j}^{\sigma} t^{\prime 2} \tilde{g}_{1, j}^{\sigma}}
$$

e a não diagonal em spin, 


$$
G_{00, j}^{\bar{\sigma} \sigma}=\frac{\tilde{g}_{0, j}^{\bar{\sigma}}}{1-\tilde{g}_{0, j}^{\bar{\sigma}} t^{\prime 2}\left(\tilde{g}_{-1, j}^{\bar{\sigma}}+\tilde{g}_{1, j}^{\bar{\sigma}}\right)} \tilde{t}^{*} G_{00, j}^{\sigma \sigma} .
$$

Finalmente, na aproximação Hubbard III, as funções de Green spin diagonal e não diagonal no PQ, necessárias para calcular a corrente, podem ser escritas como,

$$
\begin{aligned}
G_{00}^{\sigma \sigma} & =\sum_{j} P_{j} G_{00, j}^{\sigma \sigma} \\
& =\sum_{j} P_{j} \frac{\tilde{g}_{0, j}^{\sigma}}{1-\frac{\tilde{g}_{0, j}^{\sigma} \mid \tilde{t}^{2} \tilde{g}_{0, j}^{\bar{\sigma}}}{1-\tilde{g}_{0, j}^{\bar{\sigma}} t^{2}\left(\tilde{g}_{-1, j}^{\bar{\sigma}}+\tilde{g}_{1, j}^{\bar{\sigma}}\right)}-\tilde{g}_{0, j}^{\sigma} t^{\prime 2} \tilde{g}_{-1, j}^{\sigma}-\tilde{g}_{0, j}^{\sigma} t^{\prime 2} \tilde{g}_{1, j}^{\sigma}}
\end{aligned}
$$

$\mathrm{e}$

$$
G_{00}^{\bar{\sigma} \sigma}=\sum_{j} P_{j} G_{00, j}^{\bar{\sigma} \sigma}=\sum_{j} P_{j} \frac{\tilde{g}_{0, j}^{\bar{\sigma}}}{1-\tilde{g}_{0, j}^{\bar{\sigma}} t^{\prime 2}\left(\tilde{g}_{-1, j}^{\bar{\sigma}}+\tilde{g}_{1, j}^{\bar{\sigma}}\right)} \tilde{t}^{*} G_{00, j}^{\sigma \sigma} .
$$

O dispositivo é considerado estar sob o efeito de um potencial externo infinitesimal. Neste caso, os números de ocupação no PQ, $\left\langle n_{\sigma}\right\rangle$, são calculados resolvendo autoconsistentemente a equações (4-7) e (4-26). A corrente eletrônica para cada spin $\sigma, J_{\sigma}$, que flui do contato da esquerda, $i=-1$ na figura 4.1 para o da direita, $i=1$, é calculada usando o formalismo de Keldysh [69]. Por uma questão de clareza, a corrente é injetada através do contato ferromagnético na esquerda totalmente polarizada no spin. No PQ, os elétron sofrem o processo de inversão de spin devido ao acoplamento SO Rashba e saem do ponto através do contato da direita parcialmente polarizados. A corrente é obtida como,

$$
J^{\sigma}=\frac{e^{2}}{\hbar} t^{\prime 2} \int_{-\infty}^{\infty}\left(\bar{G}_{10}^{-+}-\bar{G}_{01}^{\sigma \sigma}\right) d \omega
$$

onde $e$ é a carga eletrônica, $\hbar$ a constante de Planck reduzida e $\bar{G}_{10}^{+}$a função de Green de não equilíbrio de Keldysh entre os sítios $i=0$ e $i=1[69,110,111]$. A função de Green de não equilíbrio de Keldysh satisfaz,

$$
\stackrel{-+}{G}=\left(1+G^{(r)} t^{\prime}\right) \stackrel{-+}{g}\left(1+t^{\prime} G^{(a)}\right)
$$

onde o $G$ 's são as matrizes das funções de Green e os sobrescritos $r$ e $a$ significam retardadas e avançadas, respectivamente. 1 é a matriz identidade, $\boldsymbol{t}^{\prime}$ é a matriz definida como $\boldsymbol{t}_{i j}^{\sigma \sigma^{\prime}}=t^{\prime}\left(\delta_{i, 0} \delta_{j, 1}+\delta_{i 0} \delta_{j,-1}\right) \delta_{\sigma, \sigma^{\prime}}$ e $\stackrel{\boldsymbol{g}}{\boldsymbol{g}}^{+}$como $\bar{g}_{i}^{-+}=$ $2 \pi \rho_{i}^{\sigma} f_{i}^{\sigma}\left(\delta_{i, 1}+\delta_{i,-1}\right)$, onde $\rho_{i}^{\sigma}$ e $f_{i}^{\sigma}$ são a densidade de estados eletrônicos e a distribuição de Fermi no sítio $i$ dos contatos, respectivamente. Após alguma álgebra, a expressão final para a condutância dependente do spin, $C^{\sigma}$, no 
regime de resposta linear, pode ser escrita como,

$$
\begin{array}{r}
C^{\sigma}=\frac{e^{2}}{\hbar} 4 \pi^{2} t^{\prime 4}\left(\rho_{-1}^{\sigma}\left(\epsilon_{F}\right) \rho_{1}^{\sigma}\left(\epsilon_{F}\right)\left|G_{00}^{\sigma \sigma}\left(\epsilon_{F}\right)\right|^{2}+\right. \\
\left.\rho_{-1}^{\bar{\sigma}}\left(\epsilon_{F}\right) \rho_{1}^{\sigma}\left(\epsilon_{F}\right)\left|G_{00}^{\bar{\sigma} \sigma}\left(\epsilon_{F}\right)\right|^{2}\right) .
\end{array}
$$

É importante enfatizar, a respeito das expressões analíticas obtidas nesta seção, que o segundo termo da contribuição a autoenergia de inversão de spin, $\tilde{t}$, definida na equação (4-18), é zero por causa da quantidade $\alpha_{k}=-2 \alpha \operatorname{sen}(k a)$ ser uma função ímpar em $k$ de modo que o somatório sobre o momento é nulo. A contribuição a $\tilde{t}$ origina-se do primeiro termo da equação (4-18), proporcional à hibridização $V$, que conserva o spin, e a $\alpha_{i m p}$, intensidade do acoplamento SO Rashba entre o PQ e a nanofaixa de grafeno. Ademais, está claro que este termo contribui porque $\tilde{g}_{k}^{\bar{\sigma}} \neq \tilde{g}_{k}^{\sigma}$. Este é o caso devido à hibridização das bandas d magnetizadas do substrato de níquel, com a banda $\pi$ do grafeno $[101,112]$. Apesar de não essencial para a física estudada, nós assumimos, por simplicidade, $\tilde{g}_{k}^{\bar{\sigma}}\left(\epsilon_{F}\right)=0$, que implica supor que os contatos possuem ao nível de Fermi somente uma banda com elétrons com spin para cima. Outro ponto importante, como discutido em detalhe a seguir, nós adotamos o nível de Fermi muito próximo à singularidade de Van-Hove do mar de Fermi composto pela nanofaixa de grafeno pois isto aumenta substancialmente os efeitos do processo de inversão do spin, maximizando $\tilde{t}$. Isto pode ser conseguido aplicando uma voltagem entre a nanofaixa e o substrato, que permite a transferência de carga entre eles, alterando a posição do nível de Fermi.

\section{4}

Resultados e discussões

Com estas considerações, os resultados são apresentados em unidades de $t$, sendo $4 t$ a largura da banda dos contatos ferromagnéticos. Os valores dos elementos de matriz entre o PQ e os contatos magnéticos e o mar de Fermi com acoplamento SO são tomados como $V=t^{\prime}=0.3$. Além disso, por simplicidade, nós assumimos $\alpha_{i m p}=\alpha$.

A faixa nanométrica de grafeno é representada por uma densidade de estados unidimensional [113, 114], que possui singularidades de Van-Hove. O sistema pode ser sintonizado para que o nível de Fermi se encontre muito próximo a singularidade por meio de uma voltagem aplicada entre o substrato de $\mathrm{Ni}(111)$ e a nanofaixa, permitindo a transferência de carga. Isto é um ingrediente essencial a respeito da física que nós estamos estudando. Na representação de Stoner da magnetização do grafeno, nós assumimos $\delta=0.05$ (valor expresso em unidades de $t$ ), lembrando que a quantidade equivalente 
para o caso do níquel é uma ordem de grandeza maior [115]. De qualquer maneira, o valor preciso de $\delta$ não é essencial, como discutiremos a seguir. É importante discutir as propriedades da região perto da singularidade de VanHove, que para a densidade de estados da nanofaixa corresponde ao momento linear na vizinhança de $k \approx 0$ para um sistema que está sobre o efeito de interação SO e de uma magnetização espontânea produzida pelo substrato de níquel.

Vamos estudar este sistema nas proximidades da singularidade de VanHove, adotando uma abordagem de ligações fortes de uma cadeia linear infinita. Usando como base para o Hamiltoniano os estados de spin para cima e spin para baixo, a fim de escrevê-lo em forma matricial, obtemos,

$$
H=\left(\begin{array}{cc}
\epsilon_{k \sigma}-\delta & \alpha_{k} \\
\alpha_{k} & \epsilon_{k \sigma}+\delta
\end{array}\right),
$$

onde $\epsilon_{k \sigma}=-2 t \cos (k a), \alpha_{k}=-2 \alpha \operatorname{sen}(k a)$ e inserimos o termo $\delta$ nos elementos diagonais devido à magnetização oriunda do substrato de níquel. A relação de dispersão é dada pelos autovalores desta matriz:

$$
\epsilon_{ \pm}=-2 t \cos (k a) \pm \sqrt{\delta^{2}+4 \alpha^{2} \operatorname{sen}^{2}(k a)}
$$

cuja dependência com $k=|\boldsymbol{k}|$ é representada na figura 4.2 (a). Para $\alpha=0$ e $\delta=0$ a relação de dispersão $\epsilon_{ \pm}$se reduz a relação de dispersão tradicional de ligações fortes de um sistema unidimensional. Contudo, $\operatorname{com} \delta=0$ e $\alpha=0.4$, observamos que o acoplamento SO Rashba introduz uma descontinuidade na derivada em ambas as relações de dispersão, e para $\epsilon_{-}$dois mínimos existem nas redondezas de $k \approx 0$. A figura 4.2 (b) mostra uma ampliação da região $k \approx 0$ para $\alpha=0.4$ e diferentes valores de $\delta$, onde notamos que a descontinuidade da derivada deixa de existir devido a $\delta$. Conforme seu valor é aumentado, $\epsilon_{+}$ é deslocado para cima e $\epsilon_{-}$para baixo. As singularidades de Van-Hove estão localizadas nas energias em que a derivada da relação de dispersão é zero. Como apresentado na figura, isto corresponde ao ponto $k=0$ para dois valores diferentes da energia, associados aos dois valores de separação da magnetização $2 t \pm \delta$ e um terceiro mínimo, resultante da interação SO [87] para um valor menor de energia.

Na figura 4.3 nós mostramos a parte real e imaginária de $\tilde{t}(\omega)$, linhas sólida (vermelha) e tracejada (azul), respectivamente. Como pode ser concluído da figura, a autoenergia que permite a inversão do spin, $\tilde{t}(\omega)$, torna-se significativa no valor das singularidades de Van-Hove da nanofaixa de grafeno como acima descritos. Como esta é a função que origina o mecanismo de inversão do spin responsável pela operação do transistor de spin, é crucial para a energia 

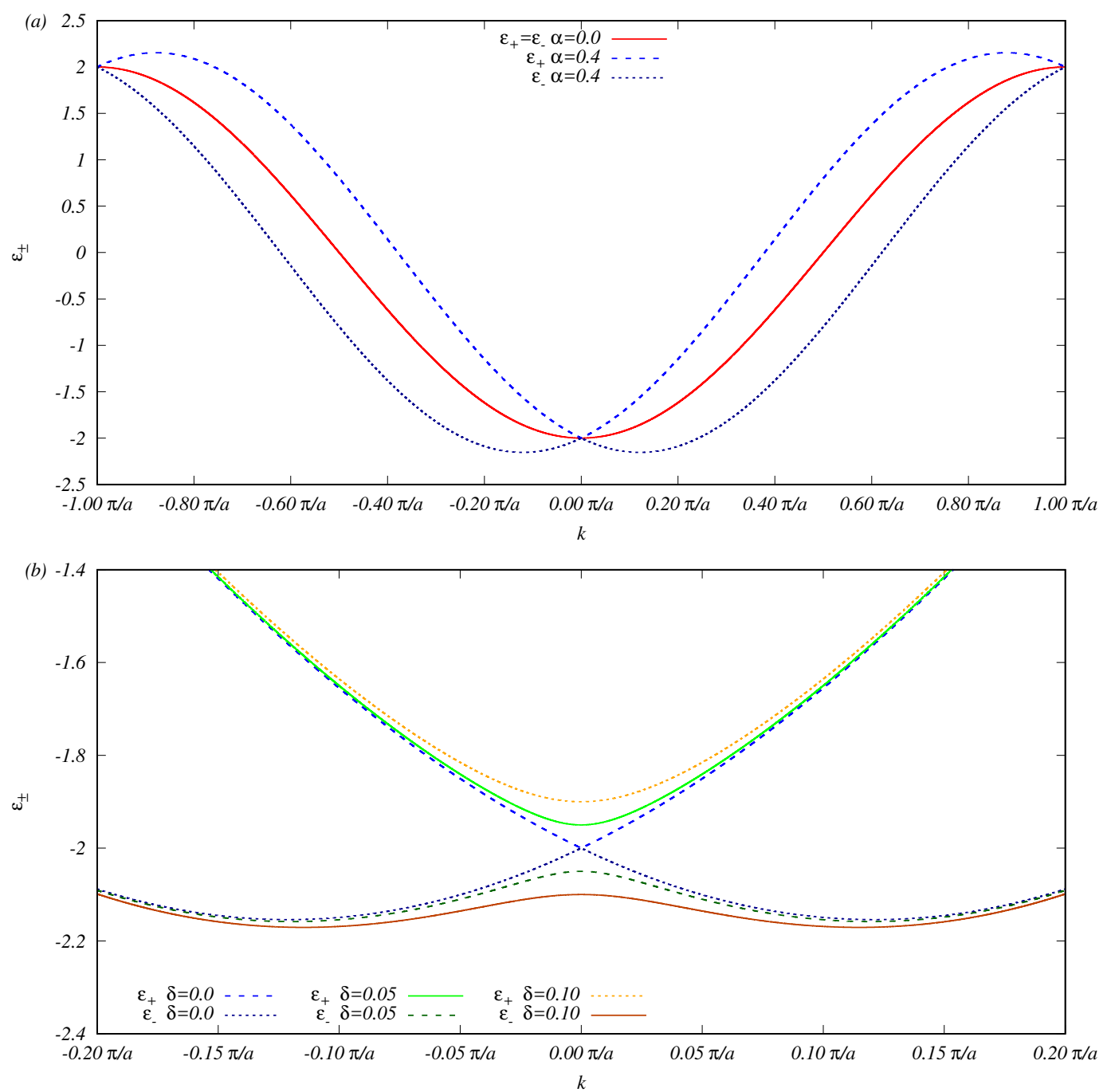

Figura 4.2: O painel (a) mostra a relação de dispersão para $\alpha=0$ e $\alpha=0.4$, ambos com $\delta=0$. No painel (b) a vizinhança de $k=0$ para um valor fixo de $\alpha=0.4$ e vários valores de $\delta$. As valores estão expressos em unidades de $t$.

de Fermi estar localizada nessa região para maximizar os efeitos de inversão do spin. Um potencial externo aplicado entre o substrato de níquel e o grafeno permite a transferência de carga de um subsistema ao outro, que resulta ser uma forma muito acurada de sintonizar a posição do nível de Fermi.

Com o propósito de estudar a operação do transistor de spin e tomando $\delta=0.05$, nós assumimos $\epsilon_{F}=-1.95$, que corresponde a maior energia entre as singularidades de Van-Hove. Investigamos a operação do transistor quando somente os elétrons com spin para cima são injetados no sistema através de um contato ferromagnético e com dois tipos de contatos diferentes. Primeiramente assumimos que a corrente sai do PQ por um contato metálico não magnético, cuja polarização da corrente pode ser manipulada pelo potencial de porta no PQ. Esta configuração é discutida na subseção 4.4.1. Posteriormente, supomos 


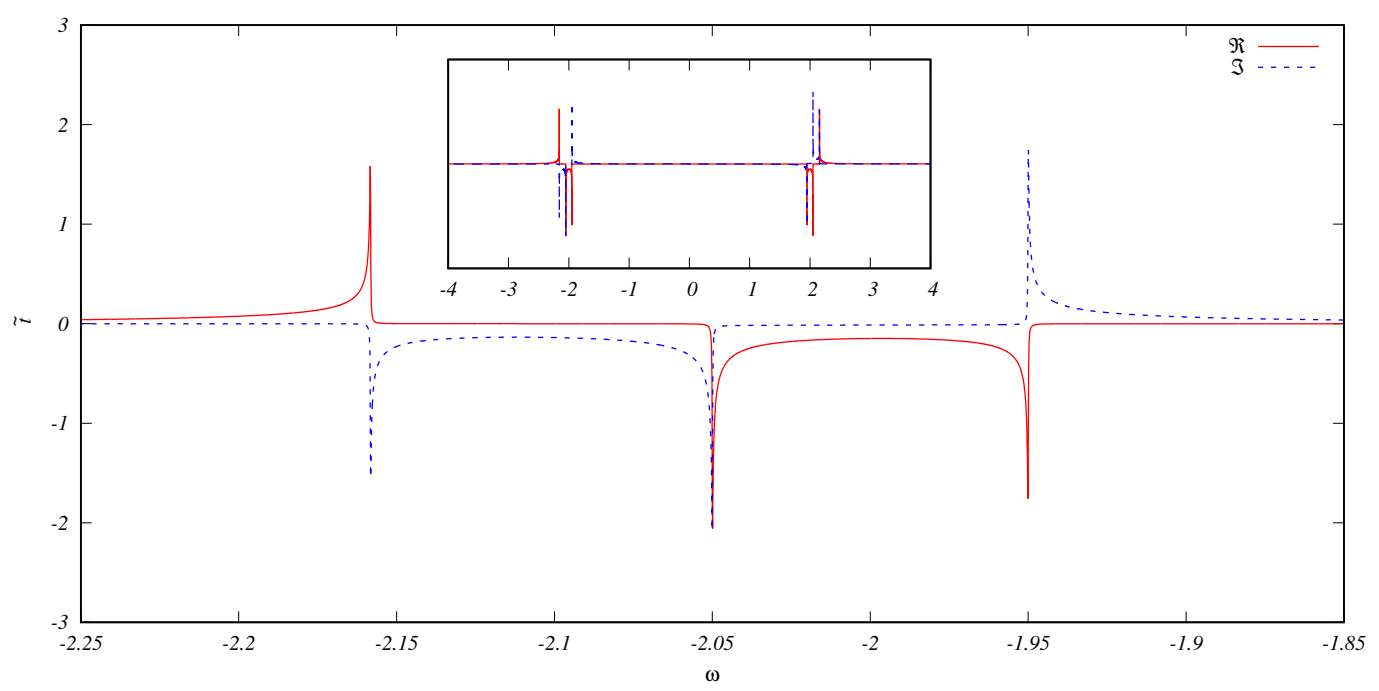

Figura 4.3: Comportamento de $\tilde{t}(\omega)$ para $V=0.3, \alpha=0.4$ e $\delta=0.05$. A parte real está graficada como linha sólida (em vermelho) e a imaginária com a tracejada (em azul). No gráfico menor são apresentados os mesmos resultados, mas para uma escala de $\omega$ maior.

os dois contatos idênticos e feitos de um material ferromagnético, mas com direções de magnetização opostas. Neste caso, a injeção de elétron com spin para cima produz uma condutância emergente completamente polarizada com spin para baixo, como discutido na subseção 4.4.2. Em ambos os casos a performance do dispositivo depende criticamente do potencial de porta do $\mathrm{PQ}$.

\subsection{1}

\section{Contatos ferromagnéticos e não magnéticos}

Nesta configuração, o contato ferromagnético injeta elétrons polarizados com spin para cima através do contato da esquerda, $i=-1$ na figura 4.1, com energia no nível de Fermi, $\epsilon_{F}$, e o dispositivo está conectado a um contato não magnético na direita, $i=1$, através do qual pode circular elétrons com qualquer polarização de spin. Em nosso cálculo, os estados do contato $i=-1$ com spin para baixo são descritos por uma banda completamente vazia ou completamente preenchida fora do nível de Fermi, que se encontra na banda dos elétrons com spin para cima. Neste caso, somente elétrons com spin para cima podem ser injetados no sistema. A banda metálica d do níquel, por exemplo, possui somente um tipo de spin ao nível de Fermi. Como esta é a única propriedade relevante dos contatos, assumimos uma banda semi-circular preenchida até a metade, por simplicidade com spin para cima.

Inicialmente mostraremos os resultados sem repulsão Coulombiana, i.e. 
$U=0$, onde o tratamento se reduz à solução exata de função de Green de um corpo. A figura 4.4 (a) mostra a condutância para cada spin, $C^{\sigma}$, como uma função do potencial de porta $\epsilon_{0}$. As linhas sólida (vermelho) e a pontilhada (azul) correspondem a $C^{\uparrow}$, enquanto que $C^{\downarrow}$ é representada pelas linhas tracejadas (vermelho escuro) e ponto-tracejadas (azul escuro). Os resultados apresentados nesta figura correspondem a dois valores do acoplamento SO Rashba, $\alpha=0.1$ para a curva sólida e tracejadas (em vermelho), enquanto as curvas pontilhada e ponto-tracejada (em azul) para $\alpha=0.4$. Analisando a figura 4.4 (a), é possível observar dois picos na condutância, um em $\epsilon_{0}$ próximo ao $\epsilon_{F}$ e outro em $\epsilon_{0} \approx-0.5$. Enquanto o pico na condutância em $\epsilon_{0}$ próximo ao $\epsilon_{F}$ é esperado já que o PQ está em ressonância com este valor de potencial de porta, o outro pico em $\epsilon_{0} \approx-0.5$ é novo, e sua origem é um resultado das singularidades da autoenergia $\tilde{t}(\omega)$ que inverte o spin devido ao acoplamento SO Rashba. Investigando a expressão algébrica para a condutância, é possível concluir que este novo pico é proveniente da autoenergia de hibridização não diagonal $\tilde{t}(\omega)$ - na vizinhança da singularidade de Van-Hove da densidade de estados, onde o nível de Fermi está localizado - e o estado local do PQ com energia $\epsilon_{0}$, criando condições para a corrente circular através do sistema. Este processo de hibridização, para os parâmetros escolhidos, descrevem o máximo em $\epsilon_{0} \approx-0.5$, como mostraremos na figura. Para o valor menor do acoplamento SO, $\alpha=0.1$, quando $\epsilon_{0} \approx-0.5, C^{\uparrow}$, linha sólida (em vermelho) possui um pico pequeno, enquanto que $C^{\downarrow}$, linha tracejada (em vermelho escuto), tem um pico maior, mostrando os efeitos do acoplamento SO Rashba nesta região. Quando o potencial de porta é reduzido ainda mais, a condutância spin para cima possui um pico já esperado na ressonância, $\epsilon_{0} \approx \epsilon_{F}$, muito maior que o pico em $\epsilon_{0} \approx-0.5$, enquanto esta relação é invertida para a condutância spin para baixo, refletindo o efeito do acoplamento SO Rashba na inversão do spin. Para o regime com forte interação Rashba, $\alpha=0.4$, o pico de $C^{\uparrow}$ próximo ao $\epsilon_{F}$ diminui significativamente e é menor que o pico do $C^{\downarrow}$, linhas tracejadas (em azul) e ponto-tracejadas (em azul escuro), respectivamente.

Uma forma clara de visualizar a performance do dispositivo, é representar a polarização de spin da corrente definida como

$$
p=\frac{C^{\downarrow}-C^{\uparrow}}{C^{\downarrow}+C^{\uparrow}},
$$

a qual é mostrada na figura 4.4 (b). A inversão de spin modifica a polarização da corrente em $\epsilon_{0} \approx-0.3$, linha sólida (em vermelho) para $\alpha=0.1$. Esta polarização prevalece reduzindo o potencial de porta do PQ até $\epsilon_{0} \approx-1$. Nós calculamos a polarização da corrente para outros valores do acoplamento SO Rashba a fim de obter um melhor entendimento de seus efeitos. Na figura 4.4 

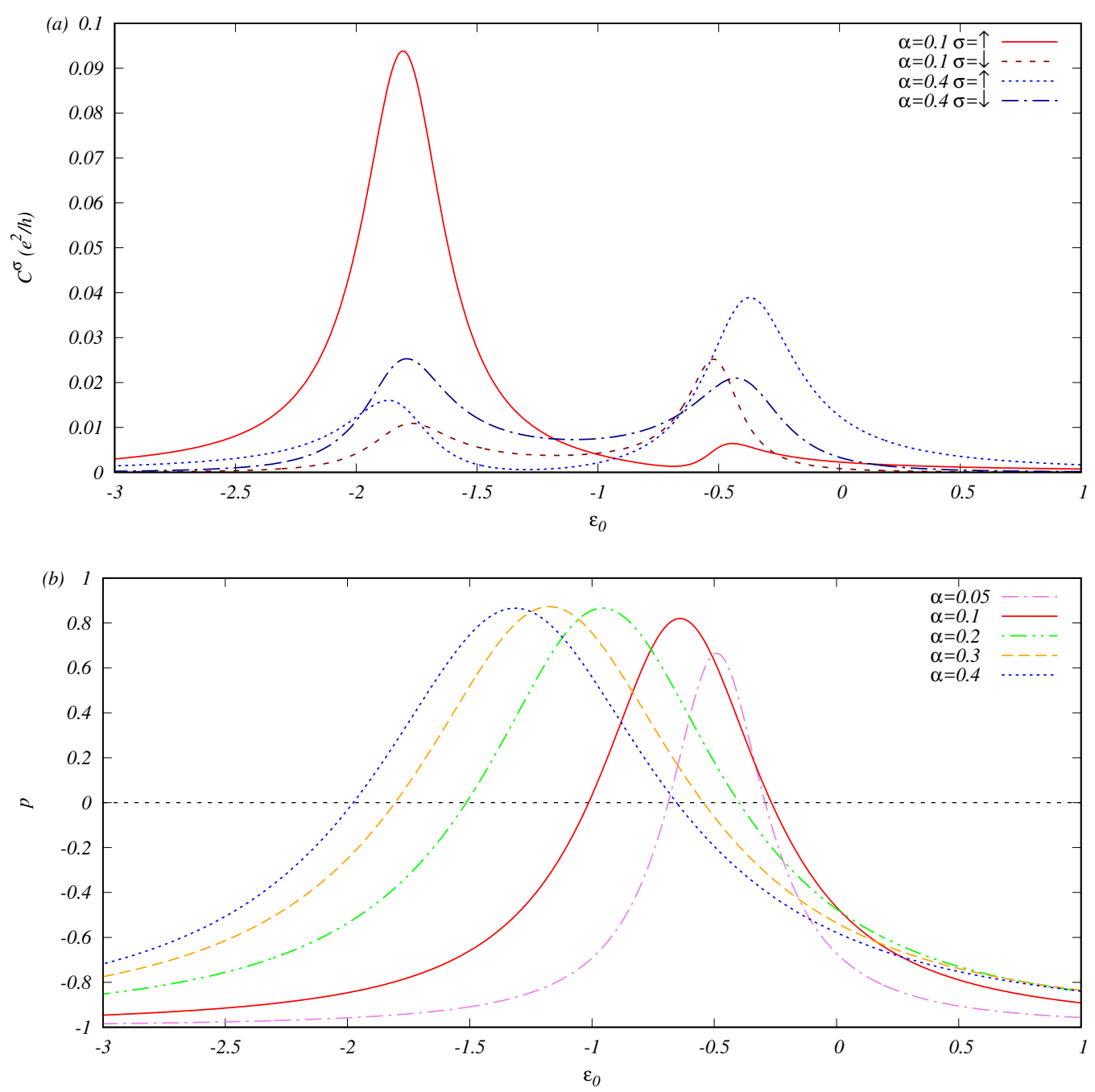

Figura 4.4: (a) Condutância no contato $i=1$ para cada spin, $C^{\sigma}$, e (b) polarização da corrente, $p$, como função do potencial de porta $\epsilon_{0}$, ambos os painéis sem repulsão Coulombiana. No painel (a) para cada spin $\sigma$ e dois valores do acoplamento SO Rashba, $\alpha=0.1$ e $\alpha=0.4$, enquanto no painel (b) para cinco valores de $\alpha, \alpha=0.05,0.1,0.2,0.3$ e 0.4 .

(b) é apresentado a polarização da corrente para $\alpha=0.05, \alpha=0.2, \alpha=0.3$ e $\alpha=0.4$, além do resultado já apresentado para $\alpha=0.1$. É possível concluir que a região $p>0$ é aumentada e o pico em $p$ desloca-se monotonicamente para a direção do $\epsilon_{F}$ à medida que aumentamos o acoplamento SO Rashba. Em particular, para $\alpha=0.4$ a região onde $p>0$ estende-se de $\epsilon_{0} \approx-0.7$ a $\epsilon_{0} \approx-2.0$ como mostrado pela linha pontilhada (em azul) na figura 4.4 (b). Como esperado, este comportamento é consistente com os resultados da figura 4.4 (a).

A conclusão mais importante desta discussão é que o potencial de porta do PQ permite uma perfeita sintonização da polarização da corrente do 
dispositivo, satisfazendo todos os requerimentos para o operação do transistor de spin.

A discussão prévia foi restrita ao problema de um corpo, onde a repulsão Coulombiana no PQ foi negligenciada. Quando $U \neq 0$, a ocupação no $\mathrm{PQ},\left\langle n_{\sigma}\right\rangle$, deve ser calculada autoconsistentemente. Os resultados obtidos para diferentes valores de $U$ são mostrados na figura 4.5 para $\alpha=0.4$. Nós observamos que conforme o potencial de porta $\epsilon_{0}$ é reduzido e o sistema entra no regime de flutuação de carga, $\left\langle n_{\uparrow}\right\rangle$ é maior que $\left\langle n_{\downarrow}\right\rangle$. Contudo, à medida que $\epsilon_{0}$ é reduzido ainda mais, uma inversão ocorre e $\left\langle n_{\downarrow}\right\rangle$ torna-se maior que $\left\langle n_{\uparrow}\right\rangle$ para ambos os valores de $\alpha$, eventualmente atingindo o valor de saturação. A dependência com o spin do número de ocupação reflete o fato de que a simetria de inversão temporal é quebrada devido aos contatos ferromagnéticos e a magnetização da nanofaixa de grafeno. Quando $\epsilon_{0}$ é reduzido, como esperado, um platô com comprimento da ordem de $U$ aparece caracterizando o regime de bloqueamento de Coulomb que não permite a dupla ocupação no PQ. Estas características são mais claramente vistas para $U=0.6$. Finalmente, quando $\epsilon_{0}$ é suficientemente pequeno, o bloqueamento de Coulomb é vencido e a carga por spin se aproxima de seu valor de saturação.

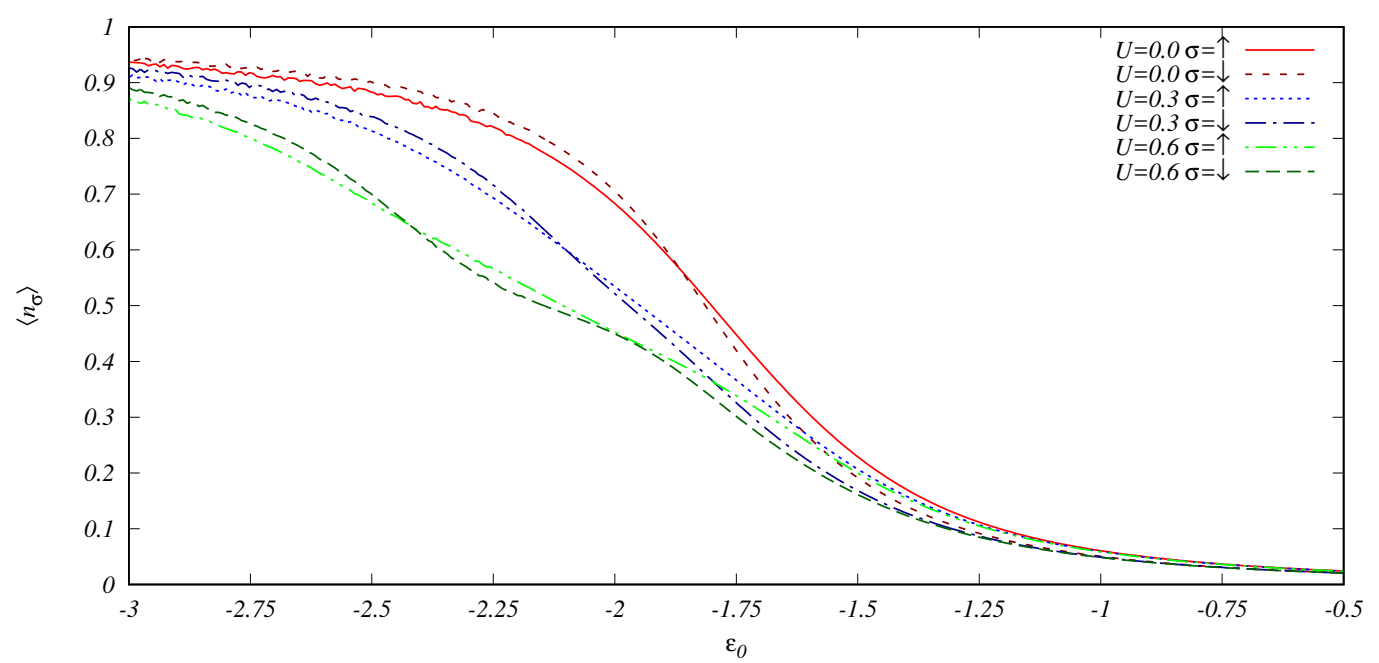

Figura 4.5: Ocupação no PQ, $\left\langle n_{\sigma}\right\rangle$, como função de $\epsilon_{0}$, para $\alpha=0.4$ e vários valores de $U$.

A condutância $C^{\sigma}$ é apresentada na figura 4.6, onde nós podemos observar a separação do pico na vizinhança das ressonâncias $\epsilon_{0}=\epsilon_{F}$ e $\epsilon_{0}=\epsilon_{F}-U$ devido à repulsão Coulombiana. As separações são mais visíveis para $C^{\uparrow}$ e $\alpha=0.1$, mas também estão presentes para $C^{\downarrow}$ e o mesmo $\alpha$, bem como para $\alpha=0.4$. 


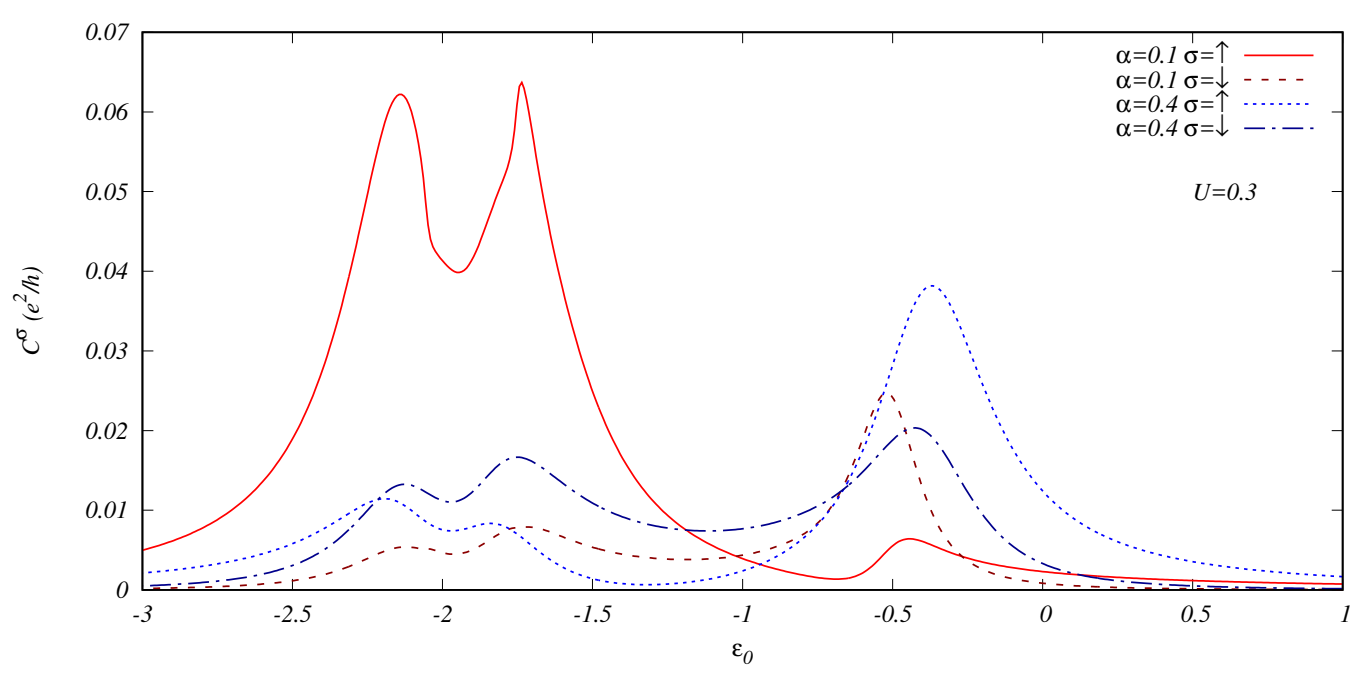

Figura 4.6: Condutância $C^{\sigma}$ para o contato $i=1$ como função do potencial de porta $\epsilon_{0}$, para $\alpha=0.1$ e $\alpha=0.4$.

É importante enfatizar que a altura dos dois picos na condutância quando $U \neq 0$ é menor que o pico da condutância correspondente ao caso com $U=0$ mostrado na figura 4.4 (a). A comparação destas duas situações reflete que o bloqueamento de Coulomb tem efeitos combinados: separa os picos da condutância e reduz sua intensidade. Uma descrição correta da corrente através do PQ na presença de interação elétron-elétron requer ambos ingredientes. Infelizmente, este não é o caso obtido por outros tratamentos, como no caso da aproximação Hubbard I [108]. O pico da condutância em $\epsilon_{0} \approx-0.5$ não apresenta esta separação, devido ao fato que a população eletrônica no PQ é muito baixa, para estes valores do potencial de porta, eliminando o efeito da repulsão Coulombiana local. A corrente flui para este valor de $\epsilon_{0}$, como consequência da hibridização do estado local do PQ e a ressonância ao nível de Fermi da autoenergia $\tilde{t}$ que inverte o spin, como discutido anteriormente. Contudo, a densidade de estados abaixo do nível de Fermi para este valor do potencial de porta é muito baixa, indicando que a carga no PQ é muito pequena. Na figura 4.7 representamos a função $\Im\left\{G_{00}^{\sigma \sigma}(\omega)\right\}$ para $\epsilon_{0}=-0.5$ que claramente exemplifica esta situação. A respeito das propriedades do transistor refletindo a polarização da corrente $p$, a presença da repulsão Coulombiana reduz a simetria do pico e aumenta o alcance da região onde $p>0$ na vizinhança de $\epsilon_{0}=\epsilon_{F}$, como pode ser visto claramente na figura 4.8 (a).

Aumentando o valor da repulsão Coulombiana intra-PQ $U$, a extensão que $p>0$, aumenta, e eventualmente transforma-se em uma região de dois picos, como pode ser visto na figura 4.8 (b), para $U=0.6$. 
Capítulo 4. Corrente de spin polarizada em um ponto quântico conectado a um mar de Fermi com interação spin-órbita

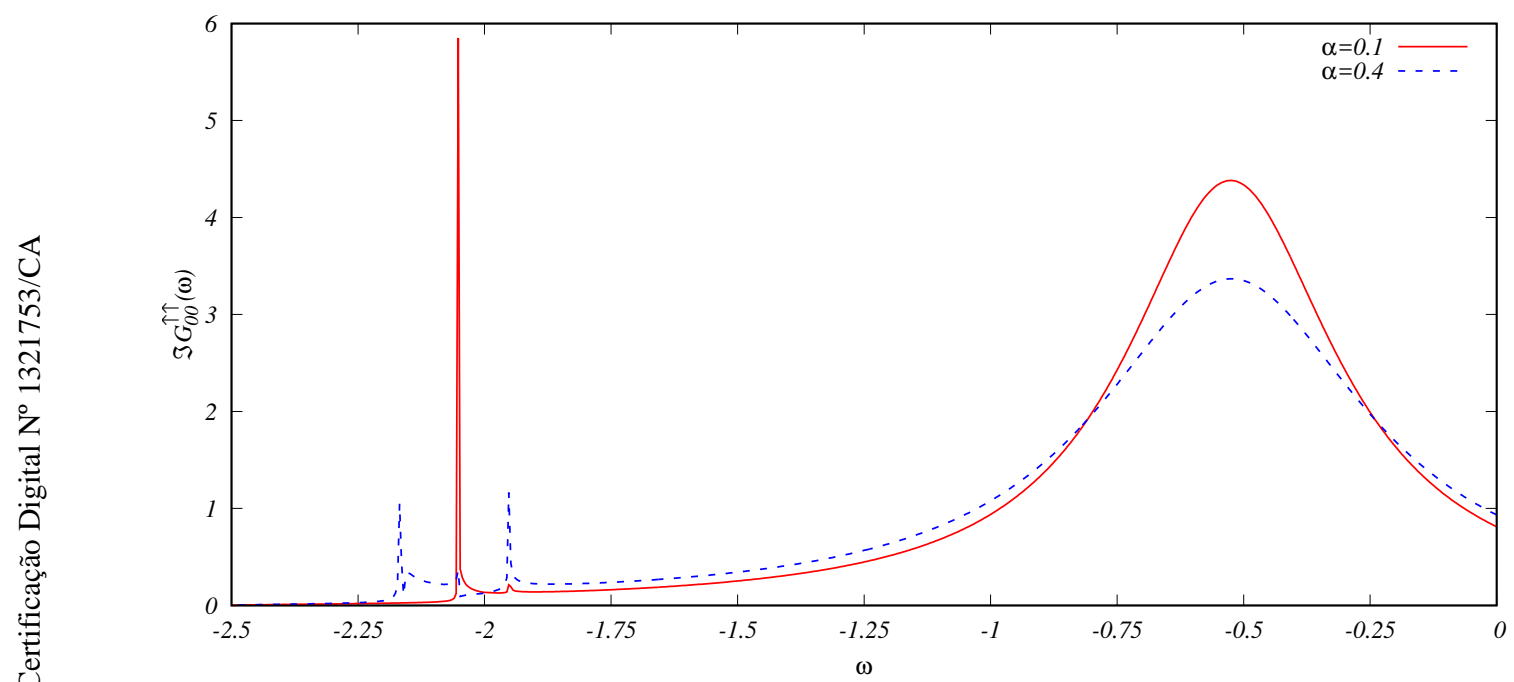

Figura 4.7: Parte imaginária da função de Green diagonal sem repulsão Coulombiana para $\alpha=0.1$ e $\alpha=0.4$. Os valores do potencial de porta e do nível de Fermi são mantidos fixos em $\epsilon_{0}=-0.5$ e $\epsilon_{F}=-1.95$. 
Capítulo 4. Corrente de spin polarizada em um ponto quântico conectado a um mar de Fermi com interação spin-órbita
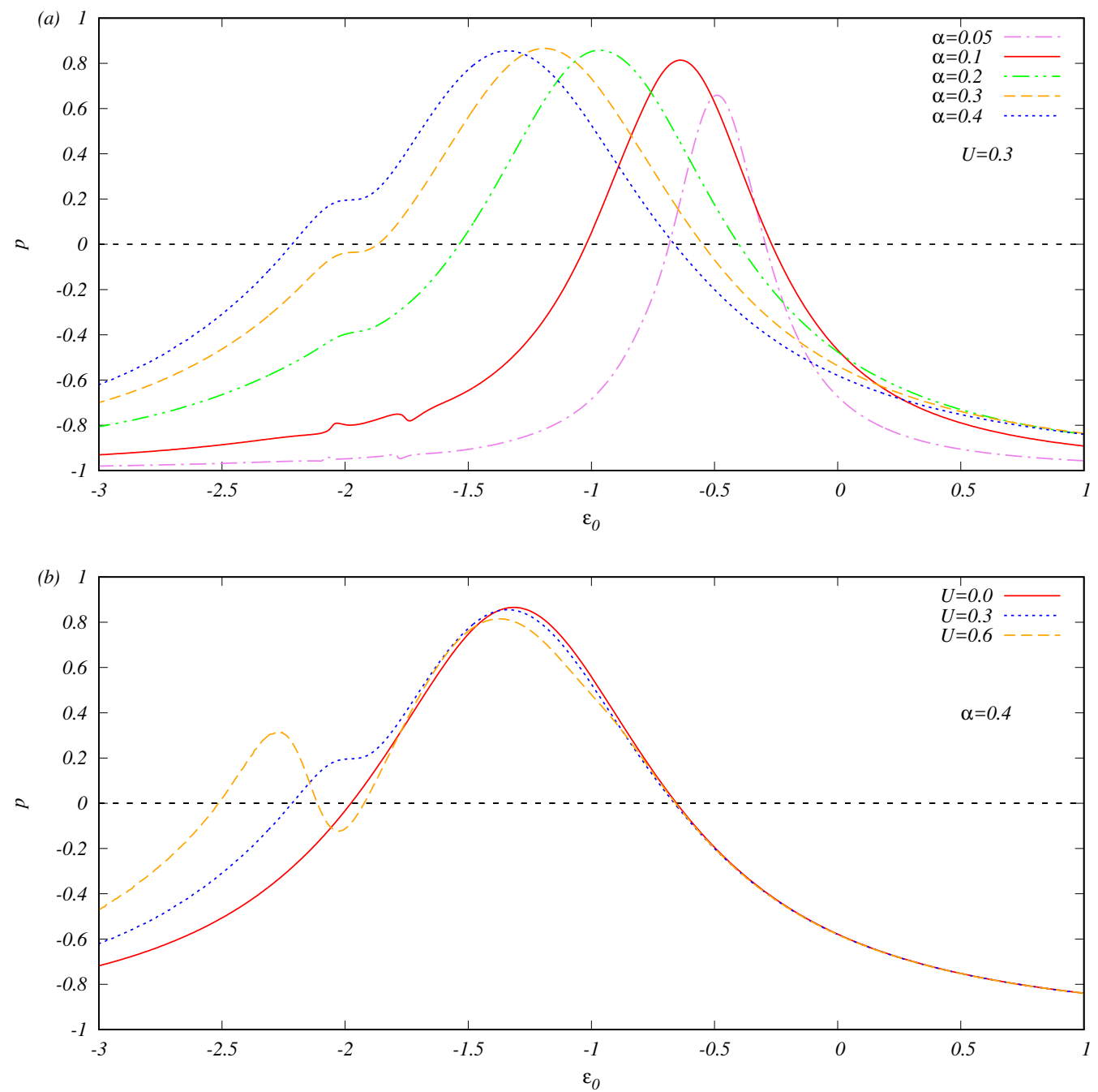

Figura 4.8: (a) Polarização da corrente, $p$, como função do potencial de porta $\epsilon_{0}$ para diferentes $\alpha$, com $U=0.3$, e (b) para $\alpha=0.4$ e três valores de $U$. 
Capítulo 4. Corrente de spin polarizada em um ponto quântico conectado a um mar de Fermi com interação spin-órbita

\subsection{2}

\section{Contatos ferromagnéticos antiparalelos}

Outra configuração possível para detectar a inversão do spin da corrente devido ao acoplamento SO Rashba é conectar o sistema a dois contatos ferromagnéticos com direções de magnetização antiparalelas. Este disposição produz uma corrente de spin que sai do PQ totalmente polarizada na direção oposta à injetada. Neste caso, a corrente no contato $i=1$ é completamente oriunda do processo de inversão de spin que ocorre no PQ. Cabe salientar neste ponto que, como no caso de todas as discussões prévias, nós estamos supondo que os contatos ferromagnéticos são tais que, ao nível de Fermi, existem apenas elétrons com um tipo de spin, consequentemente, a corrente circulando pelo sistema é completamente polarizada, neste caso. A figura 4.9 (a) mostra a condutância spin para baixo, $C^{\downarrow}$, no contato $i=1$ para diferentes valores do acoplamento SO Rashba, para $U=0$. Já que a corrente que circula é exclusivamente oriunda da interação Rashba, para valores pequenos de $\alpha$ a condutância é muito menor que para valores maiores. Nós podemos observar os picos ressonantes em $\epsilon_{0}=-0.5$ e $\epsilon_{0}$ próximo a $\epsilon_{F}$ devido a mesma física já descrita.

Quando $U \neq 0$, figura 4.9 (b) mostra que o pico da condutância, na região do nível de Fermi $\left(\epsilon_{0} \approx \epsilon_{F}\right)$ está desdobrado e sua altura reduzida, como analisado anteriormente para o caso do contato que recebe a corrente ser não magnético. A figura mostra que também neste caso a manipulação de $\epsilon_{0}$ permite controlar a intensidade da condutância. 

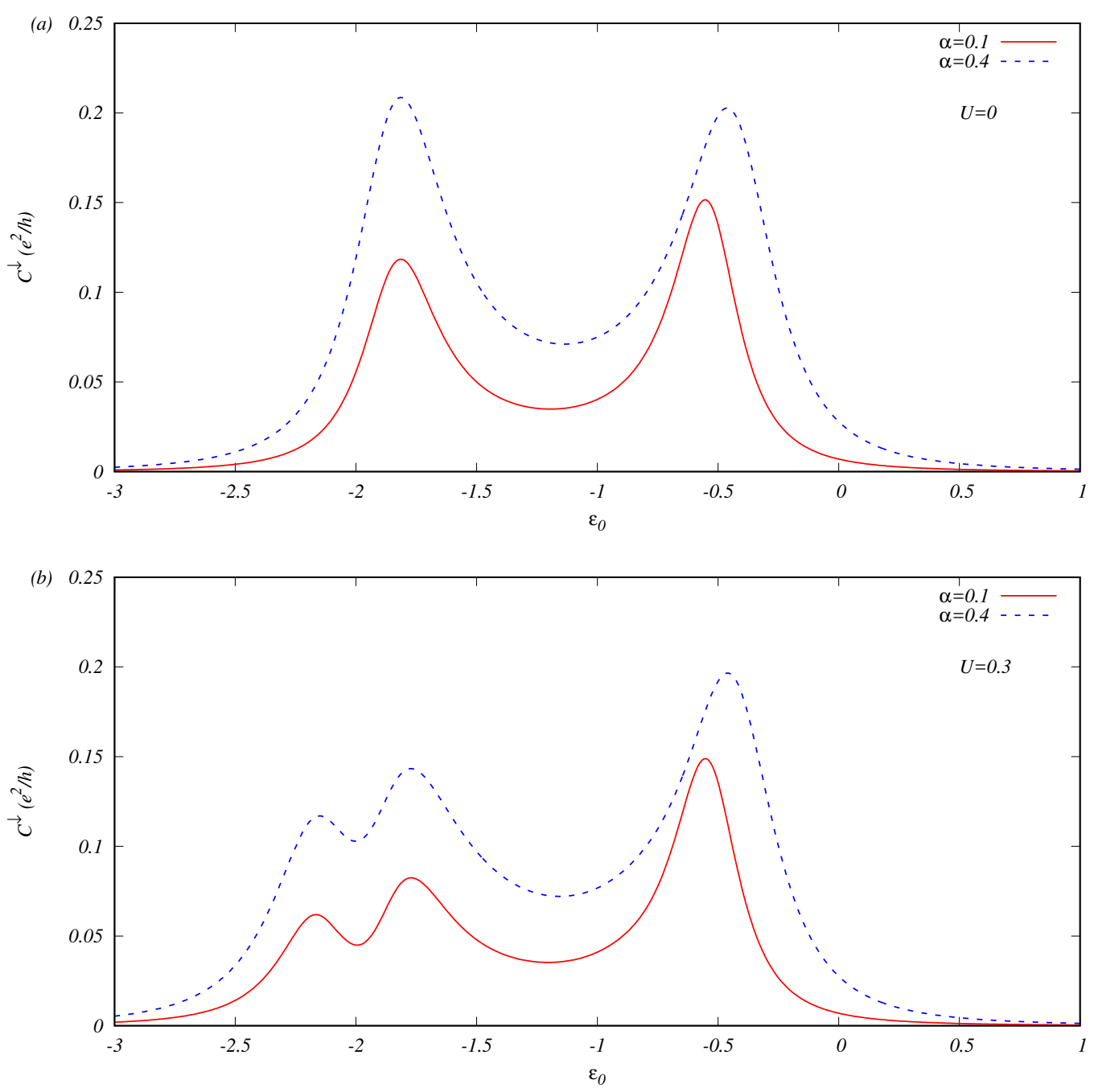

Figura 4.9: Condutância spin para baixo, $C^{\downarrow}$, no contato $i=1$ como função do potencial de porta, $\epsilon_{0}$ para dois valores de $\alpha$. Painel (a) com repulsão Coulombiana $U=0$ e painel (b) com $U=0.3$.

\section{5}

\section{Conclusões e perspectivas futuras}

Um sistema composto por um ponto quântico (PQ) conectado a uma nanofaixa com acoplamento spin-órbita (SO) Rashba, depositado sobre um substrato magnético como seria o caso do níquel $\mathrm{Ni}(111)$ e conectado a dois contatos laterais é estudado na perspectiva de um dispositivo operando como um transistor de spin. Supomos um contato ferromagnético lateral como uma fonte de corrente eletrônica spin polarizada, enquanto a corrente é recolhida por um metal não magnético ou ferromagnético com magnetização antiparalela ao contato que funciona como fonte injetora de elétrons. A conexão do estado local do PQ com o mar de Fermi sobre efeitos da interação SO cria um mecanismo de inversão do spin no PQ, atuando nos elétron que circulam 
no dispositivo. O potencial de porta no PQ comporta-se como um filtro de energia, que permite um controle muito preciso da polarização da corrente que flui através do sistema. Isto mostra que este dispositivo preenche todas as condições necessárias para operar como um transistor de spin.

A repulsão Coulombiana foi incluída no tratamento devido ao confinamento eletrônico dentro do PQ. Ela amplia a região de parâmetros no qual a polarização da corrente pode ser controlada pelo potencial de porta no PQ. Estes aspectos de muitos corpos do problema foram analisados usando a aproximação da analogia da liga Hubbard III, que corretamente descreve as propriedades de transporte do sistema, acima da temperatura Kondo.

Como continuação imediata da análise desse sistema efetuada neste capítulo, destacamos a possibilidade de estudar suas propriedades de transporte quando o sistema se encontra no regime Kondo. É sabido que os picos ressonantes da condutância, localizados ao nível de Fermi $\epsilon_{F}$ e $\epsilon_{F}-U$, formam um único platô quando o sistema encontra-se com uma temperatura inferior a temperatura Kondo devido à aparicão do pico Kondo no nível de Fermi. Assim, a possibilidade de ampliação da região de parâmetros a baixas temperaturas em que o sistema produz uma inversão do spin da corrente circulante pode ser muito interessante. Propomos continuar nossas pesquisas nesta direção. 


\section{5}

\section{Conclusões e perspectivas futuras}

\section{1 \\ Introdução}

Apresentamos nesta tese um estudo teórico do transporte eletrônico em sistemas nanoscópicos e suas propriedades do estado fundamental. Estudo que foi realizado através do formalismo dos operadores de projeção, projector operator approach (POA), e o método das funções de Green.

Ao longo deste último capítulo, sintetizaremos as principais conclusões obtidas no trabalho desenvolvido, bem como as possíveis perspectivas futuras e continuações do trabalho efetuado. As conclusões gerais para a transição $\mathrm{SU}(4)-\mathrm{SU}(2)$ e as propriedades de um filtro de spin do sistema de dois pontos quânticos (PQ) acoplados capacitivamente ocorrerá na seção 5.2. Na seção 5.3, discutiremos as conclusões relativas ao efeito da interação spin-órbita (SO) e na seção 5.4 as referentes ao transistor de spin com interação SO Rashba.

As perspectivas futuras serão mencionadas nas seções 5.5 e 5.6. A primeira devotada ao estudo do efeito Kondo e propriedades magnéticas em sistemas fortemente dependentes da frequência. E na segunda ao estudo da repulsão Coulombiana no volume, extendendo o formalismo de operadores de projeção e usando a filosofia do dynamical mean field theory (DMFT) [116] no espaço das energias.

\section{2}

\section{Sistema de dois pontos quânticos acoplados capacitivamente}

O primeiro sistema analisado, capítulo 2, consiste em dois pontos quânticos (PQ) acoplados capacitivamente entre si e conectados a dois contatos metálicos. Inicialmente investigamos a transição entre o efeito Kondo de simetria $\mathrm{SU}(4)$ e o de simetria $\mathrm{SU}(2)$ devido a um campo magnético externo. O estado fundamental apresenta, como uma propriedade emergente, a simetria $\mathrm{SU}(4)$, mesmo na presença de um campo magnético externo finito, que a princípio destruiria esta simetria.

A transição do efeito Kondo de simetria $\mathrm{SU}(4)$ para $\mathrm{SU}(2)$ introduzida por um campo magnético externo foi caracterizada pelo máximo do número 
de ocupação com spin para baixo nos PQs como função do potencial de porta. Mostramos que, no regime Kondo, o desdobramento Zeeman é uma função universal do potencial de porta em que ocorre o máximo da ocupação com spin para baixo nos PQs. Esta universalidade é perdida conforme o sistema aproxima-se do regime de flutuação de carga, apesar da ocorrência do máximo do número de ocupação com spin para baixo continuar a existir.

Quando o sistema possui uma temperatura Kondo na região de simetria $\mathrm{SU}(2)$, porém próximo à região com simetria $\mathrm{SU}(4)$, a ocupação nos PQs é altamente polarizada pelo campo magnético aplicado aos PQs. Para o caso do GaAs, a polarização pode ser conseguida para campos magnéticos próximos a 0.1 Tesla e a temperatura Kondo da ordem de alguns graus Kelvin.

O sistema foi estudado por dois formalismos diferentes, o projector operator approach (POA) e o mean-field slave bosons approximation (MFSBA), que descreveram as propriedades do estado fundamental com resultados qualitativamente equivalentes. No caso do POA, foi necessário estender o formalismo a análise de duas impurezas acopladas capacitivamente, uma vez que ele foi originalmente desenvolvido para o estudo de apenas uma impureza.

A partir do cálculo da condutância, vemos que este sistema possui excelentes propriedades para operar como um filtro de spin muito eficiente, com possível relevância na spintrônica.

\section{3 \\ Interação spin-órbita}

No capítulo 3 discutimos a influência da interação spin-órbita (SO) Rashba e Dresselhaus em uma cadeia linear infinita. Calculamos a forma da relação de dispersão com ambas as interações SO e apresentamos uma base em que o Hamiltoniano de uma cadeia linear infinita com interação SO Rashba e Dresselhaus é diagonal.

A partir da relação de dispersão foi possível obter a densidade de estados para a cadeia linear infinita com interação SO Rashba e Dresselhaus. Além disso, a relação de dispersão possibilitou o estudo de um dispositivo transistor de spin proposto no capítulo seguinte, capítulo 4, onde utilizamos a interação SO Rashba para inverter o spin do elétron que circula no dispositivo.

Através de uma combinação simétrica e antissimétrica, verificamos como uma impureza se acopla a cadeia linear infinita com interação SO. Observamos que dada uma combinação da impureza, ela se acopla aos elétrons com $k<0$ e uma helicidade e na região com $k>0$ a outra helicidade.

No caso particular de somente interação SO Rashba, verificamos que a interação SO Rashba no volume junto à expressão de Haldane para a 
temperatura Kondo, diminui a temperatura Kondo. Se estiver presente a interação SO Rashba na conexão entre a impureza e o mar de Fermi, vemos que o efeito Kondo é destruído.

Os cálculos apresentados neste capítulo foram todos analíticos, e permitiram obter a influência da interação SO sobre o efeito Kondo.

Como perspectivas futuras no curto prazo temos, em um primeiro momento, a análise do sistema com somente interação SO Dresselhaus e depois a generalização para o sistema com interação SO Rashba e Dresselhaus tanto no volume como no acoplamento da impureza com o mar de Fermi.

\section{4}

\section{Transistor de spin}

Um dispositivo que opera como um transistor de spin foi proposto no capítulo 4. O dispositivo consiste em uma nanofaixa com acoplamento spinórbita (SO) Rashba depositada sobre um substrato de níquel e conectada a dois contatos metálicos laterais. A corrente é injetada no sistema por um contato ferromagnético que atua como uma fonte de elétrons com spin polarizado, ao passo que a corrente é recolhida por outro contato, que pode ser um metal não magnético ou ferromagnético com magnetização antiparalela ao contato que injeta a corrente no sistema.

A conexão do mar de Fermi com interação SO Rashba com o PQ cria um mecanismo de inversão do spin no PQ que atua sobre os elétrons que circulam no sistema. Um filtro de energia é possível através do potencial de porta no $\mathrm{PQ}$, permitindo um controle muito acurado da polarização da corrente que flui pelo sistema. Este dispositivo proposto preenche todas as condições necessárias para operar como um transistor de spin.

Realizamos o tratamento adequado da repulsão Coulombiana no PQ, devido ao confinamento eletrônico nele, com os aspectos de muitos corpos analisados na aproximação da analogia da liga Hubbard III, que descreve corretamente as propriedades de transporte do sistema acima da temperatura Kondo.

Destacamos como continuação imediata da análise desse sistema estudar suas propriedades de transporte quando o sistema se encontra no regime Kondo, com possibilidade de ampliação da região de parâmetros em que o sistema produz uma inversão do spin da corrente circulante. 


\section{5}

\section{Sistemas fortemente dependentes da frequência}

Uma física muito interessante associada ao efeito Kondo é introduzida quando o sistema apresenta uma densidade de estados eletrônicos altamente dependente da frequência próximo ao nível de Fermi.

Alguns autores argumentam que a susceptibilidade magnética da impureza, $\chi_{i m p}$, pode ser negativa [82, 117-120], em particular quando o nível de Fermi está próximo a uma singularidade de Van-Hove para o sistema de uma rede quadrada [119]. Contudo, outros autores defendem que o comportamento observado para $\chi_{i m p}$ não é físico e que as propriedades magnéticas da impureza devem ser deduzidas da susceptibilidade magnética local, $\chi_{l o c}$, [121], sugerindo que o sistema é sempre um líquido de Fermi.

Também pretende-se estudar sistemas mais complexos, como uma impureza em contato com um material supercondutor e um PQ embebido em grafeno, entre outros.

Utilizando o POA para resolver o Hamiltoniano de Anderson, na figura 5.1 apresentamos a $\chi_{i m p}$ como função do potencial de porta para o sistema de uma impureza conectada a uma cadeia linear infinita. Observamos que a $\chi_{i m p}$ muda de sinal a medida que o potencial de porta é reduzido. Os resultados estão em unidades de $t$ e nível de Fermi $\epsilon_{F}$ é fixado próximo a singularidade de Van-Hove, em $-2 t$.

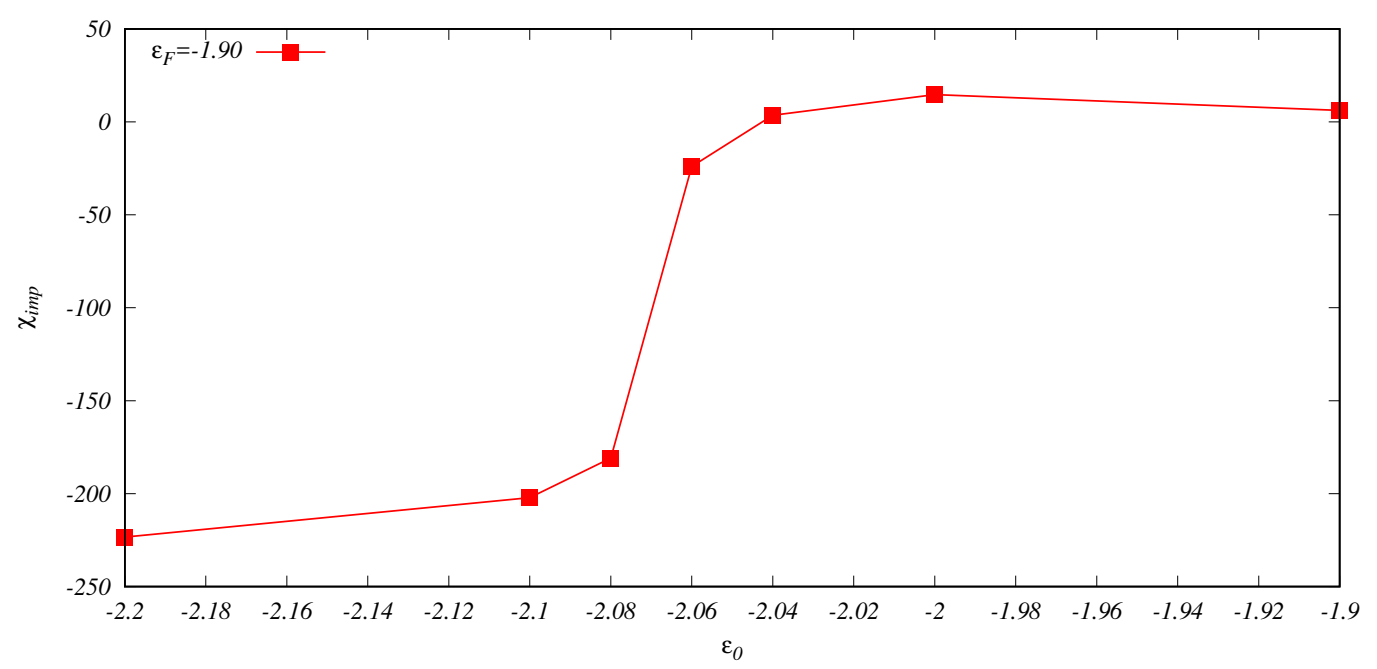

Figura 5.1: Susceptibilidade magnética da impureza $\chi_{i m p}$ como função do elemento diagonal da impureza $\epsilon_{0}$. O nível de Fermi está localizado em $\epsilon_{F}=-1.9$ e $V=0.25$. Resultados em unidades de $t$. 


\section{6 \\ Estudo da repulsão Coulombiana no volume}

\subsection{1}

\section{Descrição do sistema e do formalismo utilizado}

Sistemas com os efeitos da repulsão Coulombiana em todos os sítios da rede são descritos pelo Hamiltoniano de Hubbard [108],

$$
H=\sum_{i, \sigma} \epsilon_{i \sigma} n_{i \sigma}+\sum_{i j} V_{i j}\left(c_{i \sigma}^{\dagger} c_{j \sigma}+c_{j \sigma}^{\dagger} c_{i \sigma}\right)+U \sum_{i} n_{i \uparrow} n_{i \downarrow} .
$$

É possível encontrar uma solução exata quando o sistema possui dimensão infinita ou com grande número de coordenação. A solução é obtida por meio do dynamical mean field theory (DMFT) [116], que permite realizar um mapeamento do sistema descrito pelo Hamiltoniano de Hubbard num descrito pelo Hamiltoniano de uma impureza de Anderson [7]. Este mapeamento substitui, por meio de um cálculo autoconsistente, o modelo da rede pelo de uma impureza embebida em um meio efetivo.

Uma excelente aproximação ao estudo de sistemas reais consiste em impor esse mapeamento, apesar de sistemas com infinitas dimensões não serem reais. Mapeamento que permite entender as propriedades de um sistema com a repulsão Coulombiana local no volume a partir da solução do problema equivalente do Hamiltoniano de Anderson.

Este formalismo exige o conhecimento das funções de Green [38]. Entretanto, estamos propondo estender a filosofia do DMFT, originalmente desenvolvido no espaço das frequências, ao cálculo da energia do estado fundamental no espaço das energias. Destacamos como sistema de relevância para este cálculo os descritos por uma rede de Bethe.

\section{6 .2}

\section{Extensão do formalismo do DMFT ao espaço das energias}

Ao longo dos próximos parágrafos, desenvolveremos um formalismo baseado em sucessivas renormalizações do elemento diagonal do Hamiltoniano usando a filosofia do DMFT. Entretanto, em vez de trabalhar no espaço das frequências, como usualmente o DMFT é empregado, tal formalismo ocorrerá no espaço das energias.

No Hamiltoniano de Anderson, a energia do estado fundamental é caracterizada pelo elemento de matriz do sítio da impureza, $\epsilon_{0}$, a repulsão Coulombiana $U$ entre dois elétrons neste sítio e o elemento diagonal $\epsilon$ de todos os outros sítios da rede. Escreveremos a energia do estado fundamental como função destes parâmetros genericamente como $E\left(\epsilon_{0}, U ; \epsilon\right)$. 
A influência da repulsão Coulombiana na impureza será descrita por um Hamiltoniano de um corpo equivalente com o elemento diagonal renormalizado, $\tilde{\epsilon}^{(1)}$, e a energia do estado fundamental $E\left(\tilde{\epsilon}^{(1)} ; \epsilon\right)$. O índice $i$ em $\tilde{\epsilon}^{(i)}$ refere-se ao número de renormalizações ou iterações do elemento diagonal.

Agora substituiremos o elemento diagonal de todos os sítios da rede, a menos do sítio da impureza, por $\tilde{\epsilon}^{(1)}$ e efetuaremos uma nova renormalização. Este procedimento denota a incorporação do efeito da repulsão Coulombiana na impureza a todos os sítios da rede. A energia do estado fundamental é agora caracterizada por $E\left(\epsilon_{0}, U ; \tilde{\epsilon}^{(1)}\right)$. Realizando uma nova renormalização, a energia é dada por $E\left(\tilde{\epsilon}^{(2)} ; \tilde{\epsilon}^{(1)}\right)$.

Após a n-éssima renormalização, a energia do sistema ficará $E\left(\tilde{\epsilon}^{(n+1)} ; \tilde{\epsilon}^{(n)}\right)$. Tomando o limite de $n$ tendendo a infinito, a energia resulta ser $E\left(\tilde{\epsilon}^{(n)} ; \tilde{\epsilon}^{(n)}\right)$, onde a informação da repulsão $U$ em todos os sítios da rede está incluída através do elemento diagonal $\tilde{\epsilon}^{(n)}$. Este processo permitirá o cálculo da energia do sistema com $U$ em todos os sítios impondo,

$$
E\left(\epsilon_{0}, U ; \tilde{\epsilon}\right)=E(\tilde{\epsilon} ; \tilde{\epsilon})
$$

onde omitimos o índice $n$.

Efetuando as renormalizações sistematicamente, construímos um mapeamento do Hamiltoniano da impureza de Anderson no Hamiltoniano de Hubbard, porque a repulsão Coulombiana que inicialmente estava restrita a um único sítio é considerada nas renormalizações de todos os sítios da rede.

O processo descrito junto à equação (5-2) constituem as ideias centrais do formalismo proposto, o qual consiste em estender a filosofia do DMFT ao espaço das energias para calcular a energia do estado fundamental do Hamiltoniano de Hubbard. Este formalismo é aplicado tentativamente para um sistema de dois sítios e dois elétrons e outro de três sítios e três elétrons. $\mathrm{O}$ resultado é comparado com os resultados exatos para estes sistemas simples.

\subsection{3}

\section{Sistema de dois sítios e dois elétrons}

A repulsão Coulombiana $U$ no sistema de dois sítios introduz uma interação antiferromagnética entre os spins dos elétrons, que, como consequência, fará com que a solução esteja no espaço de Hilbert de spin total zero.

Escolhemos este sistema por sua simplicidade e função didática de como opera o formalismo. Ele pode ser resolvido por uma série de abordagens diferentes com relativa facilidade; calculando exatamente os autovalores do Hamiltoniano através sua matriz, utilizando o DMFT em sua forma tradicional a partir das funções de Green e finalmente adotando o formalismo que estamos 
propondo no espaço das energias junto à abordagem dos operadores de projeção (POA). Estes resultados poderão ser comparados entre si.

O Hamiltoniano de Anderson para este sistema é,

$$
H=\sum_{\sigma} V\left(c_{0 \sigma}^{\dagger} c_{1 \sigma}+c_{1 \sigma}^{\dagger} c_{0 \sigma}\right)+\sum_{\sigma} \epsilon_{1} n_{1 \sigma}+\sum_{\sigma} \epsilon_{0} n_{0 \sigma}+U n_{0 \uparrow} n_{0 \downarrow},
$$

onde os índices 0 e 1 referem-se ao sítio da impureza e ao outro sítio sem repulsão Coulombiana $U$, respectivamente. $c_{i \sigma}^{\dagger}\left(c_{i \sigma}\right)$ é o operador de criação (aniquilação) de um elétron no sítio $i=0$ ou 1 com energia local $\epsilon_{i} \mathrm{e}$ $n_{i \sigma}=c_{i \sigma}^{\dagger} c_{i \sigma}$.

Apresentaremos inicialmente a solução exata deste problema escrevendo uma matriz que contenha todas as configurações possíveis de spin total nulo. A base para o espaço de Hilbert deste sistema singleto é formada pelos vetores $|\alpha\rangle$, com dois elétrons no primeiro sítio, $|\beta\rangle$, com dois elétrons no segundo sítio, e $|\gamma\rangle$, a combinação antissimétrica de um elétron em cada sítio, definidos como:

$$
\begin{gathered}
|\alpha\rangle \equiv|\uparrow \downarrow, 0\rangle, \\
|\beta\rangle \equiv|0, \uparrow \downarrow\rangle \\
\text { e }|\gamma\rangle \equiv \frac{1}{\sqrt{2}}(|\uparrow, \downarrow\rangle-|\downarrow, \uparrow\rangle) .
\end{gathered}
$$

Podemos escrever o estado fundamental como uma combinação linear de $|\alpha\rangle,|\beta\rangle$ e $|\gamma\rangle$. A matriz do Hamiltoniano nesta base apresenta a forma,

$$
H=\begin{gathered}
|\alpha\rangle \\
\langle\alpha| \\
\langle\beta| \\
\langle\gamma|
\end{gathered}\left(\begin{array}{ccc}
2 \epsilon_{0}+U & 0 & |\beta\rangle \\
0 & 2 \epsilon_{1}+U & \sqrt{2} V \\
\sqrt{2} V & \sqrt{2} V & \epsilon_{0}+\epsilon_{1}
\end{array}\right),
$$

onde $V$ é o termo não diagonal que conecta os dois sítios e $\epsilon_{0}$ e $\epsilon_{1}$ são as energias locais dos elétrons nos sítios 0 e 1 , respectivamente.

Adotamos, por simplicidade, o elemento diagonal dos sítios $\epsilon_{0}=\epsilon_{1}=0$, de modo que a energia do estado fundamental $E$ resulta em

$$
E=\frac{U-\sqrt{U^{2}+16 V^{2}}}{2},
$$

onde escolhemos como solução a do autovalor de menor energia.

Também podemos encontrar a energia do estado fundamental usando o POA. Escolhemos como subespaço $S_{1}$ o composto unicamente pelo estado com cada sítio com um elétron. Como o estado fundamental deve ser um singleto, escolhemos $|\gamma\rangle$ que forma parte da base em que o Hamiltoniano está escrito, equação (5-6), como a autofunção que está contida no subespaço $S_{1}$. 
A figura 5.2 ilustra uma representação diagramática do sistema analisado. No diagrama do lado esquerdo da figura temos o sistema observado como um problema de muitos corpos, com repulsão Coulombiana $U$ no sítio com elemento diagonal $\epsilon_{0}$, que está conectado a outro sítio com elemento diagonal $\tilde{\epsilon}$ por meio da conexão $V$. O lado direito apresenta o mesmo sistema descrito como um problema de um corpo, onde a repulsão Coulombiana está inclusa no sistema através do elemento diagonal renormalizado $\tilde{\epsilon}$.

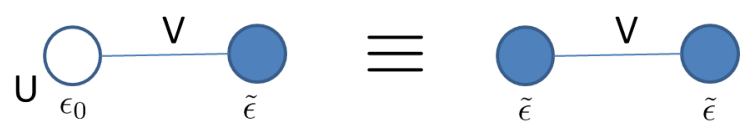

Figura 5.2: Diagrama representando a equivalência do sistema de muitos corpos, na esquerda, com o sistema de um corpo, na direita. Os sítios hachurados possuem repulsão Coulombiana local entre os elétrons no sítio através das renormalizações.

Para o sistema descrito como um problema de muitos corpos temos que,

$$
E=\tilde{\epsilon}+\frac{2 V^{2}}{E-2 \tilde{\epsilon}}+\frac{2 V^{2}}{E-U},
$$

onde utilizamos as equações (A-9) e (A-10) do formalismo POA e, novamente por simplicidade, adotamos o elemento diagonal do sítio com repulsão Coulombiana como nulo.

Por outro lado, após efetuar as renormalizações no espírito do DMFT no espaço das energias, o sistema é descrito por um problema de um corpo. Assim como no problema de muitos corpos, obtemos com o POA a equação

$$
E=2 \tilde{\epsilon}+\frac{4 V^{2}}{E-2 \tilde{\epsilon}} .
$$

A solução do estado fundamental obtida a partir da resolução do sistema de equações expresso nas equações (5-9) e (5-10) é a solução exata do estado fundamental, equação (5-8). Para este sistema simples de dois sítios e dois elétrons, o cálculo realizado indica que utilizar a filosofia do DMFT no espaço das energias acarreta na solução exata do estado fundamental.

Utilizando o DMFT (tradicional) para o cálculo da energia do estado fundamental, é necessário calcular as funções de Green em função da frequência $\omega$. Para o sistema de um corpo podemos escrever a função de Green como

$$
G_{00}(\omega)=\frac{1}{\omega-\tilde{\epsilon}(\omega)-\frac{V^{2}}{\omega-\tilde{\epsilon}(\omega)}} .
$$

Ressaltamos que $\tilde{\epsilon}(\omega)$ é uma função complexa de $\omega$, já que deve satisfazer as propriedades da função de Green. A partir da parte real e imaginária de $G_{00}(\omega)$ obtemos o sistema de equações, 


$$
\Re G_{00}^{-1}(\omega)=\omega-\Re \tilde{\epsilon}-\frac{V^{2}(\omega-\Re \tilde{\epsilon})}{(\omega-\Re \tilde{\epsilon})^{2}+(\Im \tilde{\epsilon})^{2}}
$$

e

$$
\Im G_{00}^{-1}(\omega)=-\Im \tilde{\epsilon}-\frac{V^{2}(\Im \tilde{\epsilon})}{(\omega-\Re \tilde{\epsilon})^{2}+(\Im \tilde{\epsilon})^{2}} .
$$

Já para o problema de muitos corpos, usando o operador resolvente [114] calculamos a função de Green,

$$
\begin{aligned}
G_{00}(\omega) & =\left(\left\langle f\left|c_{0}^{\dagger}(\omega+H-i \eta)^{-1} c_{0}\right| f\right\rangle\right. \\
& \left.+\left\langle f\left|c_{0}(\omega-H-i \eta)^{-1} c_{0}^{\dagger}\right| f\right\rangle\right)
\end{aligned}
$$

onde $|f\rangle$ representa a autofunção do estado fundamental.

A energia do estado fundamental pode ser calculada por,

$$
E=\langle H\rangle=4 V\left\langle c_{0 \uparrow} c_{1 \uparrow}^{\dagger}\right\rangle+2 \epsilon_{0}\left\langle c_{0 \sigma}^{\dagger} c_{0 \sigma}\right\rangle+2 \epsilon_{1}\left\langle c_{1 \sigma}^{\dagger} c_{1 \sigma}\right\rangle+U\left\langle n_{0 \uparrow} n_{0 \downarrow}\right\rangle,
$$

onde usamos o Hamiltoniano da equação 5-3 e $\left\langle c_{0 \sigma}^{\dagger} c_{1 \sigma}\right\rangle=\left\langle c_{1 \sigma}^{\dagger} c_{0 \sigma}\right\rangle$.

A equação de movimento para este sistema é

$$
\left(\omega-\epsilon_{0}\right) G_{00}=1+V G_{10}+U \Gamma_{00},
$$

onde

$$
G_{i j}(\omega)=\ll c_{i \sigma} ; c_{j \sigma}^{\dagger} \gg_{\omega}
$$

e a equação de correlação de três operadores para o sistema de dois sítios

$$
\Gamma_{00}(\omega)=\ll n_{0 \uparrow} c_{0 \downarrow} ; c_{0 \downarrow}^{\dagger} \gg_{\omega} .
$$

Analogamente,

$$
\begin{aligned}
& G_{10}=\frac{V}{\omega-\epsilon_{1}} G_{00} . \\
& G_{11}=\frac{V}{\omega-\epsilon_{1}} G_{01} .
\end{aligned}
$$

A energia do estado fundamental pode finalmente ser calculada por [38],

$$
\langle A B\rangle=\frac{1}{\pi} \int_{-\infty}^{\epsilon_{F}} \Im \ll A ; B \gg_{\omega} d \omega
$$

e as equações (5-15), (5-16), (5-19) e (5-20), onde $A$ e $B$ são dois operadores. Após alguns passos algébricos temos a energia do estado fundamental,

$$
E=\langle H\rangle=\int_{-\infty}^{\epsilon_{F}} \Im\left(\frac{3 V^{2}}{\omega-\epsilon_{1}}+2+\frac{2 V^{2}}{\left(\omega-\epsilon_{1}\right)^{2}}+\frac{1}{\omega-\epsilon_{0}}\right) G_{00}(\omega) d \omega .
$$

Para o problema de muitos corpos, calculamos a $G_{00}(\omega)$ através do operador resolvente, equação (5-14), e $\epsilon_{1}=\tilde{\epsilon}$ e $\epsilon_{0}=0$.

A figura 5.3 apresenta os resultados para o cálculo da energia do estado fundamental $E$ do Hamiltoniano de Hubbard do sistema de dois sítios. A solução é apresentada em função da escala adimensional $U / V$, obtida usando as 
diferentes formas de cálculo.

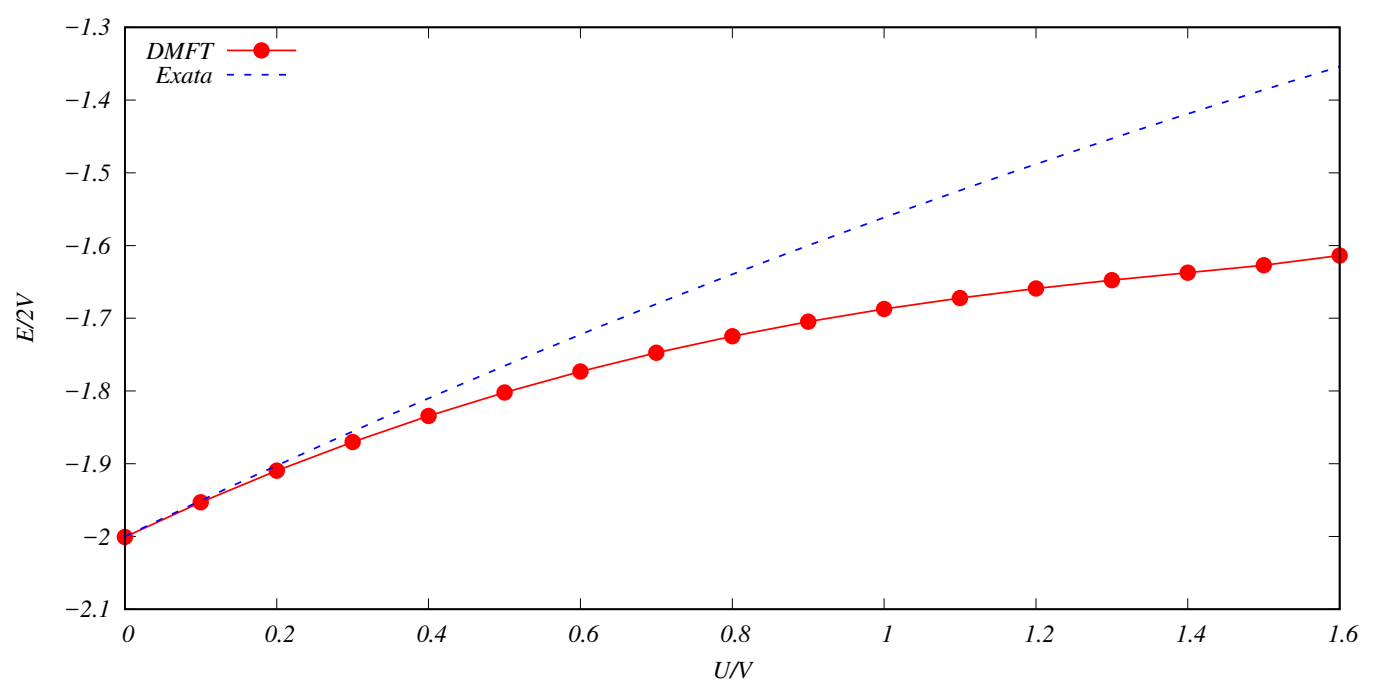

Figura 5.3: O gráfico apresenta a solução da energia do estado fundamental usando diferentes formalismos, o DMFT (círculos e linha contínua vermelha), a solução analítica e exata do sistema (linhas azul tracejada), obtida pelos autovalores do Hamiltoniano e pelo POA junto a filosofia do DMFT no espaço das energias. O eixo y está normalizado pelo valor da energia quando $U=0$.

Como mostramos, a energia do estado fundamental usando a filosofia do DMFT no espaço das energias e o POA é exata. No mesmo gráfico representamos a solução da equação (5-8), o novo método que propomos e que coincide com a solução exata e a solução usando o DMFT (tradicional, no espaço das frequências). Para este último resultado vemos que, quando a razão $U / V$ tende a zero, converge para a solução exata. Contudo, quando a razão aumenta e afasta-se de zero, o DMFT fornece um resultado cuja energia resulta ser inferior à exata.

No limite $U \rightarrow \infty$, somente será permitido que um elétron ocupe cada sítio, já que a ocupação dupla não será possível, consequentemente os elétrons não podem se deslocar e cada sítio estará preenchido. Neste limite, a energia do sistema vai assintoticamente a zero.

\subsection{4}

\section{Sistema de três sítios e três elétrons}

Com a solução do sistema de dois sítios com dois elétrons e spin total nulo, o sistema com três sítios e três elétrons apresenta-se como um sistema um pouco mais complexo, que, contrariamente ao anterior, não possui spin total nulo. 
Propomos o estudo deste sistema já que o resultado anterior pelo formalismo proposto foi exato e não ocorreu o mesmo com o DMFT. Gostaríamos de determinar quão eficiente é o formalismo proposto. A fim de esclarecer este aspecto, analisamos o sistema de três sítios com três elétrons, que apesar de mais complexo, ainda pode ser resolvido de forma exata.

Para representar o Hamiltoniano, uma base para o espaço de Hilbert deste problema que inclua o estado fundamental, assim como no caso do sistema de dois sítios e dois elétrons, é composta pelas nove autofunções a seguir,

$$
\begin{aligned}
& |\uparrow, \downarrow, \uparrow\rangle,|\downarrow, \uparrow, \uparrow\rangle,|\uparrow, \uparrow, \downarrow\rangle, \\
& |\uparrow \downarrow, \uparrow, 0\rangle,|\uparrow \downarrow, 0, \uparrow\rangle,|\uparrow, \uparrow \downarrow, 0\rangle,|0, \uparrow \downarrow, \uparrow\rangle,|\uparrow, 0, \uparrow \downarrow\rangle \text { e }|0, \uparrow, \uparrow \downarrow\rangle .
\end{aligned}
$$

O Hamiltoniano de Hubbard fica nesta base como,

$$
\left(\begin{array}{ccccccccc}
0 & 0 & 0 & 0 & V & -V & V & -V & 0 \\
0 & 0 & 0 & V & -V & 0 & -V & 0 & V \\
0 & 0 & 0 & -V & 0 & V & 0 & V & -V \\
0 & V & -V & U & V & -V & 0 & 0 & 0 \\
V & -V & 0 & V & U & 0 & 0 & -V & 0 \\
-V & 0 & V & -V & 0 & U & V & 0 & 0 \\
V & -V & 0 & 0 & 0 & V & U & 0 & -V \\
-V & 0 & V & 0 & -V & 0 & 0 & U & V \\
0 & V & -V & 0 & 0 & 0 & -V & V & U
\end{array}\right)
$$

O polinômio característico desta matriz é uma equação do nono grau,

$$
\begin{aligned}
& -36 U^{4} V^{4} \lambda-12 U^{5} V^{2} \lambda^{2}+180 U^{3} V^{4} \lambda^{2}-U^{6} \lambda^{3}+66 U^{4} V^{2} \lambda^{3}-333 U^{2} V^{4} \lambda^{3} \\
& +6 U^{5} \lambda^{4}-144 U^{3} V^{2} \lambda^{4}+270 U V^{4} \lambda^{4}-15 U^{4} \lambda^{5}+156 U^{2} V^{2} \lambda^{5}-81 V^{4} \lambda^{5} \\
& +20 U^{3} \lambda^{6}-84 U V^{2} \lambda^{6}-15 U^{2} \lambda^{7}+18 V^{2} \lambda^{7}+6 U \lambda^{8}-\lambda^{9}=0
\end{aligned}
$$

cuja solução de $\lambda$ fornece os autovalores da matriz.

Dentre as nove raízes possíveis, a de relevância ao nosso problema com os limites adequados para a energia do estado fundamental é

$$
\begin{aligned}
E & =\Re\left[\frac{2 U}{3}+\frac{(1+i \sqrt{3})\left(-U^{2}-27 V^{2}\right)}{6\left(-U^{3}+9 \sqrt{-U^{4} V^{2}-27 U^{2} V^{4}-243 V^{6}}\right)^{\frac{1}{3}}}\right. \\
& \left.-\frac{1}{6}(1-i \sqrt{3})\left(-U^{3}+9 \sqrt{-U^{4} V^{2}-27 U^{2} V^{4}-243 V^{6}}\right)^{\frac{1}{3}}\right] .
\end{aligned}
$$

Apesar da solução em notação complexa, verificamos numericamente que sua parte imaginária é nula. Também lembramos que a matriz da equação (5-25) 
é hermitiana, e que a equação (5-26) é uma forma conveniente de escrever a solução. Dividindo $E$ por $V$, observamos que a solução depende somente da razão entre $U / V$.

Assim como no sistema anterior, calculamos a energia do estado fundamental do sistema utilizando a filosofia do DMFT no espaço das energias e o POA. Precisamos de duas equações, uma originária do problema de um corpo e outra do problema de muitos corpos, conforme o diagrama na figura 5.4.

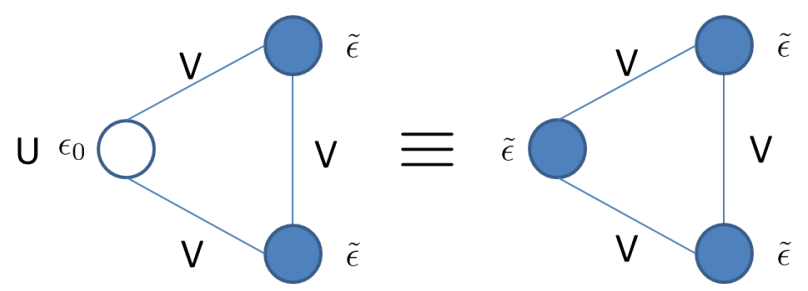

Figura 5.4: No lado esquerdo temos o sistema de muitos corpos e no direito o sistema de um corpo.

É conveniente efetuar uma combinação simétrica e antissimétrica dos vetores dos estados do sítio 2 e do sítio 3 (figura 5.5),

$$
|1\rangle,|+\rangle=\frac{|2\rangle+|3\rangle}{\sqrt{2}} \mathrm{e}|-\rangle=\frac{|2\rangle-|3\rangle}{\sqrt{2}} .
$$

Com esta combinação, podemos representar o sistema pelo diagrama apresentado na figura 5.5.

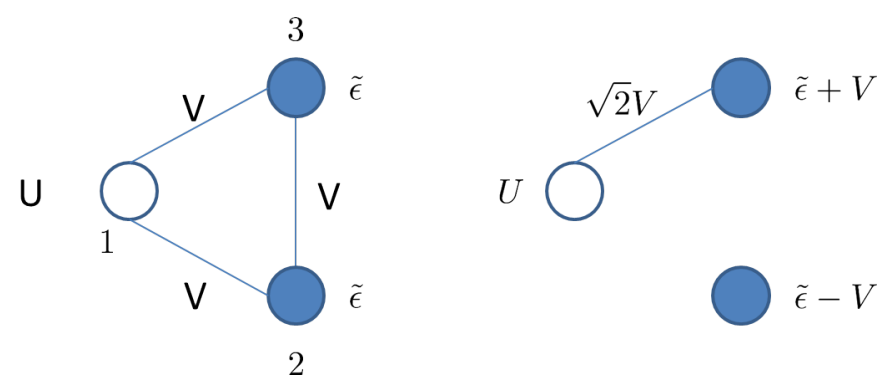

Figura 5.5: No lado esquerdo, o sistema antes da combinação simétrica antisimétrica e, no direito, o sistema após a combinação.

Utilizamos o POA com o subespaço $S_{1}$ com um elétron em cada sítio, obtendo do sistema de um corpo,

$$
E=3 \tilde{\epsilon}+\frac{4 V^{2}}{E-(3 \tilde{\epsilon}-V)}+\frac{4 V^{2}}{E-(3 \tilde{\epsilon}+V)} .
$$

Para o sistema de muitos corpos, escolhemos o subespaço $S_{1}$ aquele com um elétron na impureza e dois elétrons no sítios com termo diagonal $\tilde{\epsilon}+V$, obtendo,

$$
E=2(\tilde{\epsilon}+V)+\frac{2 V^{2}}{E-(\tilde{\epsilon}+V+U)} .
$$


A solução com os casos limites corretos, de ir a zero quando $U \rightarrow \infty$ e $E=-3 V$ quando $U \rightarrow 0$ é:

$$
E=\frac{3}{4}\left(U-\sqrt{U^{2}+16 V^{2}}\right)
$$

que é uma função de $U / V$.

Na figura 5.6, temos a comparação da solução do POA na filosofia do DMFT no espaço das energias, equação (5-30), com a solução exata do sistema, equação (5-26), e a solução numérica, calculando os autovalores da matriz na equação (5-24) computacionalmente.

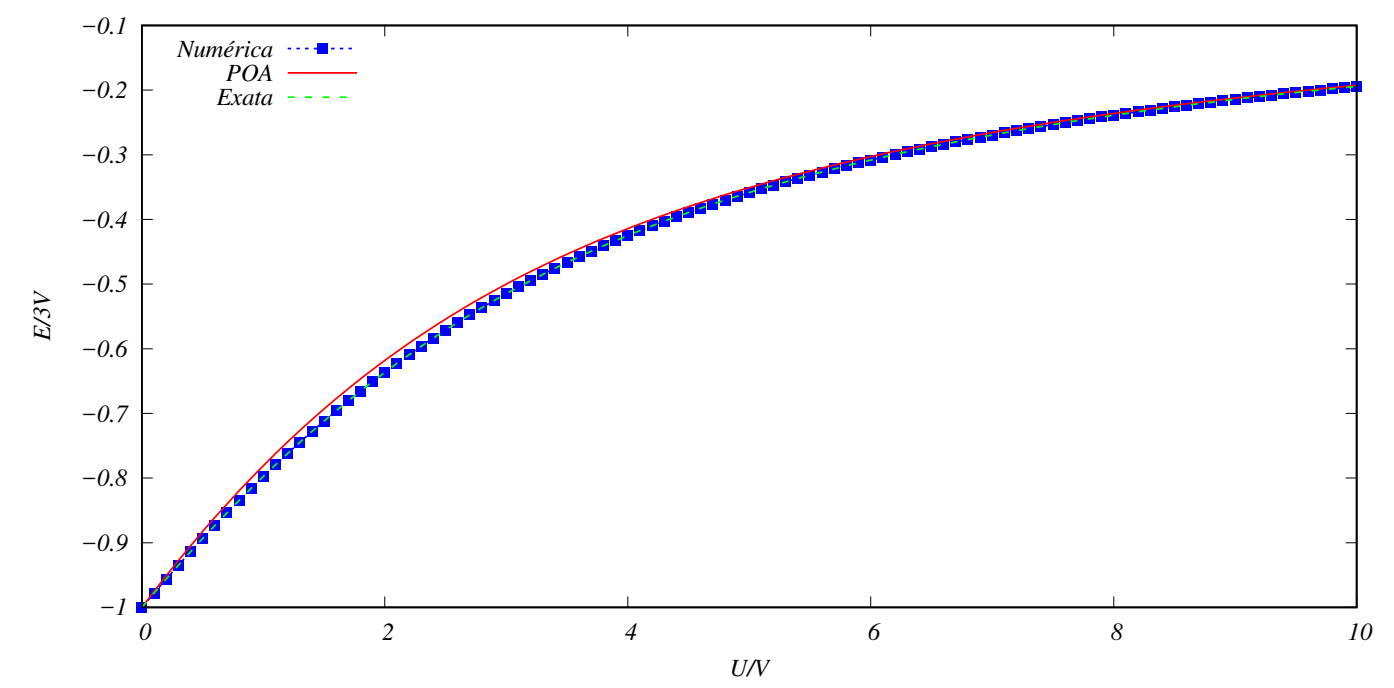

Figura 5.6: Energia do estado fundamental para o sistema de três sítios e três elétrons. Apresentamos a solução da filosofia do DMFT no espaço das energias e POA (linha contínua vermelha), equação (5-30), a solução analítica exata (linha tracejada verde), equação (5-26), e a solução numérica dos autovalores da matriz na equação (5-24) (quadrados azuis). O eixo y está normalizado pelo valor da energia quando $U=0$.

Observando o gráfico vemos que embora o resultado não pareça exato para o formalismo proposto como no problema de dois sítios com dois elétrons, ele está em excelente acordo com a solução exata. O resultado desvia-se ligeiramente da solução em torno e $U / V=2$. A medida que a razão $U / V$ distancia-se da região $U / V=2$, seja em direção a zero ou infinito, as curvas tendem a coincidir.

O formalismo do espírito do DMFT no espaço das energias aliado ao POA apresenta-se como uma solução muito mais simples que o DMFT (tradicional, no espaço das frequências), o qual exige o cálculo das funções de Green. O comportamento nos limites da repulsão Coulombiana tendendo a zero ou infinito estão corretos, desviando-se levemente na região próxima a $U / V=2$. 


\section{Referências bibliográficas}

[1] MADELUNG, O.. Introduction to Solid-State Theory. New York, Springer, 1981.

[2] VAN DELFT, D.; KES, P.. The discovery of superconductivity. Physics Today, 63 (9): 38-43, 2010.

[3] KAMERLingH-ONNES, H.. Comm. Phys. Lab. Univ. Leiden, Nov. 1911. Suppl. 29.

[4] FRANCK, J. P.; MANCHESTER, F. D. ; MARTIN, D. L.. The specific heat of pure copper and of some dilute copper+iron alloys showing a minimum in the electrical resistance at low temperatures. Proceedings of the Royal Society of London A: Mathematical, Physical and Engineering Sciences, 263 (1315): 494-507, 1961.

[5] PHILLIPS, P.. Advanced Solid State Physics. Cambridge University Press, 2 edition, 2012.

[6] SARACHIK, M. P.; CORENZWIT, E. ; LONGINOTTI, L. D.. Resistivity of mo-nb and mo-re alloys containing $1 \%$ Fe. Phys. Rev., 135: A1041-A1045, Aug 1964.

[7] HEWSON, A. C.. The Kondo Problem to Heavy Fermions. Cambridge University Press, 1993.

[8] KONDO, J.. Resistance minimum in dilute magnetic alloys. Progress of Theoretical Physics, 32 (1): 37, 1964.

[9] NAGAMUNE, Y.; SAKAKI, H.; KOUWENHOVEN, L. P.; MUR, L. C.; HARMANS, C. J. P. M.; MOTOHISA, J. ; NOGE, H.. Single electron transport and current quantization in a novel quantum dot structure. Applied Physics Letters, 64 (18): 2379-2381, 1994.

[10] GOLDHABER-GORDON, D.; SHTRIKMAN, H.; MAHALU, D.; ABUSCHMAGDER, D.; MEIRAV, U. ; KASTNER, M.. Kondo effect in a singleelectron transistor. Nature, 391 (6663): 156-159, 1998. 
[11] HORVATIĆ, B.; SOKCEVIĆ, D. ; ZLATIĆ, V.. Finite-temperature spectral density for the Anderson model. Phys. Rev. B, 36: 675-683, Jul 1987.

[12] LOPES, V.; PADILlA, R. A.; MARTINS, G. B. ; ANDA, E. V.. SU(4)$\mathrm{SU}(2)$ crossover and spin-filter properties of a double quantum dot nanosystem. Phys. Rev. B, 95: 245133, Jun 2017.

[13] ROURA-BAS, P.; TOSI, L.; ALIGIA, A. A. ; HALLBERG, K.. Interplay between quantum interference and Kondo effects in nonequilibrium transport through nanoscopic systems. Phys. Rev. B, 84: 073406, Aug 2011.

[14] TOSI, L.; ROURA-BAS, P. ; ALIGIA, A.. Transition between SU(4) and SU(2) Kondo effect. Physica B: Condensed Matter, 407 (16): 3259 - 3262, 2012. Frontiers of Condensed Matter V (FCM 2010).

[15] KOUWENHOVEN, L.; GLAZMAN, L.. Revival of the Kondo effect. Physics World, 14 (1): 33, 2001.

[16] ANDERSON, P. W.. A poor man's derivation of scaling laws for the Kondo problem. Journal of Physics C: Solid State Physics, 3 (12): 2436, 1970.

[17] WILSON, K. G.. The renormalization group: Critical phenomena and the Kondo problem. Rev. Mod. Phys., 47: 773-840, Oct 1975.

[18] HEEGER, A.. Localized moments and nonmoments in metals: The Kondo effect. volumen 23 de Solid State Physics, p. $283-411$. Academic Press, 1970.

[19] KOUWENHOVEN, L.; MARCUS, C.. Quantum dots. Physics World, 11 (6): 35, 1998.

[20] KOUWENHOVEN, L. P.; MARCUS, C. M.; MCEUEN, P. L.; TARUCHA, S.; WESTERVELT, R. M. ; WINGREEN, N. S.. Electron Transport in Quantum Dots, p. 105-214. Springer Netherlands, Dordrecht, 1997.

[21] SCHRIEFFER, J. R.; WOLFF, P. A.. Relation between the Anderson and Kondo hamiltonians. Phys. Rev., 149: 491-492, Sep 1966.

[22] ANDA, E. V.; CHIAPPE, G.; BÜSSER, C. A.; DAVIDOVICH, M. A.; MARTINS, G. B.; HEIDRICH-MEISNER, F. ; DAGOTTO, E.. Method 
to study highly correlated nanostructures: The logarithmicdiscretization embedded-cluster approximation. Phys. Rev. B, 78: 085308, Aug 2008.

[23] HEIDRICH-MEISNER, F.; FEIGUIN, A. E. ; DAGOTTO, E.. Real-time simulations of nonequilibrium transport in the single-impurity Anderson model. Phys. Rev. B, 79: 235336, Jun 2009.

[24] ROURA-BAS, P.; HAMAD, I. J. ; ANDA, E. V.. Ground state of the impurity Anderson model revisited: A projector operator solution. Phys. Status Solidi (b), 252 (2): 421-430, 2015.

[25] HAMAD, I. J.; ROURA-BAS, P.; ALIGIA, A. A. ; ANDA, E. V.. Selfconsistent hybridization expansions for static properties of the Anderson impurity model. Phys. Status Solidi (b), 253 (3): 478-485, 2016.

[26] DA SILVA, V. L.. Um formalismo de operadores de projeção para resolver o Hamiltoniano de Anderson. Dissertação de mestrado, Departamento de Física, Pontifícia Universidade Católica do Rio de Janeiro (PUC-Rio), Rua Marquês de São Vicente, 225, Gávea - Rio de Janeiro, RJ - Brasil, 92013.

[27] NEWNS, D. M.; HEWSON, A. C.. A local Fermi liquid theory of intermediate valence systems. Journal of Physics F: Metal Physics, 10 (11): 2429, 1980.

[28] KOTLIAR, G.; RUCKENSTEIN, A. E.. New functional integral approach to strongly correlated Fermi systems: The Gutzwiller approximation as a saddle point. Phys. Rev. Lett., 57: 1362-1365, Sep 1986.

[29] DORIN, V.; SCHLOTTMANN, P.. Slave-boson mean-field theory of the Anderson lattice with finite $U$ and orbital degeneracy. Phys. Rev. B, 47: 5095-5105, Mar 1993.

[30] DONG, B.; LEI, X. L.. Kondo-type transport through a quantum dot under magnetic fields. Phys. Rev. B, 63: 235306, May 2001.

[31] DONG, B.; LEI, X. L.. Kondo-type transport through a quantum dot: a new finite-U slave-boson mean-field approach. J. Phys. Condens. Matter, 13 (41): 9245, 2001. 
[32] DONG, B.; LEI, X. L.. Kondo effect and antiferromagnetic correlation in transport through tunneling-coupled double quantum dots. Phys. Rev. B, 65: 241304, May 2002.

[33] DONG, B.; LEI, X. L.. Nonequilibrium Kondo effect in a multilevel quantum dot near the singlet-triplet transition. Phys. Rev. B, 66: 113310, Sep 2002.

[34] WINGREEN, N. S.; MEIR, Y.. Anderson model out of equilibrium: Noncrossing-approximation approach to transport through a quantum dot. Phys. Rev. B, 49: 11040-11052, Apr 1994.

[35] BICKERS, N. E.. Review of techniques in the large- $n$ expansion for dilute magnetic alloys. Rev. Mod. Phys., 59: 845-939, Oct 1987.

[36] TOSI, L.; ROURA-BAS, P.; LLOIS, A. M. ; MANUEL, L. O.. Effects of vertex corrections on diagrammatic approximations applied to the study of transport through a quantum dot. Phys. Rev. B, 83: 073301, Feb 2011.

[37] SCHOTTE, K.; SCHOTTE, U.. Interpretation of Kondo experiments in a magnetic field. Physics Letters A, 55 (1): 38 - 40, 1975.

[38] ZUBAREV, D. N.. Double-time Green functions in statistical physics. Phys. Usp., 3 (3): 320, 1960.

[39] BETHE, H.. Zur theorie der metalle. Zeitschrift für Physik, 71 (3): 205-226, Mar 1931.

[40] HUBBARD, J.. Electron correlations in narrow energy bands III. An improved solution. Proceedings of the Royal Society of London A: Mathematical, Physical and Engineering Sciences, 281 (1386): 401-419, 1964.

[41] HALDANE, F. D. M.. Scaling theory of the asymmetric Anderson model. Phys. Rev. Lett., 40: 416-419, Feb 1978.

[42] HALDANE, F. D. M.. Scaling theory of the asymmetric Anderson model. Phys. Rev. Lett., 40: 911-911, Mar 1978.

[43] TETTAMANZI, G. C.; VERDUIJN, J.; LANSBERGEN, G. P.; BLAAUBOER, M.; CALDERÓN, M. J.; AGUADO, R. ; ROGGE, S.. Magnetic-field probing of an SU(4) Kondo resonance in a single-atom transistor. Phys. Rev. Lett., 108: 046803, Jan 2012. 
[44] JARILLO-HERRERO, P.; KONG, J.; VAN DER ZANT, H. S. J.; DEKKER, C.; KOUWENHOVEN, L. P. ; DE FRANCESCHI, S.. Orbital Kondo effect in carbon nanotubes. Nature, 434 (7032): 484-488, Mar 2005.

[45] HOLLEITNER, A. W.; CHUDNOVSKIY, A.; PFANNKUCHE, D.; EBERL, K. ; BLICK, R. H.. Pseudospin Kondo correlations versus hybridized molecular states in double quantum dots. Phys. Rev. B, 70: 075204, Aug 2004.

[46] LIM, J. S.; CHOI, M.-S.; CHOI, M. Y.; LÓPEZ, R. ; AGUADO, R.. Kondo effects in carbon nanotubes: From SU(4) to SU(2) symmetry. Phys. Rev. B, 74: 205119, Nov 2006.

[47] TOSI, L.; ROURA-BAS, P. ; ALIGIA, A. A.. Orbital Kondo spectroscopy in a double quantum dot system. Phys. Rev. B, 88: 235427, Dec 2013.

[48] KELLER, A. J.; AMASHA, S.; WEYMANN, I.; MOCA, C. P.; RAU, I. G.; KATINE, J. A.; SHTRIKMAN, H.; ZARAND, G. ; GOLDHABER-GORDON, D.. Emergent SU(4) Kondo physics in a spin-charge-entangled double quantum dot. Nat Phys, 10 (2): 145-150, Feb 2014. Article.

[49] NISHIKAWA, Y.; CURTIN, O. J.; HEWSON, A. C.; CROW, D. J. G. ; BAUER, J.. Conditions for observing emergent SU(4) symmetry in a double quantum dot. Phys. Rev. B, 93: 235115, Jun 2016.

[50] NISHIKAWA, Y.; HEWSON, A. C.; CROW, D. J. G. ; BAUER, J.. Analysis of low-energy response and possible emergent SU(4) Kondo state in a double quantum dot. Phys. Rev. B, 88: 245130, Dec 2013.

[51] BÜSSER, C. A.; FEIGUIN, A. E. ; MARTINS, G. B.. Electrostatic control over polarized currents through the spin-orbital Kondo effect. Phys. Rev. B, 85: 241310, Jun 2012.

[52] BÜSSER, C. A.; VERNEK, E.; ORELLANA, P.; LARA, G. A.; KIM, E. H.; FEIGUIN, A. E.; ANDA, E. V. ; MARTINS, G. B.. Transport in carbon nanotubes: Two-level $\mathrm{SU}(2)$ regime reveals subtle competition between Kondo and intermediate valence states. Phys. Rev. B, 83: 125404, Mar 2011.

[53] VERNEK, E.; BÜSSER, C. A.; ANDA, E. V.; FEIGUIN, A. E. ; MARTINS, G. B.. Spin filtering in a double quantum dot device: Numerical renormalization group study of the internal structure of the Kondo state. Applied Physics Letters, 104 (13): 132401, 2014. 
[54] SASAKI, S.; TAMURA, H.; AKAZAKI, T. ; FUJISAWA, T.. Fano-kondo interplay in a side-coupled double quantum dot. Phys. Rev. Lett., 103: 266806, Dec 2009.

[55] JARILLO-HERRERO, P.; KONG, J.; VAN DER ZANT, H.; DEKKER, C.; KOUWENHOVEN, L. ; DE FRANCESCHI, S.. Orbital Kondo effect in carbon nanotubes. Nature, 434 (7032): 484-488, MAR 242005.

[56] LAIRD, E. A.; KUEMMETH, F.; STEELE, G. A.; GROVE-RASMUSSEN, K.; NYGARD, J.; FLENSBERG, K. ; KOUWENHOVEN, L. P.. Quantum transport in carbon nanotubes. Rev. Mod. Phys., 87 (3): 703-764, JUL 282015.

[57] ANDERSON, P. W.. Localized magnetic states in metals. Phys. Rev., 124: 41-53, Oct 1961.

[58] NEWNS, D.; READ, N.. Mean-field theory of intermediate valence/heavy fermion systems. Advances in Physics, 36 (6): 799-849, 1987.

[59] FULDE, P.. Electron Correlations in Molecules and Solids. Springer-Verlag, third edition, 2013.

[60] RECHER, P.; SUKHORUKOV, E. V. ; LOSS, D.. Quantum dot as spin filter and spin memory. Phys. Rev. Lett., 85: 1962-1965, Aug 2000.

[61] BORDA, L.; ZARÁND, G.; HOFSTETTER, W.; HALPERIN, B. I. ; VON DELFT, J.. SU(4) Fermi liquid state and spin filtering in a double quantum dot system. Phys. Rev. Lett., 90: 026602, Jan 2003.

[62] FEINBERG, D.; SIMON, P.. Splitting electronic spins with a Kondo double dot device. Applied Physics Letters, 85 (10): 1846-1848, 2004.

[63] HANSON, R.; VANDERSYPEN, L. M. K.; VAN BEVEREN, L. H. W.; ELZERMAN, J. M.; VINK, I. T. ; KOUWENHOVEN, L. P.. Semiconductor few-electron quantum dot operated as a bipolar spin filter. Phys. Rev. B, 70: 241304, Dec 2004.

[64] DAHLHAUS, J. P.; MAIER, S. ; KOMNIK, A.. Spin-polarized current generation and detection by a double quantum dot structure. Phys. Rev. B, 81: 075110, Feb 2010.

[65] MIRELES, F.; ULLOA, S. E.; ROJAS, F. ; COTA, E.. Bipolar spin filter in a quantum dot molecule. Applied Physics Letters, 88 (9): 093118, 2006. 
[66] HEDIN, E. R.; JOE, Y. S.. Sensitive spin-polarization effects in an Aharonov-Bohm double quantum dot ring. Journal of Applied Physics, 110 (2): 026107, 2011.

[67] LANDAUER, R.; BÜTTIKER, M.. Resistance of small metallic loops. Phys. Rev. Lett., 54: 2049-2052, May 1985.

[68] MEIR, Y.; WINGREEN, N. S.. Landauer formula for the current through an interacting electron region. Phys. Rev. Lett., 68: 25122515, Apr 1992.

[69] KELDYSH, L. V.. Diagram technique for nonequilibrium processes. Zh. Eksp. Teor. Fiz., 47: 1515-1527, 1964. [Sov. Phys. JETP20, 1018 (1965)].

[70] MANCHON, A.; KOO, H. C.; NITTA, J.; FROLOV, S. M. ; DUINE, R. A.. New perspectives for Rashba spin-orbit coupling. Nat Mater, 14 (9): 871-882, Sep 2015. Review.

[71] DRESSELHAUS, G.. Spin-orbit coupling effects in zinc blende structures. Phys. Rev., 100: 580-586, Oct 1955.

[72] BYCHKOV, Y. A.; RASHBA, E. I.. Oscillatory effects and the magnetic susceptibility of carriers in inversion layers. Journal of Physics C: Solid State Physics, 17 (33): 6039, 1984.

[73] VAS'KO, F.. Spin splitting in the spectrum of two-dimensional electrons due to the surface potential. JETP Letters, 30: 541-544, Nov 1979. P. Zh. Eksp. Teor. Fiz. 30, 574-577 (1979).

[74] BYCHKOV, Y. A.; RASHBA, E. I.. Properties of a 2d electron gas with lifted spectral degeneracy. JETP Letters, 39: 78-81, Jan 1984. P. Zh. Eksp. Teor. Fiz. 39, 66-69 (1984).

[75] DATTA, S.; DAS, B.. Electronic analog of the electro-optic modulator. Applied Physics Letters, 56 (7): 665-667, 1990.

[76] MALECKI, J.. The two dimensional Kondo model with Rashba spin-orbit coupling. Journal of Statistical Physics, 129 (4): 741-757, Nov 2007.

[77] ŽITKO, R.; BONČA, J.. Kondo effect in the presence of Rashba spin-orbit interaction. Phys. Rev. B, 84: 193411, Nov 2011. 
[78] ZAREA, M.; ULLOA, S. E. ; SANDLER, N.. Enhancement of the Kondo effect through Rashba spin-orbit interactions. Phys. Rev. Lett., 108: 046601, Jan 2012.

[79] ISAEV, L.; AGTERBERG, D. F. ; VEKHTER, I.. Kondo effect in the presence of spin-orbit coupling. Phys. Rev. B, 85: 081107, Feb 2012.

[80] KIKOIN, K.; AVISHAI, Y.. Interplay between Kondo tunneling and Rashba precession. Phys. Rev. B, 86: 155129, Oct 2012.

[81] GRAP, S.; MEDEN, V. ; ANDERGASSEN, S.. Interplay of Coulomb interaction and spin-orbit effects in multilevel quantum dots. Phys. Rev. B, 86: 035143, Jul 2012.

[82] MASTROGIUSEPPE, D.; WONG, A.; INGERSENT, K.; ULLOA, S. E. ; SANDLER, N.. Kondo effect in graphene with Rashba spin-orbit coupling. Phys. Rev. B, 90: 035426, Jul 2014.

[83] WONG, A.; ULLOA, S. E.; SANDLER, N. ; INGERSENT, K.. Influence of Rashba spin-orbit coupling on the Kondo effect. Phys. Rev. B, 93: 075148, Feb 2016.

[84] DE SOUSA, G. R.; SILVA, J. F. ; VERNEK, E.. Kondo effect in a quantum wire with spin-orbit coupling. Phys. Rev. B, 94: 125115, Sep 2016.

[85] GAINON, D.; HEEGER, A. J.. Suppression of the Kondo many-body scattering effect. Phys. Rev. Lett., 22: 1420-1423, Jun 1969.

[86] MEIR, Y.; WINGREEN, N. S.. Spin-orbit scattering and the Kondo effect. Phys. Rev. B, 50: 4947-4950, Aug 1994.

[87] MIRELES, F.; KIRCZENOW, G.. Ballistic spin-polarized transport and Rashba spin precession in semiconductor nanowires. Phys. Rev. B, 64: 024426, Jun 2001.

[88] CHEN, L.; HAN, R.-S.. Kondo temperature of Anderson impurity model in a quantum wire with spin-orbit coupling. ArXiv e-prints, Nov. 2017. arXiv:1711.05505.

[89] VAN WEPEREN, I.; TARASINSKI, B.; EELTINK, D.; PRIBIAG, V. S.; PLISSARD, S. R.; BAKKERS, E. P. A. M.; KOUWENHOVEN, L. P. ; WIMMER, M.. Spin-orbit interaction in InSb nanowires. Phys. Rev. B, 91: 201413, May 2015. 
[90] HASAN, M. Z.; KANE, C. L.. Colloquium: Topological insulators. Rev. Mod. Phys., 82: 3045-3067, Nov 2010.

[91] AWSCHALOM, D. D.; FLATTE, M. E.. Challenges for semiconductor spintronics. Nat Phys, 3 (3): 153-159, Mar 2007.

[92] PRINZ, G. A.. Magnetoelectronics. Science, 282 (5394): 1660-1663, 1998.

[93] WOLF, S. A.; AWSCHALOM, D. D.; BUHRMAN, R. A.; DAUGHTON, J. M.; VON MOLNÁR, S.; ROUKES, M. L.; CHTCHELKANOVA, A. Y. ; TREGER, D. M.. Spintronics: A spin-based electronics vision for the future. Science, 294 (5546): 1488-1495, 2001.

[94] ŽUTIĆ, I.; FABIAN, J. ; DAS SARMA, S.. Spintronics: Fundamentals and applications. Rev. Mod. Phys., 76: 323-410, Apr 2004.

[95] CHAPPERT, C.; FERT, A. ; VAN DAU, F. N.. The emergence of spin electronics in data storage. Nat Mater, 6 (11): 813-823, Nov 2007.

[96] FABIAN, J.; MATOS-ABIAGUE, A.; ERTLER, C.; STANO, P. ; ZUTIC, I.. Semiconductor spintronics. Acta Phys. Slovaca, 57: 565, Aug,Oct 2007.

[97] LOSS, D.; DIVINCENZO, D. P.. Quantum computation with quantum dots. Phys. Rev. A, 57: 120-126, Jan 1998.

[98] NITTA, J.; AKAZAKI, T.; TAKAYANAGI, H. ; ENOKI, T.. Gate control of spin-orbit interaction in an inverted $\mathrm{In}_{0.53} \mathrm{Ga}_{0.47} \mathrm{As} / \mathrm{In}_{0.52} \mathrm{Al}_{0.48} \mathrm{As}$ heterostructure. Phys. Rev. Lett., 78: 1335-1338, Feb 1997.

[99] ANDERSON, P. W.. Localized magnetic states in metals. Phys. Rev., 124: 41-53, Oct 1961.

[100] MAGDA, G. Z.; JIN, X.; HAGYMÁSI, I.; VANCSÓ, P.; OSVÁTH, Z.; NEMES-INCZE, P.; HWANG, C.; BIRÓ, L. P. ; TAPASZTÓ, L.. Room-temperature magnetic order on zigzag edges of narrow graphene nanoribbons. Nature, 514: 608-611, Oct 2014.

[101] DEDKOV, Y. S.; FONIN, M.; RÜDIGER, U. ; LAUBSCHAT, C.. Rashba effect in the graphene/Ni(111) system. Phys. Rev. Lett., 100: 107602, Mar 2008. 
[102] SUN, Q.-F.; WANG, J. ; GUO, H.. Quantum transport theory for nanostructures with Rashba spin-orbital interaction. Phys. Rev. B, 71: 165310, Apr 2005.

[103] WANG, D.-K.; CHENG, S.-G.. Spin-polarized transport in ferromagnet/Rashba quantum dot/ferromagnet system. Physics Letters $A$, 365 (3): $235-239,2007$.

[104] SOUZA, F. M.; JAUHO, A. P. ; EGUES, J. C.. Spin-polarized current and shot noise in the presence of spin flip in a quantum dot via nonequilibrium green's functions. Phys. Rev. B, 78: 155303, Oct 2008.

[105] MEYER, D.; NOLTING, W.. Ferromagnetism in the periodic Anderson model. a comparison of spectral density approximation (SDA), modified alloy analogy (MAA) and modified perturbation theory (MPT). Eur. Phys. J. B, 18 (3): 385-395, 2000.

[106] JUNGWIRTH, T.; WUNDERLICH, J. ; OLEJNIK, K.. Spin hall effect devices. Nat Mater, 11 (5): 382-390, May 2012.

[107] OYARZÚN, S.; NANDY, A. K.; RORTAIS, F.; ROJAS-SÁNCHEZ, J.-C.; DAU, M.-T.; NOËL, P.; LACZKOWSKI, P.; POUGET, S.; OKUNO, H.; VILA, L.; VERGNAUD, C.; BEIGNÉ, C.; MARTY, A.; ATTANÉ, J.-P.; GAMBARELLI, S.; GEORGE, J.-M.; JAFFRÈS, H.; BLÜGEL, S. ; JAMET, M.. Evidence for spin-to-charge conversion by Rashba coupling in metallic states at the Fe/Ge(111) interface. 7: 13857, Dec 2016. Article.

[108] HUBBARD, J.. Electron correlations in narrow energy bands. Proceedings of the Royal Society of London A: Mathematical, Physical and Engineering Sciences, 276 (1365): 238-257, 1963.

[109] GÓMEZ-SILVA, G.; ORELLANA, P. A. ; ANDA, E. V.. Enhancement of the thermoelectric efficiency in a T-shaped quantum dot system in the linear and nonlinear regimes. Journal of Applied Physics, 123 (8): 085706, 2018.

[110] DATTA, S.. Electronic Transport in Mesoscopic Systems. Cambridge Studies in Semiconductor Physics and Microelectronic Engineering. Cambridge University Press, 1995. 
[111] ANDA, E. V.; FLORES, F.. The role of inelastic scattering in resonant tunnelling heterostructures. Journal of Physics: Condensed Matter, 3 (46): 9087, 1991.

[112] BERTONI, G.; CALMELS, L.; ALTIBELLI, A. ; SERIN, V.. Firstprinciples calculation of the electronic structure and EELS spectra at the graphene/Ni(111) interface. Phys. Rev. B, 71: 075402, Feb 2005.

[113] ODASHIMA, M. M.; PRADO, B. G. ; VERNEK, E.. Pedagogical introduction to equilibrium Green's functions: Condensed-matter examples with numerical implementations. Revista Brasileira de Ensino de Física, 39, 002017.

[114] ECONOMOU, E. N.. Green's Functions in Quantum Physics. Springer Series in Solid-State Sciences 7. Springer, Berlin, Heidelberg, 3 edition, 2006.

[115] ZORNBERG, E. I.. Band structure and Fermi surface of ferromagnetic nickel. Phys. Rev. B, 1: 244-263, Jan 1970.

[116] GEORGES, A.; KOTLIAR, G.; KRAUTH, W. ; ROZENBERG, M. J.. Dynamical mean-field theory of strongly correlated fermion systems and the limit of infinite dimensions. Rev. Mod. Phys., 68: 13-125, Jan 1996.

[117] HOFSTETTER, W.; KEHREIN, S.. Symmetric Anderson impurity model with a narrow band. Phys. Rev. B, 59: R12732-R12735, May 1999.

[118] DIAS DA SILVA, L. G. G. V.; SANDLER, N. P.; INGERSENT, K. ; ULLOA, S. E.. Zero-field Kondo splitting and quantum-critical transition in double quantum dots. Phys. Rev. Lett., 97: 096603, Aug 2006.

[119] ZHURAVLEV, A. K.; IRKHIN, V. Y.. Kondo effect in the presence of van Hove singularities: A numerical renormalization group study. Phys. Rev. B, 84: 245111, Dec 2011.

[120] MASTROGIUSEPPE, D.; WONG, A.; INGERSENT, K.; ULLOA, S. E. ; SANDLER, N.. Quantum phase transitions into Kondo states in bilayer graphene. Phys. Rev. B, 89: 081101, Feb 2014.

[121] FANG, T.-F.; TONG, N.-H.; CAO, Z.; SUN, Q.-F. ; LUO, H.-G.. Spin susceptibility of Anderson impurities in arbitrary conduction bands. Phys. Rev. B, 92: 155129, Oct 2015. 
[122] LANGRETH, D. C.. Friedel sum rule for Anderson's model of localized impurity states. Phys. Rev., 150: 516-518, Oct 1966. 
A

\section{Formalismo de Operadores de Projeção}

\section{A.1 \\ Descrição do método}

Apresentamos neste apêndice uma discussão sucinta da abordagem do formalismo de operadores de projeção (POA), acrônimo originário do inglês projection operator approach, adequada para o estudo de sistemas fortemente correlacionados. É um método algébrico que permite calcular a energia do estado fundamental do sistema em função de seus parâmetros [24-26]. O formalismo é não perturbativo e seus resultados concordam para o caso do Hamiltoniano de Anderson para uma impureza com os obtidos por outros métodos [24]. O método foi desenvolvido para o Hamiltoniano de uma impureza de Anderson [7, 57]. Por este formalismo, é possível obter a magnetização e susceptibilidade magnética investigando a resposta do sistema a um campo magnético externo, da qual é possível extrair a temperatura Kondo e verificar excelente concordância com a lei de escala bem conhecida na literatura [24, 25].

O formalismo projeta o espaço de Hilbert no qual o Hamiltoniano está representado em um subespaço mais simples. A fim de operar neste subespaço, o Hamiltoniano deve ser renormalizado autoconsistentemente permitindo, assim, obter as propriedades do estado fundamental do sistema. A partir da energia do estado fundamental, $E$, é possível determinar, de forma praticamente exata, as propriedades estáticas do sistema, e.g. ocupação eletrônica na impureza, a magnetização, a susceptibilidade magnética e, usando as propriedades de um líquido de Fermi [7], a temperatura Kondo [26, 24, 25]. Além disso, com a regra da soma de Friedel [7, 122] é possível obter a condutância do sistema.

Com a finalidade de obter a energia do estado fundamental $E$ do sistema, o espaço de Hilbert é decomposto em dois subespaços, denotados por $S_{1}$ e $S_{2}$, obtidos aplicando os operadores de projeção $P_{1}$ e $P_{2}$ sobre o espaço de Hilbert onde o Hamiltoniano é representado. Definimos os estados pertencentes aos subespaço $S_{i}$, onde $i=1$ ou 2 , como $|1\rangle$ e $|2\rangle$. Logo, escrevemos os operadores como

$$
P_{i}=|i\rangle\langle i|
$$

e, desde que todo o espaço de Hilbert esteja contido nestes dois subespaços, temos por completeza que 


$$
\sum_{i} P_{i}=1
$$

Ainda, todo operador definido no espaço de Hilbert pode ser representado por uma combinação linear de $|1\rangle$ e $|2\rangle$, como veremos a seguir.

Aplicando $\sum_{i} P_{i}$ em ambos os lados do Hamiltoniano $H$, obtemos

$$
H=\sum_{i, j} H_{i j}
$$

onde $H_{i j} \equiv P_{i} H P_{j}=|i\rangle\langle i|H| j\rangle\langle j|$.

Um autoestado $\psi$ da equação de Schrödinger,

$$
H|\psi\rangle=E|\psi\rangle
$$

pode ser reescrito como uma soma de $\psi_{1}$ e $\psi_{2}$, onde $\psi_{i}$ é a componente do subespaço $i$. Com o auxílio da equação (A-3), a equação de Schrödinger em notação matricial fica

$$
\left(\begin{array}{ll}
H_{11} & H_{12} \\
H_{21} & H_{22}
\end{array}\right)\left(\begin{array}{l}
\psi_{1} \\
\psi_{2}
\end{array}\right)=E\left(\begin{array}{l}
\psi_{1} \\
\psi_{2}
\end{array}\right)
$$

É possível representar esta equação como um sistema de duas equações,

$$
H_{11} \psi_{1}+H_{12} \psi_{2}=E \psi_{1}
$$

e

$$
H_{21} \psi_{1}+H_{22} \psi_{2}=E \psi_{2},
$$

cuja solução no subespaço $S_{1}$ é

$$
H_{11} \psi_{1}+H_{12}\left(E-H_{22}\right)^{-1} H_{21} \psi_{1}=E \psi_{1}
$$

A equação de Schrödinger foi mapeada, desta forma, em uma nova equação com os autovalores originais preservados, atuando somente sobre a autofunção $\psi_{1}$ no subespaço projetado $S_{1}$.

Entretanto, encontrar a autoenergia $E$ do sistema, o estado fundamental, por exemplo, vai requerer a solução autoconsistente da equação (A-8) que pode ser escrita como

$$
H_{r} \psi_{1}=E \psi_{1},
$$

onde $H_{r}$ é o Hamiltoniano renormalizado dado por:

$$
H_{r} \equiv H_{11}+H_{12}\left(E-H_{22}\right)^{-1} H_{21} \text {. }
$$




\section{A. 2}

\section{Aplicação do formalismo de operadores de projeção ao Hamiltoniano de Anderson}

$\mathrm{Na}$ seção anterior, descrevemos a estrutura formal dos operadores de projeção, ao passo que nesta faremos uma abordagem mais pragmática, visando resolver o Hamiltoniano de Anderson, equação (A-11),

$$
H=\sum_{\sigma} \epsilon_{0 \sigma} n_{0 \sigma}+U n_{0 \sigma} n_{0 \bar{\sigma}}+\sum_{\boldsymbol{k}, \sigma} \epsilon_{\boldsymbol{k} \sigma} n_{\boldsymbol{k} \sigma}+\sum_{\boldsymbol{k}, \sigma} V_{\boldsymbol{k}}\left(d_{0 \sigma} c_{\boldsymbol{k} \sigma}^{\dagger}+d_{0 \sigma}^{\dagger} c_{\boldsymbol{k} \sigma}\right) .
$$

A abordagem dos operadores de projeção requer uma escolha conveniente do subespaço $S_{1}$. Adotaremos o estado com o mar de Fermi completamente preenchido até o nível de Fermi $\epsilon_{F}$ e a impureza de Anderson vazia. Este subespaço apresenta apenas um único estado a função, $\psi_{1}$. Neste caso a equação (A-9) pode ser reescrita como

$$
\left\langle 1\left|H_{r}(E)\right| 1\right\rangle=E
$$

que deve ser resolvida autoconsistentemente para obter a energia.

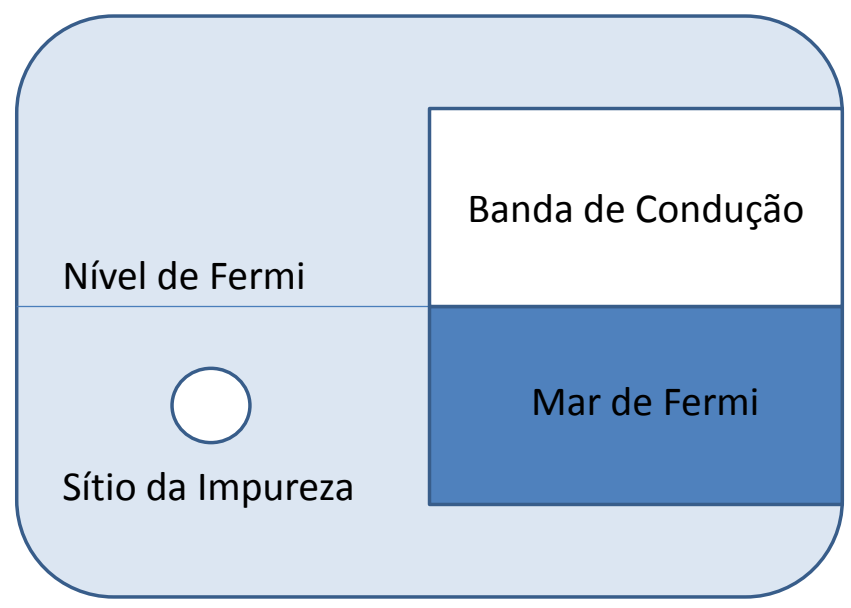

Figura A.1: Representação diagramática do subespaço $S_{1}$, composto por um único estado com o do mar de Fermi cheio e a impureza e a banda de condução vazias.

No cálculo da equação (A-12) obtemos um primeiro termo,

$$
h_{11} \equiv\left\langle 1\left|H_{11}\right| 1\right\rangle=\epsilon_{T}=\sum_{\epsilon_{\boldsymbol{k} \sigma}<\epsilon_{F}} \epsilon_{\boldsymbol{k} \sigma},
$$


que representa a contribuição à energia de todos os elétrons de condução $\epsilon_{T}$, que ocupam o mar de Fermi até o nível de Fermi $\epsilon_{F}$.

A análise do segundo termo deve ser mais cuidadosa. Primeiro devemos encontrar qual será o subespaço $S_{2}$ para então compreender o papel de cada operador neste segundo termo. Se por um lado o subespaço $S_{1}$ é muito simples, com apenas um estado, por outro, o subespaço $S_{2}$ é mais complexo, pois é composto por um conjunto infinito de estado não pertencentes ao subespaço $S_{1}$, ou expressando este conceito matematicamente a partir da equação (A-2), $P_{2}=1-P_{1}$.

A conexão entre os subespaços conforme aqui definidos é identificada como o termo de hibridização do Hamiltoniano de Anderson, cuja aplicação permite a passagem do subespaço $S_{1}$ para o $S_{2}$ através do operador $H_{21}$. Ele aniquila um elétron no mar de Fermi e cria um elétron na impureza,

$$
H_{21}=\sum_{k, \sigma} V_{\boldsymbol{k}}\left(d_{0 \sigma}^{\dagger} c_{\boldsymbol{k} \sigma}\right)
$$

O caminho de volta, do subespaço $S_{2}$ para o $S_{1}$, é dado pelo seu hermitiano conjugado,

$$
H_{12}=\sum_{\boldsymbol{k}, \sigma} V_{\boldsymbol{k}}\left(d_{0 \sigma} c_{\boldsymbol{k} \sigma}^{\dagger}\right)
$$

onde $c_{\boldsymbol{k} \sigma}^{\dagger}\left(c_{\boldsymbol{k} \sigma}\right)$ é o operador de criação (aniquilação) de um elétron no mar de Fermi com momento $\boldsymbol{k}$ e spin $\sigma$ e $d_{0 \sigma}^{\dagger}\left(d_{0 \sigma}\right)$ o de criação (aniquilação) de um elétron na impureza.

Ainda resta definir o operador $H_{22}$, que não possui uma expressão simples como as dos operadores $H_{11}, H_{21}$ e $H_{12}$ devido à complexidade do sistema. Todavia, isto não será um entrave para o cálculo da energia que será discutido a partir de agora.

Conforme já mencionado, partiremos do estado definido pela autofunção $\psi_{1}$ do subespaço $S_{1}$, com $N$ elétrons no estado fundamental do mar de Fermi. A atuação do operador $H_{21}$ sobre $\psi_{1}$ cria um elétron localizado na impureza e um buraco no mar de Fermi, o qual chamaremos de estados I. É importante enfatizar que não é apenas um estado, mas uma família de estados análogos, em virtude do buraco no mar de Fermi poder possuir infinitos valores possíveis do momento $\boldsymbol{k}$. Logo, a família de estados I é composta sempre pela impureza ocupada por um elétron e um buraco no mar de Fermi com momento $k$, uma família de infinitos estados no limite termodinâmico.

Não obstante, cada aplicação sucessiva dos operadores $H_{12}$ e $H_{21}$ geram uma nova família de estados que chamaremos de estados I, estados II e assim por diante, todos pertencentes ao subespaço $S_{2}$ e genericamente representados pela autofunção $\psi_{2}$, onde o algarismo romano representa um nível hierárquico 
estabelecido pelo número de aplicações dos operadores $H_{12}$ e $H_{21}$.

Antes de continuarmos rumo a família de estados II, salientamos que quase na totalidade desta tese estaremos tratando os sistemas com a interação Coulombiana na impureza $U$ infinita. No que diz respeito ao formalismo dos operadores de projeção, sempre utilizaremos este limite, pois esta aproximação simplifica muito o tratamento numérico deste método conforme ficará mais evidente adiante. Sistemas descritos por esta restrição são tipicamente terras raras ou metais de transição, uma vez que a localização das funções de onda eletrônicas nas camadas $3 \mathrm{~d}$ ou $4 \mathrm{f}$ originam uma forte repulsão Coulombiana, segundo mencionado na seção 1.4 do capítulo 1. Portanto, a dupla ocupação na impureza não estará no espaço de Hilbert que trabalharemos, apesar deste poder ser incluído usando o formalismo de operadores de projeção [25]. Com a finalidade de tratar sistemas com repulsão Coulombiana finita, que faremos mais adiante, utilizaremos outros formalismos.

Como a dupla ocupação na impureza não está permitida, dos estados I podemos apenas ir a estados II em que a impureza está vazia, levando o elétron da impureza à banda de condução, o qual é possível com o operador $H_{12}$ destruindo o elétron na impureza e criando-o na banda de condução acima do nível de Fermi. Denotaremos o momento dos elétrons cujo elemento diagonal está por cima do nível de Fermi por letras maiúsculas, $\boldsymbol{K}$, ou seja, os estados da banda de condução são escritos como $\epsilon_{\boldsymbol{K}}$, para todo $\epsilon_{\boldsymbol{K}}>\epsilon_{F}$. Entretanto, o elétron localizado na impureza poderia ocupar o buraco existente no mar de Fermi com a aplicação do operador $H_{12}$, resultando em um estado II diferente do descrito. Contudo, para isto ocorrer o elétron deveria possuir um momento $\boldsymbol{k}$ específico igual ao do buraco, frente a todos os valores possíveis de $\boldsymbol{k}$ da banda de condução, os quais constituem um somatório. Conforme ficará evidente no decorrer do texto, o elétron da impureza ocupar o buraco no mar de Fermi não envolve um somatório em $\boldsymbol{k}$, pois está dirigindo-se a um estado específico e não a um arbitrário como no caso da banda de condução, resultando em não fornecer contribuições ao cálculo no limite termodinâmico.

Nos estados II, com a impureza vazia, podemos novamente aplicar o operador $H_{12}$ e continuar este processo indefinidamente, conforme a ilustração na figura A.2, visto que no limite termodinâmico o mar de Fermi tende a um contínuo.

Segundo o diagrama da figura A.2 e pelo discutido até o momento, observa-se que os estados III são análogos aos estados I, a menos de um buraco no mar de Fermi e de um elétron na banda de condução. Ambas as famílias de estados surgem da criação de um elétron na impureza e aniquilação de um elétron no mar de Fermi. É este fato que possibilita, como veremos, a solução 


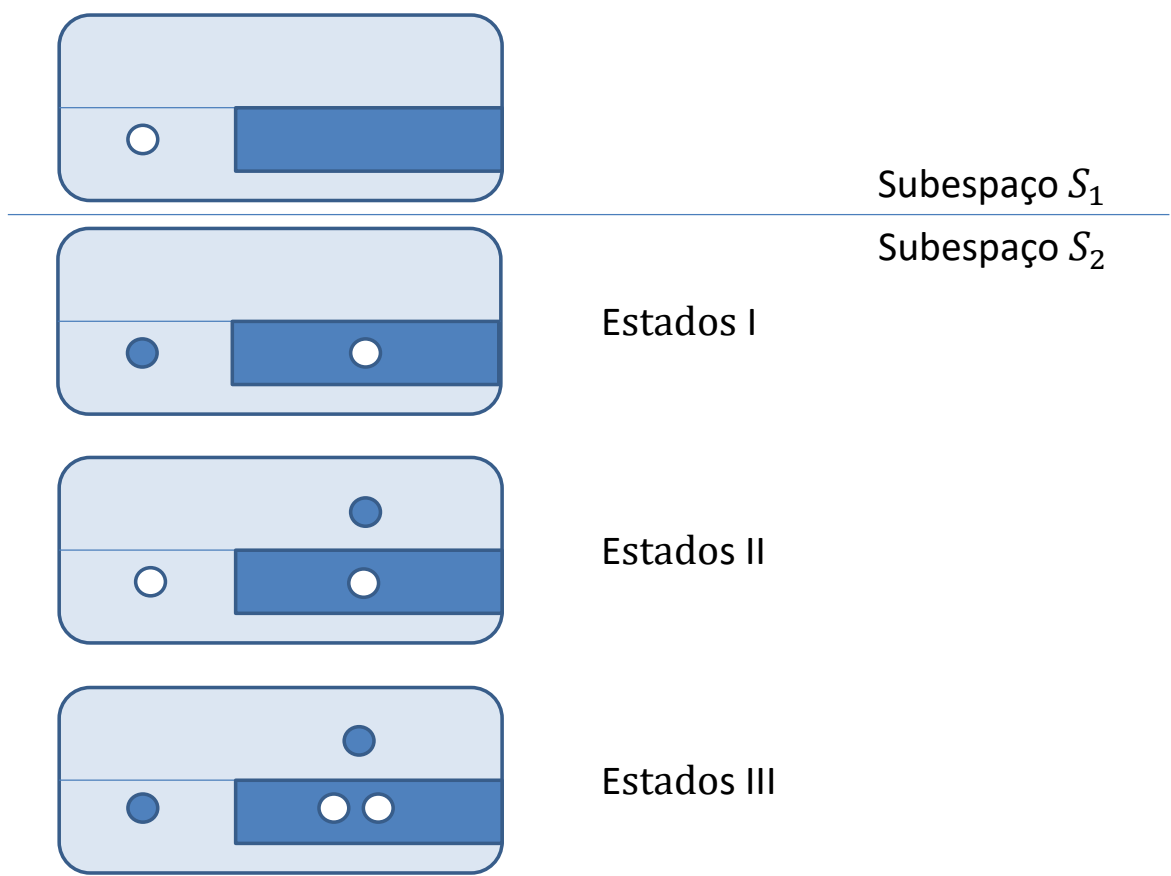

Figura A.2: Construção do subespaço $S_{2}$, representando esquematicamente três famílias de estados construídas com sucessivas aplicações do operador $H_{21}$.

autoconsistente do problema.

Suporemos que a hibridização independa de $\boldsymbol{k}$ e seja uma constante igual a $V$, como é suposto habitualmente. Além disso, ela escala com $1 / \sqrt{N}$, sendo $N$ o número de estados no mar de Fermi, a fim de preservar o valor finito das energias,

$$
V_{k}=\frac{V}{\sqrt{N}} \quad \forall \quad k .
$$

O cálculo da energia a partir do Hamiltoniano renormalizado, equação (A-10), origina dois termos, dos quais já conhecemos o primeiro expresso na equação (A-13), restando calcular o segundo. Como a forma dos operadores $H_{12}$ e $H_{21}$ já são conhecidas, resta apenas inverter o operador $\left(E-H_{22}\right)$, o qual está situado no subespaço $S_{2}$, e encontrar o seu elemento de matriz correspondente imediatamente contíguo ao subespaço $S_{1}$.

Definimos a correção à energia do estado fundamental como:

$$
\Delta E=E-\epsilon_{T}
$$

O cálculo da energia com a renormalização que surge de inverter o operador $\left(E-H_{22}\right)$ é iniciado levando-se em consideração os estados I, que se originam da aplicação do $H_{21}$ sobre $\psi_{1}$, resultando em

$$
\Delta E=\frac{V^{2}}{E-h_{22}^{(I)}} \equiv \Sigma_{I},
$$


onde definimos a contribuição da família de estados I como $\Sigma_{I}$ e $h_{22}^{(I)}$ como o elemento de matriz do operador $H_{22}$ considerando apenas a correção oriunda dos estados I. O numerador de $\Sigma_{I}$ é oriundo dos operadores $H_{21}$ e $H_{12}$, equações (A-14) e (A-15), respectivamente.

Substituímos $h_{22}^{(I)}$ pelas correções provenientes da família de estados I e estados II. Sendo que as correções oriundas dos estados II, através da quantidade $\Sigma_{I I}$, ainda devem ser calculadas. Então, a equação (A-18) fica

$$
\Delta E=\frac{1}{N} \sum_{\epsilon_{\boldsymbol{k} \sigma}<\epsilon_{F}} \frac{V^{2}}{\Delta E+\epsilon_{\boldsymbol{k} \sigma}-\epsilon_{0 \sigma}-\Sigma_{I I}} .
$$

O somatório para $\epsilon_{\boldsymbol{k} \sigma}<\epsilon_{F}$ provém de incorporar as contribuições de todos os elétrons por baixo do nível de Fermi, os quais constituem a família de estados I.

Observe que a fim de calcular a energia dos estados I precisamos conhecer a energia dos estados II, $\Sigma_{I I}$, como comentamos. A correção associada aos estados II pode ser escrita como

$$
\Sigma_{I I}=\frac{V^{2}}{E-h_{22}^{(I I)}}=\sum_{\epsilon_{\boldsymbol{K} \sigma}>\epsilon_{F}} \frac{V^{2}}{\Delta E+\epsilon_{\boldsymbol{k} \sigma}-\epsilon_{\boldsymbol{K} \sigma}-\Sigma_{I I I}},
$$

que naturalmente depende dos estados III, a partir de $\Sigma_{I I I}$.

Seguindo com o processo, escrevemos

$$
\Sigma_{I I I}=\frac{V^{2}}{E-h_{22}^{(I I I)}}=\sum_{\epsilon_{\boldsymbol{k}^{\prime} \sigma}<\epsilon_{F}} \frac{V^{2}}{\Delta E+\epsilon_{\boldsymbol{k} \sigma}-\epsilon_{\boldsymbol{K} \sigma}+\epsilon_{\boldsymbol{k}^{\prime} \sigma}-\epsilon_{0 \sigma}-\Sigma_{I V}},
$$

com o somatório em $\epsilon_{\boldsymbol{k}^{\prime} \sigma}<\epsilon_{F}$ devido ao segundo elétron destruído no mar de Fermi, que deve satisfazer $\boldsymbol{k}^{\prime} \neq \boldsymbol{k}$, pois o elétron $\boldsymbol{k}$ já foi aniquilado no estado anterior.

Os estados III também devem ser corrigidos pelas novas famílias de estados atingidas, de níveis hierárquicos superiores e representadas nesta última equação por $\Sigma_{I V}$. Entretanto, observe a já esperada similaridade da renormalização da energia, devido aos estados III serem análogos aos estados I, resultando ser possível identificar uma simetria de recorrência.

Com a finalidade de ressaltar a estrutura de fração continuada que surge deste processo de criação de famílias de estados, reescrevemos a equação (A-19) incorporando as correções descritas há pouco.

$$
\begin{array}{r}
\Delta E=\frac{1}{N} \sum_{\epsilon_{\boldsymbol{k} \sigma}<\epsilon_{F}} \frac{V^{2}}{\Delta E+\epsilon_{\boldsymbol{k} \sigma}-\epsilon_{0 \sigma}-\frac{1}{N} \sum_{\epsilon_{\boldsymbol{K} \sigma}>\epsilon_{F}} \frac{V^{2}}{\Delta E+\epsilon_{\boldsymbol{k} \sigma}-\epsilon_{\boldsymbol{K} \sigma}-\Sigma}} \\
\Sigma=\frac{1}{N} \sum_{\epsilon_{\boldsymbol{k}^{\prime} \sigma}<\epsilon_{F}} \frac{V^{2}}{\Delta E+\epsilon_{\boldsymbol{k} \sigma}-\epsilon_{\boldsymbol{K} \sigma}+\epsilon_{\boldsymbol{k}^{\prime} \sigma}-\epsilon_{0 \sigma}-\ldots}
\end{array}
$$


No limite termodinâmico, quando $N$ tende a infinito, e levando em consideração as renormalizações de estados de famílias de ordem superior, a equação (A-22) assume uma estrutura de fração continuada, permitindo um cálculo autoconsistente.

Definindo as funções

$$
f_{1 \sigma}(\xi) \equiv \frac{1}{N} \sum_{\epsilon_{\boldsymbol{k} \sigma}<\epsilon_{F}} \frac{V^{2}}{\xi+\epsilon_{\boldsymbol{k} \sigma}-\epsilon_{0 \sigma}-f_{0 \sigma}\left(\xi+\epsilon_{\boldsymbol{k} \sigma}\right)}
$$

$\mathrm{e}$

$$
f_{0 \sigma}(\xi) \equiv \frac{1}{N} \sum_{\epsilon_{\boldsymbol{K} \sigma}>\epsilon_{F}} \frac{V^{2}}{\xi-\epsilon_{\boldsymbol{K} \sigma}-\sum_{\sigma} f_{1 \sigma}\left(\xi-\epsilon_{\boldsymbol{K} \sigma}\right)},
$$

a equação (A-22) pode ser escrita de uma forma sintética, ficando evidente o cálculo autoconsistente e considerando os infinitos estágios de renormalizações como

$$
\Delta E=\sum_{\sigma} f_{1 \sigma}(\Delta E)
$$

Dada a relação $E=\Delta E+\epsilon_{T}$, a determinação da função $f_{1 \sigma}(\xi)$ permite, em princípio, obter a energia do estado fundamental do sistema.

No limite termodinâmico em que $N \rightarrow \infty$, eliminamos a restrição de $\boldsymbol{k} \neq \boldsymbol{k}^{\prime}$ e os somatórios em $\epsilon_{\boldsymbol{k} \sigma}$ são reescritos como

$$
\frac{1}{N} \sum_{\epsilon_{\boldsymbol{k} \sigma}} f\left(\epsilon_{\boldsymbol{k} \sigma}\right)=\int \frac{1}{N} \sum_{\epsilon_{\boldsymbol{k} \sigma}} \delta\left(x-\epsilon_{\boldsymbol{k} \sigma}\right) f(x) d x=\int \rho_{\sigma}(x) f(x) d x
$$

onde $\delta\left(x-x^{\prime}\right)$ é a função delta de Dirac e $\rho_{\sigma}(x)$ a densidade de estados, definida como

$$
\rho_{\sigma}(x) \equiv \frac{1}{N} \sum_{\epsilon_{\boldsymbol{k} \sigma}} \delta\left(x-\epsilon_{\boldsymbol{k} \sigma}\right) .
$$

Neste limite, as funções nas equações (A-23) e (A-24) são escrita como

$$
f_{1 \sigma}(\xi) \equiv V^{2} \int_{-\infty}^{\epsilon_{F}} \frac{\rho_{\sigma}(x)}{\xi+x-\epsilon_{0 \sigma}-f_{0 \sigma}(\xi+x)} d x
$$

e

$$
f_{0 \sigma}(\xi) \equiv V^{2} \int_{\epsilon_{F}}^{\infty} \frac{\rho_{\sigma}(x)}{\xi-x-\sum_{\sigma} f_{1 \sigma}(\xi-x)} d x .
$$

As renormalizações devidas aos estados I e III na equação (A-22) tornam-se iguais, de modo que o problema consiste em resolver autoconsistentemente as equações (A-28) e (A-29) de onde é possível obter, pela equação (A-25), a energia $E[24,26]$.

\section{A.3}

\section{Equivalência do formalismo usando funções de Green}

Uma forma alternativa de obter a correção da energia ao estado fundamental consiste em utilizar a equivalência deste problema com o formalismo das funções de Green [38]. Esta equivalência surge do fato de que o operador $\left(E-H_{22}\right)^{-1}$ é o operador resolvente cujo elemento de matriz deve ser calcu- 
lado nos estados que foram definidos como I no subespaço $S_{2}$. Esta estrutura é uma função de Green cuja equação de movimento define uma rede de Bethe como mostramos na figura A.3

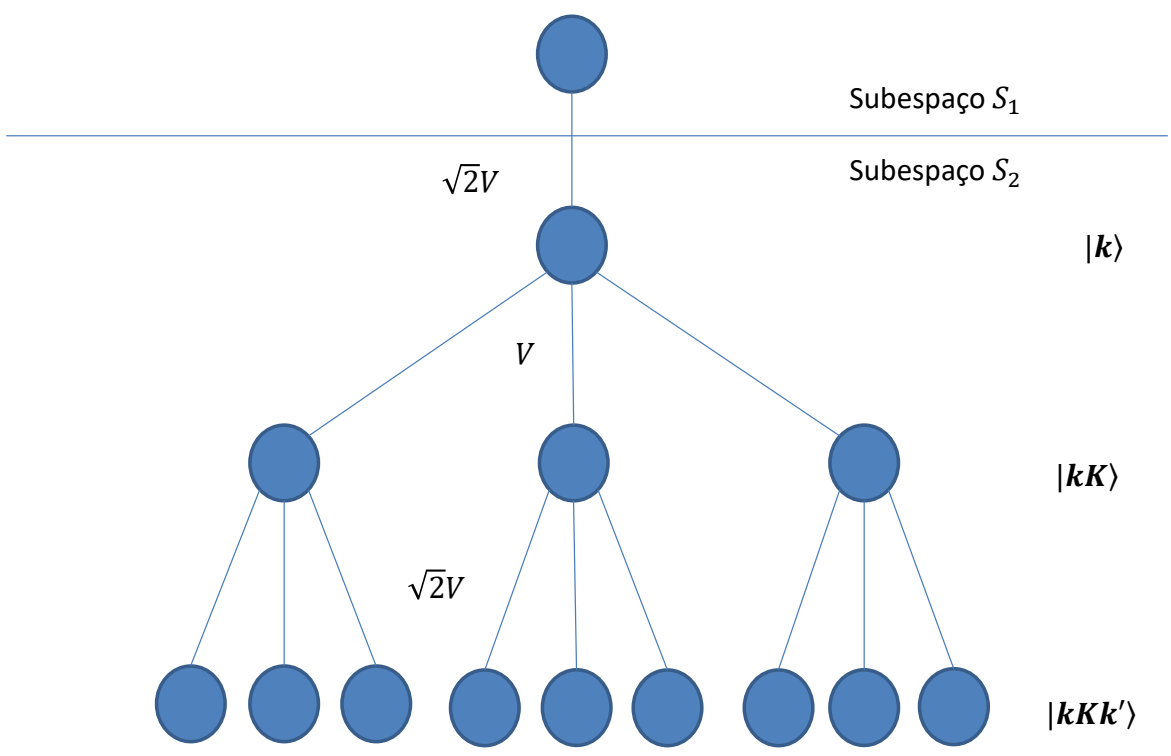

Figura A.3: Analogia dos sítios de uma rede de Bethe com os estados construídos com o formalismo de operadores de projeção.

Associando uma função de Green a cada sítio da rede de Bethe podemos escrever as equações

$\mathrm{e}$

$$
\begin{gathered}
\left\langle\boldsymbol{k}\left|\left(E-H_{22}\right)^{-1}\right| \boldsymbol{k}\right\rangle=G_{\boldsymbol{k}, \boldsymbol{k}}=g_{\boldsymbol{k}}+g_{\boldsymbol{k}} \frac{V}{\sqrt{N}} \sum_{K} G_{\boldsymbol{k} K, \boldsymbol{k}}, \\
G_{\boldsymbol{k} K, \boldsymbol{k}}=g_{\boldsymbol{k} \boldsymbol{K}} \frac{\sqrt{2} V}{\sqrt{N}} \sum_{\boldsymbol{k}^{\prime}} G_{\boldsymbol{k} \boldsymbol{K} \boldsymbol{k}^{\prime}, \boldsymbol{k}}+g_{\boldsymbol{k} K} \frac{V}{\sqrt{N}} G_{\boldsymbol{k}, \boldsymbol{k}}
\end{gathered}
$$

$$
G_{k K k^{\prime}, \boldsymbol{k}}=g_{\boldsymbol{k} K \boldsymbol{k}^{\prime}} \frac{\sqrt{2} V}{\sqrt{N}} G_{\boldsymbol{k} K \boldsymbol{k}, \boldsymbol{k}}
$$

onde $g_{k}$ é a função de Green de um corpo em um único sítio da família de estados I, a qual é caracterizada pelo momento $k$ do buraco criado no mar de Fermi, e $G_{k, k}$ é a função de Green vestida com a interação que conecta os sítios da rede, que consiste no termo de hibridização do Hamiltoniano de Anderson, elemento de matriz não diagonal, de magnitude $V$. De modo similar são definidas as funções de Green de um corpo $g_{\boldsymbol{k} K}$ e $g_{\boldsymbol{k} K \boldsymbol{k}^{\prime}}$, onde $g_{\boldsymbol{k} \boldsymbol{K}}$ se refere aos estados com um buraco no mar de Fermi e um elétron na banda de condução e $g_{\boldsymbol{k} \boldsymbol{K} \boldsymbol{k}^{\prime}}$ aos estados com dois buracos no mar de Fermi, um elétron na banda de condução e um elétron ocupando a impureza. Os somatório em 
$K$ e $\boldsymbol{k}^{\prime}$ são originários de levar o elétron da impureza à banda de condução e a criação do segundo buraco no mar de Fermi, respectivamente. O fator $\sqrt{2}$ presente deve-se a antissimetrização devido ao spin, visto que o estado inicial do subespaço $S_{1}$ é um singleto e como o spin é um bom número quântico no Hamiltoniano de Anderson, devemos formar combinações lineares de estados com spin total nulo.

Resolvendo este sistema de equações, equações (A-30), (A-31) e (A-32), temos a função de Green no sítio adjunto do subespaço $S_{1}$,

$$
G_{k, k}=\frac{g_{k}}{1-g_{k} \frac{V^{2}}{N} \sum_{K} \frac{g_{k K}}{1-g_{k K} \frac{2 V^{2}}{N} \sum_{k^{\prime}} g_{k K k^{\prime}}}} .
$$

Para efetuar a passagem do sítio contíguo ao subespaço $S_{1}$ ao próprio subespaço $S_{1}$, é efetuada a soma sobre $k$ e a multiplicação por $(\sqrt{2} V / \sqrt{N})^{2}$, onde, novamente, o fator $\sqrt{2}$ é devido ao singleto e o expoente quadrado pois saímos do subespaço $S_{1}$, fomos a todos as famílias de estados do subespaço $S_{2}$ e voltamos ao subespaço $S_{1}$, obtendo

$$
\frac{2 V^{2}}{N} \sum_{\boldsymbol{k}} G_{\boldsymbol{k}, \boldsymbol{k}}=\frac{2 V^{2}}{N} \sum_{\boldsymbol{k}} \frac{g_{\boldsymbol{k}}}{1-g_{\boldsymbol{k}} \frac{V^{2}}{N} \sum_{\boldsymbol{K}} \frac{g_{\boldsymbol{k} \boldsymbol{K}}}{1-g_{\boldsymbol{k} \boldsymbol{K}} \frac{2 V^{2}}{N} \sum_{\boldsymbol{k}^{\prime}} g_{\boldsymbol{k} \boldsymbol{K} \boldsymbol{k}^{\prime}}}} .
$$

Restando apenas apresentar as expressões para as funções de Green de um corpo,

$$
\begin{gathered}
g_{\boldsymbol{k}}=\frac{1}{\Delta E+\epsilon_{\boldsymbol{k}}-\epsilon_{0}}, \\
g_{\boldsymbol{k} \boldsymbol{K}}=\frac{1}{\Delta E+\epsilon_{\boldsymbol{k}}-\epsilon_{\boldsymbol{K}}} \\
g_{\boldsymbol{k} K \boldsymbol{k}^{\prime}}=\frac{1}{\Delta E+\epsilon_{\boldsymbol{k}}+\epsilon_{\boldsymbol{k}^{\prime}}-\epsilon_{\boldsymbol{K}}-\epsilon_{0}} .
\end{gathered}
$$

$\mathrm{e}$

Ressaltamos que no cálculo da equação (A-34) só fomos até o terceiro nível hierárquico da rede de Bethe, com $g_{\boldsymbol{k} \boldsymbol{K} \boldsymbol{k}^{\prime}}$, mas observa-se claramente a estrutura de fração continuada presente tanto nesta equação como na discutida na seção anterior. A fim de levar em consideração todas as famílias de estados que poderiam ser percorridas, definimos a função de Green de um corpo renormalizada por todos estes sítios como

$$
\tilde{g}_{\boldsymbol{k} K \boldsymbol{k}^{\prime}}=\frac{1}{\Delta E+\epsilon_{\boldsymbol{k}}+\epsilon_{\boldsymbol{k}}-\epsilon_{\boldsymbol{K}}-\epsilon_{0}-f_{0 \sigma}\left(\Delta E+\epsilon_{\boldsymbol{k}}+\epsilon_{\boldsymbol{k}^{\prime}}-\epsilon_{\boldsymbol{K}}-\epsilon_{0}\right)},
$$

com as devidas definições de $f_{1 \sigma}(\xi)$ e $f_{0 \sigma}(\xi)$, equações (A-23) e (A-24). Assim, substituindo $g_{\boldsymbol{k} K \boldsymbol{k}^{\prime}}$ por $\tilde{g}_{\boldsymbol{k} K \boldsymbol{k}^{\prime}}$ na equação (A-34) e usando as funções de Green despidas, equações (A-35) a (A-37), obtemos a mesma expressão para a correção da energia do estado fundamental, que consiste na equação (A-25).

Esta proposta de cálculo usando as funções de Green é mais clara que a anterior e também mais acessível em um primeiro contato com o formalismo, 
além disso, permite obter outras contribuições à energia total do estado fundamental do sistema, que ficará evidente na próxima seção.

\section{A.4}

\section{Importância dos termos não diagonais}

Quando calculamos a função de Green $G_{k, k}$ na seção anterior, partimos de um estado com momento $k$ bem definido e percorremos todos os outros estados que surgem a partir deste, originando $K$ e $\boldsymbol{k}^{\prime}$, e voltamos ao mesmo $\boldsymbol{k}$ original. Ou seja, o termo calculado $G_{k, k}$ consiste em um elemento diagonal. Porém, poderíamos ter retornado a um valor de momento $\boldsymbol{k}^{\prime}$, caracterizando um termo não diagonal $G_{\boldsymbol{k}, \boldsymbol{k}^{\prime}}$. Portanto, ao sair de um estado com um buraco no mar de Fermi, estado caracterizado por um momento $\boldsymbol{k}$, e retornarmos ao estado equivalente com também um buraco, mas rotulado por um momento $\boldsymbol{k}^{\prime}$, temos, necessariamente, que em um dado momento passar por um estado com dois buracos no mar de Fermi, que seriam $\boldsymbol{k}$ e $\boldsymbol{k}^{\prime}$. O menor "caminho" que pode ser feito com essas características é aquele que, começando com um buraco no mar de Fermi com momento $k$ e a impureza ocupada, seguir ao próximo estado levando o elétron da impureza à banda de condução, estado $\boldsymbol{k} \boldsymbol{K}$, criar um segundo buraco no mar de Fermi com momento $\boldsymbol{k}^{\prime}$, estado $\boldsymbol{k} \boldsymbol{K} \boldsymbol{k}^{\prime}$, retornar com o elétron da impureza para o mar de Fermi ocupando o momento $k$ de partida, estado $k^{\prime} K$, e, finalmente, trazer o elétron da banda de condução com momento $K$ para a impureza, estado $\boldsymbol{k}^{\prime}$, caminho representado no diagrama da figura A.4.

Escrevendo as funções de Green para o caminho proposto, temos,

$$
G_{k, k}=g_{k} \frac{V}{\sqrt{N}} \sum_{K} G_{k K, k}
$$

e

$$
\begin{gathered}
G_{k K, k}=\tilde{g}_{k K} \frac{V}{\sqrt{N}} G_{k, k}+\tilde{g}_{k K} \frac{\sqrt{2} V}{\sqrt{N}} G_{\boldsymbol{k} k K, \boldsymbol{k}}, \\
G_{\boldsymbol{k} \boldsymbol{k}, \boldsymbol{k}}=\tilde{g}_{\boldsymbol{k} \boldsymbol{k} K} \frac{\sqrt{2} V}{\sqrt{N}} G_{\boldsymbol{k} K, k}+\tilde{g}_{\boldsymbol{k} \boldsymbol{k} K} \frac{\sqrt{2} V}{\sqrt{N}} G_{\boldsymbol{k} K, \boldsymbol{k}}
\end{gathered}
$$

cuja solução é

$$
G_{k K, k}=\tilde{g}_{k K} \frac{\sqrt{2} V}{\sqrt{N}} G_{k k K, k}+\tilde{g}_{k K} \frac{V}{\sqrt{N}} G_{k, k}
$$

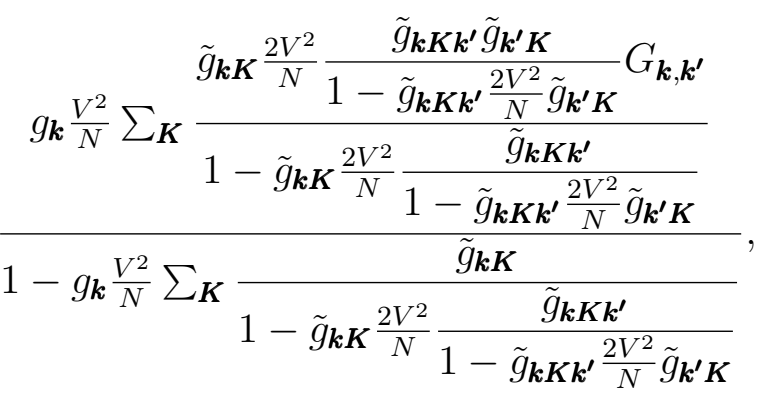




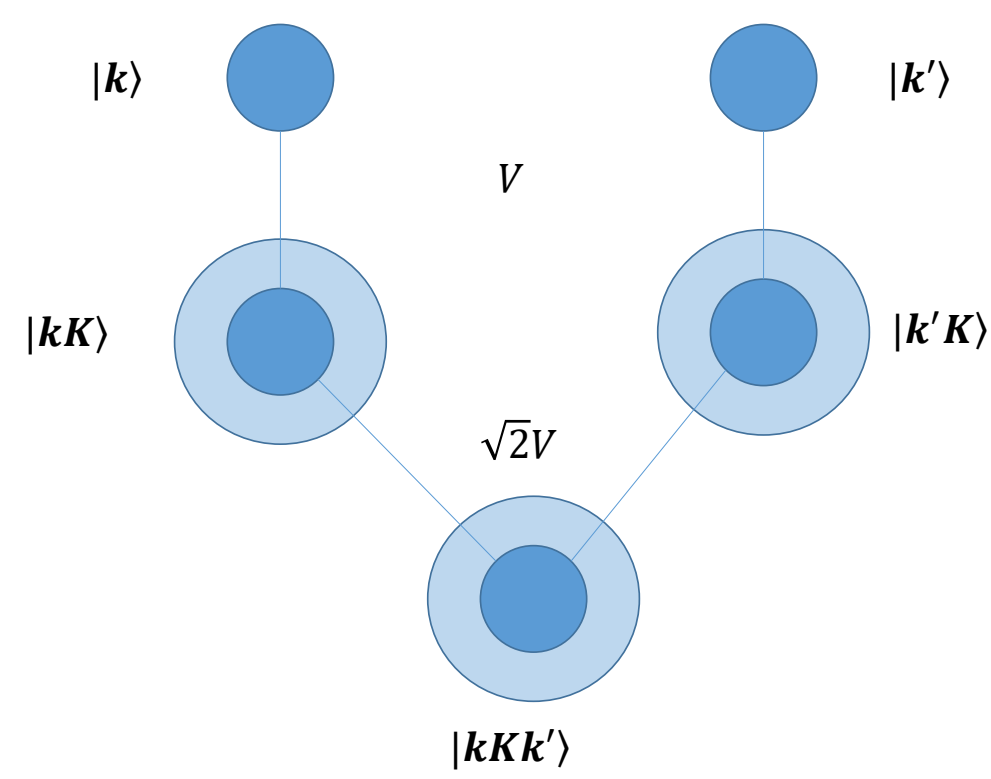

Figura A.4: Diagrama representando uma contribuição não diagonal. Os sítios destacados são renormalizados por termos diagonais.

onde as funções de Green despidas são renormalizadas por todos os estados possíveis que podem ser acessados pelo do termo diagonal.

As formas das funções de Green locais são

$$
\begin{gathered}
\tilde{g}_{\boldsymbol{k}}=\frac{1}{\Delta E+\epsilon_{\boldsymbol{k}}-\epsilon_{0}-f_{2 \sigma}\left(\Delta E+\epsilon_{k}-\epsilon_{0}\right)}, \\
\tilde{g}_{\boldsymbol{k} K}=\frac{1}{\Delta E+\epsilon_{\boldsymbol{k}}-\epsilon_{\boldsymbol{K}}-f_{1}\left(\Delta E+\epsilon_{\boldsymbol{k}}-\epsilon_{\boldsymbol{K}}\right)},
\end{gathered}
$$

$\mathrm{e}$

$$
\tilde{g}_{\boldsymbol{k} \boldsymbol{K} \boldsymbol{k}^{\prime}}=\frac{1}{\Delta E+\epsilon_{\boldsymbol{k}}+\epsilon_{\boldsymbol{k}^{\prime}}-\epsilon_{\boldsymbol{K}}-\epsilon_{0}-f_{2 \sigma}\left(\Delta E+\epsilon_{\boldsymbol{k}}+\epsilon_{\boldsymbol{k}^{\prime}}-\epsilon_{\boldsymbol{K}}-\epsilon_{0}\right)} .
$$

Diferentemente ao termo diagonal, o não diagonal envolve contribuições que não envolvem somatórios, logo elas são nulas, resultando em

$$
G_{\boldsymbol{k}, \boldsymbol{k}^{\prime}}=\frac{\frac{2 V^{4}}{N^{2}} g_{\boldsymbol{k}} \sum_{K} \tilde{g}_{\boldsymbol{k} \boldsymbol{K}} \tilde{g}_{\boldsymbol{k} \boldsymbol{K} \boldsymbol{k}^{\prime}} \tilde{g}_{\boldsymbol{k}^{\prime} K} G_{\boldsymbol{k}^{\prime}, \boldsymbol{k}^{\prime}}}{1-g_{\boldsymbol{k}} \frac{V^{2}}{N} \sum_{\boldsymbol{K}} \tilde{g}_{\boldsymbol{k} \boldsymbol{K}}}
$$

Somando para todo $\boldsymbol{k}$ e $\boldsymbol{k}^{\prime}$, multiplicando por $(\sqrt{2} V / \sqrt{N})^{2}$ e usando as definições das funções de Green despidas renormalizadas, obtemos a contribuição 
do termo não diagonal ao cálculo da energia do estado fundamental

$$
\begin{array}{r}
\frac{2 V^{2}}{N} \sum_{\boldsymbol{k}, \boldsymbol{k}^{\prime}} G_{\boldsymbol{k}, \boldsymbol{k}^{\prime}}=\frac{4 V^{6}}{N^{3}} \sum_{\boldsymbol{k}, \boldsymbol{K}, \boldsymbol{k}^{\prime}} \frac{1}{\Delta E+\epsilon_{\boldsymbol{k}}-\epsilon_{\boldsymbol{K}}-f_{1 \sigma}\left(\Delta E+\epsilon_{\boldsymbol{k}}-\epsilon_{\boldsymbol{K}}\right)} \times \\
\frac{1}{\Delta E+\epsilon_{\boldsymbol{k}}+\epsilon_{\boldsymbol{k}^{\prime}}-\epsilon_{\boldsymbol{K}}-\epsilon_{0}-f_{2 \sigma}\left(\Delta E+\epsilon_{\boldsymbol{k}}+\epsilon_{\boldsymbol{k}^{\prime}}-\epsilon_{\boldsymbol{K}}-\epsilon_{0}\right)} \times \\
\frac{1}{\Delta E+\epsilon_{\boldsymbol{k}^{\prime}}-\epsilon_{\boldsymbol{K}}-f_{1 \sigma}\left(\Delta E+\epsilon_{\boldsymbol{k}^{\prime}}-\epsilon_{\boldsymbol{K}}\right)} \times \\
\frac{1}{\Delta E+\epsilon_{\boldsymbol{k}}-\epsilon_{0}-f_{2 \sigma}\left(\Delta E+\epsilon_{\boldsymbol{k}}-\epsilon_{0}\right)} \times \\
\frac{1}{\Delta E+\epsilon_{\boldsymbol{k}^{\prime}}-\epsilon_{0}-f_{2 \sigma}\left(\Delta E+\epsilon_{\boldsymbol{k}^{\prime}}-\epsilon_{0}\right)}
\end{array}
$$

Tomando o limite termodinâmico, ou fazendo a passagem ao contínuo, vemos que os somatórios da equação (A-48) tornam-se uma integral múltipla, de difícil solução numérica. Para o caso mais simples de uma banda plana com semilargura de banda $D$, o valor desta contribuição não diagonal pode ser encontrado nas referências [24] para $U \rightarrow \infty$ e [25] para $U$ finito. Na situação $U \rightarrow \infty$, esta contribuição pode ser desprezada [24]. Uma forma de estimar a contribuição do termo não diagonal é verificar que se trata de um termo de pelo menos da ordem de $V^{4} / D^{3}$, como tipicamente $V \ll D$ a correção do termo não diagonal pode ser desprezada frente ao diagonal com excelentes resultados $[24,26]$. Contudo, quando $U$ é finito o termo não diagonal é da ordem de $V^{2} / D-$ também para uma banda plana - e sua importância começa a ser relevante [25]. Um cálculo rigoroso para esta argumentação pode ser encontrado nas referências [24, 25].

Ao longo desta tese estaremos abordando os problemas com o formalismo dos PO sempre no limite de $U \rightarrow \infty[24,26]$. Os casos com $U$ finito serão tratados com outros enfoques, apesar de ser possível utilizar o formalismo dos POA [25] para tal fim. Esta aproximação simplifica consideravelmente, do ponto de vista numérico e computacional, os cálculos, em virtude de não ter que não efetuar integrais múltiplas numericamente. Além disso, a escolha de $U \rightarrow \infty$ não muda a física dos sistemas em estudo.

\section{A.5}

\section{Extensão do formalismo de operadores de projeção para sistemas com duas impurezas}

Esta seção é centrada em discutir como estender o POA para sistemas descritos pelo Hamiltoniano de Anderson com duas impurezas, como o sistema de dois pontos quânticos (PQ) acoplados capacitivamente analisados no capítulo 2 . 
Conforme discutido ao longo deste apêndice, no formalismo de POA é importante escolher de forma adequada o subespaço $S_{1}$, onde o Hamiltoniano do modelo será projetado, resultando em um Hamiltoniano efetivo, com duas funções auxiliares que devem ser calculadas autoconsistentemente. No presente caso, com a finalidade tratar duas impurezas idênticas com interação Coulombiana intra-PQ infinita, generalizamos a escolha usual do subespaço $S_{1}$. Escolhemos este subespaço como composto por apenas um estado, representado pela função $\psi_{1}$, com os PQs descarregados e os dois mares de Fermi no estado fundamental, como ilustrado na figura A.5. Assim como no caso de apenas uma impureza, todos os outros estados pertencem ao subespaço $S_{2}$ serão acessados por uma aplicação sucessiva do Hamiltoniano $H_{21}$ ao único estado contido no subespaço $S_{1}$.

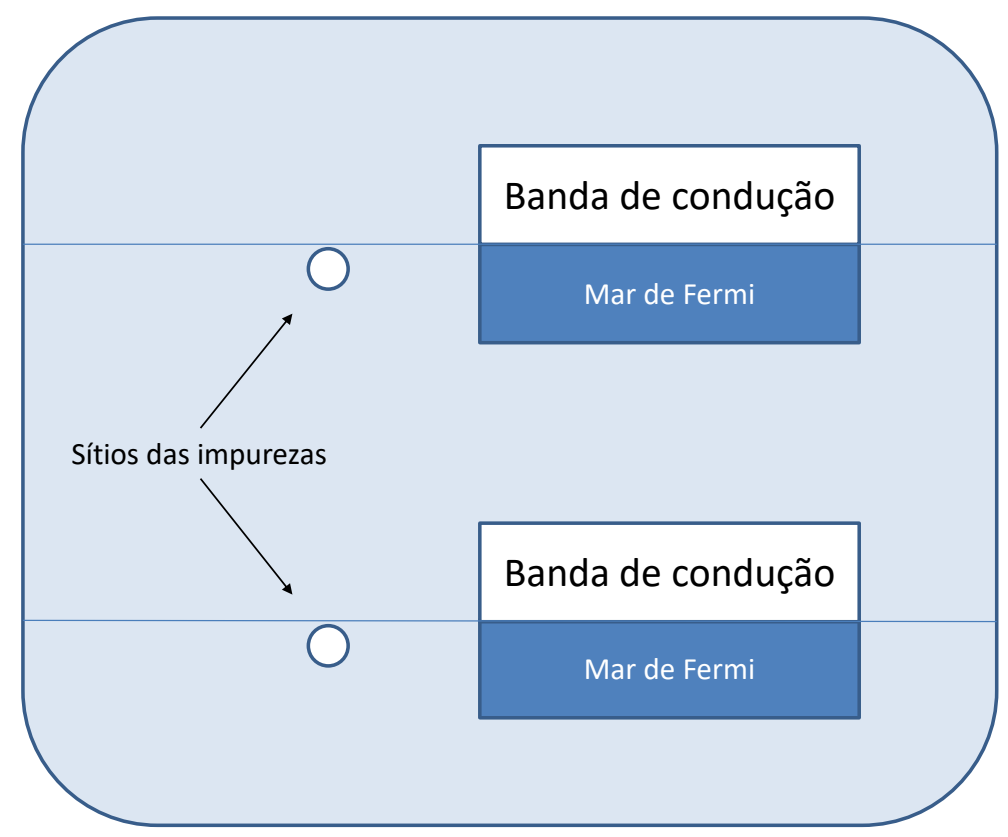

Figura A.5: Representação diagramática de escolha do subespaço $S_{1}$ para o sistema com duas impurezas. O estado escolhido para constituir este subespaço possui as duas impurezas vazias e os mares de Fermi no estado fundamental.

No sistema de duas impurezas, os operadores que conectam os subespaços $S_{1}$ e $S_{2}$ são rigorosamente os mesmos que no caso de apenas uma impureza, equações (A-14) e (A-15).

A família de estados I, originária de aplicar o operador $H_{21}$ sobre $\psi_{1}$, é formada pelo estado com uma impureza populada e um buraco com momento $k$ no mar de Fermi correspondente e a outra impureza vazia, figura A.6. Como as repulsões Coulombianas tanto intraponto como interponto são infinitas, desta família de estados podemos apenas ir à família de estados II com as impurezas 
vazias e o elétron, que ocupava uma impureza na família anterior, enviado à banda de condução, conforme ilustrado nos diagramas da figura A.6. Assim como no modelo de Anderson com apenas uma impureza, a família de estados III acessada é análoga a família de estados I, a menos de um segundo buraco no nível de Fermi com elemento diagonal $\epsilon_{\boldsymbol{k}^{\prime}}$, ver diagrama da figura A.6.

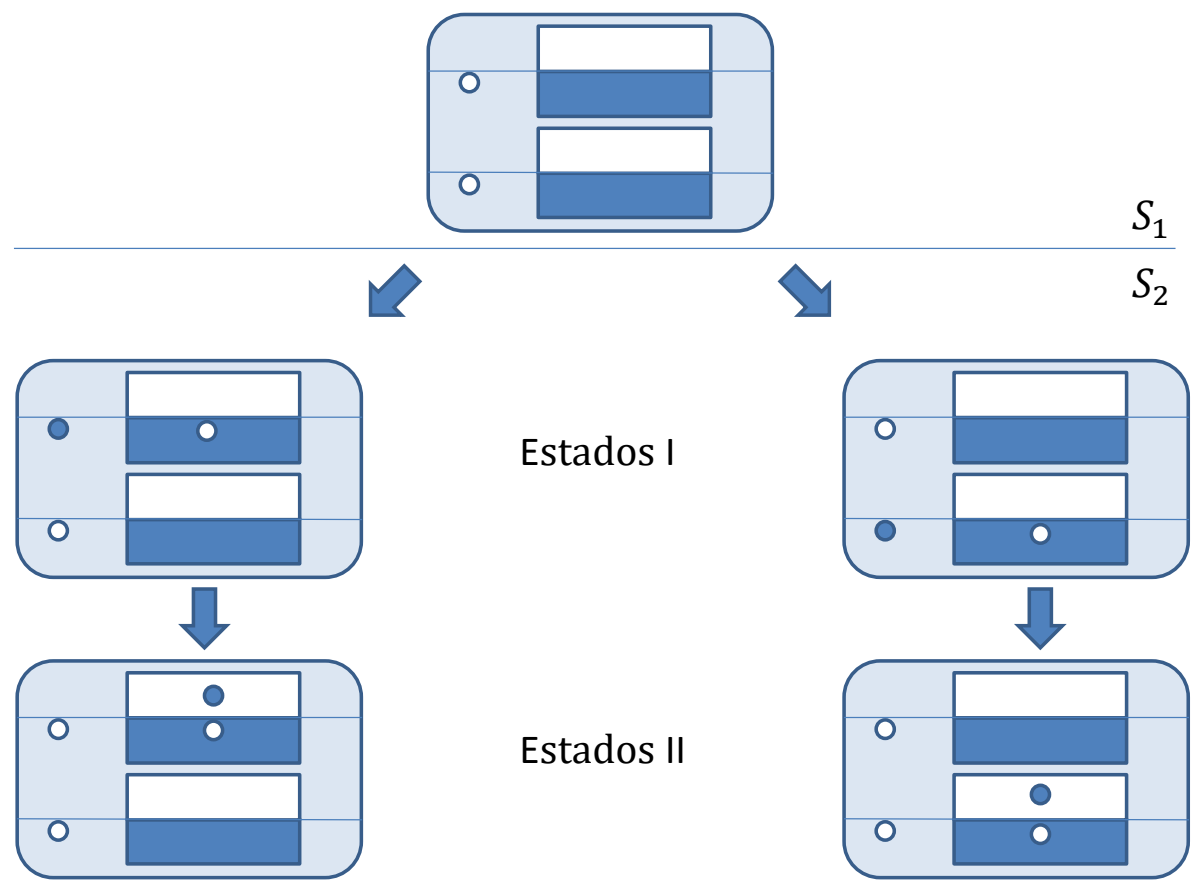

Figura A.6: Diagrama apresentando a construção do subespaço $S_{2}$, com a mesma notação da figura A.5. Na parte superior da figura, temos a única família de estados que constitui o subespaço $S_{1}$. As setas abaixo deste estado mostram a passagem do subespaço $S_{1}$ a primeira família de estados do subespaço $S_{2}$, estados I, com a impureza ocupada e um buraco no mar de Fermi correspondente a esta impureza. Também ilustramos a passagem aos estados II, onde o elétron da impureza dirige-se à banda de condução.

Supondo que os dois subsistemas - caracterizado pelo conjunto do PQ, mar de Fermi, banda de condução e os contatos entre a impureza e mar de Fermi e banda de condução - sejam iguais, a diferença em relação ao cálculo de apenas uma impureza resulta em um fator numérico 2 , referente justamente à cada subsistema, o qual é independente do outro.

Logo, o cálculo da correção à energia do estado fundamental apresenta a forma

$$
\begin{array}{r}
\Delta E=\frac{1}{N} \sum_{\epsilon_{\boldsymbol{k} \sigma}<\epsilon_{F}} \frac{2 V^{2}}{\Delta E+\epsilon_{\boldsymbol{k} \sigma}-\epsilon_{0 \sigma}-\frac{1}{N} \sum_{\epsilon_{\boldsymbol{K} \sigma}>\epsilon_{F}} \frac{V^{2}}{\Delta E+\epsilon_{\boldsymbol{k} \sigma}-\epsilon_{\boldsymbol{K} \sigma}-\Sigma}} \\
\Sigma=\frac{1}{N} \sum_{\epsilon_{\boldsymbol{k}^{\prime} \sigma}<\epsilon_{F}} \frac{2 V^{2}}{\Delta E+\epsilon_{\boldsymbol{k} \sigma}-\epsilon_{\boldsymbol{K} \sigma}+\epsilon_{\boldsymbol{k}^{\prime} \sigma}-\epsilon_{0 \sigma}-\ldots}
\end{array}
$$


No caso de duas impurezas é conveniente definir a correção a energia do estado fundamental, $\Delta E$, como

$$
\Delta E \equiv E-2 \epsilon_{T}
$$

onde $\epsilon_{T}$ é, para cada mar de Fermi, o somatório das energias dos elétrons por baixo do nível de Fermi, dada por

$$
\epsilon_{T}=2 \int_{-\infty}^{\epsilon_{F}} \omega \rho(\omega) d \omega
$$

e $\rho(\omega)$ é a densidade de estados do mar de Fermi de cada contato. O fator 2 na definição de $\Delta E$, equação (A-50), deve-se a presença dos dois mares de Fermi, enquanto que na expressão de $\epsilon_{T}$ é oriundo a degenerescência de spin.

Da mesma forma que no caso com apenas uma impureza, a correção à energia do estado fundamental $\Delta E$ é encontrada através de

$$
\Delta E=f_{1}(\Delta E),
$$

onde as funções $f_{1}(\xi)$ e $f_{0}(\xi)$, no limite termodinâmico, são dadas por

$$
f_{1}(\xi)=\sum_{\sigma} \int_{-\infty}^{\epsilon_{F}}\left\{\rho(\omega) \frac{2 V^{2}}{\xi+\omega-\epsilon_{0}+\sigma B-f_{0}(\xi+\omega)}\right\} d \omega
$$

$\mathrm{e}$

$$
f_{0}(\xi)=\int_{\epsilon_{F}}^{\infty}\left\{\rho(\omega) \frac{V^{2}}{\xi-\omega-f_{1}(\xi-\omega)}\right\} d \omega
$$

e devem ser resolvidas autoconsistentemente.

\section{A.6 \\ Resumo do apêndice}

Foi apresentado neste apêndice um resumo do formalismo dos operadores de projeção, o qual permite obter a energia do estado fundamental do Hamiltoniano da impureza magnética de Anderson. Ele consiste em fazer uma projeção do espaço de Hilbert em dois subespaços que permitem obter a solução do problema num espaço unidimensional. Ao projetar o Hamiltoniano no subespaço $S_{1}$ obtemos um Hamiltoniano renormalizado com o mesmo autovalor da equação de Schrödinger original (A-8). Através de um cálculo autoconsistente de duas funções, $f_{1 \sigma}(\xi)$ e $f_{0 \sigma}(\xi)$, equações (A-28) e (A-29), mostramos como obter a energia do estado fundamental do sistema. Usando o formalismo das funções de Green apresentamos a equivalência do problema de uma impureza ao de uma rede de Bethe com número de coordenação infinito, corroborando as expressões dos termos diagonais e permitindo o cálculo dos termos não diagonais, correção de ordem superior em potências de $V$. Finalmente, na seção A.5 estendemos o formalismo para tratar sistemas com duas impurezas idênticas. 
B

\section{Cálculo da condutância}

Neste apêndice é apresentado os cálculos que relacionam a condutância com o número de ocupação no ponto quântico (PQ).

A função de Green para um PQ conectado a um reservatório eletrônico pode ser escrita como

$$
G_{00}^{\sigma \sigma}(\omega)=\frac{1}{\omega-\epsilon_{0}-\Sigma_{1 c}(\omega)-\Sigma_{m c}(\omega)-i \eta},
$$

onde $\Sigma_{1 c}(\omega)$ e $\Sigma_{m c}(\omega)$ são as autoenergias de um e de muitos corpos, respectivamente, e $\eta$ é um pequeno deslocamento no plano complexo para regularizar as funções de Green no eixo real.

Ao calcularmos a derivada do logaritmo de $G_{00}^{\sigma \sigma}(\omega)$ obtemos a identidade

$$
\begin{aligned}
\frac{\partial}{\partial \omega} \ln \left[G_{00}^{\sigma \sigma}(\omega)\right]^{-1} & =\frac{\partial}{\partial \omega} \ln \left[\omega-\epsilon_{0}-\Sigma_{1 c}(\omega)-\Sigma_{m c}(\omega)\right] \\
& =G_{00}^{\sigma \sigma}(\omega)\left[1-\frac{\partial}{\partial \omega} \Sigma_{1 c}(\omega)-\frac{\partial}{\partial \omega} \Sigma_{m c}(\omega)\right]
\end{aligned}
$$

que pode ser reescrita como

$$
G_{00}^{\sigma \sigma}(\omega)=\frac{\partial}{\partial \omega} \ln \left[G_{00}^{\sigma \sigma}(\omega)\right]^{-1}+G_{00}^{\sigma \sigma}(\omega)\left[\frac{\partial}{\partial \omega} \Sigma_{1 c}(\omega)+\frac{\partial}{\partial \omega} \Sigma_{m c}(\omega)\right] .
$$

Multiplicando ambos os lado por $1 / \pi$, tomando a parte imaginária e integrando de $\omega \rightarrow-\infty$ ao nível de Fermi, $\epsilon_{F}$, obtemos:

$$
\begin{gathered}
\frac{1}{\pi} \int_{-\infty}^{\epsilon_{F}} \Im G_{00}^{\sigma \sigma}(\omega) d \omega=\frac{1}{\pi} \int_{-\infty}^{\epsilon_{F}} \Im\left\{\frac{\partial}{\partial \omega} \ln \left[G_{00}^{\sigma \sigma}(\omega)\right]^{-1}\right\} d \omega+ \\
\frac{1}{\pi} \int_{-\infty}^{\epsilon_{F}} \Im\left\{G_{00}^{\sigma \sigma}(\omega)\left[\frac{\partial}{\partial \omega} \Sigma_{1 c}(\omega)+\frac{\partial}{\partial \omega} \Sigma_{m c}(\omega)\right]\right\} d \omega .
\end{gathered}
$$

Usando a definição do número de ocupação,

$$
\left\langle n_{\sigma}\right\rangle=\frac{1}{\pi} \int_{-\infty}^{\epsilon_{F}} \Im G_{00}^{\sigma \sigma}(\omega) d \omega,
$$

e a seguinte propriedade do líquido de Fermi [7]

$$
\Im \int_{-\infty}^{\epsilon_{F}} \frac{\partial}{\partial \omega} \Sigma_{m c}(\omega) G_{00}^{\sigma \sigma}(\omega) d \omega=0,
$$

obtemos a partir da equação (B-4) que, 


$$
\left\langle n_{\sigma}\right\rangle=\frac{1}{\pi} \Im\left\{\ln \left[G_{00}^{\sigma \sigma}(\omega)\right]^{-1}\right\}_{-\infty}^{\epsilon_{F}}+\frac{1}{\pi} \int_{-\infty}^{\epsilon_{F}} \Im G_{00}^{\sigma \sigma}(\omega) \frac{\partial}{\partial \omega} \Sigma_{1 c}(\omega) d \omega .
$$

Por outro lado, podemos escrever a função de Green em notação trigonométrica de um número complexo como

$$
G_{00}^{\sigma \sigma}(\omega)=\left|G_{00}^{\sigma \sigma}(\omega)\right| e^{i \theta(\omega)},
$$

onde $\theta(\omega)$ é o ângulo entre $\Re G_{00}^{\sigma \sigma}(\omega)$ e $\left|G_{00}^{\sigma \sigma}(\omega)\right|$, como ilustrado na figura B.1.

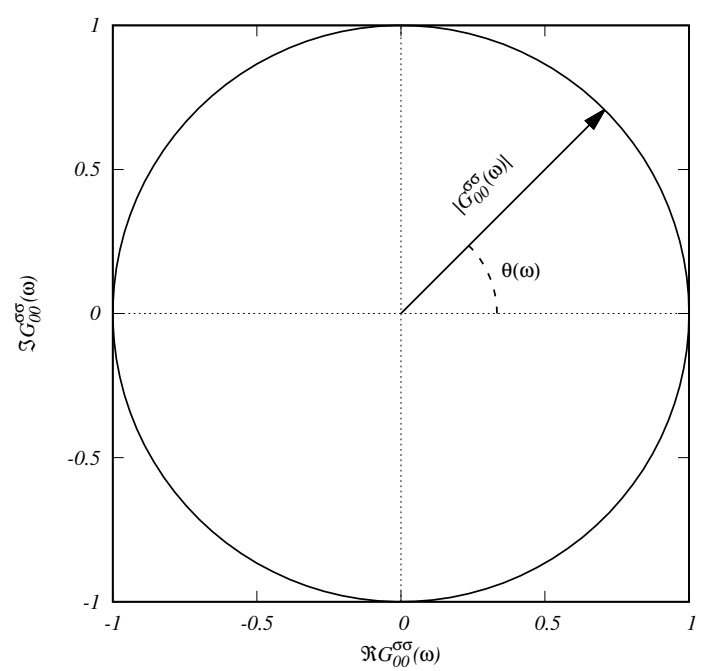

Figura B.1: Representação trigonométrica da função de Green $G_{00}^{\sigma \sigma}(\omega)$, equação (B-8).

Assim, o primeiro termo do lado direito da equação (B-7) poderá ser relacionado $\operatorname{com} \theta(\omega)$ por

$$
\Im \ln \left[G_{00}^{\sigma \sigma}(\omega)\right]^{-1}=-\theta(\omega) .
$$

Substituindo este último resultado na equação (B-7), podemos reescrevêla como

$$
\pi\left(\left\langle n_{\sigma}\right\rangle-I\right)=\left(\theta\left(\epsilon_{F}\right)-\theta(-\infty)\right),
$$

onde definimos a integral $I$ como

$$
I=\frac{1}{\pi} \int_{-\infty}^{\epsilon_{F}} \Im G_{00}^{\sigma \sigma}(\omega) \frac{\partial}{\partial \omega} \Sigma_{1 c}(\omega) d \omega .
$$

Ainda resta calcular $\theta(\omega)$ em $\omega=\epsilon_{F}$ e no limite de $\omega \rightarrow-\infty$. Primeiramente iremos analisar o limite quando $\omega \rightarrow-\infty$ a partir do comportamento assintótico bem conhecido das funções de Green,

$$
\lim _{|\omega| \rightarrow \infty} G_{00}^{\sigma \sigma}(\omega)=\frac{1}{\omega} .
$$


Com base no comportamento assintótico descrito na equação anterior, vemos que a parte imaginária de $G_{00}^{\sigma \sigma}(-\infty)$ é zero, o que resulta em $\theta(-\infty)=\pi$. Substituindo este resultado na equação (B-10), temos

$$
\theta\left(\epsilon_{F}\right)=\pi\left(I-\left\langle n_{\sigma}\right\rangle\right)+\pi
$$

Para calcular $\theta\left(\epsilon_{F}\right)$, partiremos da relação no plano complexo da parte imaginária e do módulo de $\theta(\omega)$, figura B.1, conseguindo

$$
\operatorname{sen}[\theta(\omega)]=\frac{\Im G_{00}^{\sigma \sigma}(\omega)}{\left|G_{00}^{\sigma \sigma}(\omega)\right|} .
$$

Contudo, antes de prosseguir precisamos de outra relação entre o módulo e a parte imaginária de $G_{00}^{\sigma \sigma}(\omega)$, a qual pode ser obtida a partir da equação (B-1).

Ao separarmos as contribuições das partes reais e imaginárias das autoenergias de um corpo e de muitos corpos, respectivamente $\Sigma_{1 c}(\omega)$ e $\Sigma_{m c}(\omega)$, escrevermos a função de Green $G_{00}^{\sigma \sigma}(\omega)$ como,

$$
G_{00}^{\sigma \sigma}(\omega)=\frac{1}{\omega-\epsilon_{0}-\Re \Sigma_{1 c}(\omega)-\Re \Sigma_{m c}(\omega)-i\left[\Im \Sigma_{1 c}(\omega)+\Im \Sigma_{m c}(\omega)\right]}
$$

e seu módulo quadrado como,

$$
\left|G_{00}^{\sigma \sigma}(\omega)\right|^{2}=\frac{1}{\left[\omega-\epsilon_{0}-\Re \Sigma_{1 c}(\omega)-\Re \Sigma_{m c}(\omega)\right]^{2}+\left[\Im \Sigma_{1 c}(\omega)+\Im \Sigma_{m c}(\omega)\right]^{2}} .
$$

Multiplicando ambos, o numerador e o denominador da equação (B-15), pelo conjugado complexo de seu denominador e tomando sua parte imaginária, temos que,

$$
\Im G_{00}^{\sigma \sigma}(\omega)=\left|G_{00}^{\sigma \sigma}(\omega)\right|^{2} \Im\left[\Sigma_{1 c}(\omega)+\Sigma_{m c}(\omega)\right] .
$$

Cujo valor ao nível de Fermi é

$$
\Im G_{00}^{\sigma \sigma}\left(\epsilon_{F}\right)=\left|G_{00}^{\sigma \sigma}\left(\epsilon_{F}\right)\right|^{2} \Im \Sigma_{1 c}\left(\epsilon_{F}\right)
$$

onde usamos outra propriedade do líquido de Fermi [7],

$$
\Im \Sigma_{m c}\left(\epsilon_{F}\right)=0 \text {. }
$$

Elevando ao quadrado ambos os lados da equação (B-14), calculada ao nível de Fermi, e usando a equação (B-18) temos que

$$
\operatorname{sen}^{2} \theta\left(\epsilon_{F}\right)=\left|G_{00}^{\sigma \sigma}\left(\epsilon_{F}\right)\right|^{2}\left[\Im \Sigma_{1 c}\left(\epsilon_{F}\right)\right]^{2} \text {. }
$$

Calculamos o sen $\left[\theta\left(\epsilon_{F}\right)\right]$ usando a equação (B-13), uma identidade trigonométrica e a propriedade de paridade ímpar da função seno, obtendo

$$
\operatorname{sen}^{2}\left[\theta\left(\epsilon_{F}\right)\right]=\operatorname{sen}^{2}\left[\pi\left(\left\langle n_{\sigma}\right\rangle-I\right)\right] .
$$

Finalmente, conseguimos relacionar $\left|G_{00}^{\sigma \sigma}\left(\epsilon_{F}\right)\right|^{2} \operatorname{com}\left\langle n_{\sigma}\right\rangle$, através das 
equações (B-20) e (B-21), resultando em,

$$
\left|G_{00}^{\sigma \sigma}\left(\epsilon_{F}\right)\right|^{2}=\frac{\operatorname{sen}^{2}\left[\pi\left(\left\langle n_{\sigma}\right\rangle-I\right)\right]}{\Delta^{2}},
$$

onde definimos a função de hibridização como

$$
\Delta=\pi V^{2} \rho\left(\epsilon_{F}\right)=\Im \Sigma_{1 c}\left(\epsilon_{F}\right)
$$

sendo $\rho\left(\epsilon_{F}\right)$ a densidade de estados ao nível de Fermi.

Por fim, obtemos uma expressão para calcular a condutância a partir dos números de ocupação nos $\mathrm{PQ}$, dada por

$$
C^{\sigma}\left(\frac{e}{h}\right)=4 \pi^{2} V_{e}^{4} \rho_{-1}\left(\epsilon_{F}\right) \rho_{1}\left(\epsilon_{F}\right) \frac{\operatorname{sen}^{2}\left[\pi\left(\left\langle n_{\sigma}\right\rangle-I\right)\right]}{\Delta^{2}} .
$$

No cálculo que segue, o valor da integral $I$ será desprezado, pois estamos assumindo uma banda de condução plana para descrever a densidade de estados.

A expressão para a condutância depende fortemente da geometria do sistema. Inicialmente analisaremos o caso do PQ embebido localizado no sítio 0, o qual está conectado a duas semi-cadeias lineares infinitas que começam nos sítios -1 e 1 . Para esta geometria, os elementos de matriz não diagonais que conectam o $\mathrm{PQ}$ aos contatos de ambas as semicadeias são $V_{e}=V$ e a densidade de estados de uma semicadeia linear apresenta a forma

$$
\rho=\frac{\sqrt{4 t^{2}-\omega^{2}}}{2 \pi t^{2}} .
$$

A função de hibridização calculada no nível de Fermi para esta configuração resulta ser,

$$
\Delta\left(\epsilon_{F}\right)=\frac{2 V^{2}}{t}
$$

Consequentemente, a expressão para a condutância do PQ embebido é

$$
C^{\sigma}\left(\frac{e^{2}}{h}\right)=\operatorname{sen}^{2}\left(\frac{\pi}{2}\left\langle n_{\sigma}\right\rangle\right)
$$

onde $\left\langle n_{\sigma}\right\rangle$ é a ocupação em ambos os PQs, conforme definido no capítulo 2 do texto principal, por isso do fator $1 / 2$ no argumento do seno.

Para a geometria dos PQs conectados lateralmente, é necessário relacionar a função de Green nos PQ, que chamaremos de $G_{d d}^{\sigma \sigma}(\omega)$, com a função de Green no sítio $i=0, G_{00}^{\sigma \sigma}(\omega)$, e a função de Green despida de uma cadeia linear infinita, $g_{0}$ (figura 2.1 (b)). Esta relação ocorre através da equação (2-13), que repetiremos aqui,

$$
G_{00}^{\sigma \sigma}\left(\epsilon_{F}\right)=g_{0}+g_{0}^{2} V^{2} G_{d d}^{\sigma \sigma}\left(\epsilon_{F}\right)
$$

Nesta geometria, os elementos de matriz não diagonais dos contatos entre os sítios $i=0$ e $i= \pm 1$ são $t$, i.e. $V_{e}=t$, como podem ser vistos na figura 2.1. A 
densidade de estados neste caso é

$$
\rho(\omega)=\frac{1}{\pi \sqrt{4 t^{2}-\omega^{2}}}
$$

e a função de hibridização é

$$
\Delta=\frac{V^{2}}{2 t}
$$

Logo, obtemos a equação que relaciona os módulos de $G_{d d}^{\sigma \sigma}(\omega)$ com $G_{00}^{\sigma \sigma}(\omega)$,

$$
\left|G_{00}^{\sigma \sigma}(\omega)\right|^{2}=\frac{1}{4 t^{2}}-\frac{V^{4}}{16 t^{4}}\left|G_{d d}^{\sigma \sigma}\left(\epsilon_{F}\right)\right|^{2},
$$

Usando esta última equação e com um raciocínio análogo ao empregado para o PQ embebido, a condutância para o PQ conectado lateralmente resulta ser,

$$
C^{\sigma}\left(\frac{e^{2}}{h}\right)=1-\operatorname{sen}^{2}\left(\frac{\pi}{2}\left\langle n_{\sigma}\right\rangle\right)
$$

As equações (B-27) e (B-32) são as utilizadas (equações (2-14) e (2-15)) no texto principal para o cálculo da condutância do sistema. 


\section{C \\ Transformação do Hamiltoniano com interação spin-órbita}

Neste apêndice, mostraremos os cálculos detalhados da transformação do Hamiltoniano de uma cadeia linear infinita com interação SO da base dos spins $\sigma$ para a base da helicidade $\nu$, onde ele é diagonal. Esta mudança de base é realizada pela transformação definida na equação (3-20) do texto principal, que repetimos aqui,

$$
c_{k \nu}^{\dagger}=\frac{1}{\sqrt{2}}\left(\nu e^{i \phi} \operatorname{sinal}[\operatorname{sen}(k a)] c_{k \uparrow}^{\dagger}+c_{k \downarrow}^{\dagger}\right) .
$$

Explicitamente, para cada valor de $\nu= \pm$ temos,

$$
\begin{gathered}
c_{k+}^{\dagger}=\frac{1}{\sqrt{2}}\left(e^{i \phi} \operatorname{sinal}[\operatorname{sen}(k a)] c_{k \uparrow}^{\dagger}+c_{k \downarrow}^{\dagger}\right) \\
c_{k-}^{\dagger}=\frac{1}{\sqrt{2}}\left(-e^{i \phi} \operatorname{sinal}[\operatorname{sen}(k a)] c_{k \uparrow}^{\dagger}+c_{k \downarrow}^{\dagger}\right) .
\end{gathered}
$$

A transformação inversa é obtida subtraindo e somando as equações (C-2) e (C-3), obtendo,

$$
c_{k \uparrow}^{\dagger}=\frac{1}{\sqrt{2}} e^{-i \phi} \sin a l[\operatorname{sen}(k a)]\left(c_{k+}^{\dagger}-c_{k-}^{\dagger}\right)
$$

e

$$
c_{k \downarrow}^{\dagger}=\frac{1}{\sqrt{2}}\left(c_{k+}^{\dagger}+c_{k-}^{\dagger}\right) .
$$

O operador de aniquilação é obtido tomando o hermitiano conjugado das equações anteriores, equações (C-1), (C-2) e (C-3) temos, respectivamente,

$$
c_{k \nu}=\frac{1}{\sqrt{2}}\left(\nu e^{-i \phi} \sin a l[\operatorname{sen}(k a)] c_{k \uparrow}+c_{k \downarrow}\right),
$$

Explicitamente, para cada valor de $\nu= \pm$ temos,

$$
c_{k+}=\frac{1}{\sqrt{2}}\left(e^{-i \phi} \operatorname{sinal}[\operatorname{sen}(k a)] c_{k \uparrow}+c_{k \downarrow}\right)
$$

$\mathrm{e}$

$$
c_{k-}=\frac{1}{\sqrt{2}}\left(-e^{-i \phi} \operatorname{sinal}[\operatorname{sen}(k a)] c_{k \uparrow}+c_{k \downarrow}\right) .
$$

Analogamente para a transformação inversa, equações (C-4) e (C-5)

$$
c_{k \uparrow}=\frac{1}{\sqrt{2}} e^{i \phi} \operatorname{sinal}[\operatorname{sen}(k a)]\left(c_{k+}-c_{k-}\right)
$$

e

$$
c_{k \downarrow}=\frac{1}{\sqrt{2}}\left(c_{k+}+c_{k-}\right) .
$$


O Hamiltoniano de uma cadeia linear infinita possui a forma

$$
H=-\sum_{k, \sigma} 2 t \cos (k a) c_{k \sigma}^{\dagger} c_{k \sigma}-\sum_{k} 2 \operatorname{sen}(k a)\left(\gamma c_{k \uparrow}^{\dagger} c_{k \downarrow}+\gamma^{*} c_{k \downarrow}^{\dagger} c_{k \uparrow}\right),
$$

conforme mostramos no texto principal desta tese, equação (3-16).

Os termos diagonais em spin $\sigma$ são reescritos na nova base da helicidade $\nu$ usando as transformações apresentadas, resultando em

$$
c_{k \uparrow}^{\dagger} c_{k \uparrow}=\frac{1}{2}\{\operatorname{sinal}[\operatorname{sen}(k a)]\}^{2}\left(c_{k+}^{\dagger} c_{k+}-c_{k+}^{\dagger} c_{k-}-c_{k-}^{\dagger} c_{k+}-c_{k-}^{\dagger} c_{k-}\right)
$$

$\mathrm{e}$

$$
c_{k \downarrow}^{\dagger} c_{k \downarrow}=\frac{1}{2}\left(c_{k+}^{\dagger} c_{k+}+c_{k+}^{\dagger} c_{k-}+c_{k-}^{\dagger} c_{k+}+c_{k-}^{\dagger} c_{k-}\right) .
$$

Ao somarmos estas duas equações os termos não diagonais em $\nu$ cancelamse e só restam os diagonais. Assim, o termo diagonal em $\sigma$ do Hamiltoniano permanece diagonal na base $\nu$,

$$
-\sum_{k, \sigma} 2 t \cos (k a) c_{k \sigma}^{\dagger} c_{k \sigma}=-\sum_{k, \nu} 2 t \cos (k a) c_{k \nu}^{\dagger} c_{k \nu} .
$$

Os termos não diagonais em $\sigma$ ficam, nesta nova base,

$$
\gamma c_{k \uparrow}^{\dagger} c_{k \downarrow}=\frac{\gamma}{2} e^{-i \phi} \operatorname{sinal}[\operatorname{sen}(k a)]\left(c_{k+}^{\dagger} c_{k+}+c_{k+}^{\dagger} c_{k-}-c_{k-}^{\dagger} c_{k+}-c_{k-}^{\dagger} c_{k-}\right)
$$

e

$$
\gamma^{*} c_{k \downarrow}^{\dagger} c_{k \uparrow}=\frac{\gamma^{*}}{2} e^{i \phi} \operatorname{sinal}[\operatorname{sen}(k a)]\left(c_{k+}^{\dagger} c_{k+}-c_{k+}^{\dagger} c_{k-}+c_{k-}^{\dagger} c_{k+}-c_{k-}^{\dagger} c_{k-}\right) .
$$

A soma destes dois termos resulta ser:

$$
\begin{aligned}
\gamma c_{k \uparrow}^{\dagger} c_{k \downarrow}+\gamma^{*} c_{k \downarrow}^{\dagger} c_{k \uparrow}=\frac{1}{2} \operatorname{sinal}[\operatorname{sen}(k a)] \times & \left\{\left(\gamma e^{-i \phi}+\gamma^{*} e^{i \phi}\right) c_{k+}^{\dagger} c_{k+}+\left(\gamma e^{-i \phi}-\gamma^{*} e^{i \phi}\right) c_{k+}^{\dagger} c_{k-}+\right. \\
& \left.\left(-\gamma e^{-i \phi}+\gamma^{*} e^{i \phi}\right) c_{k-}^{\dagger} c_{k+}-\left(\gamma e^{-i \phi}+\gamma^{*} e^{i \phi}\right) c_{k-}^{\dagger} c_{k-}\right\}
\end{aligned}
$$

Da definição de $\phi$,

$$
\phi=\operatorname{tg}^{-1}\left(\frac{\alpha}{\beta}\right)
$$

temos que,

$$
\operatorname{sen} \phi=\frac{\alpha}{|\gamma|} \text { e } \cos \phi=\frac{\beta}{|\gamma|}
$$

O que permite escrever

$$
e^{-i \phi}=\frac{\gamma^{*}}{|\gamma|} \text { e } e^{i \phi}=\frac{\gamma}{|\gamma|}
$$

Substituindo esses resultados na equação (C-17) só sobrevivem os termos diagonais em $\nu$. Após alguma álgebra, o termo não diagonal em $\sigma$ resulta em, 


$$
-\sum_{k} 2 \operatorname{sen}(k a)\left(\gamma c_{k \uparrow}^{\dagger} c_{k \downarrow}+\gamma^{*} c_{k \downarrow}^{\dagger} c_{k \uparrow}\right)=-\sum_{k, \nu} 2 \nu|\gamma||\operatorname{sen}(k a)| c_{k \nu}^{\dagger} c_{k \nu}
$$

Finalmente, o Hamiltoniano de uma cadeia linear infinita com interação SO fica

$$
H=\sum_{k, \nu} \epsilon_{k \nu} c_{k \nu}^{\dagger} c_{k \nu}
$$

onde definimos

$$
\epsilon_{k \nu}=-2 t \cos (k a)-2 \nu|\gamma||\operatorname{sen}(k a)| \text {. }
$$

